\title{
Relativistic Quantum Chaos
}

Liang Huang, ${ }^{1}$ Hong-Ya Xu, ${ }^{2}$ Celso Grebogi, ${ }^{3,4}$ and Ying-Cheng Lai ${ }^{2,5, *}$

${ }^{1}$ School of Physical Science and Technology,

and Key Laboratory for Magnetism and Magnetic Materials of MOE,

Lanzhou University, Lanzhou, Gansu 730000, China

${ }^{2}$ School of Electrical, Computer, and Energy Engineering,

Arizona State University, Tempe, AZ 85287, USA

${ }^{3}$ Institute for Complex Systems and Mathematical Biology,

School of Natural and Computing Sciences, King's College,

University of Aberdeen, Aberdeen AB24 3UE, UK

${ }^{4}$ Shaanxi Key Laboratory of Complex System Control and Intelligent Information Processing,

$X i$ 'an University of Technology, Xi'an 710048, China

${ }^{5}$ Department of Physics, Arizona State University, Tempe, AZ 85287, USA

(Dated: March 28, 2018) 


\begin{abstract}
Quantum chaos is generally referred to as the study of quantum manifestations or fingerprints of nonlinear dynamical and chaotic behaviors in the corresponding classical system, an interdisciplinary field that has been active for about four decades. In closed chaotic Hamiltonian systems, for example, the basic phenomena studied include energy level-spacing statistics and quantum scarring. In open Hamiltonian systems, quantum chaotic scattering has been investigated extensively. Previous works were almost exclusively for nonrelativistic quantum systems described by the Schrödinger equation. Recent years have witnessed a rapid growth of interest in Dirac materials such as graphene, topological insulators, molybdenum disulfide and topological Dirac semimetals. A common feature of these materials is that their physics is described by the Dirac equation in relativistic quantum mechanics, generating phenomena that do not usually emerge in conventional semiconductor materials. This has important consequences. In particular, at the level of basic science, a new field has emerged: Relativistic Quantum Chaos (RQC), which aims to uncover, understand, and exploit relativistic quantum manifestations of classical nonlinear dynamical behaviors including chaos. Practically, Dirac materials have the potential to revolutionize solid-state electronic and spintronic devices, and have led to novel device concepts such as valleytronics. Exploiting manifestations of nonlinear dynamics and chaos in the relativistic quantum regime can have significant applications.

The aim of this article is to give a comprehensive review of the basic results obtained so far in the emergent field of RQC. Phenomena to be discussed in depth include energy level-spacing statistics in graphene or Dirac fermion systems that exhibit various nonlinear dynamical behaviors in the classical limit, relativistic quantum scars (unusually high concentrations of relativistic quantum spinors about classical periodic orbits), peculiar features of relativistic quantum chaotic scattering and quantum transport, manifestations of the Klein paradox and its effects on graphene or 2D Dirac material based devices, chaos based modulation of conductance fluctuations in relativistic quantum dots, regularization of relativistic quantum tunneling by chaos, superpersistent currents in chaotic Dirac rings subject to a magnetic flux, and exploitation of relativistic quantum whispering gallery modes for applications in quantum information science. Computational methods for solving the Dirac equation in various situations will be introduced and physical theories developed so far in RQC will be described. Potential device applications will be discussed.
\end{abstract}

\footnotetext{
*Ying-Cheng.Lai@asu.edu
} 


\section{CONTENTS}

$\begin{array}{ll}\text { I. Introduction } & 6\end{array}$

A. Background: the traditional field of quantum chaos $\quad 6$

B. Relativistic quantum chaos: why, what, and how?

1. Why is it important to study RQC?

2. What has been done in RQC?

3. How was previous research done in RQC?

C. Outline of this Review

II. Relativistic quantum billiards and solution methods 12

A. Graphene billiard $\quad 12$

1. Tight-binding Hamiltonian 12

2. Boundary conditions for graphene billiards $\quad 13$

3. Interacting electrons in graphene billiard 14

B. Microwave billiards with a triangular photonic structure $\quad 15$

$\begin{array}{ll}\text { C. Dirac billiard } & 17\end{array}$

1. Dirac equation in a closed domain $\quad 17$

2. Boundary conditions for Dirac billiards $\quad 19$

D. Finite element method for solving the Dirac equation in two dimensions 20

1. Discretization scheme and elimination of fermion doubling effect 20

2. Boundary conditions and matrix representation of Dirac equation 21

3. Validation of solution method using a ring domain for which the Dirac equation is $\begin{array}{ll}\text { analytically solvable } & 22\end{array}$

E. Boundary integral method for solving the Dirac equation in two dimensions 23

F. Conformal mapping method for solving the Dirac equation in two-dimensional $\begin{array}{ll}\text { chaotic billiards } & 27\end{array}$

1. Analytic solution of the Dirac equation in the circular domain 27

2. Conformal mapping $\quad 29$

G. Solutions of the Dirac equation in a ring domain subject to a central magnetic flux $\quad 30$

1. Effects of a magnetic flux on the energy spectra 30

2. Dirac billiard with a singular Aharonov-Bohm (AB) magnetic flux 32

III. Energy level-spacing statistics in relativistic quantum chaotic systems 41

A. Energy level-spacing statistics - an introduction $\quad 41$

B. Characterization of level-spacing statistics $\quad 42$

C. Dirac billiards - GUE statistics without a magnetic field 45

D. Graphene billiards $\quad 46$

1. Basics $\quad 46$

2. Effects of disorders and edge roughness 46

3. Effects of chaos on level-spacing statistics in graphene systems 49

4. Level spacing statistics for edge states in chaotic graphene billiards 53

5. Can valley decoupling lead to GUE statistics in graphene billiards without any magnetic field? 54

6. Peculiar level-spacing statistics in circular sector graphene billiards 57 
E. Microwave Dirac billiards $\quad 60$

F. Concluding remarks on energy level-spacing statistics 62

$\begin{array}{ll}\text { IV. Relativistic quantum scars } & 63\end{array}$

$\begin{array}{ll}\text { A. Quantum scars - an introduction } & 63\end{array}$

$\begin{array}{ll}\text { B. Quantum scars in graphene billiards } & 64\end{array}$

C. Chiral scars in chaotic Dirac billiards $\quad 68$

D. Fundamental mechanism of chiral scars and their control via an external magnetic field $\quad 72$

$\begin{array}{ll}\text { 1. Symmetry considerations } & 72\end{array}$

2. Time-reversal symmetry breaking and chiral scars $\quad 73$

3. Change in scar chirality due to a magnetic flux $\quad 77$

4. Semiclassical formula of chiral scars $\quad 79$

5. Control of chirality of scars via an external magnetic field 79

E. Effects of symmetry operations on spin and chiral scars 82

1. Negative energy $(-E)$ and positive potential $(V)$ with $V>E>0 \quad 82$

2. Positive energy $(E)$, negative potential $(-V)$ and $V>E>0 \quad 84$

3. Negative energy $(-E)$, negative potential $(-V)$ and $V>E>0 \quad 86$

4. Mirror reflection symmetry $\quad 88$

5. Parity operation $\quad 89$

6. Summary of spin characteristics of chaotic Dirac billiard under combinations of $\begin{array}{ll}\text { three basic symmetry operations } & 90\end{array}$

F. Outlook and remarks on relativistic quantum scars 90

V. Relativistic quantum chaotic scattering $\quad 93$

A. Quantum chaotic scattering - an introduction 93

B. Conductance fluctuations associated with quantum transport and the relevance of $\begin{array}{ll}\text { quantum chaotic scattering } & 94\end{array}$

C. Relativistic quantum chaotic scattering in graphene quantum dots 95

D. Quantum chaotic scattering for bilayer graphene quantum dots 102

E. Quantum chaotic scattering in graphene systems in the absence of invariant classical $\begin{array}{ll}\text { dynamics } & 107\end{array}$

F. Conductance stability in chaotic and integrable graphene quantum dots doped with random impurities

G. Chaotic scattering in Dirac fermion systems 113

VI. Role of chaos in relativistic quantum resonant tunneling $\quad 116$

A. Relativistic quantum tunneling - an introduction $\quad 116$

B. Effect of fully developed chaos on relativistic quantum tunneling 117

1. Calculation of tunneling rate in relativistic quantum systems 117

2. Relativistic quantum resonant tunneling in integrable and fully chaotic double $\begin{array}{ll}\text { potential well systems } & 119\end{array}$

C. Relativistic quantum tunneling in nonhyperbolic chaotic systems 120

1. Nonhyperbolic dynamics in the classical limit 120

2. Relativistic quantum manifestation of nonhyperbolic classical dynamics: clustering of tunneling rate 
3. Physical theory for relativistic quantum tunneling in classically nonhyperbolic systems

D. Quantum tunneling in the presence of electron-electron interactions

132

1. Specification of integrable, fully chaotic, and nonhyperbolic domains

133

2. Calculation of resonant tunneling rate in graphene systems described by the Hubbard Hamiltonian

3. Polarization of spin wavefunctions associated with confined states

136

4. Tunneling rate and tunneling probability of spin-polarized states

137

5. Understanding of the emergence of polarized states through mean-field theory

139

6 . Theory of tunneling rate of spin-polarized states

143

7. Regularization of tunneling by chaos

145

8. Remarks on the interplay among chaos, relativistic quantum mechanics, and many-body effects

VII. Superpersistent current in chaotic Dirac fermion systems

150

A. Persistent currents in quantum systems - an introduction $\quad 150$

B. Superpersistent current in chaotic Dirac fermion systems 151

1. A prototypical model of relativistic AB system 151

2. Whispering gallery modes and superpersistent currents in a chaotic domain $\quad 153$

3. Origin of whispering gallery modes and superpersistent currents 156

C. Experimental scheme and robust relativistic qubit 160

D. Robustness of persistent currents in two-dimensional Dirac systems with disorders

VIII. Conclusion and Discussion 166

A. Concluding remarks 166

B. Unsolved problems in relativistic quantum chaos at the present 167

1. Relativistic quantum-classical correspondence 167

2. Semiclassical theory for relativistic quantum scars $\quad 167$

3. Interplay between Klein tunneling and classical dynamics 168

4. Effect of many body interactions on relativistic quantum chaos 169

5. Interplay between spin and nonlinear dynamics 169

6. Relativistic quantum chaos in pseudospin-1 systems $\quad 170$

7. Role of chaos in valley filtering $\quad 171$

8. Interplay between classical chaos and topological quantum phases $\quad 171$

9. Role of chaos in time-dependent, relativistic quantum systems 172

$\begin{array}{ll}\text { IX. Acknowledgement } & 173\end{array}$

$\begin{array}{ll}\text { References } & 174\end{array}$ 


\section{INTRODUCTION}

\section{A. Background: the traditional field of quantum chaos}

The development of nonlinear dynamics and chaos theory in the past half century has revolutionized our understanding of a large variety of phenomena in natural and engineering systems. The hallmark of chaos is sensitive dependence on initial conditions, which lead to random dynamical behaviors even in a completely deterministic system. From the very beginning of the development of nonlinear dynamics, search for manifestations of classical chaos in quantum mechanical systems has attracted the attention of generations of physicists and engineers. Because the fundamental equations in quantum systems, nonrelativistic or relativistic, are linear, generally a sensitive dependence on initial conditions cannot be expected. As such, in typical situations, "real" chaos cannot arise in quantum systems. Sir Michael Berry famously stated [1]: "There is no quantum chaos, in the sense of exponential sensitivity to initial conditions, but there are several novel quantum phenomena which reflect the presence of classical chaos. The study of these phenomena is quantum chaology." However, for historical reasons, the term "quantum chaos" has prevailed. It is now widely agreed that the field of quantum chaos is referred to as the study of the manifestations or fingerprints of classical chaos in quantum systems [2,3], with absolutely no implication that there would be actual chaos in quantum systems. In this Review, we take the liberty to refer to the traditional field of quantum chaos as the study of classical chaos in nonrelativistic quantum systems described by the Schrödinger equation with a parabolic relation between energy and momentum.

Sir Michael Berry made many seminal contributions to the fundamentals of quantum chaos [1, 4-6]. Chirikov conceived and studied the chaotic properties of the standard map [7, 8], a paradigm in the study of Hamiltonian systems and quantum chaos with applications to a large variety of physical systems. Bohigas and collaborators uncovered the phenomenon of level repulsion, attributed its origin to classical chaos, and developed an understanding of the statistics of level spacings based on random matrix ensembles [9]. Casati, Chirikov, Guarneri, and Shepelyansky made significant contributions to our understanding of energy level statistics $[10,11]$ and quantum behaviors of classically driven chaotic systems, a representative class being ionization of excited hydrogen atoms by microwave fields [12-25]. Kaufman and Heller discovered the phenomenon of quantum scarring [26-30]. Prange and Fishman uncovered Anderson localization in kicked quantum chaotic systems [31-33]. Smilansky and Blümel pioneered the area of quantum chaotic scattering [34-41].

Depending on whether the system under study is closed or open, there are different topics in the traditional field of quantum chaos. For example, in closed chaotic Hamiltonian systems, the basic phenomena that have been and continue to be studied include energy level-spacing statistics [4, 5, 9-11, 35, 41-51] and quantum scarring [1, 26-29, 52-79]. In open Hamiltonian systems, quantum chaotic scattering has been investigated extensively [36-40, 80-90]. In addition, the phenomena of quantum diffusion and wavefunction localization in classically driven chaotic systems have been studied [8, 12-23, 31, 91, 92].

This comprehensive Review is about the recently emergent field of Relativistic Quantum Chaos (RQC) - the study of the manifestations or fingerprints of classical chaos in relativistic quantum systems described by the Dirac equation. Interestingly, RQC brings together concepts from the three major scientific revolutions of the twentieth century: relativity, quantum mechanics and 
chaotic dynamics. At the outset, we emphasize that RQC should not be confused with the study of chaos in classical relativistic systems. The main objective of this Review is to address the questions of why, what, and how, through a number of physically significant contexts such as energy level statistics, scarring, scattering, tunneling, and persistent currents, all in the relativistic quantum realm.

\section{B. Relativistic quantum chaos: why, what, and how?}

\section{Why is it important to study RQC?}

Berry and Mondragon were the first to study (about thirty years ago) the energy level-spacing statistics of a classically chaotic system in the relativistic quantum regime - the neutrino billiard system governed by the massless Dirac equation [93]. For such a system, the energy is linearly proportional to the momentum or the wave vector. In a subsequent work by Antoine et al. [94], a 2D fermion billiard in a curved space coupled with a magnetic field was studied. In Ref. [95], Tomaschitz investigated relativistic quantum chaos in open Robertson-Walker cosmologies and found localized wave fields, which are solutions of the Klein-Gordon equation quantized on the bounded trajectories associated with the classical geodesic motion. There were also a few early works on the dynamics of relativistic kicked rotors (the so-called solvable Maryland model) [9698]. In spite of these earlier works, a systematic investigation of RQC was motivated by the tremendous development of two-dimensional (2D) Dirac materials in recent years [99, 100] such as graphene [101-107], topological insulators [108, 109], molybdenum disulfide $\left(\mathrm{MoS}_{2}\right)$ [110, 111], HITP [ $\left.\mathrm{Ni}_{3}(\mathrm{HITP})_{2}\right]$ [112], and topological Dirac semimetals [113, 114]. A common feature of these materials is that their energy bands contain a Dirac cone structure, so they are described by the Dirac equation, generating phenomena that are not usually seen in conventional metallic or semiconductor materials. For example, from the standpoint of quantum transport, the Dirac cone structure and the resulting pseudospin characteristics of the underlying quasiparticles can lead to unconventional physical properties/phenomena such as high carrier mobility, anti-localization, chiral tunneling, and negative refractive index in the optical analog. With the rapid growth of research on 2D Dirac materials, there are needs to uncover, understand, and exploit relativistic quantum manifestations of classical nonlinear dynamical behaviors, including chaos [115-150]. From an applied point of view, due to the underlying physics being effectively governed by the Dirac equation, purely relativistic quantum phenomena such as Klein tunneling, Zitterbewegung, and pair creations can potentially occur in solid state devices and be exploited for significantly improving or even revolutionizing conventional electronics and spintronics. Investigating the manifestations of classical chaos in relativistic quantum systems is thus important with both fundamental and applied values.

\section{What has been done in $R Q C$ ?}

In RQC, topics that have been studied so far include energy level-spacing statistics in graphene systems [115-120], relativistic quantum scarring [121-124], relativistic quantum chaotic scattering and transport [125-133], quantum resonant tunneling in Dirac fermion and graphene systems [134-136], effects of chaos and random disorder on persistent currents in Dirac fermion sys- 
tems [137-139], the role of classical dynamics in confinement of massless Dirac fermions [140143], chaos and spin transport in graphene quantum dot systems [144], and the interplay among chaos, relativistic quantum mechanics, and many body interaction in graphene billiards [136, 145].

\section{How was previous research done in $R Q C$ ?}

A basic requirement is to solve the Dirac equation subject to various physical constraints such as infinite mass confinement and the presence of an external magnetic field. For example, in order to study the energy level statistics for chaotic neutrino billiards, the method of boundary integrals was effective [93]. The eigenenergies and eigenstates of a Dirac fermion in an arbitrarily closed domain can also be solved by using the standard finite element method [122]. For certain chaotic domains, the method of conformal mapping can provide accurate solutions with high computational efficiency $[5,123,151]$. This mapping method can also be extended to situations where an external magnetic flux is present [137]. For graphene systems, open or closed, the tight-binding Hamiltonian and the method of Green's function can be effective, even for the mean-field Hubbard Hamiltonian that includes electron-electron interactions. As research in RQC advances, more diverse, efficient, and powerful methods will surely emerge.

In terms of theoretical analysis, because of the irregular domains required for generating classical chaos, analytic treatment has been limited to approximately describing the corresponding physical situation and to gaining insights from solutions of the Dirac equation in simple settings such as a particle incident on a potential step. Semiclassical theory can also provide useful physical insights into the interplay between chaos and relativistic quantum mechanics.

\section{Outline of this Review}

Our objective is to provide a comprehensive review of the results obtained so far in RQC so as to introduce the emergent field to the scientific community. The discussions will be centered about two types of systems: massless Dirac fermion systems and graphene. While the main task is to present the relativistic quantum manifestations of chaos, in some cases classically integrable dynamics will also be discussed in depth for the purposes of comparison and contrast. We will first introduce the existing solution methods for research in RQC. We will then discuss physical phenomena pertinent to the interplay between chaos and relativistic quantum mechanics, which include energy level statistics, scarring, chaotic scattering and transport, resonant tunneling, and persistent currents.

Solution methods in RQC. For graphene systems, closed or open, the tight-binding method is standard. For closed systems (i.e., graphene billiards), the eigenenergies and eigenstates can be found through diagonalizing the tight-binding Hamiltonian. For open systems (e.g., graphene quantum dots), the method of non-equilibrium Green's function constructed from the tight-binding Hamiltonian can be used to calculate various quantities characterizing quantum transport. We will describe the boundary conditions for graphene billiards and discuss how electron-electron interactions can be treated in the tight-binding framework. We will also describe how microwave billiards with a triangular photonic structure can be exploited as an experimental scheme to investigate various phenomena in graphene billiards.

For Dirac fermion systems, methods to solve the 2D Dirac equation in arbitrarily geometric 
domains that exhibit a spectrum of classical dynamics ranging from integrable to chaotic are necessary. We will first describe the Dirac equation and the unique boundary conditions. Next we will present a finite element method for solving the Dirac equation in two dimensions, focusing on specific issues such as the discretization scheme, elimination of the fermion doubling effect, boundary conditions, matrix representation of the Dirac equation, and solution validation using toy models for which the Dirac equation is analytically solvable. We will then discuss the boundary integral and conformal mapping methods for solving the 2D massless Dirac equation, without or with an external magnetic field. Especially, we will detail the solution method for a ring domain subject to a central magnetic flux, elaborating on issues such as the effects of the magnetic flux on the energy spectra, analytic solutions for an integrable domain, and conformal mapping solutions for chaotic domains. Finally, we will present a number of illustrative examples.

Energy level-spacing statistics in relativistic quantum chaotic systems. In traditional quantum chaos, a fundamental and well studied topic is the statistics of energy level spacings in systems with classical chaotic dynamics $[2,3,152]$. A well established result is that, for classically chaotic systems without any geometrical symmetry, the level-spacing statistics can be described by those of random matrices [4, 9, 153]. For example, for systems with a time-reversal symmetry, the statistics are characteristic of those of random matrices from the Gaussian orthogonal ensemble (GOE) [2]. If, in addition to time-reversal symmetry, the system has a half-integer spin interaction, the resulting level-spacing statistics follow those of random matrices from the Gaussian symplectic ensemble (GSE). When the time-reversal symmetry is broken, e.g., as in the presence of a magnetic field, the level-spacing statistics are governed by the Gaussian unitary ensemble (GUE) random matrices.

We will review the relevant works on level-spacing statistics for both graphene billiards with confined pseudo-Dirac quasiparticles and two-dimensional massless Dirac billiards, with a special eye toward the fundamental issue of whether the distinct statistical behaviors associated with different symmetry classes are preserved in relativistic quantum chaotic systems, since some basic traits such as intrinsic spin, linear dispersion, Klein tunneling, and chirality, etc., are not present in nonrelativistic quantum systems [154]. The work of Berry and Mondragon [93] on the massless neutrino billiard was seminal, where they found that, if the classical dynamics are integrable, the level-spacing statistics are of the Poisson type - a result similar to that in nonrelativistic quantum systems. However, for classically chaotic dynamics, the level-spacing statistics are those of GUE, even in the absence of any magnetic field. Berry and Mondragon attributed this peculiar phenomenon to the chiral nature of Dirac particles and the scalar confinement potential that breaks the time-reversal symmetry. Graphene confinements that have the geometric shape of chaotic billiards represent a class of experimental systems for testing energy-level statistics in the relativistic quantum regime.

Relativistic quantum scars. Scarring is a remarkable phenomenon in quantum systems whose classical dynamics are chaotic. Intuitively, because of ergodicity associated with chaos, one may expect the quantum wavefunctions to be uniformly distributed in both the position and phase spaces. Signatures of highly non-uniform distribution of the wave functions, in particular their unusually high concentrations about the classically unstable periodic orbits, were first discovered by McDonald and Kaufman in their computation of the eigenstates of a quantum particle confined in the chaotic stadium billiard [26], a phenomenon that was later coined as "quantum scars" by Heller [28]. The phenomenon of scarring was then explained theoretically by Bogomolny [53] and Berry [1] based on the semiclassical Green's function. In traditional quantum chaos, scars 
have been an active area of research [27, 29, 52, 54-79]. In open systems such as quantum dots, the remnants of scars exist: quantum pointer states [73, 155-160] that can lead to significant conductance fluctuations associated with electronic transport [69, 80, 81, 161-163].

We will present a systematic review of the phenomenon of scarring in relativistic quantum systems, focusing on such issues as whether scars can arise in relativistic quantum systems and whether there are unique characteristics of relativistic quantum scars that have no counterparts in nonrelativistic quantum systems. In particular, we will discuss results from a study of chaotic graphene billiards [121], where relativistic quantum scars were found. We will then present a striking class of scars, chiral scars, which occur on classical periodic orbits with an odd number of bounces off the domain boundary. For such a scar, the phase difference of a full rotation around the periodic orbit and its time reversed counterpart will gain an additional value of $\pi$, leading to anomalous half values of a semiclassical characterizing quantity. Chiral scars were discovered, thanks to the development of a conformal-mapping based method to solve the Dirac equation with infinite mass confinement, which enables accurate and efficient determination of a large number of energy levels and the associated eigenstates [123]. We will also provide a detailed account of the fundamental physical mechanisms for chiral scars based on a combination of considerations of symmetry and the effect of an external magnetic field.

Relativistic quantum chaotic scattering. Chaotic scattering is a well studied phenomenon in classical nonlinear dynamics [164-170], which is the physical manifestation of transient chaos in classical Hamiltonian systems [171]. In open Hamiltonian systems, scattering dynamics can range from being regular to nonhyperbolic with a mixed phase space, and to fully developed (hyperbolic) chaotic.

In traditional quantum chaos, the scattering matrix (S-matrix) is the standard tool to characterize quantum chaotic scattering, whose elements are the transition amplitudes between quantum states of the system before and after the scattering, which is largely attributed to Miller's work that expressed the S-matrix in terms of purely classical quantities [172]. For fully developed classical chaos, the S-matrix elements in the semiclassical regime exhibit random fluctuations [173] with respect to parameter variations, where it was found that hyperbolic and nonhyperbolic scattering can have quite distinct quantum manifestations in the statistical properties of the fluctuations of the S-matrix elements [82].

We will review several key results in relativistic quantum chaotic scattering, focusing on the problem of conductance fluctuations in graphene quantum dot systems. We will demonstrate that classical chaos can suppress sharp resonances and smooth out the fluctuations with energy. The idea of exploiting chaos to control the statistical properties of conductance fluctuations will be discussed.

Role of chaos in relativistic quantum tunneling. The interplay between classical chaos and quantum tunneling has been a topic of continuous interests [174-181]. Among the many interesting phenomena, chaos-regularized quantum resonant tunneling [180] is remarkable, where classical chaos can significantly suppress the spread in the tunneling rate commonly seen in classically regular systems. Resonant tunneling typically occurs in a closed billiard system (often symmetric) with a localized potential barrier separating the cavity into two parts. For an infinite barrier, each cavity is a closed system with an infinite set of eigenenergies and eigenstates. If the classical dynamics are integrable, some stable periodic orbits whose trajectories do not encounter the potential barrier lead to quantum states with extremely low tunneling rate even when the energy is comparable with or larger than the height of the potential barrier. There are also eigenstates 
corresponding to classical orbits that interact with the potential barrier, and these are strongly tunneling states. As a result, the quantum tunneling rate can spread over a wide range. For classically chaotic dynamics, typical orbits interact with the potential barrier and this effectively removes the extremely small values of the tunneling rate and suppresses its spread.

We will first discuss relativistic quantum resonant tunneling in systems with fully developed chaos. We will then describe the effect of classically nonhyperbolic dynamics on tunneling. Finally, we will address the issue of many body effects by presenting some key results on resonant tunneling in chaotic graphene systems modeled by the mean-field Hubbard Hamiltonian that includes electron-electron interactions. A unique phenomenon that finds no counterpart in nonrelativistic quantum tunneling systems is that, due to Klein tunneling, even when the particle energy is much smaller than the potential height, there can still be a significant amount of quantum tunneling. The main message is that, classical chaos can effectively regularize quantum resonant tunneling, regardless of the nature of the tunneling system (e.g., graphene or Dirac fermion) and regardless of whether many body interactions are taken into account.

Superpersistent current in chaotic Dirac fermion systems. Currents requiring no external voltage with zero resistance, persistent or permanent currents, can arise in systems that are not superconducting. This remarkable phenomenon was theoretically predicted [182] to occur in normal metallic or semiconductor ring systems subject to a central Aharonov-Bohm (AB) [183] magnetic flux. Persistent currents were indeed observed experimentally in metallic [184-188] and semiconductor [189-191] rings in the mesoscopic regime. In nonrelativistic quantum systems, material impurities, random disorder, or boundary imperfection can have a devastating effect on persistent currents, fundamentally restricting the phenomenon to systems at or below the mesoscopic scale.

Boundary irregularities can lead to classical chaos even in a circular domain - a prototype of quantum systems in which persistent currents are typically studied. In nonrelativistic quantum systems, boundary deformation leading to chaos can diminish the currents [192-198]. However, it was discovered recently that persistent currents can arise and sustain in the presence of symmetrybreaking perturbations in classically chaotic Dirac fermion systems [137]. The underlying physical mechanism was identified to be Dirac whispering gallery modes (WGMs) along the domain boundary, which carry large angular momenta. It was also found that in Dirac fermion systems, persistent currents are robust against random disorders [139]. The focus of our discussion will then be on the robustness of persistent currents in relativistic quantum systems with classical chaos or random disorders. 


\section{RELATIVISTIC QUANTUM BILLIARDS AND SOLUTION METHODS}

\section{A. Graphene billiard}

\section{Tight-binding Hamiltonian}

Graphene billiards represent a paradigmatic setting to study the relativistic quantum manifestations of classical chaos [115, 121, 147]. A graphene billiard, or a graphene flake, is a confinement with abrupt edge terminations. Ideally, it is equivalent to a two-dimensional infinite potential well of certain geometric shape: the potential is zero within the confinement region but it is infinite outside of the region. The shape of the region can be chosen to yield distinct classical dynamics including chaos.

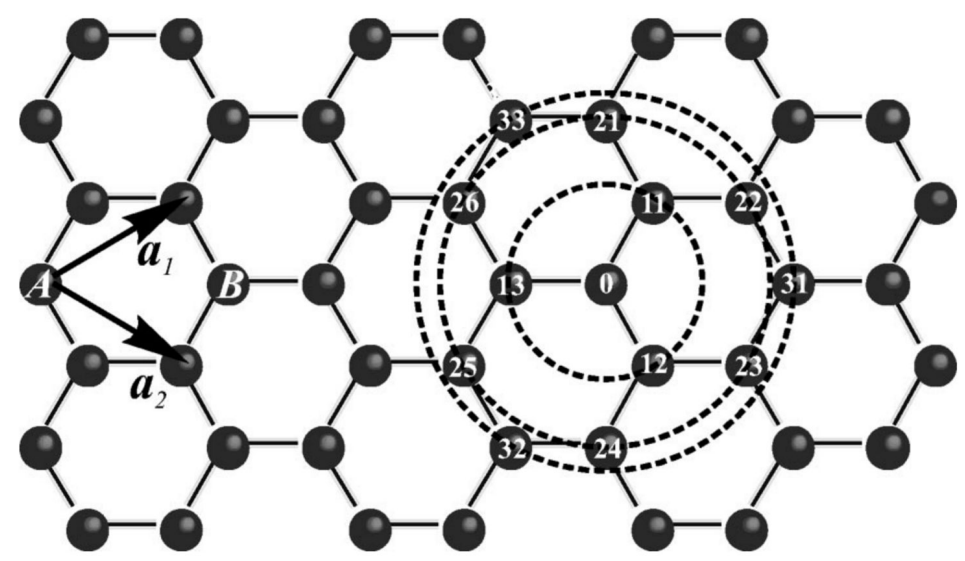

FIG. 1. Graphene hexagonal lattice. The unit cell contains two carbon atoms $A$ and $B$ belonging to two nonequivalent sublattices, where $\boldsymbol{a}_{1}$ and $\boldsymbol{a}_{2}$ are the unit cell vectors with lattice constant $a=2.461 \AA$. Atom 0 has three nearest neighbors: $11-13$, six second-nearest neighbors: $21-26$, and three third-nearest neighbors: $31-33$. The horizontal and vertical edges correspond to armchair and zigzag boundaries, respectively. From Ref. [199] with permission.

An accurate description of the electronic band structure of graphene requires the value of the hopping energies between an atom and its nearest, next-nearest, and second-nearest neighbors, as shown in Fig. 1. The band structure so calculated agrees well with that from ab initio calculations [199]. A peculiar feature is the existence of the Dirac point where the two bands touch linearly and form a Dirac cone, where the quasiparticles follow the 2D massless Dirac equation. Nonetheless, if one is interested in the behavior of the quasiparticles only close to the Dirac point, considering only the nearest neighbor interactions suffices. For a graphene confinement in the presence of a magnetic field, the tight-binding Hamiltonian taking into account only the nearest neighbor hopping energy is given by

$$
\hat{H}=\sum\left(-t_{i j}\right)|i\rangle\langle j|,
$$

where the summation is over all pairs of nearest neighboring atoms, and

$$
t_{i j}=t \exp \left(-i \frac{2 \pi}{\Phi_{0}} \int_{\boldsymbol{r}_{j}}^{\boldsymbol{r}_{i}} \mathrm{~d} \mathbf{r} \cdot \boldsymbol{A}\right),
$$


is the nearest-neighbor hopping energy, $\boldsymbol{A}$ is the vector potential associated with the magnetic field, $\Phi_{0}=h / e=4.136 \times 10^{-15} \mathrm{Tm}^{2}$ is the magnetic flux quantum, $h$ is the Planck constant and $e$ is the electron charge, and $t \approx 2.8 \mathrm{eV}$ is the nearest-neighbor hopping energy in the absence of a magnetic field [101-107]. Under the Landau gauge, the vector potential can be written as $\boldsymbol{A}=(-B y, 0,0)$ for a uniform magnetic field $B$ perpendicular to the billiard plane. It is often convenient to use the magnetic flux $\Phi=B S$ through a hexagonal plaque as a parameter characterizing the magnetic field, where the area is $S=3 \sqrt{3} / 2 a_{0}^{2}=5.24 \AA^{2}$, and $a_{0}=1.42 \AA$ is the atomic separation distance of the graphene lattice. The on-site energy can be neglected because it has approximately the same value for all carbon atoms in the graphene confinement. The eigenenergies and the eigenstates can be calculated through diagonalization of the tight-binding Hamiltonian.

\section{Boundary conditions for graphene billiards}

At low energies, the quasiparticle motions in graphene obey relativistic Dirac equation. To solve the Dirac equation in an arbitrary two-dimensional domain, the boundary conditions are of critical importance. It is thus insightful to examine the boundary conditions for a graphene domain, where the graphene lattice can be terminated in an arbitrary orientation (see Eqs. (3.8) and (3.9) in Ref. [200]). In the summation form in Eq. (3.10) there, only the terms with $\left|\lambda_{ \pm}\right|=1$ form the four-component spinor in the Dirac equation, and the remaining terms describe how the boundary modes decay from an edge. Through such an analysis, the authors of Ref. [200] found that, for most orientations, the boundary conditions should be of the zigzag type. The staggered boundary potential in graphene mimics the mass confinement for Dirac particles, leading to a boundary conditions that reside on an extreme point opposite to the zigzag one. This leads to certain difference between the boundary conditions for a confined Dirac domain and for a terminated graphene lattice.

A graphene system in the absence of magnetic field preserves the time-reversal symmetry, which is the starting point to derive the boundary conditions [200]. In particular, the staggered lattice potential breaks the pseudo-spin symmetry of the sublattice, which resembles the symplectic symmetry of a spin system, but preserves the true time-reversal symmetry. A unique characteristic of graphene systems is pseudospins due to the $A$ and $B$ atoms in a unit cell that constitute two inter-displaced triangular lattices, respectively. This is a feature that is not reflected by the conventional Dirac equation that describes the behavior of a single relativistic quantum particle. As a result, for relativistic quantum systems described by the Dirac equation, the mass term (not necessarily infinite) breaks the true time reversal symmetry. This difference between a graphene system and a Dirac fermion system can manifest itself in the statistics of energy level-spacing in classically chaotic billiards, where a chaotic graphene billiard exhibits GOE (Gaussian Orthogonal Ensemble) statistics $[115,118,120]$, while a chaotic Dirac billiard such as the neutrino billiard has the GUE (Gaussian Unitary Ensemble) statistics [93], as to be discussed extensively in Sec. III.

The boundary conditions for quasiparticle motions in a graphene system were obtained using the Dirac equation [200], taking into account the discrete-lattice structure. A complete description of graphene incorporating boundaries requires four-component spinors due to the necessity to include the pseudospin quantum numbers associated with the two sublattices and the two nondegenerate valleys. Solutions of the 2D Dirac equation with two-component spinors are thus appropriate only to describe the relativistic quantum motion of pseudo-particles in graphene only in the absence of inter-valley scatterings. In general, the boundaries of a closed graphene domain 
will mix the quantum dynamics associated with the two valleys, implying that the two-component Dirac equation cannot provide a completely accurate description of graphene systems. Nevertheless, analysis and solutions of the 2D Dirac equation in an arbitrary geometrical shape can provide insights into the behaviors of the graphene billiard of the same shape and understanding of phenomena such as chaotic scattering [132, 140, 141, 201].

\section{Interacting electrons in graphene billiard}

In condensed matter systems, many body interactions (or electron-electron interactions) lead to fascinating phenomena such as superconductivity and fractional quantum Hall effect. In the field of quantum chaos, the standard setting was that of single-particle quantum dynamics, in which many-body effects were ignored. While there were previous studies of the interplay between many-body interactions and classical chaos [202-205], these were exclusively for non-relativistic quantum systems. To investigate the effect of chaos on relativistic quantum systems with manybody interactions is thus an outstanding problem not only fundamental to physics but also important for the practical development of relativistic quantum devices.

The interplay between classical dynamics and many-body interactions in the specific setting of relativistic quantum tunneling in graphene systems can be studied [136] using the celebrated Hubbard model [206]. The main contribution to $\pi$-electron hopping in the graphene honeycomb lattice is $p_{z}$ orbital, where each orbital can have two electrons at most, one spin up and another spin down. To gain insights into the essential physics of electron-electron interactions in graphene while still making the model tractable, one needs to take into account nearest-neighbor hopping terms and electron Coulomb repulsion at the local site. The tight-binding Hubbard Hamiltonian has the following standard form [207]:

$$
\hat{H}=-t \sum_{\langle i, j\rangle, \sigma} c_{i, \sigma}^{\dagger} c_{j, \sigma}+U(x, y) \sum_{i, \sigma} c_{i, \sigma}^{\dagger} c_{i, \sigma}+U_{H} \sum_{i, \sigma} n_{i, \sigma} n_{i, \bar{\sigma}},
$$

where the summation of $\langle i, j\rangle$ is over all nearest-neighbor pairs, the index $\{\sigma, \bar{\sigma}\}$ denotes spin up and down electrons, $c_{i, \sigma}^{\dagger}\left(c_{j, \sigma}\right)$ is the creation (annihilation) operator, $n_{i \sigma}=c_{i, \sigma}^{\dagger} c_{i, \sigma}$ is the number operator, $U(x, y)$ is the location dependent external electric potential, and $U_{H}$ is the Coulomb energy describing the interaction between a spin-up and a spin-down electrons at the same site. While the Hubbard Hamiltonian provides a somewhat simplified picture of electron-electron interactions in the corresponding system, the analysis and computations become extremely difficult even for moderate system size with only tens of atoms. For relatively large system size, approximation must be employed to gain physical understanding of the system behaviors. A standard approach is to use the mean-field approximation, where the Hamiltonian (3) is modified to [208-213]

$$
\hat{H}_{M F}=-t \sum_{\langle i, j\rangle, \sigma} c_{i, \sigma}^{\dagger} c_{j, \sigma}+U(x, y) \sum_{i, \sigma} c_{i, \sigma}^{\dagger} c_{i, \sigma}+U_{H} \sum_{i, \sigma}\left\langle n_{i, \bar{\sigma}}\right\rangle n_{i, \sigma} .
$$

Physically, the mean-field Hamiltonian describes the situation where a spin-up electron at site $i$ interacts with the average spin-down electron population $\left\langle n_{i \downarrow}\right\rangle$ at the same site, and vice versa. The mean field Hubbard model is effectively a variation of the unrestricted Hartree-Fock approximation [214]. There have been recent efforts in comparing the various aspects of the mean-field Hubbard model with those from first-principle or quantum Monte-Carlo calculations, with the 
conclusion that the mean-field approximation is generally valid for graphene systems [212, 213], especially in the weakly coupling regime $[209,215]$. It is thus reasonable to choose the parameter $U_{H}$ below the critical Coulomb repulsion [213] $U_{c}=2.2 t$.

System (4) can be solved iteratively, as follows. At half-filling and zero temperature, the average density of electrons with spin $\sigma$ at atom $i$ is defined as $\left\langle n_{i, \sigma}\right\rangle=\sum_{n=1}^{N / 2} \rho_{i, \sigma}\left(E_{n}\right)$, where $N$ is the total number of eigenstates with a given spin, and $\rho_{i, \sigma}\left(E_{n}\right)=\left|\psi_{i, \sigma}^{n}\right|^{2}$ is the local density of states (LDS) at site $i$ for the $n$ 'th eigenstate $\psi_{i, \sigma}^{n}$. Starting from an initial condition of $\left\langle n_{i, \bar{\sigma}}\right\rangle$ for spin $\bar{\sigma}$, one has the complete Hamiltonian in (4) for spin $\sigma$, which yields a new set of eigenstates $\left\{E_{n}, \psi_{\sigma}^{n}, n=1, \cdots, N\right\}$ and can then be used to calculate $\rho_{\sigma}\left(E_{n}\right)$, leading to a new set of $\left\langle n_{i, \sigma}\right\rangle$. Using $\left\langle n_{i, \sigma}\right\rangle$ as the initial condition, one can use the Hamiltonian in (4) to solve the set of eigenstates for spin $\bar{\sigma}$, yielding a new set of average density $\left\langle n_{i, \bar{\sigma}}\right\rangle$ of electrons for spin $\bar{\sigma}$. One then iterates the process until $\left\langle n_{i, \sigma}\right\rangle$ and $\left\langle n_{i, \bar{\sigma}}\right\rangle$ reach a steady state.

A concrete example is resonant tunneling in a closed graphene billiard, where the whole domain is divided into two sub-domains: the left and right, with a 1D potential barrier at the boundary [136]. The initial configuration can be conveniently chosen to be that of an anti-ferromagnetic state, where the initial values of $\left\langle n_{i \downarrow}\right\rangle$ are chosen to be $+1 /-1$ at sublattice $A / B$, respectively. The local spin density at site $i$ is $m_{i}=\left(\left\langle n_{i, \uparrow}\right\rangle-\left\langle n_{i, \downarrow}\right\rangle\right) / 2$. In the Hubbard model, the number of electrons is fixed, so the total spin density of the whole system is given by $M=\sum_{i=1}^{N} m_{i}=$ $M^{L}+M^{R}=0$, where $M^{L}$ and $M^{R}$ represent the total spin density at the left and the right sides of the potential barrier, respectively.

\section{B. Microwave billiards with a triangular photonic structure}

Artificial honeycomb lattices offer a tunable platform for studying massless Dirac quasiparticles. There have been extensive works on constructing such lattices employing nanopatterning of two-dimensional electron gases in semiconductors, molecule-by-molecule assembly by scanning probe methods, and optical trapping of ultracold atoms in crystals of light (Ref. [216], references therein). Due to the wave nature of the electron motion in the honeycomb graphene lattice, the relativistic pseudo-particles can also be created using microwave photonic crystals [217-230] or mechanical vibrations and phononic crystals [231-236], where the propagation of the electromagnetic or acoustic wave follows the same honeycomb lattice structure, as shown in the left panel of Fig. 2. This leads to remarkable behaviors in tunneling and edge states [228-230].

TABLE I. Coupling parameters in GHz and overlapping parameters for the tight-binding model as fitted from the density of eigenfrequencies. From Ref. [224] with permission.

\begin{tabular}{cccccccc}
\hline \hline & $\gamma_{0}$ & $\gamma_{1}$ & $\gamma_{2}$ & $\gamma_{3}$ & $s_{1}$ & $s_{2}$ & $s_{3}$ \\
\hline B1 & 0.011 & 1.009 & 0.022 & -0.013 & -0.060 & -0.023 & -0.009 \\
B2 & 0.007 & 1.008 & 0.038 & -0.011 & -0.062 & -0.020 & 0.002 \\
\hline \hline
\end{tabular}

Based on the honeycomb lattice structure of the voids between the metal cylinders (left panel of Fig. 2), one can employ the tight-binding method to calculate the band structure of the microwave photonic crystal [199, 237], as shown in the right panel of Fig. 2. In particular, the dependence of the frequencies $f(\boldsymbol{q})$ on the wave vector $\boldsymbol{q}$ can be obtained by solving the following generalized 

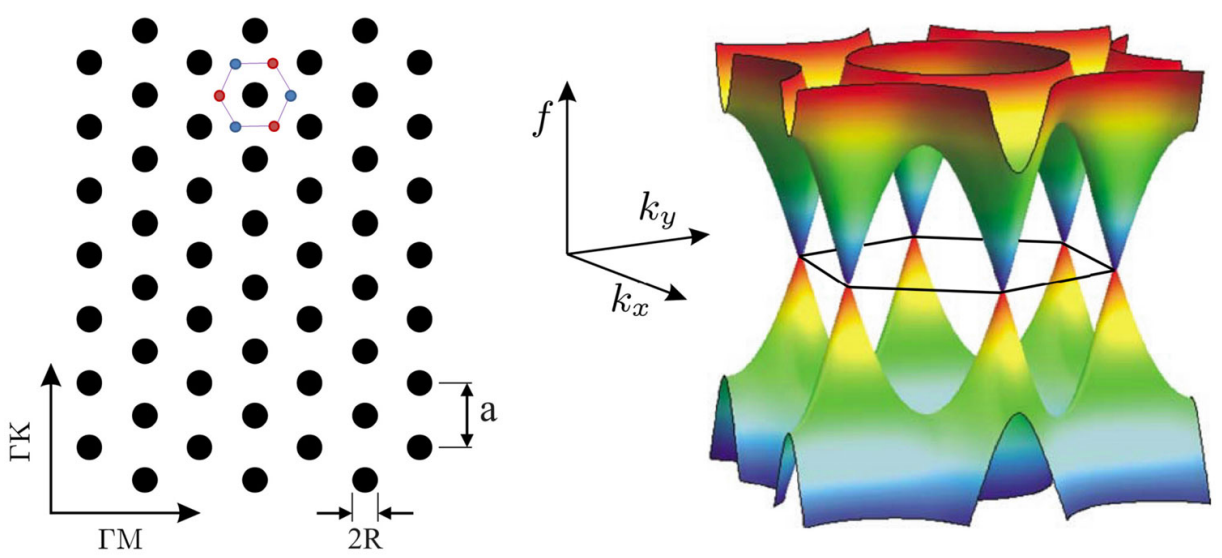

FIG. 2. Triangular lattice of metallic cylinders and the corresponding band structure. Left: a triangular lattice of metallic cylinders, where $a$ is the lattice constant and $R=0.25 a$. The voids (small blue and red circles) between the cylinders form the honeycomb lattice. Right: band structure for microwave propagating in the honeycomb lattice of voids. Modified from Ref. [217] with permission.

eigenvalue problem [224]:

$$
\hat{H}_{T B}\left|\psi_{\boldsymbol{q}}(\boldsymbol{r})\right\rangle=f(\boldsymbol{q}) S_{W O}\left|\psi_{\boldsymbol{q}}(\boldsymbol{r})\right\rangle
$$

with the tight-binding Hamiltonian

$$
\hat{H}_{T B}=\left(\begin{array}{cc}
\gamma_{0}+\gamma_{2} h_{2}(\boldsymbol{q}) & \gamma_{1} h_{1}(\boldsymbol{q})+\gamma_{3} h_{3}(\boldsymbol{q}) \\
\gamma_{1} h_{1}(\boldsymbol{q})+\gamma_{3} h_{3}(\boldsymbol{q}) & \gamma_{0}+\gamma_{2} h_{2}(\boldsymbol{q})
\end{array}\right)
$$

and the wavefunction overlapping matrix is given by

$$
S_{W O}=\left(\begin{array}{cc}
1+s_{2} h_{2}(\boldsymbol{q}) & s_{1} h_{1}(\boldsymbol{q})+s_{3} h_{3}(\boldsymbol{q}) \\
s_{1} h_{1}(\boldsymbol{q})+s_{3} h_{3}(\boldsymbol{q}) & 1+s_{2} h_{2}(\boldsymbol{q})
\end{array}\right)
$$

where $\gamma_{0}$ is the on-site potential, $\gamma_{n}(n=1,2,3)$ are the nearest-, second-nearest- and thirdnearest-neighbor coupling energies, $s_{n}(n=1,2,3)$ are the corresponding overlaps between the wavefunctions centered at different sites, $h_{n}(\boldsymbol{q})(n=1,2,3)$ are associated with the different coupling energies [199]. In Ref. [224], the authors investigated superconducting microwave Dirac billiards (e.g., left panels of Fig. 3) and obtained the density of eigenfrequencies experimentally (black curves in the right panels of Fig. 3). The tight-binding model was used to fit the data, yielding the values of the coupling and overlapping parameters as listed in Table I. From the right panels of Fig. 3, one can see that the tight-binding model and the experimental results agree very well, indicating the validity and accuracy of the tight-binding model. A different work using artificial honeycomb lattice of dielectric resonators gave similar results for the coupling parameters [221].

Chaotic Dirac billiard of microwave photonic crystal can be constructed by setting the boundaries such that the classical dynamics are chaotic. For example, an Africa shaped superconducting microwave photonic crystal was constructed and the spectral statistics were investigated [225, 227]. 

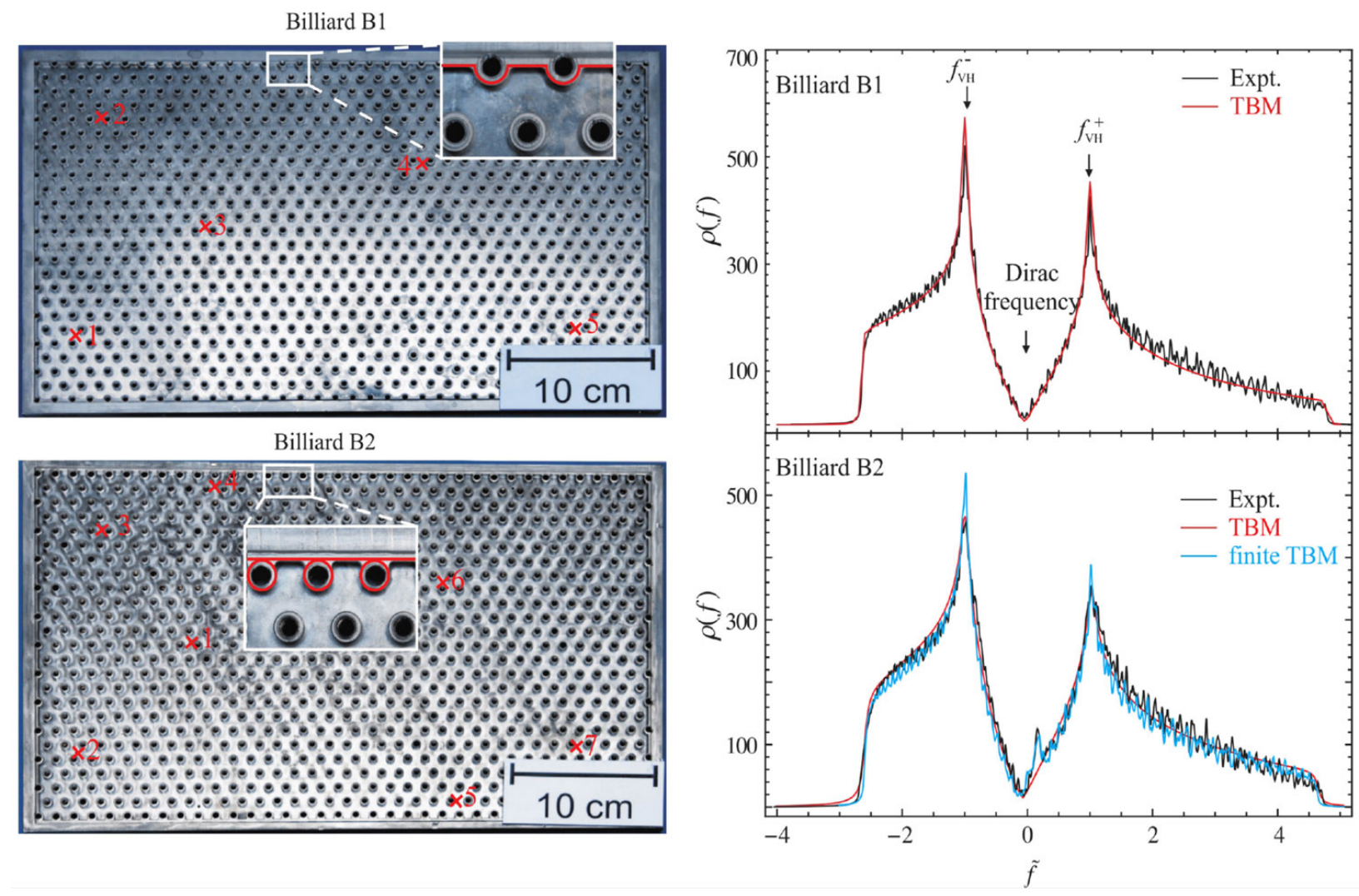

FIG. 3. Basin plates of microwave Dirac billiards and the corresponding density of states. Left: two billiards with different boundaries, each containing 888 metal cylinders. Right: the corresponding density of eigenfrequencies from experiments and from an infinite size tight-binding model. Lower panel: results from the tight-binding model of the same size as the experimental system. Modified from Ref. [224] with permission.

\section{Dirac billiard}

\section{Dirac equation in a closed domain}

Consider a massless spin-half particle in a finite domain $D$ in the plane $\boldsymbol{r}=(x, y)$, as shown in Fig. 4. Utilizing an infinite-mass term outside the domain to model the confinement of the particle motion within $D$, one can write the Hamiltonian in the position representation as

$$
\hat{H}=-i \hbar c \hat{\boldsymbol{\sigma}} \cdot \nabla+V(\boldsymbol{r}) \hat{\sigma}_{z}
$$

where $c$ is the speed of light (when describing pseudo-Dirac particles in 2D Dirac materials, $c$ should be replaced by the corresponding Fermi velocity $\left.v_{F}\right), \hat{\boldsymbol{\sigma}}=\left(\hat{\sigma}_{x}, \hat{\sigma}_{y}\right)$ and $\hat{\sigma}_{z}$ are Pauli matrices, and $V(\boldsymbol{r})$ is the infinite-mass confinement potential:

$$
V(\boldsymbol{r})=\left\{\begin{array}{cc}
0, & \boldsymbol{r} \in D, \\
\infty, & \boldsymbol{r} \text { outside of } D .
\end{array}\right.
$$




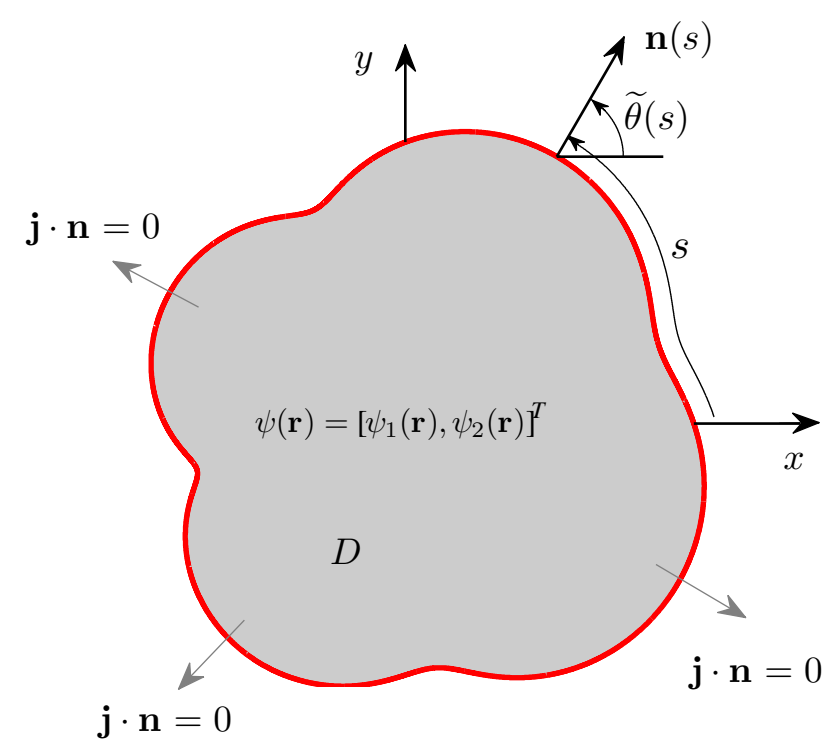

FIG. 4. Illustration of a 2D Dirac billiard domain and the boundary conditions. The domain boundary is parametrized by arc length $s$. For motion of a massless Dirac fermion inside the domain, the boundary conditions are of the zero-flux type, i.e., no outward current at any point $s: \boldsymbol{j} \cdot \boldsymbol{n}=0$, which is equivalent to $\psi_{2} / \psi_{1}=i \exp [i \widetilde{\theta}(s)]$ with $\widetilde{\theta}(s)$ being the argument of the outward normal $\boldsymbol{n}(s)$.

The Hamiltonian $\hat{H}$ acts on the two-component spinor wave-function $\psi(\boldsymbol{r})=\left[\psi_{1}, \psi_{2}\right]^{\mathrm{T}}$ and it has eigenenergy $E$, i.e.,

$$
\left[-i \hbar c \hat{\boldsymbol{\sigma}} \cdot \boldsymbol{\nabla}+V(\boldsymbol{r}) \hat{\sigma}_{z}\right] \psi(\boldsymbol{r})=E \psi(\boldsymbol{r}) .
$$

Some basic properties of Eq. (8) are the following. Firstly, the confinement condition of imposing infinite mass outside $D$ naturally overcomes the difficulty for electric potential caused by Klein tunneling for relativistic quantum particles. Secondly, the reduced spatial dimension and confinement break the time-reversal symmetry of $\hat{H}$, namely

$$
[\hat{\mathcal{T}}, \hat{H}] \neq 0
$$

where $\hat{\mathcal{T}}=i \sigma_{y} \hat{K}$, and $\hat{K}$ denotes complex conjugate. Thirdly, for $V=0$ in Eq. (8), there exist plane-wave solutions whose positive-energy part has the form

$$
\psi_{\boldsymbol{k}}(\boldsymbol{r})=\frac{1}{\sqrt{2}}\left(\begin{array}{c}
\exp \left(-i \frac{\theta}{2}\right) \\
\exp \left(i \frac{\theta}{2}\right)
\end{array}\right) \exp (i \boldsymbol{k} \cdot \boldsymbol{r})
$$

where $\boldsymbol{k}$ is a wave-vector that makes an angle $\theta$ with the $x$ axis.

In the presence of a magnetic field, the Dirac Hamiltonian is

$$
\hat{H}=c \hat{\boldsymbol{\sigma}} \cdot(-i \hbar \boldsymbol{\nabla}+e \boldsymbol{A})+V(\boldsymbol{r}) \hat{\sigma}_{z}
$$

where $\boldsymbol{A}$ is the vector potential. 


\section{Boundary conditions for Dirac billiards}

To obtain solutions of Eq. (8), one needs a proper treatment of the boundary conditions, which is a subtle and challenging issue [200]. Due to the finite domain and the first-order nature of the Dirac equation, a naive treatment of the boundary conditions will lead to trivial or even nonphysical solutions. One example is the relativistic particle in a one-dimensional box. By simply letting the whole spinor have zero values at the walls of the box, only a trivial (all-zero) solution of the eigenfunctions can be obtained. To overcome this difficulty, many self-adjoint extensions of the boundary conditions in both Dirac and Weyl representations have been proposed [238]. For example, in $(1+1)$ dimensions, one family of boundary conditions is to force either the large or the small component of the spinor to be zero at the walls of the box. Some variances of these boundary conditions also exist [239, 240], e.g., by assuming that the large component vanishes at one boundary and the small component vanishes at the other, or by assuming that both components differ by factors $\pm i$. Some of these boundary conditions also preserve the physical symmetries, such as $\mathcal{P}$ and $\mathcal{C P} \mathcal{T}$ symmetries. However, these types of conditions are not appropriate in $(2+$ 1 ) dimensions, because the walls of the box are impenetrable. Physically, this means that the relativistic current $\boldsymbol{j}=c \psi^{\dagger} \hat{\boldsymbol{\sigma}} \psi$ normal to the boundaries must vanish. The vanishing current condition was previously used in the bag model [241-243] of quark confinement, which solves the Dirac equation with a Lorentz scalar potential. It was assumed that the rest mass of the particle $m(\boldsymbol{r})$ is a position-dependent parameter. One could then solve this infinite-well problem for a particle of varying mass by letting the mass go to infinity outside the box in order to take into account the Klein paradox. A similar method was adopted by Berry and Mondragon [93] in their study of the energy level-spacing statistics for the relativistic neutrino billiard.

A proper treatment of the boundary conditions requires the inclusion of a mass term in the Dirac Hamiltonian. Even for a massless particle inside the billiard, the boundary confinement can be thought of that of an infinite mass potential. It is thus useful to examine the general Dirac equation for a massive particle in $(2+1)$ dimensions, which is given by

$$
i \hbar \partial_{t} \psi(t)=\hat{H} \psi(t)
$$

where the general form of the Hamiltonian is

$$
\hat{H}=c(\hat{\boldsymbol{\alpha}} \cdot \hat{\boldsymbol{p}})+\hat{\beta} m c^{2},
$$

and $\psi$ is a two-component Dirac spinor. Assuming stationary solution $\psi(t)=\psi \exp (-i E t / \hbar)$, one obtains the steady-state Dirac equation

$$
\hat{H} \psi=E \psi
$$

In two dimensions, $\hat{\boldsymbol{\alpha}}=\hat{\boldsymbol{\sigma}}=\left(\sigma_{x}, \sigma_{y}\right)$ and $\beta=\sigma_{z}$ are choices satisfying all anticommutation/commutation relations of the Dirac/Lorentz algebra [244].

Two methods are available for obtaining the proper boundary conditions: either replacing the $m c^{2} \sigma_{z}$ term by a potential $V(\boldsymbol{r}) \sigma_{z}$ in the Hamiltonian and letting $V(\boldsymbol{r})$ tend to infinity outside the domain, or using the vanishing current condition $\boldsymbol{j} \cdot \boldsymbol{n}=0$, where $\boldsymbol{n}$ is the normal vector at the boundary, as shown in Fig. 4. The second method leads to

$$
\operatorname{Re}\left(e^{i \widetilde{\theta}(s)} \psi_{1} / \psi_{2}\right)=0
$$


where $\phi$ and $\chi$ are the components of the Dirac spinor, $\psi=\left[\psi_{1}, \psi_{2}\right]^{T}$, and $\widetilde{\theta}(s)$ is the argument of the surface normal $\boldsymbol{n}$. The boundary condition can then be written as [93]

$$
\psi_{2} / \psi_{1}=i \exp [i \tilde{\theta}(s)] .
$$

When an external electric potential $U$ is present, $E$ is replaced by $E-U$. For a massless fermion, one can then write the Dirac equation as

$$
[c(\hat{\boldsymbol{\sigma}} \cdot \hat{\boldsymbol{p}})+U] \psi=E \psi,
$$

where $c$ is the speed of light which, for graphene, should be replaced by the Fermi velocity $v_{F} \sim$ $10^{6} \mathrm{~m} / \mathrm{s}$.

\section{Finite element method for solving the Dirac equation in two dimensions}

\section{Discretization scheme and elimination of fermion doubling effect}

To numerically solve the Dirac equation, it is necessary to develop an efficient and physically meaningful discretization scheme. Unlike the standard discretization of second order differential equations such as the Schrödinger equation, discretization for the massless Dirac equation is a much harder problem. A difficulty is that the usual finite difference methods fail because they introduce the so-called fermion-doubling effect, even for open or periodic boundaries. Fermion doubling is also a problem for lattice QCD computations [241-243].

The phenomenon of fermion doubling can be explained conveniently using the Dirac equation in one dimension:

$$
i \hbar \partial_{t} \psi=i \hbar c \sigma_{x} \partial_{x} \psi
$$

Setting the lattice grid $x=n \Delta$ and exploiting the central difference approximation

$$
\partial_{x} \psi(n)=[\psi(n+1)-\psi(n-1)] /(2 \Delta),
$$

one obtains the following Fourier transformed equation:

$$
i \hbar \partial_{t} \tilde{\psi}=\left[\hbar c \sigma_{x} \sin \left(p_{x} \Delta\right) / \Delta\right] \tilde{\psi}=\tilde{H} \tilde{\psi} .
$$

The energy is given by

$$
|E|=\left|\hbar c \sigma_{x} \sin \left(p_{x} \Delta\right) / \Delta\right| .
$$

In the first Brillouin zone (BZ) where $p \in[-\pi / \Delta, \pi / \Delta]$, the energy expression indicates that there are more than one point satisfying the linear energy-momentum relation, giving rise to fermion doubling. There were previous efforts in eliminating this effect [245, 246].

To eliminate fermion doubling, one can design certain discretization scheme $[122,246]$ for solving the 2D Dirac equation, as shown in Fig. 5. One first discretizes the whole domain using a two-dimensional lattice. The Dirac spinors are evaluated at lattice points $(m, n)$. One then solves the Dirac equation at the center of each unit cell: $\left(m+\frac{1}{2}, n+\frac{1}{2}\right)$. From the Hamiltonian, one has that the derivatives of the Dirac spinor are approximately given by

$$
\begin{aligned}
& \partial_{x} \psi_{m+\frac{1}{2}, n+\frac{1}{2}}=\frac{\psi_{m+1, n+1}+\psi_{m+1, n}-\psi_{m, n+1}-\psi_{m, n}}{2 \Delta}, \\
& \partial_{y} \psi_{m+\frac{1}{2}, n+\frac{1}{2}}=\frac{\psi_{m+1, n+1}+\psi_{m, n+1}-\psi_{m+1, n}-\psi_{m, n}}{2 \Delta} .
\end{aligned}
$$




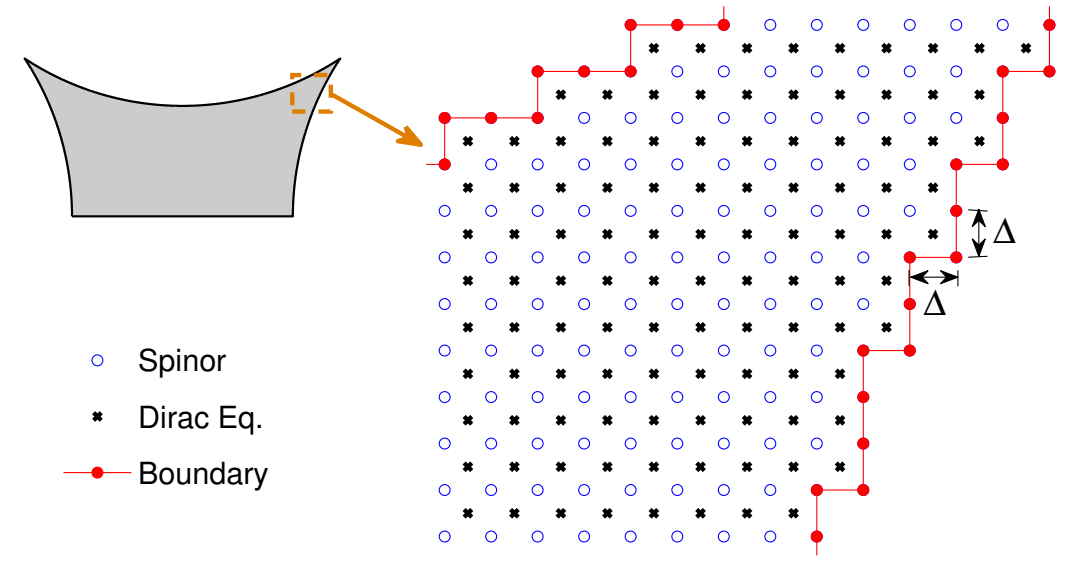

FIG. 5. A discretization scheme for solving the Dirac equation in an arbitrary closed 2D domain with fermion doubling eliminated. A two-dimensional bowtie domain, which exhibits chaos in the classical limit, is illustrated to show the discretized lattice. The red filled and blue open circles spaced by $\Delta$ represent the boundary and inner lattice points, respectively, where the Dirac spinor values are sampled. The actual Dirac equations are evaluated at the black cross points, the centers of unit cells. From Ref. [122] with permission.

The spinors at the unit cell centers can be approximated as the average of the four spinor values from the neighboring lattice points:

$$
\psi_{m+\frac{1}{2}, n+\frac{1}{2}}=\frac{1}{4}\left(\psi_{m+1, n+1}+\psi_{m, n+1}+\psi_{m+1, n}+\psi_{m, n}\right) .
$$

It was demonstrated [122] that this numerical scheme can successfully eliminate fermion doubling.

\section{Boundary conditions and matrix representation of Dirac equation}

In a closed domain, the number of Dirac equations at unit cell centers (denoted as $M$ ) is less than the number of total spinors at the lattice points (denoted as $N$ ): $M<N$. The difference can be accounted for by the boundary conditions. It is necessary to write the Dirac equation in matrix form to incorporate the boundary conditions into the solution procedure. Let $\boldsymbol{\psi}=\left(\psi_{1}, \psi_{2}, \ldots, \psi_{N}\right)^{T}$ be the column vector containing all spinor values on the lattice, where $\boldsymbol{\psi}$ actually has $2 N$ components. Let $\boldsymbol{D}_{x} /(2 \Delta), \boldsymbol{D}_{y} /(2 \Delta)$ and $\boldsymbol{A} / 4$ be the matrix representation of the operators $\partial_{x}, \partial_{y}$, and the averaging operator in Eq. (20), respectively. These matrices all have the dimension $M \times N$. In the matrix form, Eq. (16) becomes [122]

$$
\left[-\frac{2 i \hbar c}{\Delta}\left(\boldsymbol{D}_{x} \otimes \sigma_{x}+\boldsymbol{D}_{y} \otimes \sigma_{y}\right)+U(x, y) \boldsymbol{A} \otimes \mathbf{1}_{2}\right] \psi=E \boldsymbol{A} \otimes \mathbf{1}_{2} \psi \text {. }
$$

Since there are $2 M$ equations, $2 N-2 M$ boundary conditions are needed, which can be written as

$$
\boldsymbol{B} \boldsymbol{\psi}=0
$$


where $\boldsymbol{B}$ is a $(2 N-2 M) \times 2 N$ matrix. Since not all spinors are independent, the spinor vector can be permuted by

$$
\boldsymbol{\psi}^{\prime}=\boldsymbol{P} \boldsymbol{\psi}=\left[\begin{array}{l}
\boldsymbol{\psi}_{D} \\
\boldsymbol{\psi}_{B}
\end{array}\right]
$$

where $\psi_{D}$ 's are independent Dirac spinors and $\psi_{B}$ 's are spinors at the boundary that can be expressed by other components in $\boldsymbol{\psi}_{D}$, and $\boldsymbol{P}$ is an orthogonal permutation matrix. Defining

$$
\boldsymbol{H}^{\prime}=\left[-\frac{2 i \hbar c}{\Delta}\left(\boldsymbol{D}_{x} \otimes \sigma_{x}+\boldsymbol{D}_{y} \otimes \sigma_{y}\right)+U(x, y) \boldsymbol{A} \otimes \mathbf{1}_{2}\right] \boldsymbol{P}^{T}
$$

$\boldsymbol{A}^{\prime}=\boldsymbol{A} \otimes{ }_{2} \boldsymbol{P}^{T}$, and $\boldsymbol{B}^{\prime}=\boldsymbol{B} \boldsymbol{P}^{T}$, one obtains

$$
\boldsymbol{H}^{\prime} \psi^{\prime}=E \boldsymbol{A}^{\prime} \psi^{\prime}, \quad \boldsymbol{B}^{\prime} \psi^{\prime}=0 .
$$

With the boundary conditions, one can explicitly express $\boldsymbol{\psi}_{B}$ by $\boldsymbol{\psi}_{D}$. Let $\boldsymbol{B}^{\prime}=\left[\boldsymbol{B}_{1}, \boldsymbol{B}_{2}\right]$, where $\boldsymbol{B}_{2}$ is a square matrix. One has

$$
\boldsymbol{\psi}_{B}=-\boldsymbol{B}_{2}^{-1} \boldsymbol{B}_{1} \boldsymbol{\psi}_{D}
$$

Letting $\boldsymbol{H}^{\prime}=\left[\boldsymbol{H}_{1}, \boldsymbol{H}_{2}\right]$ and $\boldsymbol{A}^{\prime}=\left[\boldsymbol{A}_{1}, \boldsymbol{A}_{2}\right]$, where $\boldsymbol{H}_{2}$ and $\boldsymbol{A}_{2}$ are square matrices, and substituting $\boldsymbol{\psi}_{B}$ into $\boldsymbol{H}^{\prime} \psi^{\prime}=E \boldsymbol{A}^{\prime} \psi^{\prime}$, one finally obtains

$$
\boldsymbol{H}_{D} \boldsymbol{\psi}_{D}=E \boldsymbol{\psi}_{D}
$$

where

$$
\boldsymbol{H}_{D}=\left(\boldsymbol{A}_{1}-\boldsymbol{A}_{2} \boldsymbol{B}_{2}^{-1} \boldsymbol{B}_{1}\right)^{-1}\left(\boldsymbol{H}_{1}-\boldsymbol{H}_{2} \boldsymbol{B}_{2}^{-1} \boldsymbol{B}_{1}\right) .
$$

An issue with the newly defined Hamiltonian $\boldsymbol{H}_{D}$ is that it is not Hermitian in general due to the finite domain and the lattice approximation of the original smooth boundaries, in spite of its possessing real eigenvalues. This difficulty can be overcome through introducing

$$
\boldsymbol{H}=\left(\boldsymbol{H}_{D}+\boldsymbol{H}_{D}^{\dagger}\right) / 2
$$

which is the Hamiltonian for a new physical system. For infinitesimal values of $\Delta$, the energy spectra of the two systems are identical, and the eigenstates of the two systems are close to each other especially at low energies, where the discretized system is a faithful representation of the Dirac equation.

3. Validation of solution method using a ring domain for which the Dirac equation is analytically solvable

Even for a domain as simple as a rectangle, the Dirac equation is not analytically solvable due to the entanglement of the two Cartesian coordinates. The ring domain provides a setting in which the variables can be separated so that the eigenvalues and eigenstates can be obtained analytically, enabling test and validation of the numerical solution method. The general solutions of the Dirac equation in a domain with circular boundaries can be written as

$$
\psi_{n}=N e^{i n \theta}\left[\begin{array}{c}
Z_{n}(k r) \\
\operatorname{sgn}(E-U) i e^{i \theta} Z_{n+1}(k r)
\end{array}\right], n=0, \pm 1, \ldots
$$




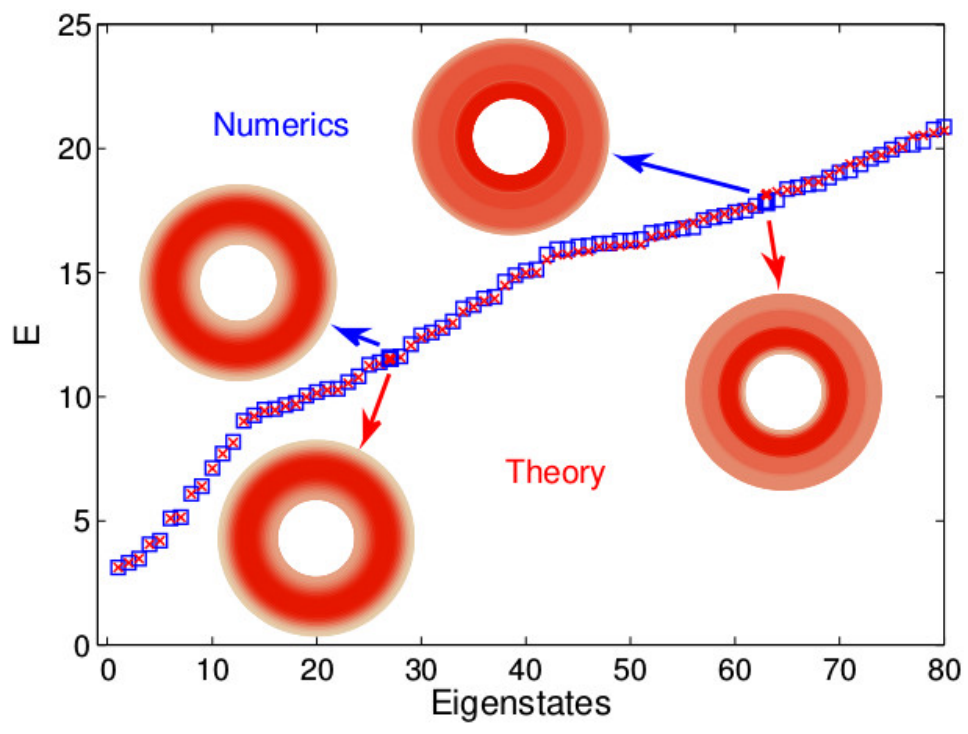

FIG. 6. Comparison of numerical and exact eigenenergies and eigenstates of Dirac equation in a ring billiard. For a ring domain, the Dirac equation is analytically solvable, which provides a system to test and validate the numerical solution procedure. The lowest 80 positive eigenenergy levels and two examples of the eigenstates from numerics (blue squares) and theory (red crosses) are compared. The unit convention $\hbar=c=1$ was used in the numerical computation. From Ref. [122] with permission.

where $k=|E-U| /(\hbar c)$, and $N$ is a normalization constant. For a ring domain, $Z_{n}(x)$, the radial function of the spinor components, is a linear combination of the first- and second-kind Bessel functions, $J_{n}(x)$ and $Y_{n}(x)$. Say the inner and outer radii of the ring domain are $R_{1}=0.5$ and $R_{2}=1$, respectively. Analytically, the solution in Eq. (27) can be obtained with a potential that has a staircase-like profile in the radial direction but is constant in the angular direction. However, the case with an arbitrary potential can only be solved numerically. For the simple case where the electrical potential is zero, the energy levels for each angular mode can be obtained using the inner and outer boundary conditions, $E_{m}^{(n)}=\hbar c k_{m}^{(n)}$, where $k_{m}^{(n)}$ can be obtained as the solutions of

$$
\begin{aligned}
& {\left[J_{n+1}\left(k R_{1}\right)+J_{n}\left(k R_{1}\right)\right]\left[Y_{n+1}\left(k R_{2}\right)-Y_{n}\left(k R_{2}\right)\right] } \\
= & {\left[J_{n+1}\left(k R_{2}\right)-J_{n}\left(k R_{2}\right)\right]\left[Y_{n+1}\left(k R_{1}\right)+Y_{n}\left(k R_{1}\right)\right] . }
\end{aligned}
$$

The eigenstates can be calculated after the normalization constants $N_{m}^{(n)}$ are computed. Results of the analytical calculations in comparison with the numerical solutions are demonstrated in Fig. 6 - there is essentially no discrepancy.

\section{E. Boundary integral method for solving the Dirac equation in two dimensions}

The relativistic version of the boundary integral technique was first developed by Berry and Mondragon for calculating the eigenvalues of the chaotic neutrino billiard [93], which is described by the massless Dirac equation. The general idea is to reduce the problem of $2 \mathrm{D}$ linear differential equation to a $1 \mathrm{D}$ integral equation on the boundary by the Green's function method. 
For a general 2D domain $D$ with boundary $\partial D$, the massless Dirac equation under the unit convention $\hbar=c=1$ is

$$
-i \hat{\boldsymbol{\sigma}} \cdot \nabla \psi(\boldsymbol{r})=k \psi(\boldsymbol{r}),
$$

where $\psi(\boldsymbol{r})=\left[\psi_{1}, \psi_{2}\right]^{T}$ and the boundary condition is of the vanishing outward current type that is equivalent to [93]

$$
\frac{\psi_{2}(\boldsymbol{r})}{\psi_{1}(\boldsymbol{r})}=i \exp \{i \widetilde{\theta}(s)\}
$$

at the boundary point $\boldsymbol{r} \in \partial D$, with $\widetilde{\theta}(s)$ denoting the angle between the horizon and the outward unit normal vector $\boldsymbol{n}$, as shown in Fig. 7. The Dirac equation in Eq. (29) can be reduced to an integral equation about the boundary $\partial D$ by means of the free-space Green's function $G^{0}\left(\boldsymbol{r}, \boldsymbol{r}^{\prime} ; k\right)$ defined as

$$
k G^{0}\left(\boldsymbol{r}, \boldsymbol{r}^{\prime} ; k\right)+i \hat{\boldsymbol{\sigma}} \cdot \nabla_{\boldsymbol{r}} G^{0}\left(\boldsymbol{r}, \boldsymbol{r}^{\prime} ; k\right)=\delta\left(\boldsymbol{r}-\boldsymbol{r}^{\prime}\right) \mathbf{1}
$$

In general, a reflection correction $G^{1}$ is necessary due to the propagator (Green's function) $G^{0}$ from the boundary to the free space. However, it was demonstrated [93] that the term $G^{1}$ has no effect on the eigenvalues. Using $G^{0}$ thus suffices to calculate the eigenvalues through the boundary integral. Letting $\rho=\left|\boldsymbol{r}-\boldsymbol{r}^{\prime}\right|$ and $\left(\partial_{x} \pm i \partial_{y}\right)=e^{ \pm i \tilde{\theta}}\left(\partial_{n} \pm i \partial_{s}\right)$, one has

$$
G^{0}\left(\boldsymbol{r}, \boldsymbol{r}^{\prime} ; k\right)=-\frac{1}{4}\left(\begin{array}{cc}
i k H_{0}^{(1)}(k \rho) & -e^{-i \widetilde{\theta}} H_{1}^{(1)}(k \rho)\left(\partial_{n}-i \partial_{s}\right) \rho \\
-e^{i \widetilde{\theta}} H_{1}^{(1)}(k \rho)\left(\partial_{n}+i \partial_{s}\right) \rho & i k H_{0}^{(1)}(k \rho)
\end{array}\right),
$$

where $H_{\nu}^{(1)}$ with $\nu=0,1$ denotes the $\nu$ 'th-order Hankel function of the first kind.

Letting $\psi^{\dagger}(\boldsymbol{r})=\left(\psi_{1}^{*}, \psi_{2}^{*}\right)$, one has

$$
k \psi^{\dagger}(\boldsymbol{r})-i \boldsymbol{\nabla}_{\boldsymbol{r}} \psi^{\dagger}(\boldsymbol{r}) \cdot \hat{\boldsymbol{\sigma}}=\mathbf{0} .
$$

The operation of $\psi^{\dagger}(\boldsymbol{r}) \times(31)-(33) \times G^{0}\left(\boldsymbol{r}, \boldsymbol{r}^{\prime} ; E\right)$ leads to

$$
i \boldsymbol{\nabla}_{\boldsymbol{r}} \cdot\left(\psi^{\dagger} \hat{\boldsymbol{\sigma}} G^{0}\right)=\delta\left(\boldsymbol{r}-\boldsymbol{r}^{\prime}\right) \mathbf{1}
$$

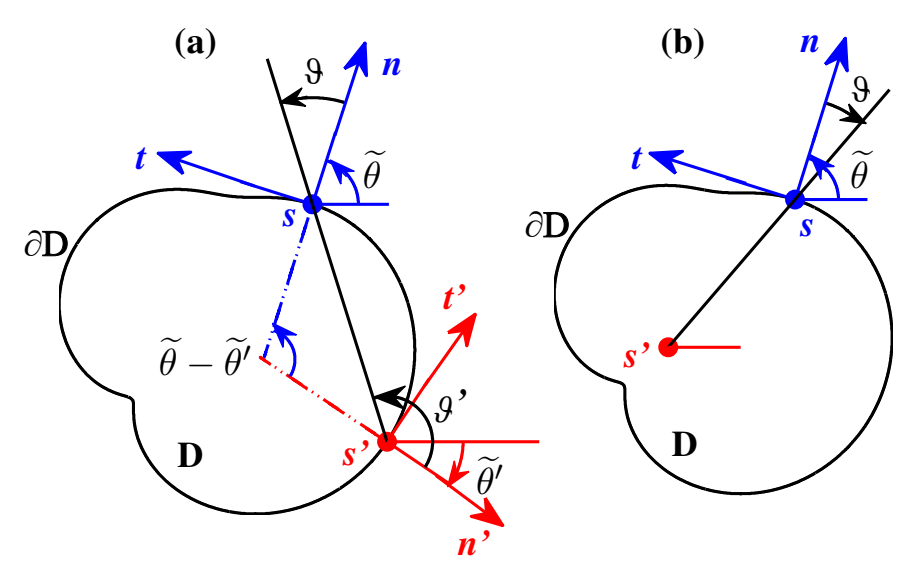

FIG. 7. Basic setting of the boundary integral method for solving the Dirac equation in an arbitrary 2D closed domain. Shown are the various angles and chords used in boundary integral. 
Next, integrating $\boldsymbol{r}$ over $D$ and using Green's theorem yields

$$
i \oint \mathrm{d} s \boldsymbol{n}(s) \cdot\left[\psi^{\dagger} \hat{\boldsymbol{\sigma}} G^{0}\left(\boldsymbol{r}, \boldsymbol{r}^{\prime} ; E\right)\right]= \begin{cases}\psi^{\dagger}\left(\boldsymbol{r}^{\prime}\right) & \left(\boldsymbol{r}^{\prime} \in D\right), \\ \frac{1}{2} \psi^{\dagger}\left(\boldsymbol{r}^{\prime}\right) & \left(\boldsymbol{r}^{\prime} \in \partial D\right), \\ 0 & \left(\boldsymbol{r}^{\prime} \notin D \cup \partial D\right),\end{cases}
$$

where $\boldsymbol{n}(s)=(\cos \widetilde{\theta}(s), \sin \widetilde{\theta}(s))$. Taking $\boldsymbol{r}^{\prime} \in \partial D$ and substituting (32) into (35) yield

$$
\begin{aligned}
-\frac{k}{4} i \oint \mathrm{d} s\left[i \psi_{2}^{*}(s) e^{i \widetilde{\theta}} H_{0}^{(1)}(k \rho)-\psi_{1}^{*}(s) H_{1}^{(1)}(k \rho)\left(\partial_{n}+i \partial_{s}\right) \rho\right] & =\frac{1}{2} \psi_{1}^{*}\left(s^{\prime}\right), \\
-\frac{k}{4} i \oint \mathrm{d} s\left[-\psi_{2}^{*}(s) H_{1}^{(1)}(k \rho)\left(\partial_{n}-i \partial_{s}\right) \rho+i \psi_{1}^{*}(s) e^{-i \widetilde{\theta}} H_{0}^{(1)}(k \rho)\right] & =\frac{1}{2} \psi_{2}^{*}\left(s^{\prime}\right) .
\end{aligned} .
$$

Using the boundary condition (30), one arrives at

$$
\begin{aligned}
\frac{i k}{4} \oint \mathrm{d} s \psi_{1}^{*}(s)\left[H_{1}^{(1)}(k \rho)\left(\partial_{n}+i \partial_{s}\right) \rho-H_{0}^{(1)}(k \rho)\right] & =\frac{1}{2} \psi_{1}^{*}\left(s^{\prime}\right), \\
\frac{i k}{4} \oint \mathrm{d} s \psi_{1}^{*}(s)\left[e^{i\left(\widetilde{\theta^{\prime}}-\widetilde{\theta}\right)} H_{1}^{(1)}(k \rho)\left(\partial_{n}-i \partial_{s}\right) \rho+e^{i\left(\widetilde{\theta}^{\prime}-\widetilde{\theta}\right)} H_{0}^{(1)}(k \rho)\right] & =\frac{1}{2} \psi_{1}^{*}\left(s^{\prime}\right) .
\end{aligned} .
$$

Note that the solution is singular at $s=s^{\prime}$ because of the singularities in the Hankel functions. However, the singularities can be removed [93] straightforwardly by adding the two integral expressions in (37) for $\psi_{1}^{*}\left(s^{\prime}\right)$. One thus obtains the following regularized integral equation,

$$
\begin{aligned}
\psi_{1}^{*}\left(s^{\prime}\right) & =\frac{i k}{4} \oint \mathrm{d} s \psi_{1}^{*}(s)\left\{\left(e^{i\left(\widetilde{\theta^{\prime}}-\widetilde{\theta}\right)}-1\right) H_{0}^{(1)}(k \rho)\right. \\
& \left.+\left[\left(\partial_{n}+i \partial_{s}\right) \rho+e^{i\left(\widetilde{\theta^{\prime}}-\widetilde{\theta}\right)}\left(\partial_{n}-i \partial_{s}\right) \rho\right] H_{1}^{(1)}(k \rho)\right\} .
\end{aligned}
$$

Using the geometric relations as shown in Fig. 7,

$$
\left\{\begin{array}{c}
e^{i \vartheta}=\left(\partial_{n}+i \partial_{s}\right) \rho, \\
\vartheta^{\prime}=\vartheta+\widetilde{\theta}-\widetilde{\theta}^{\prime}
\end{array}\right.
$$

one finally gets

$$
\psi_{1}^{*}\left(s^{\prime}\right)=\oint \mathrm{d} s \psi_{1}^{*}(s)\left\{i M\left(s, s^{\prime}\right) H_{0}^{(1)}(k \rho)+N\left(s, s^{\prime}\right) H_{1}^{(1)}(k \rho)\right\},
$$

where

$$
\left\{\begin{array}{l}
M\left(s, s^{\prime}\right)=M\left(s^{\prime}, s\right)^{*}=\frac{k}{4}\left[e^{i\left(\widetilde{\theta^{\prime}}-\widetilde{\theta}\right)}-1\right], \\
N\left(s, s^{\prime}\right)=N\left(s^{\prime}, s\right)^{*}=\frac{i k}{4}\left[e^{i \vartheta}+e^{-i \vartheta^{\prime}}\right]
\end{array}\right.
$$

and $M$ and $N$ are Hermitian under interchange of $s$ and $s^{\prime}$.

To numerically solve the boundary integral equation (BIE) in Eq. (40), one simply divides the boundary $\partial D$ of length $\mathcal{L}$ equally into $K$ points and defines

$$
\left\{\begin{array}{l}
s_{m} \equiv \frac{m \mathcal{L}}{K} ; \quad \rho\left(s_{l}, s_{m}\right) \equiv \rho_{l m} \\
M\left(s_{l}, s_{m}\right) \equiv M_{l m} ; \quad N\left(s_{l}, s_{m}\right) \equiv N_{l m} \\
\psi_{1}^{*}\left(s_{l}\right) \equiv u_{l} ; \quad l, m=1,2, \cdots, K
\end{array}\right.
$$


so as to convert Eq. (40) into a finite set of linear homogeneous equations

$$
\sum_{l=1}^{K} u_{l}\left\{\delta_{l m}-\frac{\mathcal{L}}{K}\left[i M_{l m} H_{0}^{(1)}\left(k \rho_{l m}\right)+N_{l m} H_{1}^{(1)}\left(k \rho_{l m}\right)\right]\right\}=0 .
$$

Numerically, the eigenvalues $k_{n}$ are approximately the zeros of the $K \times K$ complex determinant

$$
\mathcal{D}_{K}(k)=\operatorname{det}\left\{\delta_{l m}-\frac{\mathcal{L}}{K}\left[i M_{l m} H_{0}^{(1)}\left(k \rho_{l m}\right)+N_{l m} H_{1}^{(1)}\left(k \rho_{l m}\right)\right]\right\} .
$$

If the boundary is not divided equally, a factor accounting for the length of each segment is necessary in Eqs. (42-44).

To give an example, consider the family of analytical boundary defined by

$$
x(\phi)+i y(\phi)=w(z) \equiv w\left(e^{i \phi}\right),
$$

with the following analytic relations associated with $w(z)$ on the boundary:

$$
\left\{\begin{aligned}
g(z) & =\frac{\mathrm{d} w}{\mathrm{~d} z} ; \quad \mathrm{d} s=\sqrt{\mathrm{d} x^{2}+\mathrm{d} y^{2}}=|g(z)| \mathrm{d} \phi \\
e^{i \widetilde{\theta}} & =-i \frac{\mathrm{d} x+i \mathrm{~d} y}{\mathrm{~d} s}=\frac{z g(z)}{|g(z)|} ; \quad \rho=\left|w(z)-w\left(z^{\prime}\right)\right|, \\
e^{i \vartheta} & =e^{-i \widetilde{\theta}}\left(\partial_{x}+i \partial_{y}\right) \rho=\frac{z^{*} g(z)^{*}\left(w(z)-w\left(z^{\prime}\right)\right)}{|g(z)| \rho} \\
e^{-i \vartheta^{\prime}} & =e^{-i \vartheta} e^{i\left(\widetilde{\theta}^{\prime}-\widetilde{\theta}\right)}=\frac{z^{\prime} g\left(z^{\prime}\right)\left(w(z)-w\left(z^{\prime}\right)\right)^{*}}{\left|g\left(z^{\prime}\right)\right| \rho}
\end{aligned}\right.
$$

Equations (42-44) can be rewritten in a more numerically feasible form as

$$
\left\{\begin{aligned}
\phi_{l} & =\frac{2 \pi l}{K} ; \quad \phi_{m}=\frac{2 \pi m}{K} ; \quad 1 \leq l, m \leq K, \\
z_{l} & \equiv e^{i \phi_{l}} ; \quad w_{l} \equiv w\left(z_{l}\right) ; \quad g_{l} \equiv g\left(z_{l}\right), \\
\rho_{l m} & =\left|w_{l}-w_{m}\right|, \\
M_{l m} & =\frac{z_{l}^{*} z_{m} g_{l}^{*} g_{m}}{\left|g_{l} g_{m}\right|}-1, \\
N_{l m} & =i\left\{\frac{z_{l}^{*} g_{l}^{*}\left(w_{l}-w_{m}\right)}{\left|g_{l}\right| \rho_{l m}}+\frac{z_{m} g_{m}\left(w_{l}-w_{m}\right)^{*}}{\left|g_{m}\right| \rho_{l m}}\right\}, \\
\mathcal{D}_{K}(k) & =\operatorname{det}\left\{\delta_{l m}-\frac{\pi k}{2 K} \sqrt{\left|g_{l} g_{m}\right|}\left[i M_{l m} H_{0}^{(1)}\left(k \rho_{l m}\right)+N_{l m} H_{1}^{(1)}\left(k \rho_{l m}\right)\right]\right\} .
\end{aligned}\right.
$$

A few remarks on the boundary integral method are in order. Firstly, while it can be shown [247] that the discrete linear equation converges exactly to the continuous boundary integral equation in the limit $K \rightarrow \infty, K$ must be finite in actual computations. It is thus necessary to estimate how large $K$ must be. To obtain an efficient numerical approximation to an eigenvalue $k$ corresponding to the de Broglie wavelength $\lambda=2 \pi / k$, one can introduce the density $d$ of discretization defined as the number of discrete points per de Broglie wavelength on the boundary: $d=K \lambda / \mathcal{L}$. Thus 
the numerical criterion for calculating $k$ is $d \gg 1$ (e.g., $d \sim 10$ in practice). Secondly, as demonstrated, a merit of the boundary integral method is to convert the problem in two dimensions to one in one dimension so as to dramatically reduce the amount of the computation for a desired accuracy [248]. The boundary integral method has been widely used not only in studying quantum chaos, but also in engineering [249]. For most eigenenergies, the results agree well with those from other methods, e.g., the conformal mapping method to be discussed next.

\section{F. Conformal mapping method for solving the Dirac equation in two-dimensional chaotic bil- liards}

The idea of employing conformal mapping to calculate quantum eigenenergies and eigenstates was first proposed by Robnik and Berry $[5,151]$ for nonrelativistic quantum billiard systems. This idea was recently extended to solving the Dirac equation in relativistic quantum billiard systems without a magnetic field [123] or with an Aharonov-Bohm magnetic flux [124, 137]. In particular, a class of chaotic billiards can be obtained through conformal mapping from a circular domain, which allows a large number of eigenvalues and eigenstates of the former to be calculated with high precision, leading to the discovery of chiral scars in relativistic quantum chaotic systems [123, 124] (see Chap. IV). When a perpendicular magnetic flux is present at the center of a chaotic ring domain, the conformal mapping method enables accurate calculation of the persistent currents associated with different eigenstates [137] (see Chap. VII).

The basic idea underlying the conformal mapping method is that, while the Dirac equation together with the boundary condition is generally not separable in the Cartesian coordinates, for a circularly symmetric disk/ring domain, analytic solutions can be written down, which form a complete orthonormal basis. Given a closed domain with an analytic boundary, if a proper conformal mapping exists that can transform the domain into a circular disk/ring, the analytic solutions of the latter can be exploited to yield the solutions of the former. Before the transform, the geometrical space within the domain is flat, but there are irregularities on the boundary which lead to random or chaotic scattering. After the transformation, the boundary becomes simple, e.g., a circle, but the equation itself becomes complex with the underlying geometrical space becoming inhomogeneous. Since a complete orthonormal basis is available for the circular domain, the wavefunction can be expanded onto this basis. Solving the expansion coefficients yields the eigenenergy and eigenwavefuctions. The solutions in the circular disk/ring can then be transformed back to the original chaotic domain to yield a large number of eigenvalues and eigenstates with high accuracy.

\section{Analytic solution of the Dirac equation in the circular domain}

In the polar coordinates $\boldsymbol{r}=(r, \phi)$, for a closed circular domain of radius $r_{0}$, the Dirac equation Eq. (6) takes the form

$$
-i\left(\begin{array}{cc}
0 & \exp (-i \phi)\left(\frac{\partial}{\partial r}-\frac{i}{r} \frac{\partial}{\partial \phi}\right) \\
\exp (i \phi)\left(\frac{\partial}{\partial r}+\frac{i}{r} \frac{\partial}{\partial \phi}\right) & 0
\end{array}\right) \psi(\boldsymbol{r})=\mu \psi(\boldsymbol{r}),
$$

where $\mu \equiv E / \hbar c$. For a circular boundary, the following relation holds: $\left[\hat{J}_{z}, \hat{H}\right]=0$, where $\hat{J}_{z}=-i \partial_{\phi}+1 / 2 \hat{\sigma}_{z}$ is the total angular momentum operator. In general, the solutions of Eq. (48) 


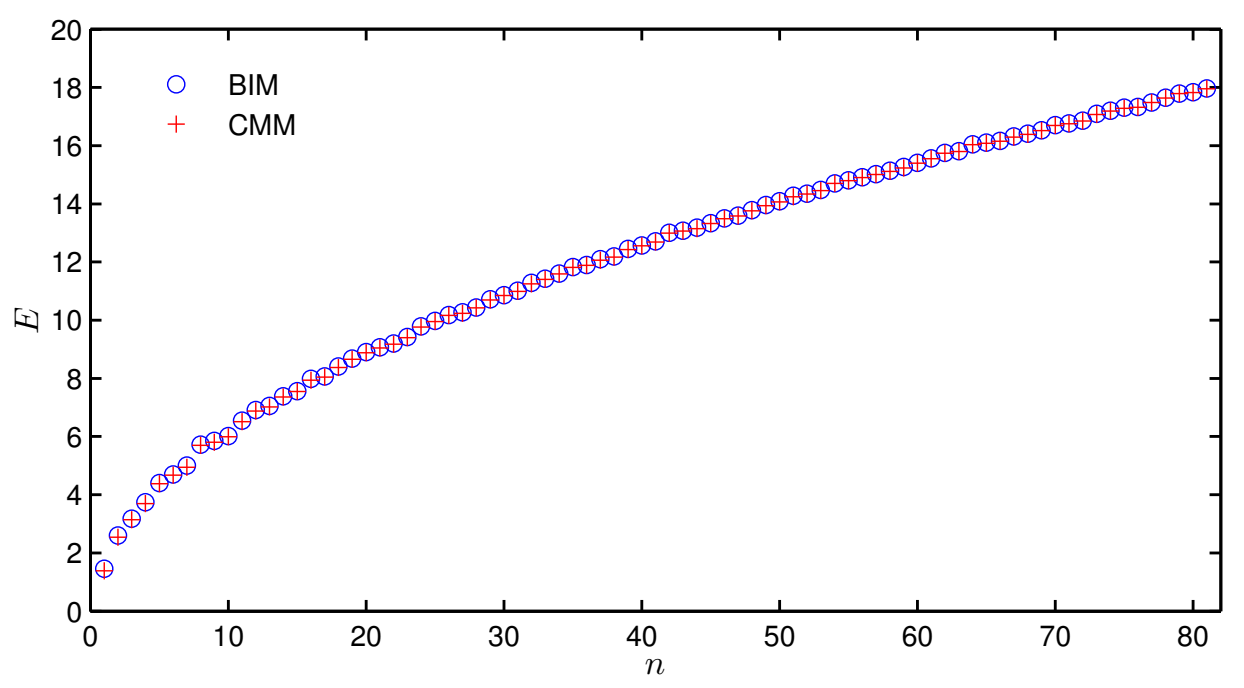

FIG. 8. Comparison of eigenenergies calculated from the conformal mapping method (CMM) and boundary-integral method (BIM). For a heart-shaped Dirac billiard whose classical dynamics are fully chaotic, the lowest 81 positive eigenenergy levels from both methods are shown.

can be expressed as [250]

$$
\psi(\boldsymbol{r})=\left(\begin{array}{c}
C_{1} Z_{l}(\mu r) \exp (i l \phi) \\
C_{2} Z_{l+1}(\mu r) \exp (i(l+1) \phi)
\end{array}\right),
$$

where $l=0, \pm 1, \pm 2, \ldots, \mu>0$, and $Z_{l}$ is the Bessel function. Substituting Eq. (49) into Eq. (48) and making use of the recursion relations of the Bessel functions: $Z_{l}^{\prime}(x) \pm l Z_{l}(x) / x= \pm Z_{l \mp 1}(x)$, one obtains

$$
\frac{C_{2}}{C_{1}}=i
$$

Since a physical solution must be finite at the origin, one has the eigenfunction

$$
\psi_{l m}(r, \phi)=N_{l m} \exp (i l \phi)\left(\begin{array}{c}
J_{l}\left(\mu_{l m} r\right) \\
i \exp (i \phi) J_{l+1}\left(\mu_{l m} r\right)
\end{array}\right)
$$

where the normalization factor is

$$
N_{l m}=\frac{1}{\sqrt{2 \pi \int_{0}^{1} \mathrm{~d} r r\left[J_{l}^{2}\left(\mu_{l m} r\right)+J_{l+1}^{2}\left(\mu_{l m} r\right)\right]}},
$$

and the circular domain is assumed to have unit radius for convenience: $r_{0}=1$.

The eigenvalues $\mu_{l m}$ can be determined from the boundary condition: $J_{l}\left(\mu_{l m}\right)=J_{l+1}\left(\mu_{l m}\right)$. The eigenfunctions satisfy

$$
\iint_{D} \mathrm{~d} \boldsymbol{r} \psi_{l^{\prime} m^{\prime}}^{\dagger}(\boldsymbol{r}) \psi_{l m}(\boldsymbol{r})=\delta_{l l^{\prime}} \delta_{m m^{\prime}}
$$

where, under the zero-flux boundary condition, the set $\left\{\psi_{l m}\right\}$ forms an orthonormal complete basis for the operator $-i \hat{\boldsymbol{\sigma}} \cdot \boldsymbol{\nabla}$ and its positive integral power $[-i \hat{\boldsymbol{\sigma}} \cdot \boldsymbol{\nabla}]^{n}(n=1,2, \ldots)$. For the circular domain, the analytic solutions of both the eigenvalues and eigenstates can be written as $\left\{\mu_{l m}, \psi_{l m}(r, \phi), l=0, \pm 1, \pm 2, \cdots, m=1,2, \cdots\right\}$. 


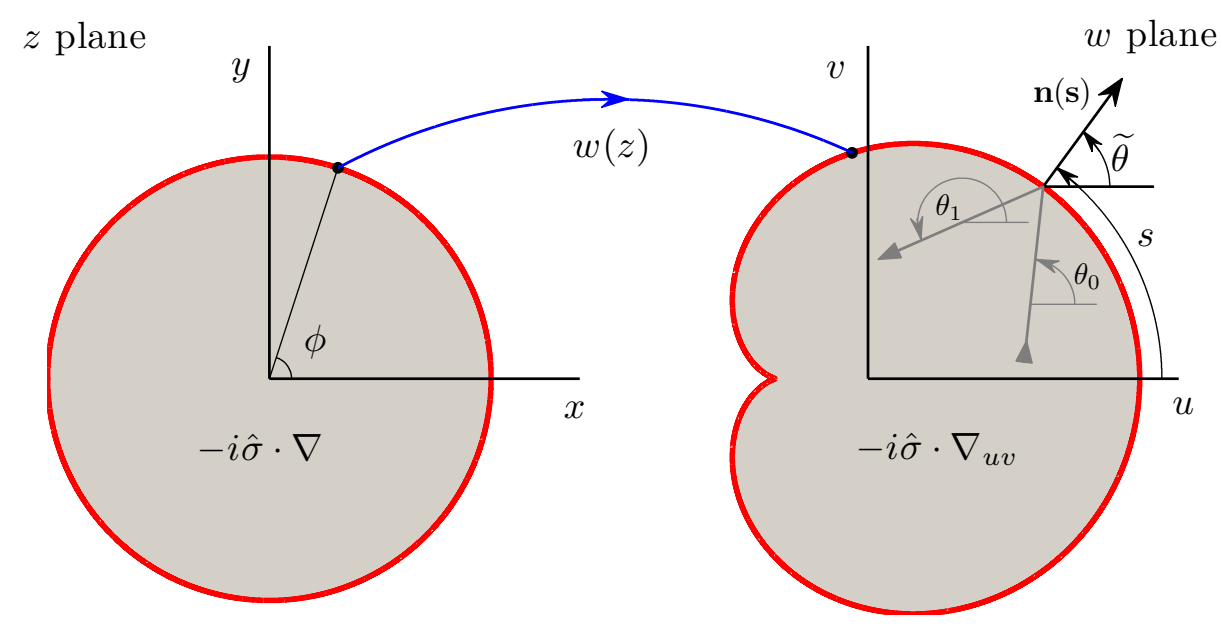

FIG. 9. Illustration of conformal mapping between a chaotic domain and a circle. Conformal transformation from the unit disc in $z=x+i y$ ( $z$-plane) to the billiard domain $D$ in $w=u+i v$ ( $w$-plane). The boundary is generated by the mapping function Eq. (54) with parameter $\beta=0.49$.

\section{Conformal mapping}

Given a closed domain with an analytic boundary, one seeks to identify a proper conformal mapping to transfer the domain into a circle. As shown in Fig. 9, an analytic billiard domain $D$ can be defined as a conformal transformation of the unit disc in the $w$-plane:

$$
u(x, y)+i v(x, y)=w(z) \equiv w\left(r e^{i \phi}\right), r \in[0,1],
$$

where $w(z)$ is an analytic function with non-vanishing derivative in $D$. For example, the heart shaped billiard shown in the right panel of Fig. 9 is given by:

$$
w(z)=\frac{1}{\sqrt{1+2 \beta^{2}}}\left(z+\beta z^{2}\right), \beta \in\left[0, \frac{1}{2}\right) .
$$

For $\beta=0.49$, the classical dynamics are chaotic [249].

The boundary can be parametrically defined as $u=\operatorname{Re}\left[w\left(e^{i \phi}\right)\right], v=\operatorname{Im}\left[w\left(e^{i \phi}\right)\right]$. The basic task is then to solve the following stationary Dirac equation:

$$
-i \hat{\boldsymbol{\sigma}} \cdot \nabla_{u v} \psi=k \psi
$$

together with the boundary condition $\psi_{2} /\left.\psi_{1}\right|_{\partial_{D}}=i e^{i \widetilde{\theta}}$, where $k \equiv E / \hbar c$. When being acted upon by the operator $-i \hat{\boldsymbol{\sigma}} \cdot \nabla_{u v}$, Eq. (55) becomes

$$
-\Delta_{u v} \mathbf{1} \psi=k^{2} \psi
$$

Using the conformal mapping $\Delta=|\mathrm{d} w / \mathrm{d} z|^{2} \Delta_{u v}$ to transform Eq. (56) to the unit disc in the $z$-plane, together with the definition $\psi^{\prime}(\boldsymbol{r})=\psi(u, v)$, one obtains the following form of Eq. (55) in the polar coordinates:

$$
\Delta \psi^{\prime}+k^{2} T(r, \phi) \psi^{\prime}=0
$$


where $T(r, \phi) \equiv|\mathrm{d} w / \mathrm{d} z|^{2}$.

To solve Eq. (57), one expands $\psi$ in terms of the eigenfunctions of the unit disc to obtain

$$
\psi^{\prime}(r, \phi)=\sum_{l=-\infty}^{\infty} \sum_{m=1}^{\infty} c_{l m} \psi_{l m}(r, \phi)
$$

where $c_{l m}$ are the expansion coefficients. Substituting Eq. (58) into Eq. (57) gives

$$
\frac{\nu_{l m}}{k^{2}}-\sum_{l^{\prime} m^{\prime}} M_{l m l^{\prime} m^{\prime}} \nu_{l^{\prime} m^{\prime}}=0
$$

where $\nu_{l m}=\mu_{l m} c_{l m}$, and

$$
\begin{aligned}
M_{l m l^{\prime} m^{\prime}}= & \frac{N_{l^{\prime} m^{\prime}} N_{l m}}{\mu_{l^{\prime} m^{\prime}} \mu_{l m}} \int_{0}^{2 \pi} \mathrm{d} \phi \exp \left\{i\left(l^{\prime}-l\right) \phi\right\} \int_{0}^{1} \mathrm{~d} r T(r, \phi) \\
& \left\{J_{l}\left(\mu_{l m} r\right) J_{l^{\prime}}\left(\mu_{l^{\prime} m^{\prime}} r\right)+J_{l+1}\left(\mu_{l m} r\right) J_{l^{\prime}+1}\left(\mu_{l^{\prime} m^{\prime}} r\right)\right\} .
\end{aligned}
$$

Once the eigenvalues $\lambda_{n}$ and the eigenvectors $\boldsymbol{\nu}^{(n)}$ of the matrix $\left(M_{l m l^{\prime} m^{\prime}}\right)$ have been obtained, one gets the complete solutions of Eq. (55) through the relations $k_{n}=1 / \sqrt{\lambda_{n}}$ and $c_{l m}^{(n)}=\nu_{l m}^{(n)} / \mu_{l m}$. A practical limitation is that, in actual computations, a truncated basis $\left\{\psi_{l m}(r, \phi)\right\}, l_{\min } \leq l \leq$ $l_{\max }, 1 \leq m \leq m_{\max }$ is used. Thus unusually high energy levels and the associated eigenstates cannot be determined accurately. Nonetheless, the conformal-mapping based method can yield a large number energy levels and the corresponding eigenstates with extremely high accuracy.

\section{G. Solutions of the Dirac equation in a ring domain subject to a central magnetic flux}

\section{Effects of a magnetic flux on the energy spectra}

It is useful to discuss the general properties of the relativistic quantum spectrum when a magnetic flux is present to gain insights into the solutions of the Dirac equation with a vector potential. Let $\alpha$ be a normalized parameter characterizing the magnetic flux in units of $\Phi_{0}$, and let $\left\{E_{j}(\alpha)\right\}$ denote the energy spectrum. Since the vector potential $\boldsymbol{A}$ depends on the gauge while the Dirac equation is gauge-invariant, one can choose a proper gauge transformation to eliminate $\boldsymbol{A}$ from the Dirac Hamiltonian in Eq. (11). An example of such transformation is

$$
\varphi(\boldsymbol{r}) \equiv\left(\begin{array}{c}
\varphi_{1} \\
\varphi_{2}
\end{array}\right)=\psi(\boldsymbol{r}) \times \exp \left[-i \frac{2 \pi}{\Phi_{0}} \int_{\boldsymbol{r}_{0}}^{\boldsymbol{r}} \boldsymbol{A}\left(\boldsymbol{r}^{\prime}\right) \cdot \mathrm{d} \boldsymbol{r}^{\prime}\right] .
$$

Substituting Eq. (61) into the Dirac equation and taking into account the boundary conditions, one obtains

$$
\hat{H}_{0} \varphi(\boldsymbol{r}) \equiv\left[-i \hbar c \hat{\boldsymbol{\sigma}} \cdot \boldsymbol{\nabla}+V(\boldsymbol{r}) \hat{\sigma}_{z}\right] \varphi(\boldsymbol{r})=E \varphi(\boldsymbol{r}),
$$

with the following complex boundary condition:

$$
\left.\frac{\varphi_{2}}{\varphi_{1}}\right|_{\partial \mathcal{B}}=\operatorname{sgn}(V) i \exp [i \tilde{\theta}(s)]
$$


A nontrivial point is, because $\psi$ is single valued, $\varphi$ must be multivalued. As a result, for any circuit $C$ about the flux line, $\varphi$ will acquire a phase factor:

$$
\hat{c} \varphi=\exp \left[-i \frac{2 \pi}{\Phi_{0}} \oint_{C} \boldsymbol{A} \cdot \mathrm{d} \boldsymbol{r}\right] \varphi=\exp \left(-2 \pi i W_{C} \alpha\right) \varphi,
$$

where the operator $\hat{c}$ denotes the circuit operation acting on $\varphi$ and $W_{C}(= \pm 1, \pm 2, \cdots)$ is the winding number of $C$. From Eqs. (62)-(64), one can conclude that, because the complex boundary condition does not depend on $\alpha$, it is not possible to generate the so-called "false time reversal symmetry breaking" only by tuning the quantum flux parameter $\alpha$, in contrast to the nonrelativistic counterpart [93]. If the boundary $\mathcal{B}$ is chosen to be geometrically asymmetric and the dynamics are chaotic in the classical limit, the relativistic quantum spectral statistics are characterized by those of the Gaussian unitary ensemble (GUE), regardless of the value of $\alpha$. Furthermore, the spectrum $\left\{E_{j}(\alpha)\right\}$ does not possess the symmetry with respect to flux reversal $(\alpha \rightarrow-\alpha)$, in contrast to the nonrelativistic quantum spectra as determined by the Schrödinger equation where the symmetry is preserved. This can be shown explicitly, as follows.

For the Dirac ring system, the flux-reversal operator $\hat{f}$ can be represented as

$$
\hat{f}=\hat{U} \hat{K}
$$

where $\hat{U}$ is unitary and commutes with $\hat{c}$, and $\hat{K}$ denotes complex conjugate:

$$
[\hat{f}, \hat{c}]=0 .
$$

It can be seen that $\varphi$ transforms to

$$
\varphi^{\prime} \equiv\left[\varphi_{1}^{\prime}, \varphi_{2}^{\prime}\right]^{\mathrm{T}}=\hat{f} \varphi=\hat{U} \varphi^{*}
$$

and

$$
\hat{c} \varphi^{\prime}=\exp \left[-2 \pi i W_{C}(-\alpha)\right] \varphi^{\prime} .
$$

In order for $E_{j}(\alpha)=E_{j}(-\alpha)$ to hold, $\hat{f}$ must satisfy

$$
\left[\hat{f}, \hat{H}_{0}\right]=0
$$

and $\varphi^{\prime}$ must follow the same boundary condition as in Eq. (63). It was pointed out by Berry and Mondragon [93] that, if $V(\boldsymbol{r})$ possesses no geometric symmetry, no operator would exist satisfying Eq. (65) and Eq. (68) simultaneously. However, it may be possible to find an operator with the form given by Eq. (65), which commutes with $\hat{H}_{0}$ if $V(\boldsymbol{r})$ does possess any geometric symmetry. In the ring domain, any such possible operator would not satisfy the pre-condition Eq. (66) at the same time. Consequently, the energy spectrum $\left\{E_{j}(\alpha)\right\}$ does not possess the flux-reversal symmetry, i.e., $E_{j}(\alpha) \neq E_{j}(-\alpha)$.

By considering the specific situation of $\hat{U}=i \hat{\sigma}_{y}$, one can see that the flux-reversal symmetry emerges if the sign of the potential $V$ is changed simultaneously: $\{\alpha \rightarrow-\alpha, V \rightarrow-V\}$, which is the primary reason that the energy spectrum in graphene is symmetric with respect to $\alpha=0$ when both valleys are included [251]. From Eqs. (62)-(64), one gets

$$
E_{j}(\alpha)=E_{j}(\alpha+1) .
$$

Studying the relativistic quantum spectrum for a billiard for the flux parameter in the range $-1 / 2 \leq \alpha \leq 1 / 2$ thus suffices, which corresponds to the first "Brillouin zone." 


\section{Dirac billiard with a singular Aharonov-Bohm (AB) magnetic flux}

The Dirac AB billiard consists of a single massless spin-half particle of charge $q$ confined by hard walls (infinite mass confinement) and is threaded by a single line of magnetic flux $\Phi$ at the origin, which is a paradigmatic setting for studying persistent currents in chaotic Dirac systems (Chap. VII). The position of the flux line is a singular point, which can be excluded by assuming an inner disk of infinite mass potential with a vanishing inner radius centered at this point. The flux will introduce a modulating phase and, because of its point-like nature, it has little effect on the spinor wavefunctions. In fact, in quantum field theory there are similar models but with a focus on the vacuum energy caused by the $\mathrm{AB}$ flux, with a different setup of the boundary condition $[252,253]$. To obtain the eigensolutions of the chaotic Dirac AB billiard, one can exploit the conformal mapping method based on the eigenstates of the circular Dirac AB billiard.

Analytic solution of the circular Dirac AB billiard. For a circular ring domain of inner radius $\xi \rightarrow 0$ threaded by a single line of magnetic flux $\Phi$ at the origin, one can choose a non-divergent gauge in which the lines of the vector potential $\boldsymbol{A}$ are the contours of a scalar function $F(\boldsymbol{r})=$ $-\ln (|\boldsymbol{r}|)$,

$$
\boldsymbol{A}(\boldsymbol{r})=\frac{\Phi}{2 \pi}\left(\frac{\partial F}{\partial y},-\frac{\partial F}{\partial x}\right)=\frac{\Phi}{2 \pi}\left(-\frac{\sin \phi}{r}, \frac{\cos \phi}{r}\right),
$$

where $\nabla \cdot \boldsymbol{A}=0, \nabla \times \boldsymbol{A}=\hat{\boldsymbol{z}} \Phi \delta(\boldsymbol{r})$, and $\hat{\boldsymbol{z}}$ is the unit vector normal to the $(x, y)$-plane. The Dirac equation in the presence of an $\mathrm{AB}$ flux is

$$
c \hat{\boldsymbol{\sigma}} \cdot(-i \hbar \boldsymbol{\nabla}+e \boldsymbol{A}) \psi=E \psi,
$$

where $\psi=\left[\psi_{1}, \psi_{2}\right]^{T}$ with the boundary condition $\psi_{2} /\left.\psi_{1}\right|_{\partial_{D}}=i e^{i \tilde{\theta}(s)}$, where $s$ is the arc length of the boundary, starting from the cross point of the boundary with the positive $x$-axis, and $\widetilde{\theta}(s)$ is the angle to the positive $x$-axis for the normal vector at $s$. For a circularly symmetric ring boundary, one has

$$
\left[\hat{J}_{z}, \hat{H}\right]=0,
$$

where $\hat{J}_{z}=-i \hbar \partial_{\phi}+(\hbar / 2) \hat{\sigma}_{z}$ is the total angular momentum operator. The simultaneous eigenstates of $\hat{H}$ and $\hat{J}_{z}$ are

$$
\hat{J}_{z} \psi=(l+1 / 2) \hbar \psi .
$$

The general form of the solutions of (71) is

$$
\psi(\boldsymbol{r})=N\left(\begin{array}{c}
\chi_{1}(r) \\
i \chi_{2}(r) e^{i \phi}
\end{array}\right) e^{i l \phi},
$$

where $l=0, \pm 1, \pm 2, \cdots$ and $N$ is the normalization factor. The Dirac equation in the polar coordinates is

$$
\left(\begin{array}{cc}
0 & e^{-i \theta}\left(\frac{\partial}{\partial r}-\frac{i}{r} \frac{\partial}{\partial \theta}-\frac{\alpha}{r}\right) \\
e^{i \theta}\left(\frac{\partial}{\partial r}+\frac{i}{r} \frac{\partial}{\partial \theta}+\frac{\alpha}{r}\right) & 0
\end{array}\right) \psi(\boldsymbol{r})=i \mu \psi(\boldsymbol{r}),
$$

where $\mu \equiv E /(\hbar c)$ and $\alpha \equiv \Phi / \Phi_{0}$. Substituting Eq. (72) into Eq. (73), one gets

$$
\left(\begin{array}{cc}
-\mu & \frac{\mathrm{d}}{\mathrm{d} r}+\frac{l+1-\alpha}{r} \\
-\frac{\mathrm{d}}{\mathrm{d} r}+\frac{l-\alpha}{r} & -\mu
\end{array}\right)\left(\begin{array}{l}
\chi_{1}(r) \\
\chi_{2}(r)
\end{array}\right)=0 .
$$


Canceling $\chi_{2}$ in Eq. (74), one gets the Bessel's differential equation

$$
\left(\frac{\mathrm{d}^{2}}{\mathrm{~d} R^{2}}+\frac{1}{R} \frac{\mathrm{d}}{\mathrm{d} R}+1-\frac{(l-\alpha)^{2}}{R^{2}}\right) \chi_{1}(r)=0
$$

where $R=\mu r, \nu=l-\alpha$, and $\chi_{1}(r)$ can be written as a linear combination of the Bessel function of the first kind, $J_{\nu}(R)$, and the Bessel function of the second kind, $Y_{\nu}(R)$, as

$$
\chi_{1}(R)=J_{\nu}(R)+\beta Y_{\nu}(R),
$$

where $\beta$ is a coefficient and can be determined by the boundary conditions, The second spinor component $\chi_{2}(R)$ satisfies the equation

$$
-R \chi_{2}(R)=R \chi_{1}^{\prime}(R)-\nu \chi_{1}(R)
$$

Application of the recursive relation of Bessel functions leads to

$$
\chi_{2}(R)=J_{\nu+1}(R)+\beta Y_{\nu+1}(R) .
$$

Imposing the inner and outer boundary conditions, one gets

$$
\left\{\begin{array}{l}
J_{\nu}(\mu \xi)+\beta Y_{\nu}(\mu \xi)=-\left(J_{\nu+1}(\mu \xi)+\beta Y_{\nu+1}(\mu \xi)\right) \\
J_{\nu}(\mu)+\beta Y_{\nu}(\mu)=J_{\nu+1}(\mu)+\beta Y_{\nu+1}(\mu)
\end{array}\right.
$$

solutions of which gives the following expression for $\beta$ :

$$
\beta=-\frac{J_{\nu+1}(\mu \xi)+J_{\nu}(\mu \xi)}{Y_{\nu+1}(\mu \xi)+Y_{\nu}(\mu \xi)}=-\frac{J_{\nu+1}(\mu)-J_{\nu}(\mu)}{Y_{\nu+1}(\mu)-Y_{\nu}(\mu)},
$$

where the eigenvalue $\mu$ (and hence $E=\hbar c \mu$ ) can be obtained by solving

$$
\left[J_{\nu+1}(\mu)-J_{\nu}(\mu)\right] \cdot\left[Y_{\nu+1}(\mu \xi)+Y_{\nu}(\mu \xi)\right]=\left[J_{\nu+1}(\mu \xi)+J_{\nu}(\mu \xi)\right] \cdot\left[Y_{\nu+1}(\mu)-Y_{\nu}(\mu)\right]
$$

Equation (80) can be simplified by exploiting the special properties of the Bessel functions:

$$
\begin{gathered}
\lim _{x \rightarrow 0} J_{\nu}(x) \sim \frac{x^{\nu}}{2^{\nu} \Gamma(1+\nu)}= \begin{cases}0 & (\nu>0 \text { or } \nu=- \text { integer }), \\
1 & (\nu=0), \\
\infty & (\nu<0 \text { and } \nu \neq- \text { integer }),\end{cases} \\
Y_{\nu}(x)=\frac{\cos (\nu \pi) J_{\nu}(x)-J_{-\nu}(x)}{\sin (\nu \pi)}, \quad \lim _{x \rightarrow 0} Y_{\nu}(x) \sim \infty .
\end{gathered}
$$

There are distinct cases.

A. For $\nu$ being an integer, the right hand side of Eq. (80) is finite. Since both $Y_{\nu+1}(\mu \xi)$ and $Y_{\nu}(\mu \xi)$ diverge as $\xi$ goes to zero, $\left(J_{\nu+1}(\mu)-J_{\nu}(\mu)\right)$ must be zero. One thus has

Case 1: $\nu$ is an integer, $\xi \rightarrow 0$. Equation (80) becomes

$$
J_{\nu}(\mu) \approx J_{\nu+1}(\mu) .
$$

B. For $\nu$ not being an integer, $Y_{\nu}$ can be expressed as a linear combination of $J_{\nu}$ and $J_{-\nu}$, so Eq. (80) can be simplified as

$$
\begin{aligned}
& {\left[J_{\nu+1}(\mu)-J_{\nu}(\mu)\right] \cdot\left[J_{-(\nu+1)}(\mu \xi)-J_{-\nu}(\mu \xi)\right] } \\
= & {\left[J_{\nu+1}(\mu \xi)+J_{\nu}(\mu \xi)\right] \cdot\left[J_{-(\nu+1)}(\mu)+J_{-\nu}(\mu)\right] . }
\end{aligned}
$$


Case 2: $\nu$ is not an integer, $\nu>0$ and $\xi \rightarrow 0 . \quad$ In this case, one has $J_{-(\nu+1)}(\mu \xi)-J_{-\nu}(\mu \xi) \rightarrow$ $\infty$ and $J_{\nu+1}(\mu \xi)+J_{\nu}(\mu \xi) \rightarrow 0$, which lead to

$$
J_{\nu}(\mu) \approx J_{\nu+1}(\mu)
$$

Case 3: $\nu$ is not an integer, $\nu<-1$ and $\xi \rightarrow 0 . \quad$ In this case, one has $J_{-(\nu+1)}(\mu \xi)-J_{-\nu}(\mu \xi) \rightarrow$ 0 and $J_{\nu+1}(\mu \xi)+J_{\nu}(\mu \xi) \rightarrow \infty$, which give

$$
J_{-\nu}(\mu) \approx-J_{-(\nu+1)}(\mu)
$$

C. For $-1<\nu<0$, one has $J_{-(\nu+1)}(\mu \xi) \sim \xi^{-(1+\nu)} / \Gamma(-\nu), J_{\nu}(\mu \xi) \sim \xi^{\nu} / \Gamma(1+\nu)$, and $J_{-\nu}(\mu \xi) \rightarrow 0, J_{\nu+1}(\mu \xi) \rightarrow 0$. There are three subcases.

Case 4: $-1 / 2<\nu<0$ and $\xi \rightarrow 0$. In this case, one has $J_{\nu+1}(\mu \xi) / J_{\nu}(\mu \xi) \sim \xi^{-1-2 \nu} \Gamma(1+$ $\nu) / \Gamma(-\nu) \rightarrow+\infty$ and, hence,

$$
J_{\nu}(\mu) \approx J_{\nu+1}(\mu)
$$

Case 5: $-1<\nu<-1 / 2$ and $\xi \rightarrow 0$. One has $J_{-(\nu+1)}(\mu \xi) / J_{\nu}(\mu \xi) \sim \xi^{-1-2 \nu} \Gamma(1+$ $\nu) / \Gamma(-\nu) \rightarrow 0$ and thus

$$
J_{-\nu}(\mu) \approx-J_{-(\nu+1)}(\mu)
$$

Case 6: $\nu=-1 / 2$ and $\xi \rightarrow 0 . \quad$ One has $Y_{-\frac{1}{2}}(x)=J_{\frac{1}{2}}(x)$ and $Y_{\frac{1}{2}}(x)=-J_{-\frac{1}{2}}(x)$. Using Eq. (79), one gets $\beta=1$ and $J_{\nu+1}(\mu)-J_{\nu}(\mu)=J_{\nu+1}(\mu)+J_{\nu}(\mu)$, which give

$$
J_{-\frac{1}{2}}(\mu) \approx 0
$$

For the simplified equations (81)-(86), one can get the eigenvalues $\mu_{l m}(\alpha)$, where the magnetic flux $\alpha$ can be regarded as a control parameter, the quantities $l$ and $\nu$ are related to each other by $\nu=l-\alpha$, and $m$ represents the $m$ 'th solution for a given $l$.

Once the function $\mu_{l m}(\alpha)$ has been found, one can substitute it back into Eq. (79) to get the corresponding quantity $\beta_{l m}(\alpha)$. Substituting these two quantities back to Eqs. (72), (76), and (77), one obtains the corresponding eigenfunction $\psi_{l m}(\alpha)$ :

$$
\begin{aligned}
\psi_{l m}(\boldsymbol{r}, \alpha) & =N_{l m}\left(\begin{array}{c}
\chi_{1, l m}(r) \\
i \chi_{2, l m}(r) e^{i \theta}
\end{array}\right) e^{i l \theta} \\
& =N_{l m}\left(\begin{array}{c}
J_{\nu}\left(\mu_{l m} r\right)+\beta_{l m} Y_{\nu}\left(\mu_{l m} r\right) \\
i\left(J_{\nu+1}\left(\mu_{l m} r\right)+\beta_{l m} Y_{\nu+1}\left(\mu_{l m} r\right)\right) e^{i \theta}
\end{array}\right) e^{i l \theta} .
\end{aligned}
$$

Simplified expressions for the eigenfunctions can be obtained through appropriate approximations for different cases.

A. When $\nu$ is an integer, the divergence property of $Y_{\nu}(x)$ is

$$
\begin{aligned}
\lim _{x \rightarrow 0} Y_{0}(x) & \left.\sim \frac{2}{\pi} \ln \frac{x}{2}\right|_{x \rightarrow 0} \text { and } \\
\lim _{x \rightarrow 0} Y_{\nu}(x) & \left.\sim \frac{-(\nu-1) !}{\pi}\left(\frac{x}{2}\right)^{-\nu}\right|_{x \rightarrow 0} \quad \nu=1,2, \cdots, \\
Y_{-\nu}(x) & =(-1)^{\nu} Y_{\nu}(x)
\end{aligned}
$$


Case 1a: $\nu$ is a non-negative integer and $r \geq \xi$. One has

$$
\beta \approx-\frac{J_{\nu}(\mu \xi)}{Y_{\nu+1}(\mu \xi)}
$$

and

$$
\beta Y_{\nu}(\mu r)=-\frac{J_{\nu}(\mu \xi)}{Y_{\nu+1}(\mu \xi)} Y_{\nu}(\mu r) \approx 0
$$

as $Y_{\nu+1}$ diverges faster than $Y_{\nu}$ at $r=\xi$. One also has

$$
\beta Y_{\nu+1}(\mu r)=-\frac{J_{\nu}(\mu \xi)}{Y_{\nu+1}(\mu \xi)} Y_{\nu+1}(\mu r)
$$

and

$$
\left\{\begin{array}{l}
\chi_{1, l m}(r)=J_{\nu}\left(\mu_{l m} r\right), \\
\chi_{2, l m}(r)=J_{\nu+1}\left(\mu_{l m} r\right)-\frac{J_{\nu}\left(\mu_{l m} \xi\right)}{Y_{\nu+1}\left(\mu_{l m} \xi\right)} Y_{\nu+1}\left(\mu_{l m} r\right) .
\end{array}\right.
$$

Note that the term $-J_{\nu}\left(\mu_{l m} \xi\right) Y_{\nu+1}\left(\mu_{l m} r\right) / Y_{\nu+1}\left(\mu_{l m} \xi\right)$ has little influence on the eigenvalues and the eigenfunctions. The reason is that, for $r \gg \xi, Y_{\nu+1}\left(\mu_{l m} r\right)$ is finite, while

$$
J_{\nu}\left(\mu_{l m} \xi\right) / Y_{\nu+1}\left(\mu_{l m} \xi\right) \sim-\left[\pi /\left(2^{\nu+1 / 2} \nu !\right)^{2}\right]\left(\mu_{l m} \xi\right)^{2 \nu+1} \sim-\xi^{2 \nu+1} \sim 0 .
$$

As a result, the whole term approaches zero. For $r=\xi$, this term becomes

$$
-J_{\nu}\left(\mu_{l m} \xi\right) \sim-\left(\mu_{l m} \xi\right)^{\nu} /\left(2^{\nu} \nu !\right)
$$

and is finite. However, this term guarantees the boundary condition

$$
\chi_{2, l m}\left(\mu_{l m} \xi\right) / \chi_{1, l m}\left(\mu_{l m} \xi\right)=-1
$$

at the inner boundary $r=\xi$ and leads to a clockwise flow.

Case 1b: $\nu$ is a negative integer and $r \geq \xi$. One has

$$
\begin{aligned}
\beta & \approx-\frac{J_{\nu+1}(\mu \xi)}{Y_{\nu}(\mu \xi)}, \\
\beta Y_{\nu}(\mu r) & =-\frac{J_{\nu+1}(\mu \xi)}{Y_{\nu}(\mu \xi)} Y_{\nu}(\mu r), \\
\beta Y_{\nu+1}(\mu r) & =-\frac{J_{\nu+1}(\mu \xi)}{Y_{\nu}(\mu \xi)} Y_{\nu+1}(\mu r) \approx 0
\end{aligned}
$$

and, hence,

$$
\left\{\begin{array}{l}
\chi_{1, l m}(r)=J_{\nu}\left(\mu_{l m} r\right)-\frac{J_{\nu+1}\left(\mu_{l m} \xi\right)}{Y_{\nu}\left(\mu_{l m} \xi\right)} Y_{\nu}\left(\mu_{l m} r\right) \\
\chi_{2, l m}(r)=J_{\nu+1}\left(\mu_{l m} r\right) .
\end{array}\right.
$$

Similarly, the term $-J_{\nu+1}\left(\mu_{l m} \xi\right) Y_{\nu}\left(\mu_{l m} r\right) / Y_{\nu}\left(\mu_{l m} \xi\right)$ has little effect on the eigenvalues and eigenfunctions but guarantees the boundary condition at the inner circle. Note that, if $\nu$ is an integer, one has $J_{\nu}\left(\mu_{l m} r\right)=(-1)^{\nu} J_{-\nu}\left(\mu_{l m} r\right)$, and further simplification can be obtained.

B. For $\nu$ not being an integer, the asymptotic behavior of the first class Bessel Function is

$$
\left.\lim _{x \rightarrow 0} J_{\nu}(x) \sim \frac{x^{\nu}}{2^{\nu} \Gamma(1+\nu)}\right|_{x \rightarrow 0}
$$

This provides the base to treat several cases. 
Case 2: $\nu$ is not an integer and $\nu>0 . \quad$ In this case, one has

$$
\begin{aligned}
Y_{\nu}(\mu \xi) & \approx-\frac{1}{\sin \nu \pi} \cdot J_{-\nu}(\mu \xi) \\
Y_{\nu+1}(\mu \xi) & \approx \frac{1}{\sin \nu \pi} \cdot J_{-(\nu+1)}(\mu \xi) \\
\beta & =-\frac{\sin \nu \pi \cdot\left[J_{\nu+1}(\mu \xi)+J_{\nu}(\mu \xi)\right]}{J_{-(\nu+1)}(\mu \xi)-J_{-\nu}(\mu \xi)} \approx-\frac{\sin \nu \pi \cdot J_{\nu}(\mu \xi)}{J_{-(\nu+1)}(\mu \xi)}, \\
\beta \cdot Y_{\nu}(\mu r) & \approx \frac{J_{\nu}(\mu \xi)}{J_{-(\nu+1)}(\mu \xi)} \cdot J_{-\nu}(\mu r) \approx 0 \\
\beta \cdot Y_{\nu+1}(\mu r) & \approx-\frac{J_{\nu}(\mu \xi)}{J_{-(\nu+1)}(\mu \xi)} \cdot J_{-(\nu+1)}(\mu r)
\end{aligned}
$$

and, hence,

$$
\left\{\begin{array}{l}
\chi_{1, l m}(r) \approx J_{\nu}\left(\mu_{l m} r\right) \\
\chi_{2, l m}(r) \approx J_{\nu+1}\left(\mu_{l m} r\right)-\frac{J_{\nu}\left(\mu_{l m} \xi\right)}{J_{-(\nu+1)}\left(\mu_{l m} \xi\right)} \cdot J_{-(\nu+1)}\left(\mu_{l m} r\right) .
\end{array}\right.
$$

For $r>\xi$, the second term in $\chi_{2, l m}(r)$ approaches zero, so $\chi_{2, l m}(r) \approx J_{\nu+1}\left(\mu_{l m} r\right)$. While for $r \rightarrow \xi \rightarrow 0$, one has $\chi_{2, l m}(r) \approx-J_{\nu}\left(\mu_{l m} \xi\right)$, which satisfies the boundary condition (63) at $r=\xi$ and leads to a clockwise current at the inner boundary.

Case 3a: $\nu$ is not an integer, $\nu<-1$ and $\nu$ is not a half-integer. In this case, one has

$$
\begin{aligned}
\beta & \approx-\tan \nu \pi\left(1-\frac{1}{\cos \nu \pi} \cdot \frac{J_{-(\nu+1)}(\mu \xi)}{J_{\nu}(\mu \xi)}\right), \\
\beta \cdot Y_{\nu}(\mu r) & \approx-J_{\nu}(\mu r)+\frac{1}{\cos \nu \pi} \cdot J_{-\nu}(\mu r)+\frac{1}{\cos \nu \pi} \frac{J_{-(\nu+1)}(\mu \xi)}{J_{\nu}(\mu \xi)} \cdot J_{\nu}(\mu r), \\
\beta \cdot Y_{\nu+1}(\mu r) & =-J_{\nu+1}(\mu r)-\frac{1}{\cos \nu \pi} \cdot J_{-(\nu+1)}(\mu r)
\end{aligned}
$$

and, hence,

$$
\left\{\begin{array}{l}
\chi_{1, l m}(r) \approx J_{-\nu}\left(\mu_{l m} r\right)+\frac{J_{-(\nu+1)}\left(\mu_{l m} \xi\right)}{J_{\nu}\left(\mu_{l m} \xi\right)} J_{\nu}\left(\mu_{l m} r\right) \\
\chi_{2, l m}(r) \approx-J_{-(\nu+1)}\left(\mu_{l m} r\right) .
\end{array}\right.
$$

Case 3b: $\nu$ is not an integer, $\nu<-1$ and $\nu$ is a half-integer. One has

$$
\begin{aligned}
Y_{\nu}(\mu \xi) & \approx-\frac{1}{\sin \nu \pi} \cdot J_{-\nu}(\mu \xi), \\
Y_{\nu+1}(\mu \xi) & \approx \frac{1}{\sin \nu \pi} \cdot J_{-(\nu+1)}(\mu \xi), \\
\beta & =-\frac{\sin \nu \pi \cdot\left[J_{\nu+1}(\mu \xi)+J_{\nu}(\mu \xi)\right]}{J_{-(\nu+1)}(\mu \xi)-J_{-\nu}(\mu \xi)} \approx-\frac{\sin \nu \pi \cdot J_{\nu}(\mu \xi)}{J_{-(\nu+1)}(\mu \xi)}, \\
\beta \cdot Y_{\nu}(\mu r) & \approx \frac{J_{\nu}(\mu \xi)}{J_{-(\nu+1)}(\mu \xi)} \cdot J_{-\nu}(\mu r), \\
\beta \cdot Y_{\nu+1}(\mu r) & \approx-\frac{J_{\nu}(\mu \xi)}{J_{-(\nu+1)}(\mu \xi)} \cdot J_{-(\nu+1)}(\mu r) .
\end{aligned}
$$


Combining the term $J_{\nu}(\mu \xi) / J_{-(\nu+1)}(\mu \xi)$ into the normalization factor leads to the same formula as Eq. (91)

$$
\left\{\begin{array}{l}
\chi_{1, l m}(r) \approx J_{-\nu}\left(\mu_{l m} r\right)+\frac{J_{-(\nu+1)}\left(\mu_{l m} \xi\right)}{J_{\nu}\left(\mu_{l m} \xi\right)} J_{\nu}\left(\mu_{l m} r\right) \\
\chi_{2, l m}(r) \approx-J_{-(\nu+1)}\left(\mu_{l m} r\right) .
\end{array}\right.
$$

Case 4: $-1 / 2<\nu<0 . \quad$ In this case, one has

$$
\begin{aligned}
Y_{\nu}(\mu \xi) & =\frac{\cos \nu \pi}{\sin \nu \pi} \cdot J_{\nu}(\mu \xi)-\frac{1}{\sin \nu \pi} \cdot J_{-\nu}(\mu \xi) \approx \frac{\cos \nu \pi}{\sin \nu \pi} \cdot J_{\nu}(\mu \xi), \\
Y_{\nu+1}(\mu \xi) & =\frac{\cos (\nu+1) \pi}{\sin (\nu+1) \pi} \cdot J_{\nu+1}(\mu \xi)-\frac{1}{\sin (\nu+1) \pi} \cdot J_{-(\nu+1)}(\nu \xi) \approx \frac{1}{\sin \nu \pi} \cdot J_{-(\nu+1)}(\mu \xi), \\
\beta & =-\frac{J_{\nu+1}(\mu \xi)+J_{\nu}(\mu \xi)}{\left[\sin \nu \pi \cdot J_{\nu}(\mu \xi)\right.}
\end{aligned}
$$

and

$$
\begin{aligned}
\beta \cdot Y_{\nu}(\mu r) & =-\frac{J_{\nu}(\mu \xi)}{J_{-(\nu+1)}(\mu \xi)} \cdot\left[\cos \nu \pi \cdot J_{\nu}(\mu r)-J_{-\nu}(\mu r)\right] \approx 0, \\
\beta \cdot Y_{\nu+1}(\mu r) & \approx-\frac{J_{\nu}(\mu \xi)}{J_{-(\nu+1)}(\mu \xi)} \cdot J_{-(\nu+1)}(\mu r) .
\end{aligned}
$$

One thus obtains

$$
\left\{\begin{array}{l}
\chi_{1, l m}(r) \approx J_{\nu}\left(\mu_{l m} r\right)+\frac{J_{\nu}\left(\mu_{l m} \xi\right)}{J_{-(\nu+1)}\left(\mu_{l m} \xi\right)} \cdot J_{-\nu}\left(\mu_{l m} r\right) \\
\chi_{2, l m}(r) \approx J_{\nu+1}\left(\mu_{l m} r\right)-\frac{J_{\nu}(\mu \xi)}{J_{-(\nu+1)}\left(\mu_{l m} \xi\right)} \cdot J_{-(\nu+1)}\left(\mu_{l m} r\right) .
\end{array}\right.
$$

Case 5: $-1<\nu<-1 / 2 . \quad$ In this case, one has

$$
\begin{aligned}
Y_{\nu}(\mu \xi) & \approx \frac{\cos \nu \pi}{\sin \nu \pi} \cdot J_{\nu}(\mu \xi) \\
Y_{\nu+1}(\mu \xi) & \approx \frac{1}{\sin \nu \pi} \cdot J_{-(\nu+1)}(\mu \xi), \\
\beta & \approx-\tan \nu \pi\left(1-\frac{1}{\cos \nu \pi} \cdot \frac{J_{-(\nu+1)}(\mu \xi)}{J_{\nu}(\mu \xi)}\right), \\
\beta \cdot Y_{\nu}(\mu r) & \approx-J_{\nu}(\mu r)+\frac{1}{\cos \nu \pi} \cdot\left[J_{-\nu}(\mu r)+\frac{J_{-(\nu+1)}(\mu r)}{J_{\nu}(\mu \xi)} \cdot J_{\nu}(\mu r)\right], \\
\beta \cdot Y_{\nu+1}(\mu r) & =-J_{\nu+1}(\mu \xi)-\frac{1}{\cos \nu \pi} \cdot\left(J_{-(\nu+1)}(\mu r)+\frac{J_{-(\nu+1)}(\mu \xi)}{J_{\nu}(\mu \xi)} \cdot J_{\nu+1}(\mu r)\right)
\end{aligned}
$$

and, hence,

$$
\left\{\begin{array}{l}
\chi_{1, l m}(r)=\frac{J_{-(\nu+1)}\left(\mu_{l m} \xi\right)}{J_{\nu}\left(\mu_{l m} \xi\right)} \cdot J_{\nu}\left(\mu_{l m} r\right)+J_{-\nu}\left(\mu_{l m} r\right) \\
\chi_{2, l m}(r)=-J_{-(\nu+1)}\left(\mu_{l m} r\right)+\frac{J_{-(\nu+1)}\left(\mu_{l m} \xi\right)}{J_{\nu}\left(\mu_{l m} \xi\right)} \cdot J_{\nu+1}\left(\mu_{l m} r\right)
\end{array}\right.
$$




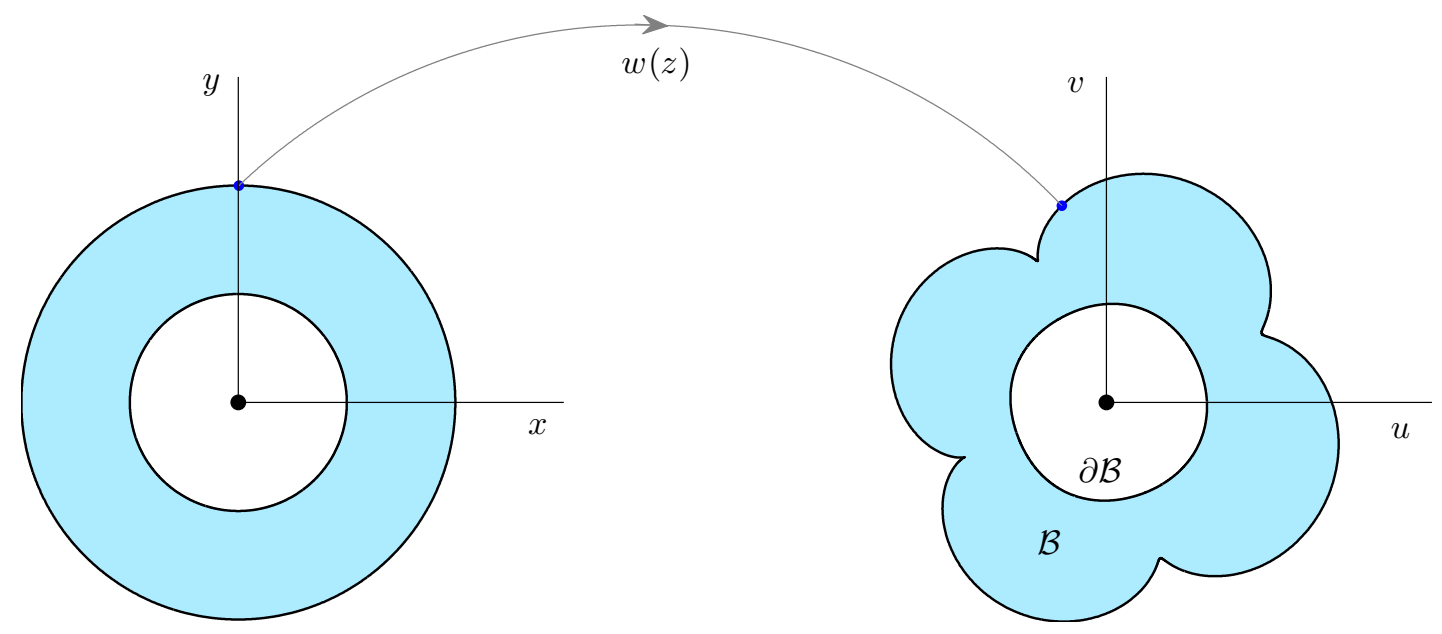

FIG. 10. Conformal transform for a ring domain. Conformal mapping from the unit disc in $z=x+i y$ ( $z$-plane) to a ring billiard domain in $w=u+i v$ ( $w$-plane).

Case 6: $\nu=-1 / 2 . \quad$ In this case, one has $\beta \approx 1$ and, hence,

$$
\left\{\begin{array}{l}
\chi_{1, l m}(r) \approx J_{-1 / 2}\left(\mu_{l m} r\right)+J_{1 / 2}\left(\mu_{l m} r\right), \\
\chi_{2, l m}(r) \approx J_{1 / 2}\left(\mu_{l m} r\right)-J_{-1 / 2}\left(\mu_{l m} r\right) .
\end{array}\right.
$$

Substituting Eqs. (88)-(95) into Eq. (87), one gets the simplified eigenfunctions $\psi_{l m}(\boldsymbol{r}, \alpha)$ associated with the eigenvalues $\mu_{l m}(\alpha)$.

In the numerical computation of the eigenfunctions, the small value terms can be ignored and the following approximations can be used:

$$
\begin{gathered}
\left\{\begin{array}{l}
\chi_{1, l m}(r)=J_{\nu}\left(\mu_{l m} r\right), \\
\chi_{2, l m}(r)=J_{\nu+1}\left(\mu_{l m} r\right), \text { for } \nu>-1 / 2,
\end{array}\right. \\
\left\{\begin{array}{l}
\chi_{1, l m}(r) \approx J_{-\nu}\left(\mu_{l m} r\right), \\
\chi_{2, l m}(r) \approx-J_{-(\nu+1)}\left(\mu_{l m} r\right), \text { for } \nu<-1 / 2,
\end{array}\right. \\
\left\{\begin{array}{l}
\chi_{1, l m}(r) \approx J_{-1 / 2}\left(\mu_{l m} r\right)+J_{1 / 2}\left(\mu_{l m} r\right), \\
\chi_{2, l m}(r) \approx J_{1 / 2}\left(\mu_{l m} r\right)-J_{-1 / 2}\left(\mu_{l m} r\right), \text { for } \nu=-1 / 2 .
\end{array}\right.
\end{gathered}
$$

Examples: chaotic Dirac AB billiard. The billiards $\mathcal{B}$ in the $w=u+i v$ plane can be transformed from a unit disk on the complex $z=x+i y$ plane through the conformal mapping

$$
w(z)=\frac{z+b_{1} z^{2}+b_{2} e^{i \delta} z^{3}}{\sqrt{1+2 b_{1}^{2}+3 b_{2}^{2}}},
$$

as shown in Fig. 10, where the parameters $b_{1}=0.49$ and $b_{2}=\delta=0$ correspond to the chaotic heart-shaped billiard, and $b_{1}=b_{2}=0.2$ and $\delta=\pi / 3$ are for the chaotic Africa billiard [254, 255]. The following gauge can be chosen to treat the magnetic flux, in which the lines of the vector potential $\boldsymbol{A}$ are the contours of a scalar function $F(u, v)$ :

$$
\boldsymbol{A}(u, v)=\frac{\Phi}{2 \pi}\left(\frac{\partial F}{\partial v}, \frac{-\partial F}{\partial u}\right)
$$


where $F$ satisfies [256]: $\nabla_{u v}^{2} F=-2 \pi \delta(u) \delta(v)$. The Hamiltonian for the confined Dirac particle is given in Eq. (11). Inside the billiard, the potential vanishes: $V(u, v)=0$. When being acted upon by the Hamilton operator $\hat{H}$ again, the Dirac equation [Eq. (71)] becomes

$$
\begin{array}{r}
\nabla_{u v}^{2} \psi(w)-2 i \alpha\left(\frac{\partial F}{\partial v} \frac{\partial}{\partial u}-\frac{\partial F}{\partial u} \frac{\partial}{\partial v}\right) \psi(w)+i \alpha \hat{\sigma}_{x} \hat{\sigma}_{y} \nabla_{u v}^{2} F- \\
\alpha^{2}\left[\left(\frac{\partial F}{\partial u}\right)^{2}+\left(\frac{\partial F}{\partial v}\right)^{2}\right] \psi(w)+k^{2} \psi(w)=0 .
\end{array}
$$

where $\alpha=\Phi / \Phi_{0}$ and $k=E /(\hbar c)$. Note that the term $i \alpha \hat{\sigma}_{x} \hat{\sigma}_{y} \nabla_{u v}^{2} F$ is particular to the Dirac AB billiard, which is not present in the Shrödinger AB billiard [256]. However, because of the relation $\nabla_{u v}^{2} F=-2 \pi \delta(u) \delta(v)$, it is singular at the origin and is zero otherwise. Practically, by setting a inner disk of radius $\xi \ll 1$ to having an infinite mass potential, the relevant billiard region excludes this singular point. The inclusion of the $A B$ flux can have two different types of boundary conditions about the singular point. Besides introducing an infinite mass boundary for the inner disk and letting the radius go to zero, which is relevant to the case where the AB flux only contributes to a global phase, there is a different setup for the boundary condition of the AB flux in the quantum field theory where further interactions need to be taken into account to calculate the vacuum energy $[252,253]$.

Transforming back to the disc region in the $z$-plane $\boldsymbol{r}=(x, y)$ based on $w(z)$ is straightforward. With $\psi(w)=\psi^{\prime}(z)=\psi^{\prime}(\boldsymbol{r})$, one obtains

$$
\begin{array}{r}
\nabla^{2} \psi^{\prime}(\boldsymbol{r})-2 i \alpha\left(\frac{\partial F}{\partial y} \frac{\partial}{\partial x}-\frac{\partial F}{\partial x} \frac{\partial}{\partial y}\right) \psi^{\prime}(\boldsymbol{r})- \\
\alpha^{2}\left[\left(\frac{\partial F}{\partial x}\right)^{2}+\left(\frac{\partial F}{\partial y}\right)^{2}\right] \psi^{\prime}(\boldsymbol{r})+k^{2}\left|w^{\prime}(z)\right|^{2} \psi^{\prime}(\boldsymbol{r})=0,
\end{array}
$$

where the last term includes the nonuniform part $\left|w^{\prime}(z)\right|^{2}$ originated from the chaotic boundary in the $w$ plane. In particular, $F$ can be chosen as $F(\boldsymbol{r})=-\ln |\boldsymbol{r}|$ in the $z$ plane, so in the polar coordinates, the above equation becomes

$$
\nabla^{2} \psi^{\prime}(r, \phi)-\frac{2 i \alpha}{r^{2}} \frac{\partial \psi^{\prime}(r, \phi)}{\partial \phi}-\frac{\alpha^{2}}{r^{2}} \psi^{\prime}(r, \phi)+k^{2}\left|w^{\prime}(z)\right|^{2} \psi^{\prime}(r, \phi)=0 .
$$

To solve this equation, one can expand $\psi^{\prime}$ in terms of the eigenfunctions $\psi_{l m}(r, \phi)$ of the circular Dirac AB billiard of the unit disc with a vanishing inner radius, whose corresponding eigenvalues are $\mu_{l m}$, with $l$ and $m$ being the relevant quantum numbers. One has

$$
\psi^{\prime}(r, \phi)=\sum_{l=-\infty}^{\infty} \sum_{m=1}^{\infty} c_{l m} \psi_{l m}(r, \phi),
$$

where $c_{l m}$ are the expansion coefficients. Substituting Eq. (102) into Eq. (101) leads to

$$
\frac{\nu_{l m}}{k^{2}}-\sum_{l^{\prime} m^{\prime}} M_{l m l^{\prime} m^{\prime}} \nu_{l^{\prime} m^{\prime}}=0,
$$

where $\nu_{l m}=\mu_{l m} c_{l m}$, and the elements of the matrix $\boldsymbol{M}$ are

$$
\begin{aligned}
M_{l m l^{\prime} m^{\prime}}= & \frac{N_{l m} N_{l^{\prime} m^{\prime}}}{\mu_{l m} \mu_{l^{\prime} m^{\prime}}} \int_{0}^{1} r \mathrm{~d} r \int_{0}^{2 \pi} \mathrm{d} \phi\left|w^{\prime}(z)\right|^{2} \cdot e^{i\left(l^{\prime}-l\right) \phi} \\
& \cdot\left\{\left[J_{\nu}\left(\mu_{l m} r\right)+\beta_{l m} Y_{\nu}\left(\mu_{l m} r\right)\right] \cdot\left[J_{\nu^{\prime}}\left(\mu_{l^{\prime} m^{\prime}} r\right)+\beta_{l^{\prime} m^{\prime}} Y_{\nu^{\prime}}\left(\mu_{l^{\prime} m^{\prime}} r\right)\right]\right. \\
+ & {\left.\left[J_{\nu+1}\left(\mu_{l m} r\right)+\beta_{l m} Y_{\nu+1}\left(\mu_{l m} r\right)\right] \cdot\left[J_{\nu^{\prime}+1}\left(\mu_{l^{\prime} m^{\prime}} r\right)+\beta_{l^{\prime} m^{\prime}} Y_{\nu^{\prime}+1}\left(\mu_{l^{\prime} m^{\prime}} r\right)\right]\right\} . }
\end{aligned}
$$


For the conformal transformation (99), the angular integration in Eq. (104) can be calculated analytically, which yields

$$
I=\int_{0}^{2 \pi} \mathrm{d} \phi\left|w^{\prime}(z)\right|^{2} \cdot e^{i\left(l^{\prime}-l\right) \phi}= \begin{cases}2 \pi\left(1+4 b_{1}^{2} r^{2}+9 b_{2}^{2} r^{4}\right) & l=l^{\prime} \\ 2 \pi\left(2 b_{1} r+6 b_{1} b_{2} r^{3} e^{ \pm i \delta}\right) & l=l^{\prime} \pm 1 \\ 2 \pi\left(3 b_{2} r^{2} e^{ \pm i \delta}\right) & l=l^{\prime} \pm 2\end{cases}
$$

Substituting $I$ into (104) and integrating over variable $r$, one obtains the $M$ matrix. Equation (103) can be written in the form of eigenequation:

$$
\boldsymbol{M} \cdot \boldsymbol{\nu}^{(n)}=\lambda_{n} \boldsymbol{\nu}^{(n)}
$$

Each eigensolution of the eigenequation yields a set of eigensolution of the original Dirac billiard. Specifically, from the eigenvector $\boldsymbol{\nu}^{(n)}$, one gets the expansion coefficients $c_{l m}^{(n)}=\nu_{l m}^{(n)} / \mu_{l m}$ with $l, m$ being the indices of the components. As a result, the eigen-wavefunction $\psi_{n}^{\prime}(r, \phi)=$ $\Sigma_{l m} c_{l m}^{(n)} \psi_{l m}(r, \phi)$ can be obtained, and $\psi_{n}(u, v)=\psi_{n}^{\prime}(x(u, v), y(u, v))$. From the eigenvalue $\lambda_{n}$, one gets the eigenwavevector $k_{n}=1 / \sqrt{\lambda_{n}}$, and consequently the eigenenergy $E_{n}=\hbar c k_{n}$. 


\section{ENERGY LEVEL-SPACING STATISTICS IN RELATIVISTIC QUANTUM CHAOTIC SYS- TEMS}

\section{A. Energy level-spacing statistics - an introduction}

The statistics of energy levels in quantum chaotic systems have been a "traditional" topic of study in nonrelativistic quantum chaos $[2,3,152]$. A fundamental result is that, for classically chaotic systems without any geometrical symmetry so that, in the corresponding quantum systems, there are neither degeneracies in the energy levels nor corrections among them, the energy level-statistics can be described by those of random matrices [4, 9, 153]. In particular, for systems that possess a time-reversal symmetry, the Hamiltonian matrix is real symmetric, a property that is preserved under orthogonal transformations. For such systems, the energy level-spacing statistics follow the distribution of those of random matrices from the Gaussian orthogonal ensemble (GOE) [2]. If, in addition to the time-reversal symmetry, the system has a half-integer spin interaction, then the Hamiltonian can be represented by quaternion real matrices that are invariant under symplectic transformations. In this case, the resulting level-spacing statistics follow those of random matrices from the Gaussian symplectic ensemble (GSE). When the time-reversal symmetry is broken, e.g., by a magnetic field, the Hamiltonian is invariant under the unitary transformations and, consequently, the level-spacing statistics are governed by the Gaussian unitary ensemble (GUE) random matrices. GOE statistics have been observed in many systems [2, 3, 152]. GUE statistics have also been observed experimentally in the microwave analog of quantum billiards that exhibit chaotic dynamics in the classical limit [257, 258], subject to an external magnetic field that breaks the time reversal symmetry. In fact, GUE statistics can arise for a subset of the eigenstates belonging to a particular symmetry class that breaks the time reversal symmetry [259, 260]. Recently, GUE statistics have been observed in yellow Rydberg excitons in cuprous oxide [261]. As a simulator of quantum graphs, microwave networks have been used to probe into the symmetry properties of quantum complex systems, where both GUE [262, 263] and GSE [264] statistics have been observed experimentally.

In the vast existing literature on quantum chaos, level spacing statistics were mostly studied in the realm of nonrelativistic quantum systems. A natural question is whether the distinct statistical behaviors associated with different symmetry classes are preserved in relativistic quantum chaotic systems. The problem is highly nontrivial as there are basic traits in relativistic quantum systems such as spin, linear dispersion, Klein tunneling, and chirality, etc., which are not encompassed by the Schrödinger equation in nonrelativistic quantum systems. An early concern was whether this problem has any physical correspondence because, from an experimental point of view, a relativistic particle cannot be confined within a designed region due to the Klein tunneling effect $[265,266]$. Especially, while in a regular system the tunneling probability may be low due to the fact that it occurs only for a specific set of incident angles on the domain boundary, in a chaotic system a broad range of the angles can arise. The tremendous development of twodimensional Dirac materials in the past decade has removed the concern, as it is indeed possible to confine quasiparticles of Dirac characteristics in experimental systems of graphene or topological insulators. Especially, graphene confinements that have the geometric shape of chaotic billiards represent a potential experimental system for testing energy-level statistics in the relativistic quantum regime.

A seminal work in this area is that of Berry and Mondragon [93] on the massless neutrino 
billiard. (Note that a recent experiment revealed that neutrinos have a minuscule but nonzero mass [267].) Specifically, for a massless spin-half particle in a two-dimensional closed domain with infinite mass confinement, e.g., the Dirac billiard, the level-spacing statistics are of the Poisson type if the classical dynamics are integrable, a result that is similar to that in nonrelativistic quantum systems. However, for classically chaotic dynamics, the level-spacing statistics are persistently those of GUE, even in the absence of any magnetic field, representing a kind of anomaly as, in nonrelativistic quantum systems, the statistics under such a setting would be of the GOE type. The occurrence of GUE statistics in the absence of any magnetic field can be attributed to the chiral nature of Dirac particles and the confinement scalar potential that breaks the time-reversal symmetry. At the time of writing, such phenomena have not been tested experimentally, partly due to the difficulty in constructing relativistic quantum systems with chaotic classical dynamics in the laboratory. Note, however, for three-dimensional chaotic Dirac billiards, the level-spacing statistics are still of the GOE type [268].

Here we review the relevant works on level-spacing statistics for both graphene billiards with confined pseudo-Dirac particles and two-dimensional massless Dirac billiards. There are some differences between the two types of billiard systems. For graphene, the pseudo-particles about a single Dirac point follow the same massless Dirac equations, and the boundary conditions are different. While in an open graphene system electrons associated with the two valleys can be separated [269-271], for a closed graphene billiard scattering at the boundary effectively "couples" the two valleys, generating phenomena that are more likely to correspond to those in systems of two coupled Dirac fermions. Graphene systems can thus exhibit phenomena that are not shared by either nonrelativistic quantum or purely relativistic quantum systems, and the energy level statistics may have implications to transport in graphene-based devices such as quantum dots [272-275], a kind of "open" billiard structure.

\section{B. Characterization of level-spacing statistics}

The primary quantity to characterize energy level-spacing statistics is the probability distribution function of the nearest neighbor spacings. Suppose the energy spectrum $\left\{E_{n}\right\}$ for a closed quantum system has been calculated and ordered as $E_{1}<\ldots<E_{n}<E_{n+1}<\ldots$.. For different systems or even a different energy range of the same system, the density of states can be different. It is thus convenient to unfold the energy spectrum so that the fluctuation statistics do not depend on the system details. This can be done by introducing the integrated density of states $N\left(E_{n}\right)$, e.g., the number of energy levels below $E_{n}$, so the unfolded spectrum can be defined as $x_{n} \equiv\left\langle N\left(E_{n}\right)\right\rangle$, where $\langle N(E)\rangle$ is the smoothed version of $N(E)$. With the value of $x_{n}$ so defined, the energy spacing is given by $S_{n}=x_{n+1}-x_{n}$. Let $P(S)$ be the distribution function of $S_{n}$ with the normalization conditions $\int P(S) \mathrm{d} S=1$, then the average spacing for the unfolded spectrum is automatically one, i.e. $\int S P(S) \mathrm{d} S=1$. For nonrelativistic quantum billiards, depending on the nature of the corresponding classical dynamics and the system symmetry, the distribution of the unfolded level-spacings follows a number of universal classes. In particular, for classically integrable systems, the distribution is Poisson [276]:

$$
P(S)=e^{-S} \text {. }
$$

If the system exhibits fully developed chaos in the classical limit and it does not possess any geometric symmetry but the time reversal symmetry is preserved, the level-spacing distribution 
follows the GOE statistics [277]:

$$
P(S)=\frac{\pi}{2} S e^{-\pi S^{2} / 4}
$$

When the time reversal symmetry is broken (e.g., in the presence of a magnetic field), GUE statistics arise:

$$
P(S)=\frac{32}{\pi} S^{2} e^{-(4 / \pi) S^{2}}
$$

The cumulative level-spacing distribution is given by

$$
I(S)=\int_{0}^{S} P\left(S^{\prime}\right) \mathrm{d} S^{\prime}
$$

Another often used characterizing quantity is the spectral rigidity $\Delta_{3}(L)$ that quantifies the longrange spectral fluctuations, which is defined as [278]

$$
\Delta_{3}(L)=\left\langle\min (a, b) L^{-1} \int_{-L / 2}^{L / 2} \mathrm{~d} x\left\{N\left(x_{0}+x\right)-a x-b\right\}^{2}\right\rangle,
$$

where the average is over $x_{0}$. Numerically, if $n$ unfolded levels $\widetilde{x}_{i}=x_{i}-x_{0}$ lie in the interval $[-L / 2, L / 2]$, e.g., $-L / 2 \leq \widetilde{x}_{1} \leq \cdots \leq \widetilde{x}_{n} \leq L / 2$, the integral in Eq. (110) can be carried out to yield [279]

$$
\begin{aligned}
\Delta_{3}\left(L, x_{0}\right) & =\frac{n^{2}}{16}-\frac{1}{L^{2}}\left[\sum_{i=1}^{n} \widetilde{x}_{i}\right]^{2}+\frac{3 n}{2 L^{2}}\left[\sum_{i=1}^{n} \widetilde{x}_{i}^{2}\right]-\frac{3}{L^{4}}\left[\sum_{i=1}^{n} \widetilde{x}_{i}^{2}\right]^{2} \\
& +\frac{1}{L}\left[\sum_{i=1}^{n}(n-2 i+1) \widetilde{x}_{i}\right]
\end{aligned}
$$

and $\Delta_{3}(L)=\left\langle\Delta_{3}\left(L, x_{0}\right)\right\rangle_{x_{0}}$. For a correlated unfolded energy level sequence, theoretically the quantity $\Delta_{3}(L)$ is given by

$$
\Delta_{3}(L)=\frac{1}{15 L^{4}}\left[L^{5}-\int_{0}^{L} \mathrm{~d} u(L-u)^{3}\left(2 L^{2}-9 L u-3 u^{2}\right) Y(u)\right]
$$

where $Y(u)$ is the two-level cluster function defined as [280]:

$$
\begin{aligned}
& Y(u)=0 \quad \text { for Poisson } \\
& Y(u)=U(u)^{2}+\frac{\mathrm{d}}{\mathrm{d} u} U(u) \times \int_{u}^{\infty} \mathrm{d} t U(t) \text { for GOE, } \\
& Y(u)=U(u)^{2} \quad \text { for GUE, }
\end{aligned}
$$

and $U(u) \equiv \sin (\pi u) /(\pi u)$.

Phenomenologically, one can relate the Poisson and GOE distributions through the function [281]

$$
P_{H}(S ; \alpha, \beta)=N \frac{\rho S e^{-\rho S-(\alpha \rho S)^{2} / 2}}{\sqrt{\rho^{2} S^{2} e^{-(\alpha \rho S)^{2}}+\beta^{2} e^{-2 \rho S}}},
$$

where $\rho$ and $N$ are determined by the normalization conditions $\int P_{H} d S=\int S P_{H} d S=1, \alpha$ is a system-specific constant, and $\beta$ is a parameter that can be tuned to generate distinct statistics. In particular, one gets the Poisson and GOE statistics for $\beta=0$ and $\beta \rightarrow \infty$, respectively. 

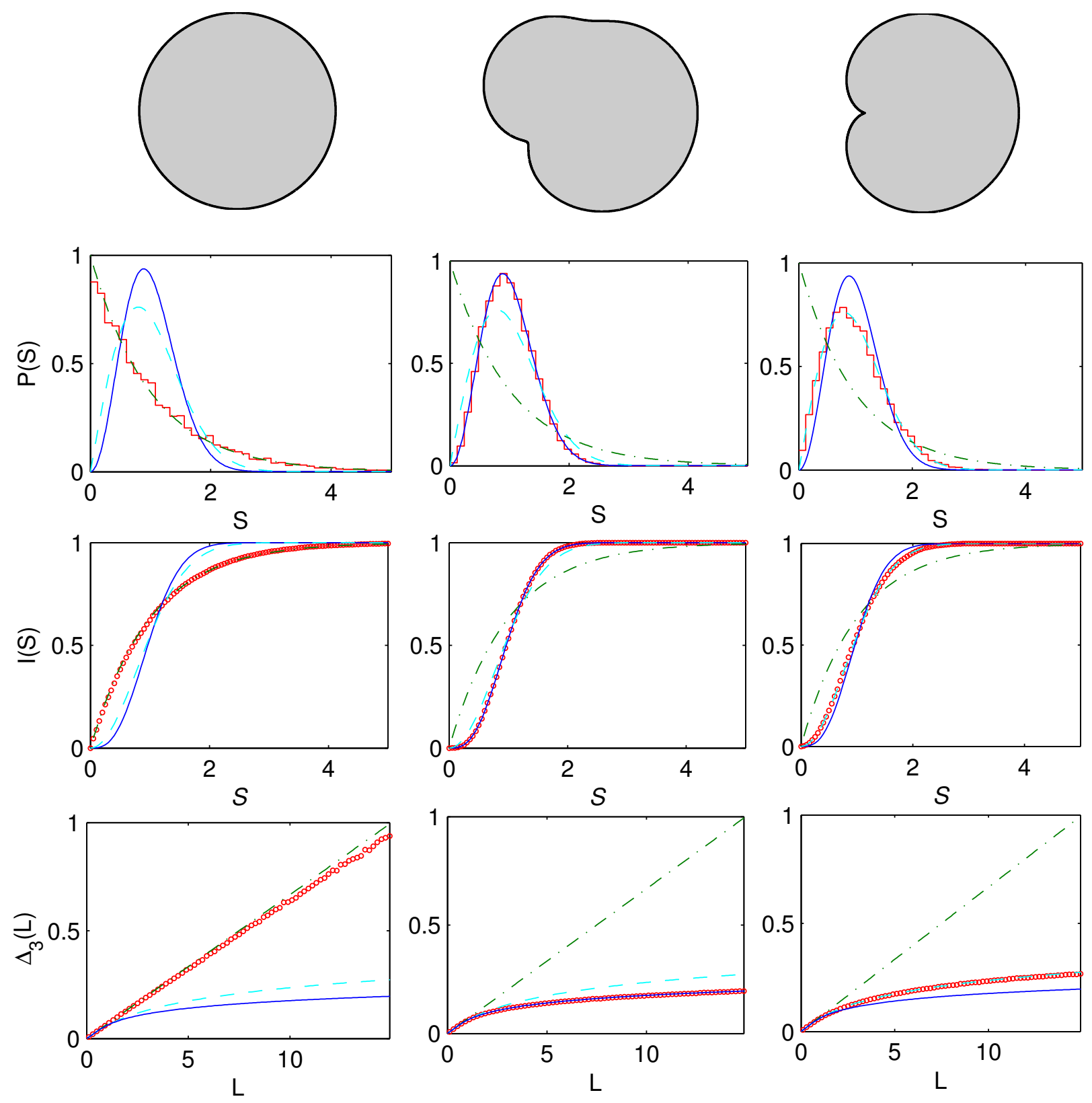

FIG. 11. Level spacing statistics of Dirac billiards. Left column: an integrable circular domain of unit radius; middle column: the chaotic Africa billiard; right column: a chaotic heart-shaped billiard. The first row shows the shape of the billiards. The second to the fourth rows are the unfolded level-spacing distribution $P(S)$, the cumulative level-spacing distribution $I(S)$, and the spectral rigidity $\Delta_{3}(L)$, respectively. The green dash-dotted lines, cyan dashed lines, and blue solid lines indicate the corresponding results associated with the Poisson, GOE, and GUE statistics, respectively. For each billiard, the red symbols are numerical results from 13000 energy levels. From Ref. [282] with permission. 


\section{Dirac billiards - GUE statistics without a magnetic field}

In nonrelativistic quantum systems, classical chaos typically leads to GOE statistics in energy level-spacings. However, for a massless particle in a relativistic quantum system, the level-spacing statistics are of the GUE type even without any magnetic field [93]. In particular, Berry and Mondragon analyzed the confined neutrino billiard described by the massless Dirac equation in a closed domain (Sec. IIC 1) and calculated the eigenenergies by using the boundary integral method. To contrast with classical integrable and chaotic dynamics, they considered a circular domain of unit radius (left columns of Fig. 11) and the Africa billiard [5] (middle columns of Fig. 11) with boundary described by the following conformal mapping of the unit circle:

$$
w(z)=\left(z+0.2 z^{2}+0.2 z^{3} e^{i \pi / 3}\right)
$$

where $z$ is the complex variable describing the unit circle $|z|=1$. Classically, the Africa billiard gives rise to fully developed chaotic motion, and it has no mirror or other discrete symmetries. To get a more complete picture, here we also consider a heart-shaped billiard (right columns of Fig. 11) whose boundary is given by

$$
w(z)=\frac{1}{\sqrt{1+2 \beta^{2}}}\left(z+\beta z^{2}\right), \beta \in[0,1 / 2) .
$$

For $\beta=0.49$, a previous work [249] demonstrated chaos. Note that the heart-shaped billiard has an up-down mirror symmetry.

For a nonrelativistic quantum billiard, the Weyl formula gives the smoothed wave-vector staircase function $[2,3]$ as

$$
\langle\mathcal{N}(k)\rangle=\frac{\mathcal{A} k^{2}}{4 \pi}+\gamma \frac{\mathcal{L} k}{4 \pi}+\cdots,
$$

where $\mathcal{A}$ and $\mathcal{L}$ are the area and perimeter of the billiard, respectively, $\gamma=-1$ (or 1 ) is for the Dirichlet (or Neumann) boundary condition. For the relativistic Dirac billiard, Berry and Mondragon showed [93] that $\gamma=0$, so there is no perimeter correction to the staircase function.

For the three types of relativistic quantum billiards in Fig. 11, the eigenenergies and eigenfunctions can be calculated efficiently and accurately by the conformal mapping method [123]. For each of the three types of billiards, representative results from a statistical analysis of more than 13000 energy levels are shown in Fig. 11. From the various statistical indicators, one can see that the level-spacing statistics are Poisson for the integrable billiard. For the chaotic Africa billiard that has neither any geometric symmetry nor time reversal symmetry, GUE statistics arise. For the chaotic heart-shaped billiard, because it has a mirror symmetry and is invariant under the combination of time-reversal and reflection operations, the level-spacing statistics are of the GOE type.

While the conformal mapping method [123] is computationally efficient and accurate, a limitation is that it is applicable only to those domains that can be mapped from a circular domain. For a relativistic quantum billiard of arbitrary shape, the boundary integral method can be employed (Sec. II E). In addition, Ni et al. developed a direct discretization method (Sec. IID) to solve the spectrum and eigenstates of a massless Dirac particle with infinite mass confinement in the presence of an electric potential, e.g., a potential barrier inside the domain [122, 134, 135]. Results similar to those in Fig. 11 were obtained. 


\section{Graphene billiards}

\section{Basics}

Qualitative [237] and also some quantitative insights [199] into the band structure of an infinitely extended graphene ribbon can be gained through the conventional tight-binding model, based on the assumption that the interactions of the $p_{z}$ orbitals are non-negligible only for nearest, second-nearest and third-nearest neighbors. The same model can be used to determine the energy levels in a finite-size graphene sheet. The dimension of the associated tight-binding Hamiltonian coincides with the number of atoms forming the graphene sheet, with each diagonal element corresponding to one atom. This entails imposing the Dirichlet boundary condition along the first row of atoms outside the boundary of the graphene billiard. Taking into account couplings between neighboring atoms within a certain range, a tight-binding Hamiltonian can be obtained [101-107]. Diagonalizing the Hamiltonian matrix yields the energy eigenvalues and eigenfunctions.

The tight-binding Hamiltonian for a graphene billiard is given by

$$
\hat{H}=\sum\left(-\varepsilon_{i}\right)|i\rangle\left\langle i\left|+\sum\left(-t_{i j}\right)\right| i\right\rangle\langle j|,
$$

where the first summation is over all the atoms and the second summation is over all pairs of neighboring atoms including the nearest and possibly the second- or third-nearest neighboring pairs, with the respective hopping energies $t_{i j}$ determined by the distance and by the consideration of whether boundary atoms are involved. In the absence of any impurity, the on-site energy $\varepsilon_{i}$ is identical for all atoms and it contributes a uniform shift to the energy spectrum, which can be removed from consideration. In the presence of random disorders, a proper distortion potential can be added to the on-site energy. A magnetic field induces a phase factor associated with the hopping energy:

$$
\widetilde{t}_{i j}=t_{i j} \exp \left(-i \frac{2 \pi}{\Phi_{0}} \int_{\boldsymbol{r}_{j}}^{\boldsymbol{r}_{i}} \mathrm{~d} \boldsymbol{r} \cdot \boldsymbol{A}\right),
$$

with $\boldsymbol{A}$ being the vector potential.

To reveal the relativistic quantum nature of the quasiparticle motions in graphene, in the studies of level-spacing statistics, one usually focuses on the energy levels about the Dirac points. Figure 12 shows the energy-wavevector relation for an infinite graphene flake without any magnetic field. As can be seen from the contour lines around each Dirac point, when the energy is low, e.g., $E / t=0.2$, the contour line is almost circular, indicating that the $E-\boldsymbol{k}$ relation is isotropic, which is characteristic of relativistic quantum behaviors. For larger energy values, e.g., $E / t=0.6$, trigonal warping distortions arise [271, 283-285], leading to an anisotropic $E-\boldsymbol{k}$ relation bearing the hexagonal symmetry of the graphene lattice. The distortions become more dominant as the energy is increased further.

\section{Effects of disorders and edge roughness}

The effects of random disorders on the level-spacing statistics were studied [119, 273, 275, 286-288]. For example, the level-spacing statistics of a rectangular phase-coherent graphene flake were calculated [286] in the presence of random disorders such as edge roughness $\Delta W$, short-range disorder $n_{D}$ due to randomly distributed point defects in the interior, and long-range 


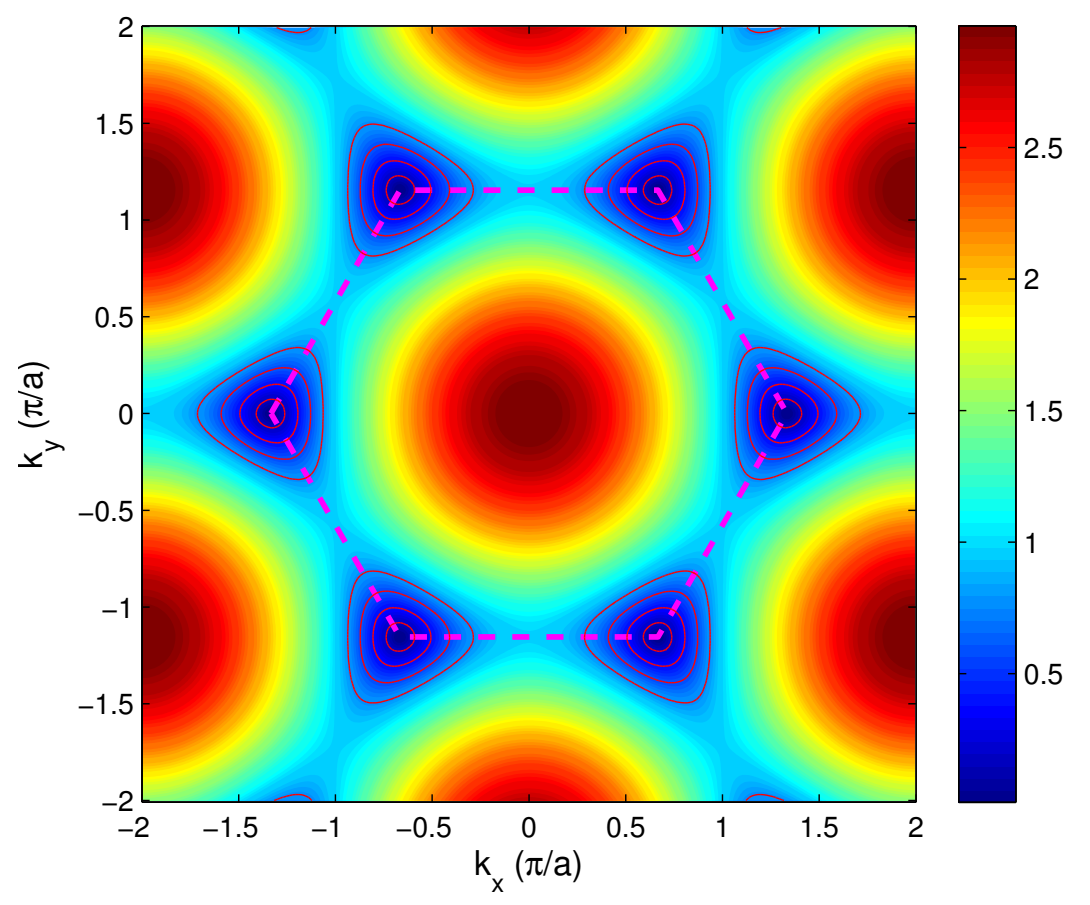

FIG. 12. Contour plot of energy in the momentum space for an infinite graphene flake. The zigzag direction is along $k_{x}$. The dashed line indicates the first Brillouin zone. The four sets of solid contour lines around each individual Dirac point are for $E / t=0.2,0.4,0.6,0.8$ (inside out), respectively. The lattice constant is $a=\sqrt{3} a_{0}=2.464 \AA$. From Ref. [118] with permission.

screened Coulomb distortion $n_{C}$ due to charge deposition on either the substrate or the flake. The system has a linear dimension of 10-40 $\mathrm{nm}$. To reproduce the graphene band structure, a third nearest-neighbor tight-binding approximation is necessary [199]. The modified C-C bond length at the flake boundary is accounted for by increasing the nearest-neighbor coupling to the out-most carbon atoms by $12 \%$ in accordance with the ab initio density-functional calculation [289]. The spectrum of the graphene flake was determined using the Lanczos algorithm [290], giving rise to 500 eigenstates closest to the Dirac point. Ensemble averages using 5000 disorder realizations were used. After unfolding the spectrum, the authors found [286], for the ideal rectangular graphene dot, a near-perfect Poisson distribution for the level spacings. By gradually increasing either the edge roughness or the defect concentration, the distribution smoothly evolves into a GOE statistic. For moderate values of the edge roughness amplitude $W=0.4 \mathrm{~nm}$, a Schrödinger and a graphene system of the same geometry display a markedly different level-spacing statistics: while for the Schrödinger system the level-spacing statistics have already reached the GOE limit, for the graphene counterpart the statistics are still close to the Poisson limit, indicating the unique spectral properties of graphene. Representative results are shown in Fig. 13.

The statistical results can also be fitted to the two-parameter Hasegawa distribution [281] [Eq. (113)], where a larger value of the fitting parameter $\beta$ indicates a better fit to the GOE distribution. The value of the parameter $\alpha$ in Eq. (113) was chosen to be 0.75 to correctly reproduce the numerically obtained level-spacing distributions for different values of edge roughness. Relatively strong edge roughness of $2 \mathrm{~nm}$ (or short-range impurity concentration $n_{D}=5 \times 10^{-3}$ and longrange concentration $n_{C}=2 \times 10^{-2}$ ) is required for the GOE statistics, where $n_{D}$ and $n_{C}$ are given 

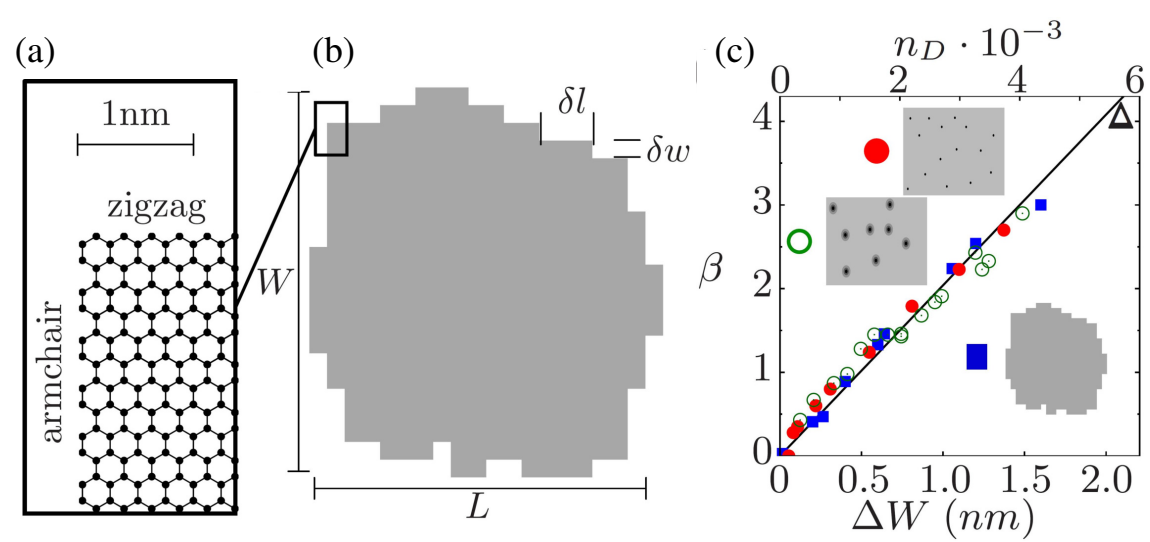

FIG. 13. Level spacing statistics of a graphene flake in the absence or presence of random disorders. (a) A rectangular segment of a graphene flake with armchair vertical and zigzag horizontal edges. (b) Approximately rectangular quantum dot with rough edges. (c) Dependence of parameter $\beta$ characterizing the transition from a Poisson to a GOE distribution on the edge roughness amplitude $\Delta W$ (squares), and the density of short-range ( $n_{D}$, full circles) or of long-range $\left(n_{C}=4 n_{D}\right.$, rescaled for visual clarity only, open circles) impurities. From Ref. [286] with permission.

in units of impurities per carbon atom, whose values are typically within [291] $1.8 \times 10^{-3}$. Through fitting with the Hasegawa distribution, the authors uncovered [286] a linear relation between $\beta$ and a parameter characterizing the disorder distribution: $\beta \approx 2 \Delta W$, where $\Delta W$ measures the edge roughness, or $\beta \approx 0.7 n_{D}$, or $\beta \approx 0.2 n_{C}$, as shown in Fig. 13(c). Such a linear relation can be used to estimate the strength of the disorder in the graphene flake. For example, using the data in [273], the authors found [286] $\beta \approx 4$, indicating the presence of disorders of strength $\Delta W \approx 2 \mathrm{~nm}$ or $n_{D} \approx 5.5 \times 10^{-3}$.

There were also works on disorders in graphene nanotubes. For example, in Ref. [287], a theoretical model based on the nearest neighbor hopping energy was studied, where uniformly distributed random disorders were introduced into either the on-site energies [the first term of Eq. (115)] or the nearest neighbor hopping energies [the second term of Eq. (115)]. For zero disorder strength, the level-spacing statistics were found to be of the Poisson type, as expected. When disorders even of relatively weak strength are present, level repulsion arises and the statistics become the GOE type. For the extreme case of wide and short finite nanotube dots, i.e., $W / L \gg 1$ (the opposite limit to conventional nanotubes), pseudo-diffusive behavior arises even for infinitesimally small disorder strength. For narrow, long cylindrical nanotubes $(W / L \ll 1)$, the corresponding level statistics are of the ballistic $\delta$-function type, which are similar to those arising from the system of a one-dimensional chain of interacting particles. For strong disorder, i.e., when the energy fluctuations are comparable to the hopping energy, Poisson statistics reappear due to the onset of Anderson localization [287]. In a recent study [288] of weakly disordered graphene flakes with zigzag edges, it was found that the level-spacing distribution for the first two positive energy bands in the Dirac region is neither GOE nor Poisson, but is similar to that at the critical point of the metal-insulator transition.

In general, quantum transport in a finite graphene nanoribbon can occur through the edge states as in a topological insulator. For example, conductance measurement [275] of a graphene nanoribbon quantum dot of size $500 \mathrm{~nm} \times 60 \mathrm{~nm}$ at temperature $20 \mathrm{mK}$, taking into account resonance 
peaks at zero bias, suggests level repulsion with GOE statistics. A plausible explanation is the presence of ripple type of disorders that introduce random scatterings and mix quantum states associated with different levels.

\section{Effects of chaos on level-spacing statistics in graphene systems}

There was an early work [273] on the role of chaos in level spacing statistics of graphene quantum dots. For large size (e.g., $>100 \mathrm{~nm}$ ), the dot behaves as a conventional single-electron transistor, exhibiting periodic Coulomb blockade peaks. For small size (e.g., $<100 \mathrm{~nm}$ ), the peaks become aperiodic, indicating the dominant effect of quantum confinement. It was found that random peak spacings and their statistics are well described by the theory of chaotic quantum billiards. Similar features were also observed in a tunable graphene single electron transistor [274].

A systematic study of the effect of chaos on the level-spacing statistics in graphene billiards was subsequently carried out $[115,118]$ using two billiard shapes that had commonly been used in previous studies of level-spacing statistics, the Africa billiard [5] and one eighth of the Sinai billiard, where the nearest neighbor interacting, tight-binding Hamiltonian was used. The boundary of the Africa billiard is described by $x+i y=64\left(z+0.2 z^{2}+0.2 z^{3} e^{i \pi / 3}\right) a$ with $a=\sqrt{3} a_{0}=2.46 \AA$ and $z$ denoting the unit circle in the complex plane. To attain the short wavelength regime, a relatively large billiard size is necessary, e.g., with area larger than $900 \mathrm{~nm}^{2}$ containing over 35000 atoms.
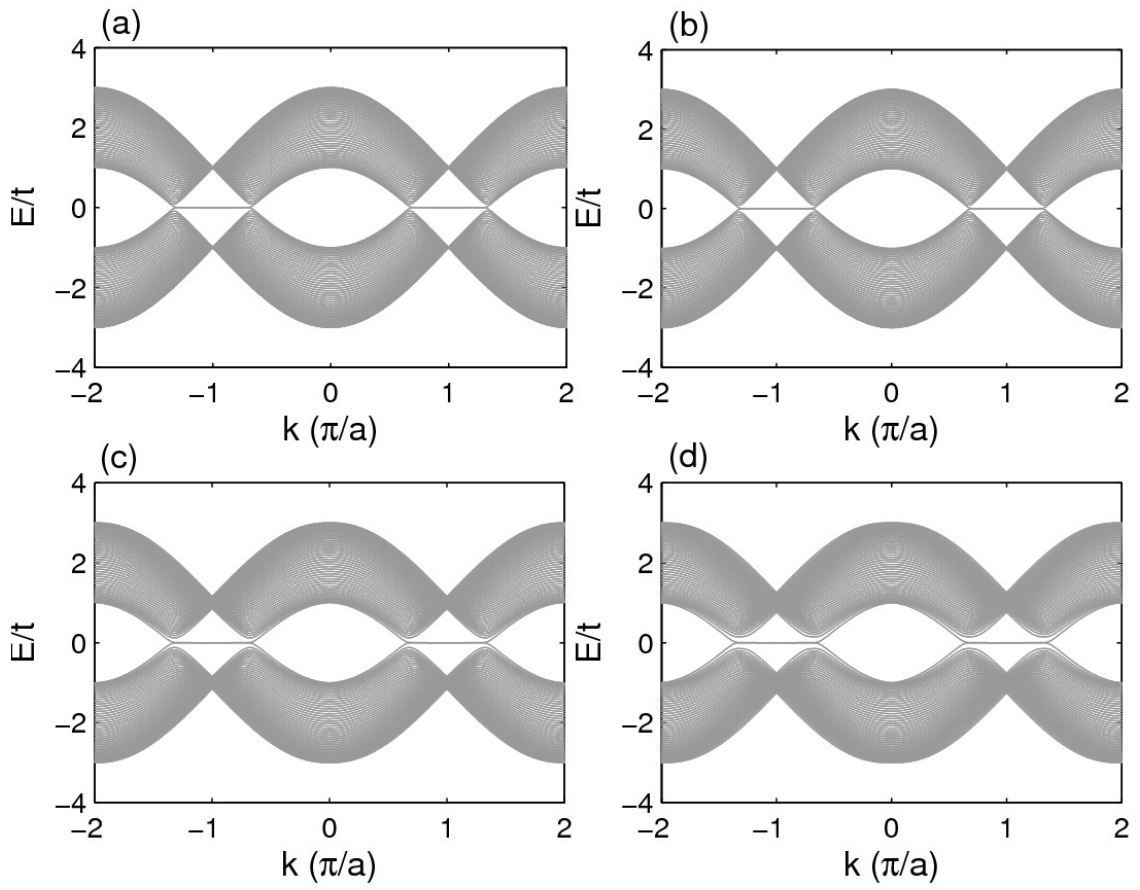

FIG. 14. Energy-momentum relation of a graphene nanoribbon with zigzag horizontal boundaries and the effect of a magnetic field. The magnetic field strength is measured by the magnetic flux $\Phi$. The dispersion relations in (a-d) are for $\Phi=0, \Phi=1 / 8000 \Phi_{0}, \Phi=1 / 800 \Phi_{0}$, and $\Phi=1 / 500 \Phi_{0}$, respectively. Each layer of the ribbon contains 100 atoms. From Ref. [118] with permission.

The effect of a magnetic field was also considered, where three cases were studied $[115,118]$ : magnetic flux $\Phi=0$ (absence of magnetic field), $\Phi=1 / 8000 \Phi_{0}$ (weak magnetic field), and 
$\Phi=1 / 800 \Phi_{0}$ (strong magnetic field). The magnetic field strength can be scaled down for larger confinements to yield similar effects. Basic insights can be gained by examining the energy band structure of a graphene nanoribbon. For example, Fig. 14 shows the band structure of a zigzag graphene nanoribbon (a) without and (b-d) with a magnetic field. In the presence of a sufficiently strong magnetic field, the original linear $E-\boldsymbol{k}$ relation in the small energy regime no longer holds, as shown, e.g., in Fig. 14(d). To focus on the relativistic quantum manifestations, it is necessary to choose the parameters such that the linear $E-\boldsymbol{k}$ relation is preserved even in the presence of a magnetic field [115, 118].
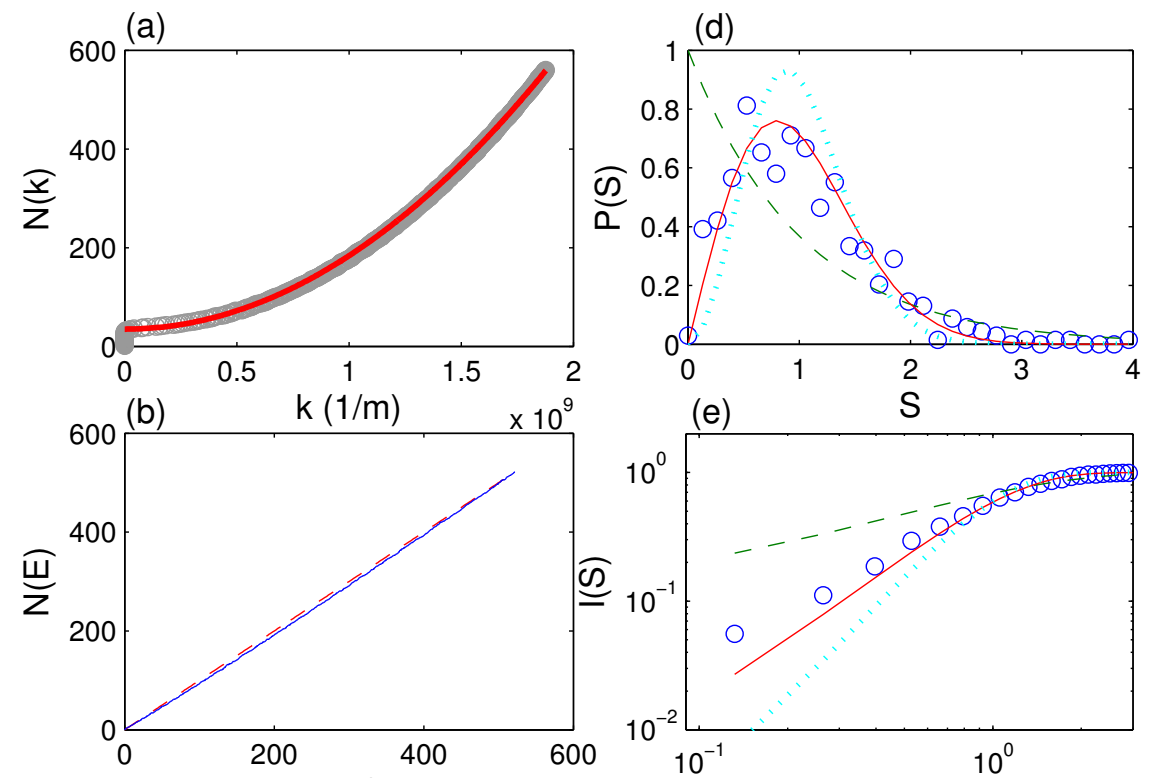

(e)
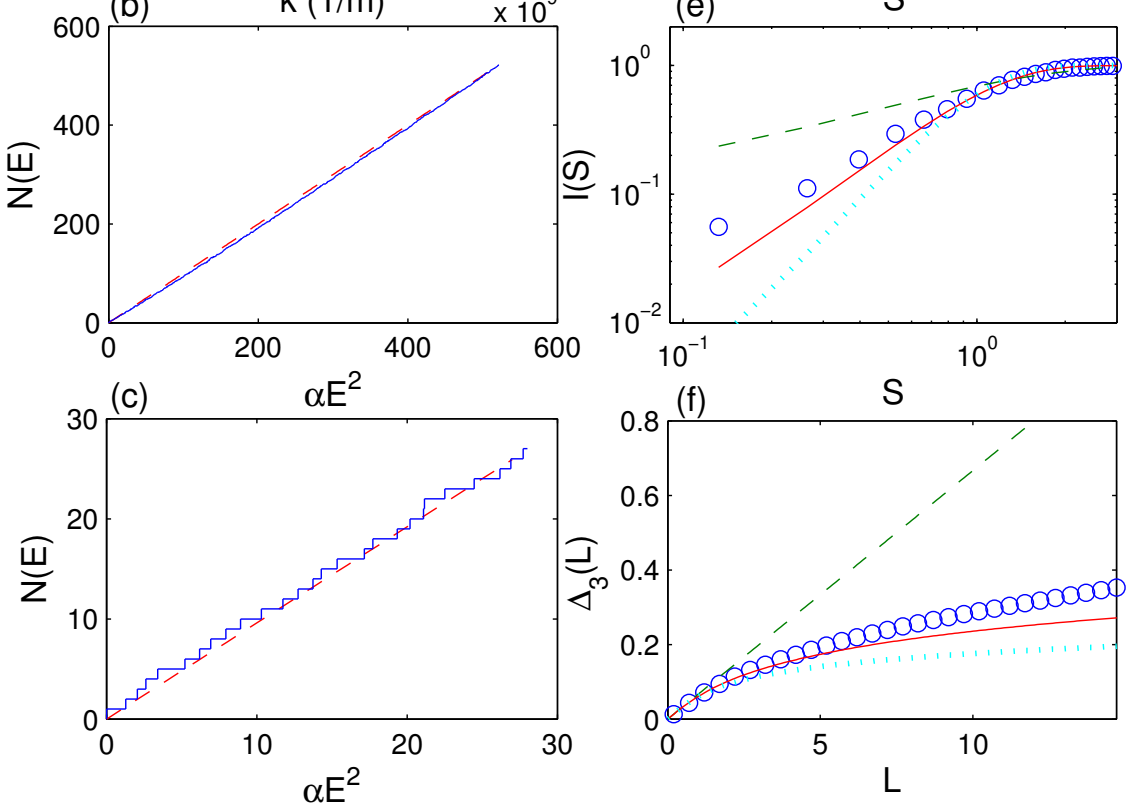

FIG. 15. Level-spacing statistics for the Africa shaped graphene billiard without any magnetic field $(\Phi=0)$. (a) Wavevector staircase function $N(k)$ for eigenenergies in the range $0<E_{n} / t<0.4$, where the number of energy levels is 560 (circles). The curve is given by $\langle N(k)\rangle=A k^{2} /(2 \pi)+35$ [Eq. (118)]. (b) Spectral staircase function $N(E)$ versus $\alpha E^{2}$ for $0.02<E_{n} / t<0.4$ with 522 levels, where $\alpha$ is the unfolding normalization constant. The dashed straight line is the averaged staircase function Eq. (119). (c) Magnification of part of (b) for $0.02<E_{n} / t<0.1$ with 28 levels. (d) Unfolded level-spacing distribution $P(S)$. (e) Cumulative unfolded level-spacing distribution $I(S)$. (f) Spectral rigidity $\Delta_{3}$. In (d-f), the numerical data are represented by open circles and the lines are theoretical predictions from the random matrix theory: dashed line for Poisson, solid line for GOE, and dotted line for GUE statistics. From Ref. [118] with permission. 
Level spacing statistics without a magnetic field. For a relativistic chaotic quantum billiard described by the Dirac equation, the smoothed wavevector staircase function for positive eigenvalues is given by [93] Eq. (114) with $\gamma=0$ :

$$
\langle N(k)\rangle=A k^{2} / 4 \pi+C_{1}+\ldots,
$$

where $C_{1}=-1 / 12$. For a chaotic graphene billiard, about a Dirac point, the energy-momentum relation is $E=\hbar v_{F} k=\sqrt{3} t a k / 2$ with $v_{F}=\sqrt{3} t a / 2 \hbar$ being the Fermi velocity. For the $n$th energy level $E_{n}$, one has

$$
k_{n}=\frac{2}{\sqrt{3} a} \cdot \frac{E_{n}}{t} .
$$

Once the eigenenergy $E_{n}$ is determined, the corresponding wave vector $k_{n}$ can be obtained. For the graphene Africa billiard, Fig. 15(a) shows the wavevector staircase function for eigenenergies in the range $0<E_{n} / t<0.4$, where the solid curve is given by

$$
\langle N(k)\rangle=A k^{2} / 2 \pi+C_{1}^{\prime}
$$

with $C_{1}^{\prime}=35$ being a fitting constant. Equation (118) differs from Eq. (117) in the leading "Weyl" term by a factor of 2 . The reason is that, for a single Dirac point, $\langle N(k)\rangle$ follows Eq. (117) but graphene has two non-equivalent Dirac points, so $\langle N(k)\rangle$ should be twice of that given by Eq. (117). The fitting constant $C_{1}^{\prime}$ is due to the edge states associated with the segments of the zigzag boundaries of the graphene billiard where their energies are all about zero. For a zigzag ribbon, the edge states exist for $E<E_{c}=\hbar v_{F} / L=\sqrt{3} t a /(2 L)$, where $L$ is the width of the ribbon [292]. The sizes of the graphene billiards are about $100 a$, leading to $E_{c} \simeq 0.01 t$. The edge states are localized on segments of zigzag boundaries. These states are degenerate, contributing to an artificial bias in the spectral staircase function for small energy values. For convenience, a minimum value of $2 E_{c}=0.02 t$ for $E_{n}$ can be set to remove the influence of the edge states.

Because of the linear energy-momentum relation $E=\hbar v_{F} k$, the smoothed spectral staircase function is given by

$$
\langle N(E)\rangle=\frac{A E^{2}}{2 \pi \hbar^{2} v_{F}^{2}}+C_{2}=\alpha E^{2}+C_{2},
$$

where $\alpha=A /\left(2 \pi \hbar^{2} v_{F}^{2}\right)$ is the unfolding normalization parameter and $C_{2}$ is now zero after setting $2 E_{c}$ for the minimum value of $E_{n}$. Figure 15(b) shows the spectral staircase function of $E_{n}$ for $0.02<E_{n} / t<0.4$, and Fig. 15(c) shows a magnification of part of Fig. 15(b) for eigenenergies in the range $0.02<E / t<0.1$. The dashed lines in these two panels are Eq. (119) with $C_{2}=0$. They agree well with the numerical results. The distribution of the unfolded level spacing for the Africa graphene billiard is shown in Fig. 15(d) and the cumulative distribution is presented in Fig. 15(e), which suggest GOE type of statistics. Figure 15(f) shows the spectral rigidity calculated from the available eigenenergies. This, together with Figs. 15(d,e), is strong evidence that the levelspacing distribution in a chaotic graphene billiard in the relativistic quantum regime follows the GOE statistics. Similar plots were obtained [115, 118] for one-eighth of the chaotic Sinai billiard with 37401 atoms and an area of $A=1.607 \times 10^{4} a^{2}=972 \mathrm{~nm}^{2}$, which also exhibit characteristics of GOE statistics.

The GOE statistics of unfolded level-spacings for graphene billiards can be understood, as follows. For the Dirac (neutrino) billiard, the time reversal symmetry is broken due to chirality. Due to the two non-equivalent Dirac points (valleys) in graphene, the time reversal symmetry for 
the Dirac system corresponds to the symplectic symmetry for graphene, which is essentially the time reversal symmetry for a single valley [266]. Thus the breaking of the time reversal symmetry in the neutrino billiard dose not imply time-reversal symmetry breaking in the graphene billiard. More specifically, in graphene, quasiparticles in the vicinity of a Dirac point obey the same Dirac equation as that for neutrino, but the confinement to realize the billiard plays a different role, where the abrupt edge termination couples the two valleys in the momentum space. As a result, the wavefunctions for quasiparticles with wavevectors near the two Dirac points are not separable, rendering invalid the description of the two-component spinor Dirac equation for the whole system. A full set of equations taking into account the two nonequivalent Dirac points and the boundary conditions are thus necessary to describe the motions of the relativistic quasiparticle in graphene. Typically, the time reversal symmetry is preserved [266], suggesting that the level-spacing statistics belong to either the GOE or the GSE class. The abrupt edge termination in a graphene billiard can be described by a step function in the form of an infinite potential at the edge. Since the range of the potential is short, the two valleys in the momentum space are coupled, which also breaks the sublattice symmetry. Since both the pseudospin valley and the sublattice symmetries are broken, Kramers degeneracy and GSE statistics can be ruled out [293]. The resulting level-spacing statistics are then of the GOE type. Similar effects were noticed by Robnik and Berry [6] that, although the system under consideration may possess neither time-reversal nor geometric symmetry (or other dynamical symmetries), it can be invariant under the combination of the two symmetries, and non-trivial representations can be found in which the Hamiltonian matrix elements are real, leading to GOE statistics (other than GUE as in the chaotic neutrino billiard [93]).

In realistic situations a number of nonideal factors can arise. The issue of robustness of the level-spacing statistics of chaotic graphene billiards is thus critical to experimental observation in the presence of perturbations such as the interactions beyond the nearest neighbors, lattice deformation, boundary bonds and staggered potentials caused by substrates. A numerical study $[115,118]$ revealed that the GOE statistics are quite robust against these nonideal factors.

Level spacing statistics in the presence of a magnetic field. A magnetic field breaks the time reversal symmetry of graphene [266] and causes the level-spacing distribution to exhibit characteristics of GUE statistics. This was numerically verified [115, 118] for both the Africa and one-eighth of the Sinai billiard subject to a uniform, perpendicular magnetic field $\left(\Phi=\Phi_{0} / 8000\right)$.

For a stronger magnetic field (e.g., $\Phi=\Phi_{0} / 800$ ), Landau levels become important, especially for energy close to the Dirac point where the density of states is low. The energy levels are then clustered, leading to $\partial N / \partial E \rightarrow \infty$ at the Landau levels. The staircase counting function deviates markedly from the semiclassical predictions. The level-spacing statistics for this case are not physically meaningful. Nonetheless, if one proceeds with the same calculation and analysis, the statistical quantities $P(S)$ and $I(S)$ would exhibit apparent deviations from the GUE statistics, and in fact they are closer to GOE behaviors. Intuitively, this can be understood by noting that the Landau levels "squeeze" the energy levels around them, resulting in smaller level-spacings and larger values of $P(S)$ for small $S$. Since the overall slope of the staircase counting function is unchanged, squeezing of the energy levels around the Landau levels tends to stretch the energy levels in between the different Landau levels, yielding large spacings and consequently larger values of $P(S)$ for large $S$. The stretching process causes a gradual transition of the level-spacing distribution from GUE to GOE. Similar results were observed [115, 118] for nonrelativistic quantum chaotic billiards where the system is described by the Schrödinger equation, in the energy range 
where the density of states is low and Landau levels arise. The spectral rigidity, however, does not fall into any of the three known categories. This is due to the fact that the staircase function no longer follows the semiclassical prediction when the effects of Landau levels cannot be neglected in the presence of a strong magnetic field.

For higher energy levels, e.g. $0.4<E / t<0.7$, where the Landau levels are not apparent, the level-spacing statistics return to the GUE class. In this energy range, trigonal warping becomes dominant (Fig. 12), rendering the theoretical description of Dirac equation inappropriate. The energy levels can be accessed in experiments and thus are relevant to transport through graphene quantum dot systems. In this region, $N\left(E_{n}\right)$ still depends hyperbolically on $E_{n}$, indicating a linear dependence between $E_{n}$ and $k_{n}$. This is because, in this energy range, the trigonal warping determines the pointer states (density patterns obtained from eigenstates) in the system and restrains them to having line segments only in three directions, e.g., from the Dirac points to the origin [121]. Along these directions, the $E-k$ relation is approximately linear up to $E / t \sim 1$. This explains the hyperbolic relation between $N\left(E_{n}\right)$ and $E_{n}$ even for high energies far away from the Dirac point.

The emergence of the GUE statistics for higher eigenenergies corroborates the argument that about the Dirac point, the energy level-spacing statistics are shifted "artificially" to GOE by the Landau levels. This could be experimentally relevant as the level spacings can possibly be revealed in the peak spacings in the conductance of the corresponding quantum dots made from "open" billiards [294].

Classically, the effects of a magnetic field on chaos were studied quite early [295, 296], where it was found that for billiards with sufficiently smooth boundaries, such as an integrable elliptical billiard, flyaway chaos can be induced by a magnetic field, but increasing the field strength to the Landau regime can remove chaos.

\section{Level spacing statistics for edge states in chaotic graphene billiards}

A prominent feature of graphene billiards is the emergence of a large number of edge states. Characteristics of the level-spacing statistics of the edge states were studied [116] for a graphene quantum dot that has the shape of a deformed disk with an angle dependent radius function: $R(\theta)=R_{0}+0.2 R_{0} \sin (\theta)+0.05 R_{0} \sin (2 \theta)-0.025 R_{0} \sin (3 \theta)+0.02 R_{0} \sin (4 \theta)-0.01 R_{0} \sin (5 \theta)$, with $R_{0}=160 a \approx 40 \mathrm{~nm}$. In order to distinguish the edge from the bulk states, one can calculate the participation ratio [297, 298] for each eigenstate $\psi$, which is the fraction of atoms occupied by an electron for a given energy level,

$$
p=\frac{\left(\sum_{i}|\psi(i)|^{2}\right)^{2}}{N \sum_{i}|\psi(i)|^{4}},
$$

where the index $i$ runs over the atomic sites and $N$ denotes the total number of atoms in the dot. For extended states, one has $p \sim 1$ but for localized edge states, the values of the participation ratio are at least two orders of magnitude smaller: $p \approx 10^{-4}-10^{-2}$.

Because of their localized behavior, the level spacings associated with the edge states are expected to follow the Poisson statistics [299]. Surprisingly, as shown in Fig. 16, the level-spacing distribution for the electron-hole symmetric case $t^{\prime}=0$ follows the GOE statistics. Poisson statistics arise when the electron-hole symmetry is broken, e.g., for $t^{\prime}=0.1 t$. 


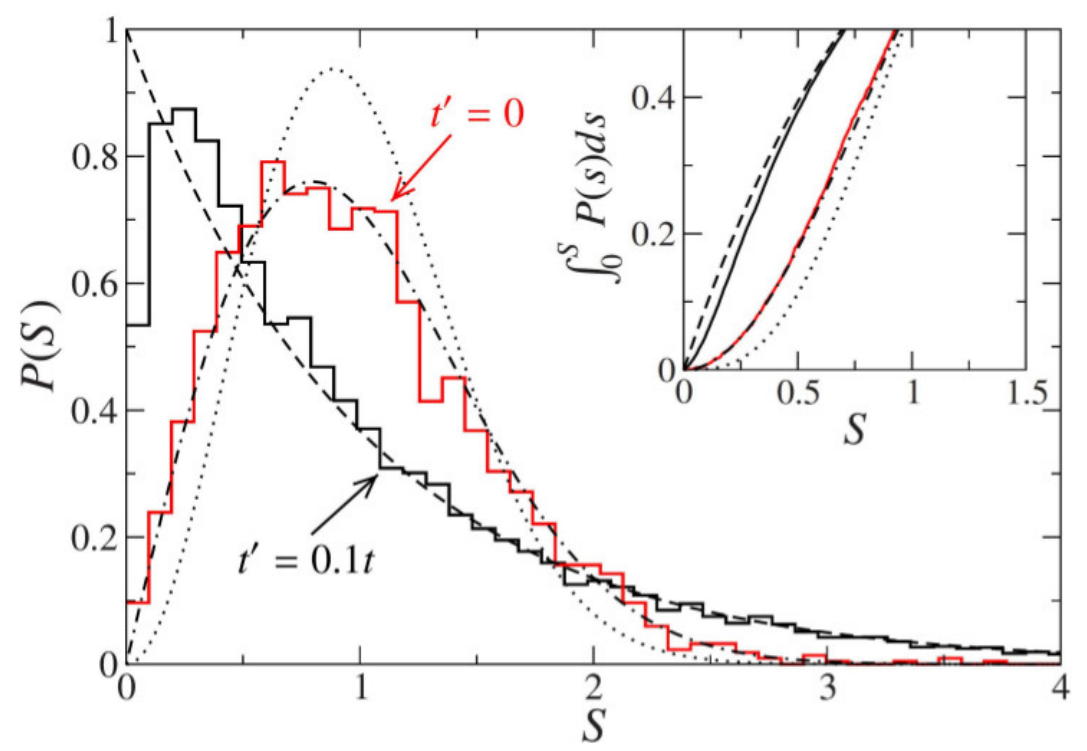

FIG. 16. Level-spacing distributions for edge states in graphene quantum dots. The solid red and black curves are for cases where there is an electron-hole symmetry $\left(t^{\prime}=0\right)$ and the symmetry is broken $\left(t^{\prime}=0.1 t\right)$, respectively. The dashed, dashed-dotted, and dotted curves correspond to the distributions associated with Poisson, GOE, and GUE statistics, respectively. The inset shows in detail the integrated level-spacing distributions for small level spacing $S$. The statistics were obtained using an ensemble of 100 quantum dots. From Ref. [116] with permission.

The striking difference between the level-spacing statistics of edge states and those of the bulk states can be understood by examining the peculiar features of the wavefunctions associated with the edge states. The graphene Hamiltonian has a chiral symmetry for $t^{\prime}=0$ that results in an equal occupation probability of sublattices A and B for every individual wavefunction [292]. Since the wavefunction at a certain type of zigzag edge is nonzero only on one sublattice, every eigenstate for $t^{\prime}=0$ must also occupy another part of the boundary of the opposite kind, as illustrated in Fig. 17. This leads to an artificial long-range coupling among the edge states and thus to level repulsion, resulting ultimately in GOE statistics. If the chiral symmetry is broken, e.g., by nonzero secondnearest-neighbor hopping, edge-state wavefunctions can be localized at a single edge. Figure 17 shows that, close to a boundary, the edge states occupy a single sublattice only. In order to avoid the oscillatory pattern on the lattice scale that inevitably arises when both sublattices are taken into account simultaneously, for every unit cell only the atom with the largest occupation probability is plotted.

\section{Can valley decoupling lead to GUE statistics in graphene billiards without any magnetic field?}

Since graphene has quasiparticles that mimic relativistic, massless Dirac fermions, it seems plausible to test the prediction of Berry and Mondragon about the GUE statistics of Dirac particles [93] with classically chaotic graphene confinement systems. A complication is that graphene has two valleys, so the two massless Dirac fermions, each originating from a valley, are coupled through mechanisms such as abrupt edge cuts or short range disorders. The whole system, which includes the confinement and the interaction between the two quasiparticles, in fact pre- 


$$
t^{\prime}=0 \quad t^{\prime}=0.1 t
$$

a)
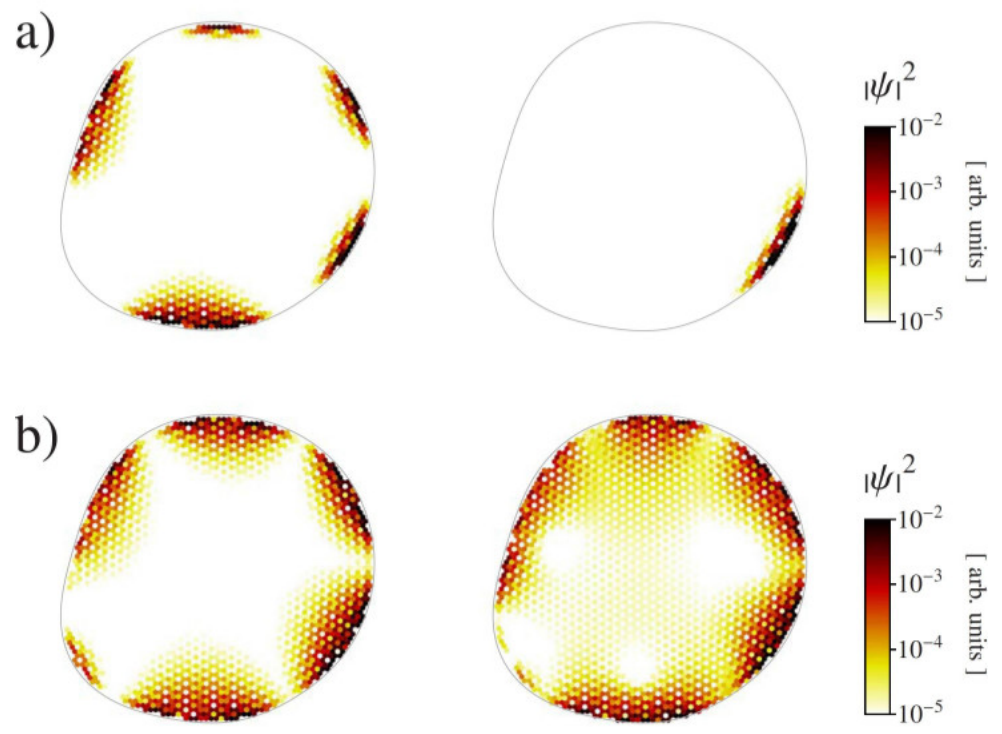

FIG. 17. Wavefunction density in a graphene quantum dot. The left column is where the electron-hole (e-h) symmetry is preserved $\left(t^{\prime}=0\right)$, while the right column is for the case where this symmetry is broken $\left(t^{\prime}=0.1 t\right)$. The examples are for an edge mode that decays quickly (a) and slowly (b) into the bulk. The nominal radius of the quantum dot is $R_{0}=30 a$. From Ref. [116] with permission.

serves the time-reversal symmetry, leading to GOE level-spacing statistics, as demonstrated in Sec. III D 3. Intuitively, if the two quasiparticles can be decoupled so that their motions become independent, with a finite mass confinement, GUE statistics would emerge. This conjecture was investigated through a smoothly varying mass confinement [299], where the mass term can be realized through an effective staggered potential caused by possible edge magnetization of graphene flakes [300, 301]. The domain of an Africa billiard was studied [299], which contains 68169 carbon atoms, resulting in about 3000 energy levels in the range $[-0.5 t, 0.5 t]$. The mass term is given by $\sum m_{i}|i\rangle\langle i|$, which is positive (negative) if $i$ belongs to sublattice $A(B)$. For smooth mass confinement, the term is zero in the interior but finite within the distance $W=4.5 \sqrt{3} a$ of the boundary: it starts from zero at the inner border of the region and increases quadratically: $m_{i}=b^{2}\left[d_{i}-W\right]^{2} / 2$, where $d_{i}$ is the distance of atom $i$ to the boundary and $b=0.15 \sqrt{t} / a$ is a constant. While the lattice eventually terminates, for the smooth varying confinement, the rough boundary has little effect on the quasiparticle motion. As a result, inter-valley scattering is suppressed, which preserves the valley symmetry but breaks the sublattice symmetry as well as the time-reversal symmetry associated with each valley [299].

When the two valleys are decoupled (zero or weak inter-valley scattering), the Hamiltonian consists of two degenerate blocks, each corresponding to a quasiparticle with unitary symmetry. As Fig. 18 shows, in spite of the efforts, the conjectured GUE statistics were never observed in the absence of a magnetic field. A plausible explanation is that, while the mass term is relatively smooth, residual inter-valley scattering can persist. Even for arbitrarily weak inter-valley interactions, the scattering time can be shorter than the relevant Heisenberg time scale for level spacing, leading to GOE statistics [299]. The symmetry properties [266, 302-304] are also related to the pseudospin effects in graphene, which can significantly suppress backscattering [305, 306] 


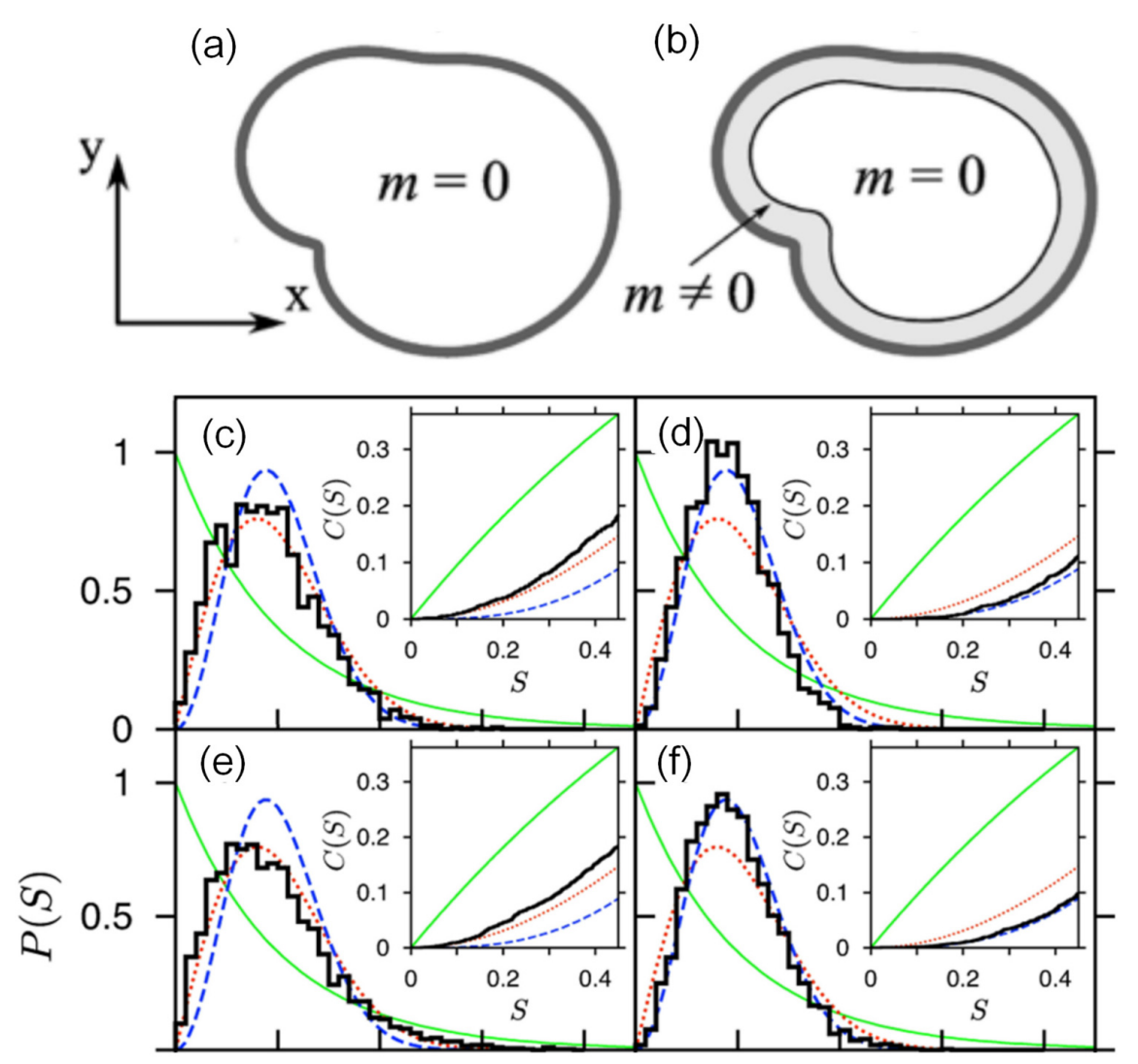

FIG. 18. Level-spacing distributions for Africa shaped graphene billiard with smooth mass confinement. (a,b) Africa shaped graphene billiard with abrupt edge termination and smooth mass confinement, respectively. (c-f) Level-spacing distribution for a billiard with 68169 carbon atoms using about 3000 energy levels in the range $[-0.5 t, 0.5 t]$, and insets show the integrated distribution. (c,d) Abrupt lattice termination. (e,f) Smooth mass confinement with $W=4.5 \sqrt{3} a$. Panels (c) and (e) are for cases without a magnetic field, while (d) and (f) are for $\Phi=0.7 \Phi_{0}$. From Ref. [299] with permission.

and effectively eliminate weak localization or anti-localization [293, 307-311]. This can lead to conductance fluctuations that belong to different universality classes [299, 312].

The level-spacing statistics of highly symmetric (triangular and hexagonal) graphene nanoflakes with armchair, zigzag, and Klein [117, 313] boundaries were also studied [119]. The main advantage of using such geometric shapes is that the boundaries are "pure" in the sense that they are of only one type (either armchair, zigzag, or Klein) and there is no mixing of different types. In the absence of any random disorder, due to the geometric symmetry, the energy levels are clustered and exhibit Poisson like behaviors, although the level-spacings do not rigorously fit within any class of random matrix ensembles. When disorders such as substrate impurities or ions [314, 315] are present, a transition in the level-spacing statistics from Poisson-like to GOE statistics occurs. Numerical results [119] indicate that the orthogonal symmetry emerges generically in such closed graphene nanosystems. In the special case of a triangular nanoflake with zigzag (or Klein) edges and smooth impurity potentials, the unitary symmetry class is the relevant one. A subsequent work [316] investigated the effects of strain fields that can be regarded as a strain-induced gauge field, with the finding that if the zigzag triangular nanoflake is deformed such that all its geometric 
symmetries are broken, the spectral statistics are of the GUE type. However, if a mirror symmetry is present, the spectral statistics belong to the GOE class.

\section{Peculiar level-spacing statistics in circular sector graphene billiards}

The fluctuation properties of the eigenvalue spectra of finite-size graphene billiards with the geometric shape of circular sectors of angles $\pi / n$, where $n$ is an integer, turn out to exhibit unconventional features [120]. Such a billiard domain can be cut from a perfect graphene sheet, and the underlying classical dynamics are integrable. The orientation of the domain can be chosen such that one of the straight boundaries of the sector are of the zigzag type. In a recent work [120], the Hamiltonian matrices corresponding to lattice sizes of up to $2 \times 10^{5}$ atoms were diagonalized. Table II lists the lattice structures studied.

TABLE II. Graphene sector billiards. The letter " $N$ " denotes the non-perfect cases where a few atoms at the corner or one row of atoms at one of the straight boundary are missing, i.e., their shapes slightly deviated from that of a sector billiard. The letters " $\mathrm{Z}$ " and " $\mathrm{A}$ " stand for zigzag and armchair edges along the straight boundaries, respectively. The size of the graphene sheet is specified by the number of lattice sites.

\begin{tabular}{lcccc}
\hline \hline Angle & $15^{\circ}$ & $45^{\circ}$ & $30^{\circ}$ & $30^{\circ} \mathrm{N}$ \\
\hline Size & 226993 & 244664 & 220862 & 221944 \\
\hline Angle & $90^{\circ}$ & & & \\
\hline Size & 217615 & $60^{\circ} \mathrm{Z}$ & $60^{\circ} \mathrm{A}$ & $60^{\circ} \mathrm{AN}$ \\
\hline \hline
\end{tabular}

Altogether, as listed in Table II, eight sectors with varying edge structures along the straight boundaries and angles $\pi / n$ with $n=2,3,4,6,12$ were considered [120], which correspond to $15^{\circ}, 30^{\circ}, 45^{\circ}, 60^{\circ}$, and $90^{\circ}$, respectively, whereas the curved boundary was obtained by fitting the hexagonal lattice into the corresponding sector billiard. For the $15^{\circ}$ and $45^{\circ}$ sectors, one straight boundary is terminated by a zigzag edge, while the other one consists of a mixture of zigzag and armchair segments. For the $30^{\circ}$ and $90^{\circ}$ domains, a perfect cut results in a zigzag edge for one straight boundary and an armchair edge for the other, so there is no reflection symmetry. For the $30^{\circ}$ sector, besides perfect cut, non-perfect cut was also studied, with the only difference being one additional layer of atoms along the armchair edge. A $60^{\circ}$-sector graphene billiard can be cut out of a hexagonal lattice such that both straight boundaries are zigzag or armchair edges. Accordingly, these graphene billiards can possess an exact reflection symmetry. In addition, a nonperfect $60^{\circ}$ sector structure was considered [120] in which the reflection symmetry was broken by removing one atomic layer along one of the straight boundaries. Taking into account only the nearest-neighbor hopping term in the Hamiltonian yields eigenstates that are symmetric under the $E \rightarrow-E$ and the $\left[\Psi_{A}, \Psi_{B}\right] \rightarrow\left[\Psi_{A},-\Psi_{B}\right]$ operations [292]. It thus suffices to consider only the states corresponding to positive energy values, $0 \lesssim E / t \leq 3$.

The eigenstates of a nonrelativistic quantum billiard in a sector domain [317] of unit radius and arbitrary angle $\varphi_{0}$, i.e., of a quantum particle trapped in an infinitely high potential well with the shape of a sector, can be obtained by solving the Schrödinger equation with the Dirichlet boundary conditions along the border of the domain. They are given in terms of the Bessel func- 
tions, $\Psi_{m n} \propto \sin \left(m \pi \varphi / \varphi_{0}\right) J_{m \pi / \varphi_{0}}\left(k_{m n} \rho\right)$, where $\varphi$ and $\rho$ are the angular and radial polar coordinates, respectively, and the indices are integers: $m, n=1,2,3, \cdots$. The eigenwavevectors $k_{m n}>0$ can be obtained from the zeros of the Bessel functions along the boundary, i.e., for $\rho=1$, $J_{m \pi / \varphi_{0}}\left(k_{m n}\right)=0$, which yields the eigenenergies $E_{m n}=\hbar^{2} k_{m n}^{2} /(2 m)$, where $m$ is the mass. For large values of $x$, one has

$$
J_{m \pi / \varphi_{0}}(x) \sim \sqrt{2 /(\pi x)} \cos \left[x-\left(m \pi / \varphi_{0}\right) \pi / 2-\pi / 4\right],
$$

so, asymptotically, the zeros for different $m$ values are shifted by integer multiples of $\pi^{2} / 2 \varphi_{0}$ with respect to each other [10]. In nonrelativistic quantum mechanics, the spectral statistics of the sector billiards were shown to be of the Poisson type [10]. The intuitive expectation is then that the eigenvalues of sector-shaped graphene billiards should exhibit a similar behavior, regardless of the value of $\varphi_{0}$. However, it was recently discovered [120] that this was not the case and the type of level-spacing statistics in fact depends on the energy range under consideration.
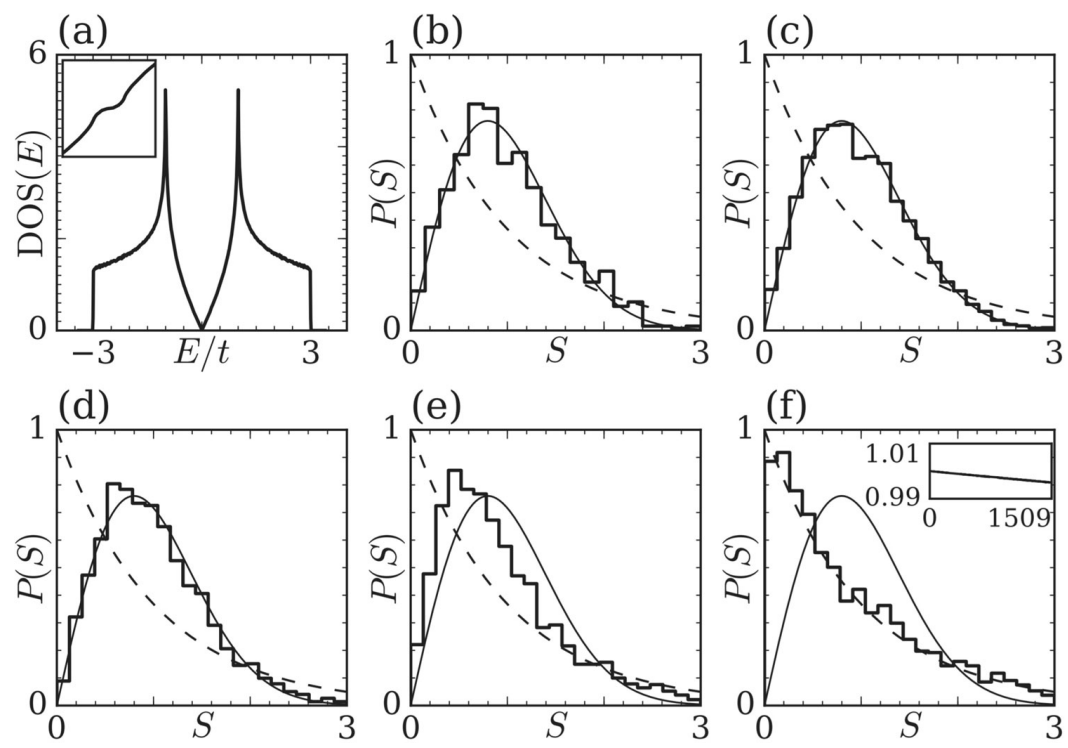

FIG. 19. Spectral properties of a graphene billiard with the shape of a $15^{\circ}$ sector. (a) Density of states. Inset: integrated density of states. (b-f) Nearest-neighbor spacing distributions in the energy ranges: (b) $E / t \in[0.02,0.2], 837$ levels; (c) $E / t \in[0.5,0.6], 2575$ levels; (d) $E / t \in[2.0,2.1], 3804$ levels; (e) $E / t \in[2.6,2.7], 3321$ levels; (f) $E / t \in[2.95,3], 1509$ levels. The histograms show the numerical results, while the dashed and solid lines indicate the Poisson and GOE distributions, respectively. Inset in panel (f): normalized ratio $\left(E-E_{\text {edge }}\right) / E_{Q B}$ for the first 1509 eigenenergies, where $E_{Q B}$ is the accurate eigenenergy obtained from analytical solutions of the corresponding quantum billiard. From Ref. [120] with permission.

The findings [120] can be summarized, as follows. Near the band edges $E / t= \pm 3$, the dispersion relation is parabolic, e.g., $E-E_{\text {edge }} \sim k^{2}$, thus the graphene pseudo-particles are essentially described by the nonrelativistic Schrödinger equation. Indeed, both the eigenenergies and eigenwavefunctions [Figs. 20(a-d)] agree with the analytical solutions well. The level-spacing statistics are thus Poisson, as shown in Fig. 19(f). As the energy deviates from the band edge, e.g., for $E / t \in(2.6,2.7)$, the spectral statistics evolve toward the GOE type, as shown in Fig. 19(e). In this energy range, the structure of the boundary, which is not perfectly smooth, leads to spectral statistics that are not purely Poisson, but to those that lie somewhere between the Poisson and 


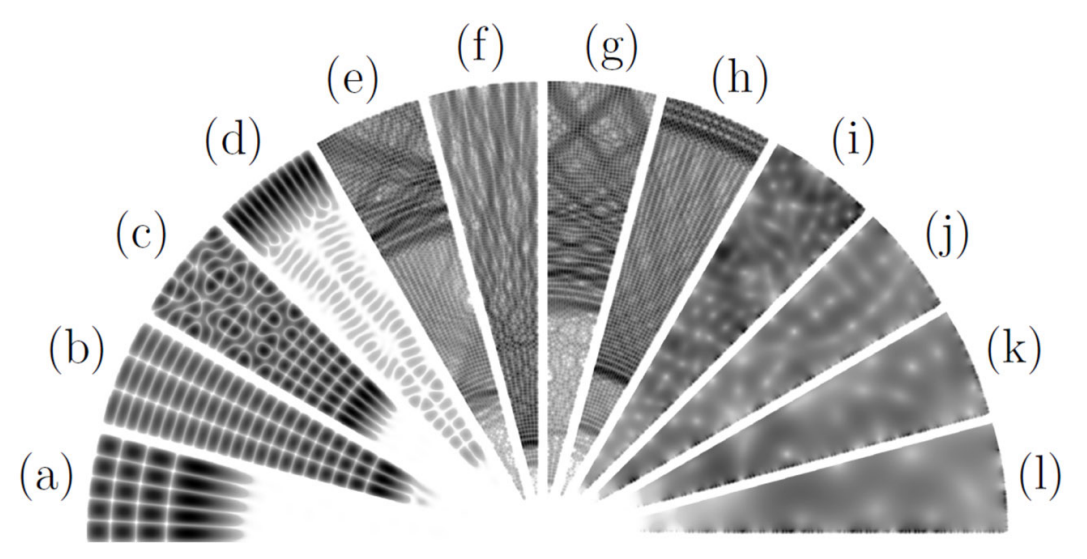

FIG. 20. Intensity distributions $|\Psi|^{2}$ in the $15^{\circ}$-sector graphene billiard. Panels (a-1) correspond to energy values $2.991,2.981,2.976,2.974,2.667,2.664,2.663,2.661,0.195,0.140,0.100$, and 0.063 , respectively. In panels (a-d), the energies are close to the band edge, corresponding to eigenstates with $(m, n)=(5,4),(3,22),(5,18)$, and $(11,1)$ in the quantum billiard of corresponding shape, respectively. Panels (e-h) show results for the transitional region around $E / t \sim 2.66$. In panels (i-1) the energies are close to the Dirac point. From Ref. [120] with permission.

GOE type. Certain patterns of the intensity distributions of the eigenstates are similar to those of the squared wavefunctions of the corresponding nonrelativistic quantum billiard. However, there are nonideal factors in the wavefunctions in comparison with the analytical solutions, as shown in Figs. 20(e-h). As the energy deviates further from the band edge, e.g., for $E / t \in[2.0,2.1]$, the spectral properties exhibit an increasing similarity to the GOE statistics, as shown in Fig. 19(d). In this energy range, while the eigenfunctions may retain certain features of the wavefunctions of the corresponding nonrelativistic quantum billiard, the pattern structure can be quite different. These features are reminiscent of the fluctuation properties in the eigenvalue spectra of nonrelativistic quantum billiards with mixed Dirichlet and Neumann boundary conditions [318], where in the present case the boundary conditions might not be purely Neumann or Dirichlet. Note that the mixed boundary conditions are a purely wave-dynamical feature with no classical correspondence.

For energy values close to the Dirac point, excluding the edge states, the effective wavelengths are long, so one may expect that the spatially discrete nature of the boundaries will have little effect on the statistical properties of the eigenstates and, as such, the fluctuations in the energy spectra should be describable by Poisson statistics. However, the occurrence of the Dirac point has its origin in the two inter-penetrating triangular sublattices forming the hexagonal lattice of graphene. Computations revealed [120] that the spectral properties agree well with those associated with GOE statistics [Fig. 19(b)]. While surprising, the phenomenon can be qualitatively understood from the properties of the intensity distributions, which are typically disordered patterns spread over the entire billiard plane [Fig. 20(i-1)]. Furthermore, the intensities are peaked at some parts of the zigzag edges of the straight boundary formed by a mixture of armchair and zigzag edges, similar to the squared wavefunctions in a nonrelativistic quantum billiard with Neumann conditions at these parts of the boundary. Varying the boundary conditions leads to correlations in the wave amplitudes at the sites of the corresponding sublattice and, through hopping, between the sublattices. As a result, level repulsion arises, which is typical for graphene sector billiards.

In general, when the lattice structure of the graphene billiard has a higher symmetry includ- 
(a)

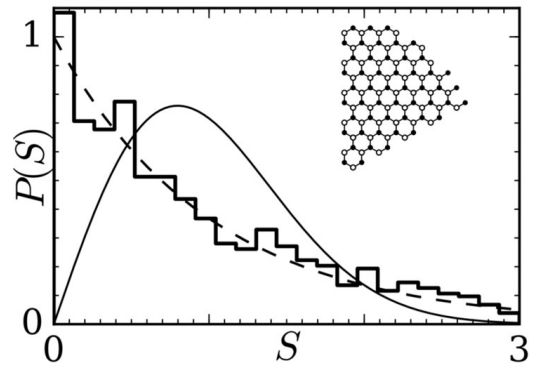

(b)

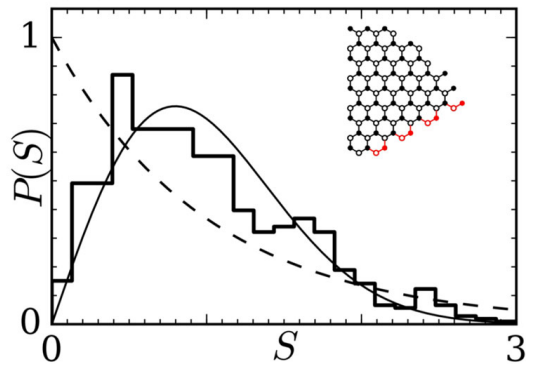

FIG. 21. Effect of structural perturbation on the nearest-neighbor spacing distribution for a graphene billiard with the shape of a $60^{\circ}$ sector. (a) armchair edges, (b) armchair edges with one row of atoms added along one edge. The energy range is $E / t \in[0.02,0.2]$. Insets: magnified view of the lattice structure close to the tip of the sector to illustrate the differences. Adapted from Ref. [120] with permission.

ing uniform boundary conditions for each sublattice along its perimeter, as for graphene billiards with the shape of an equilateral triangle [119] or a rectangular billiard [319], the patterns of the intensity distributions have an ordered (periodic) structure and the spectral properties tend to be more Poisson-like. This is also the case for the sector billiard. For example, for the $60^{\circ}$ sector billiard with perfect armchair boundaries, the level-spacing statistics are Poisson, as shown in Fig. 21(a). However, a small variation in the boundary conditions, e.g., by adding just one row of atoms [inset of Fig. 21(b)], or alternatively, by removing a few atoms around the tip, may lead to a drastic change in the eigenstates, giving rise to GOE statistics, as shown in Fig. 21(b). This extreme sensitivity on the structure of the boundary arises but only for energy values close to the Dirac point. Nevertheless, such small perturbations to the boundary will not affect the nature of the spectral properties in the low-energy region for the corresponding quantum billiard, as the eigenenergies and eigenwavefunctions of the same graphene sector billiard close to the band edge agree with the analytical solutions very well. However, a point-like perturbation, a change in the boundary at a singular point, will change dramatically the wavefunctions and hence the spectral properties [320, 321].

The distributions of the moduli of the wavefunction components on the lattice sites were also studied [120] with the finding that they are well described by a Gaussian function typical for chaotic systems in energy regions where the spectral properties are described by GOE statistics. Near the Dirac point, states localized along the zigzag rows and the van Hove singularities [227] must be excluded.

\section{E. Microwave Dirac billiards}

The microwave Dirac billiards can be constructed from photonic crystals composed of metallic cylinders and arranged on a triangular lattice, which were squeezed into a cylindrical microwave cavity with a much smaller height in the $z$ direction compared to those in the $(x, y)$ plane. Below a certain frequency that is inversely proportional to the height of the cavity, the underlying Helmholtz equation is two dimensional and mathematically equivalent to the Schrödinger equation of the corresponding quantum billiard. The shape of the microwave cavity in the $(x, y)$ plane can be constructed arbitrarily, which can simulate 2D quantum billiards with either integrable or 
(a)

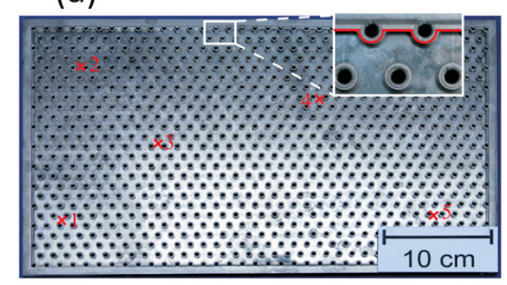

(d)

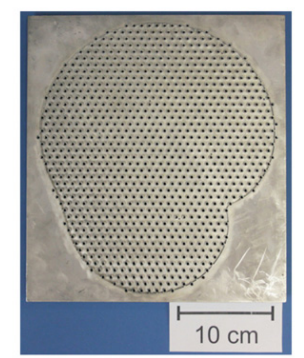

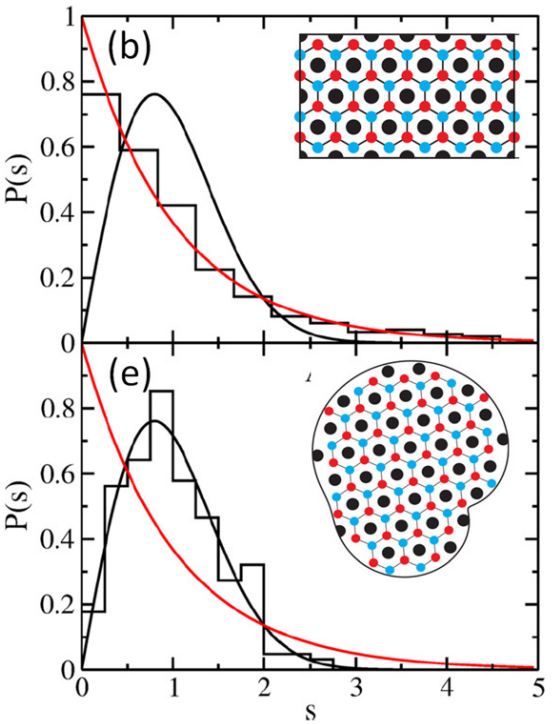

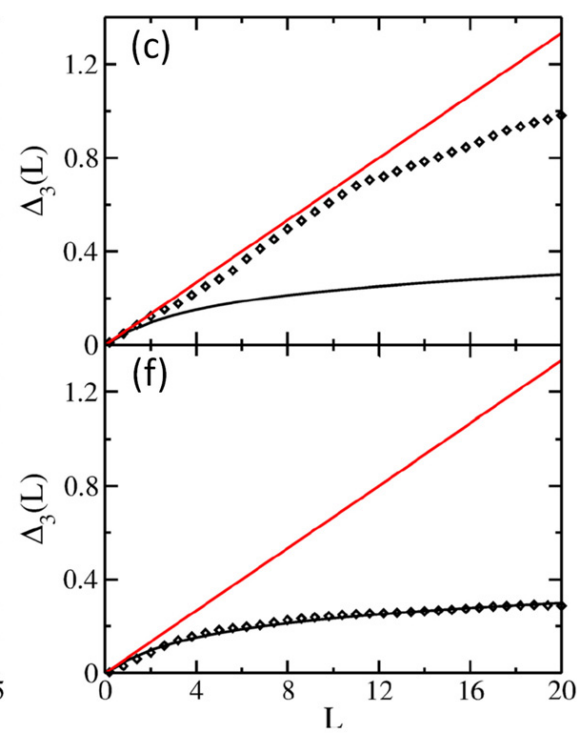

FIG. 22. Level spacing statistics for microwave Dirac billiards. Upper and lower panels are for the rectangular and chaotic Africa shaped billiard, respectively. Left panels show the Dirac billiard that contains about 900 metallic cylinders arranged on a triangular lattice, middle panels are for the nearest-neighbor spacing distributions, where insets show the honeycomb lattice structure of the voids between the metallic cylinders, and right panels show the spectral rigidity of the resonance frequencies close to the Dirac point obtained from the microwave Dirac billiards under superconducting condition. Adapted from Ref. [224, 225] with permission.

chaotic classical dynamics. Due to the cylinders, the electromagnetic wave propagates along the voids between them, forming a honeycomb lattice, and the frequencies as a function of the two quasi-momentum components exhibit a band structure similar to that of graphene. Accordingly, the resonant spectra of the microwave billiard systems contain Dirac points governed by the relativistic Dirac equation. Such experiments can be performed at the room temperature, but the typical quality factor is about $10^{3}$, so the fine structures in the transmission or reflection spectra cannot be identified. The Darmstadt group [225] carried out such measurements under the superconducting condition, for which the quality factor can be as high as $10^{7}$, making it possible to determine sequences of thousands of eigenvalues. Accurate energy level statistics can then be obtained.

Figure 22 shows the level-spacing statistics of two microwave Dirac billiards with classically integrable and chaotic dynamics, respectively. The resonant spectra were obtained under the superconducting condition. From the nearest-neighbor spacing distributions and the spectral rigidity, one can see that the rectangle microwave Dirac billiard exhibits Poisson statistics, while the Africa shaped microwave billiard exhibits GOE statistics [224, 225], in agreement with those of graphene billiards [115, 118, 286].

As in a graphene sheet or, generally, in artificial graphene, the smooth part of the density of states of the Dirac billiards has a complicated structure and exhibits sharp peaks at the van Hove singularities. There, the electronic properties of graphene and, likewise, the wave function structures calculated from the tight-binding model, change rapidly with increasing frequency. Nevertheless, it was demonstrated that [227], the spectral properties of the Dirac billiards are de- 
termined only by their shapes. For example, the rectangular microwave Dirac billiard gives the Poisson statistics, while the Africa shaped billiard generates the GOE statistics. In the vicinities of the van Hove singularities, the commonly used statistical measures are not applicable. An alternative is to use the ratio or the overlapping ratio distribution, which can provide useful statistical measures of the spectral properties.

\section{F. Concluding remarks on energy level-spacing statistics}

The seminal work of Berry and Mondragon [93] was perhaps the first to treat the energy levelspacing statistics of classically chaotic billiards in the relativistic quantum realm. Their finding was that, for 2D massless Dirac particle confined in a finite region, time-reversal symmetry is broken due to the infinite-mass confinement potential, leading to GUE statistics even without any magnetic field. Graphene has low energy quasiparticles mimicking the 2D massless Dirac particle when inter-valley scattering is neglected. For an infinite graphene flake without disorder, the system preserves the pseudospin symmetry (the symmetry between A and B atoms) and also the symmetry between the two valleys. However, for a finite graphene billiard, the two symmetries are typically broken. The two valleys (or two quasiparticles) are then coupled, leading to GOE statistics.

The profound connection between the symmetries of the system and the random matrix ensembles for level-spacing statistics lies in the matrix representation of the Hamiltonian [3]. For example, for a system that preserves the time-reversal symmetry but with spin neglected, it is invariant under orthogonal transformations, and there are general representations other than the energy basis in which all the elements of the matrix are real. Since the Hamiltonian is Hermitian, it must be real symmetric. Classical chaotic dynamics will introduce randomness into the real symmetric Hamiltonian matrix, leading to GOE statistics. Intrinsic disorders and edge roughness will have the same effect of introducing randomness to the Hamiltonian matrix. If the time-reversal symmetry is broken and there is no other symmetry that can be preserved together, the system will be invariant under unitary transformations, and the elements of the Hamiltonian matrix in a general representation will not all be real. Due to disorders, the matrix will be a random Hermitian matrix with complex elements. The resulting energy level statistics will then be of the GUE type. For graphene described by the tight-binding Hamiltonian, short-range or long-range disorders, strain, and mass term make the Hamiltonian matrix random but it remains to be real symmetric, so in general GOE statistics will emerge. One exception is that, even for systems with time-reversal symmetry, certain subset of eigenstates associated with some discrete symmetry of the system can be identified [259, 322], for which the level-spacing statistics can be GUE. 


\section{RELATIVISTIC QUANTUM SCARS}

\section{A. Quantum scars - an introduction}

A remarkable phenomenon in quantum systems whose dynamics in the classical limit are chaotic is scarring. In particular, in the semiclassical regime a wavefunction can be regarded locally as a superposition of many plane waves. Due to classical chaos, the directions of these plane waves are uniformly distributed. Intuitively, one may expect the wave functions to have a uniform concentration in the position space. However, signatures of highly non-uniform distribution of the wave function were discovered by McDonald and Kaufman in their study of the Helmholtz equation in the classically chaotic stadium billiard [26]. Numerical investigations of the system in the semiclassical regime by Heller [28] revealed the striking "scarring" phenomenon that the wavefunctions tend to concentrate on paths corresponding to unstable periodic orbits in the classical limit. Theoretical explanations of the scarring phenomenon were given by Bogomolny [53] and Berry [1] based on the semiclassical Green's function. Since then, quantum scars have become an active topic of research [27, 29, 52, 54-79] in the traditional field of nonrelativistic quantum chaos [2, 152] for systems described by the Schrödinger equation.

While quantum scars typically arise in closed Hamiltonian systems, in open systems (e.g., quantum dots) the counterparts of scars exist: quantum pointer states [73, 155-160], which can have a significant effect on the transport properties $[69,80,81,161,162]$ such as inducing conductance fluctuations. Especially, pointer states associated with more pronounced scars in the corresponding closed system can "trap" electrons longer in the quantum dot, leading to narrower conductance resonances. In fact, from the properties of scars in the closed system, one can infer the extent of conductance fluctuations in the corresponding open system.

In the development of RQC, two questions concerning scarring naturally arose: (1) can there be scars in relativistic quantum systems? and if yes, (2) can there be relativistic quantum scars that have no counterparts in nonrelativistic quantum systems?

The first question was partly addressed [121] through a study of chaotic graphene billiards described by the tight-binding Hamiltonian, where scars were found. However, graphene is a discrete-lattice system with two non-equivalent Dirac points in the energy-momentum space. Although the electronic behavior in the neighborhood of each Dirac point can be described by the massless Dirac equation [101-107], physical processes such as reflection from the system boundaries can couple the quantum dynamics from the two Dirac points. Thus, in a strict sense the underlying physics in graphene is not exactly that given by the single particle Dirac equation, but is always that of coupled particles from the two distinct Dirac points. The scars in chaotic graphene confinements [121] are thus relativistic quantum scars only in an approximate sense.

It has been mentioned in Sec. II that, with respect to the general issue of relativistic quantum manifestations of classical chaos, Berry and Mondragon [93] developed a boundary-integral method to solve the massless Dirac equation (for neutrino) in closed domains such as that given by the chaotic Africa billiard. While they provided an integral expression for calculating the eigenstates, the focus of the study [93] was on energy-level statistics.

The second question was addressed in Ref. [123], in which the authors employed a conformalmapping based method to solve the Dirac equation in closed chaotic domains confined by an infinite mass potential. In particular, the boundary of certain chaotic domain can be generated from the unit circle through a conformal mapping, so the analytic solutions of the eigenvalues 
and eigenstates of the Dirac equation in the circular domain can be used to determine those in the chaotic domain. This has led to the success of accurately determining a large number of energy levels and the associated eigenstates. Examination of the eigenstates revealed a class of relativistic quantum scars in massless Dirac fermion systems: chiral scars that occur on classical periodic orbits with odd number of bounces off the domain boundary. For a chiral scar, the phase difference of a full rotation about the periodic orbit and its time reversed counterpart will gain an additional $\pi$, leading to anomalous half unity values of a characteristic quantity from the conventional semiclassical theory. There are thus no counterparts of chiral scars in nonrelativistic quantum systems because, there, this semiclassical quantity can take on values of either zero or one.

In this Section, we first discuss quantum scars in chaotic graphene billiard systems. We then turn to chiral scars, focusing on its unique properties, the fundamental physical mechanisms based on symmetry considerations, and controlled generation of those scars through an external magnetic field.

\section{B. Quantum scars in graphene billiards}

A convenient setting to investigate quantum scarring in chaotic graphene systems is stadiums. The tight-binding approximation allows the wave functions to be calculated both in the low-energy regime where the particle motions obey the Dirac equation and in the high-energy regime where trigonal warping distortion becomes dominant [271, 283, 323]. The calculations and analysis revealed [121] signatures of pronounced concentrations of eigen-wavefunctions about certain classical periodic orbits.

For a chaotic stadium graphene billiard, it is necessary to remove the boundary atoms with only one neighbor to avoid possible artificial scattering effects. Figures 23(a-c) show three typical patterns of electron concentration for three distinct energy levels. Since the system is relatively large, the $E-k$ relation for an infinite graphene flake can be used to gain insight. The allowed wavevectors for the specific energies of the patterns are shown in Figs. 23(d-f). Note that, for an infinite system, the allowed wavevectors constitute continuous curves in the contour plots of energy versus wavevector. For a finite confinement, the allowed wavevectors are discrete. It is useful to divide the energy range into three regimes: low, intermediate, and high, and to discuss the mechanisms of scarring in each energy regime separately.

Low-energy regime $(E / t \ll 1)$. In this regime, the possible wavevectors are restrained to the vicinity of the Dirac points, as indicated by the small solid circles in Fig. 23(d). In addition, the number of allowed wavevectors is small, so that any quantum superposition consists of only a few allowed plane waves. Along the directions perpendicular to those from the origin to the Dirac points, the wavelength $\lambda \sim 2 \pi / k_{\perp}$ is large, where $k_{\perp}$ is the perpendicular component of the allowed wavevector, i.e., the diameter of the small solid circles in Fig. 23(d). For two plane waves with close wavevectors, say $\boldsymbol{k}_{1}=\boldsymbol{K}+\boldsymbol{k}_{\perp} / 2$ and $\boldsymbol{k}_{2}=\boldsymbol{K}-\boldsymbol{k}_{\perp} / 2$, where $\boldsymbol{k}_{\perp} \perp \boldsymbol{K}$, the composed wave is given by

$$
e^{i \boldsymbol{k}_{1} \cdot \boldsymbol{x}+\phi_{1}}+e^{i \boldsymbol{k}_{2} \cdot \boldsymbol{x}+\phi_{2}}=2 \cos \left[\boldsymbol{k}_{\perp} \cdot \boldsymbol{x} / 2+\left(\phi_{1}-\phi_{2}\right) / 2\right] e^{\boldsymbol{K} \cdot \boldsymbol{x}+\left(\phi_{1}+\phi_{2}\right) / 2},
$$

which represents a propagating wave along the direction $\boldsymbol{K}$ modulated by a standing wave in the perpendicular direction. The anti-node is given by

$$
\cos \left[\boldsymbol{k}_{\perp} \cdot \boldsymbol{x} / 2+\left(\phi_{1}-\phi_{2}\right) / 2\right]=0
$$




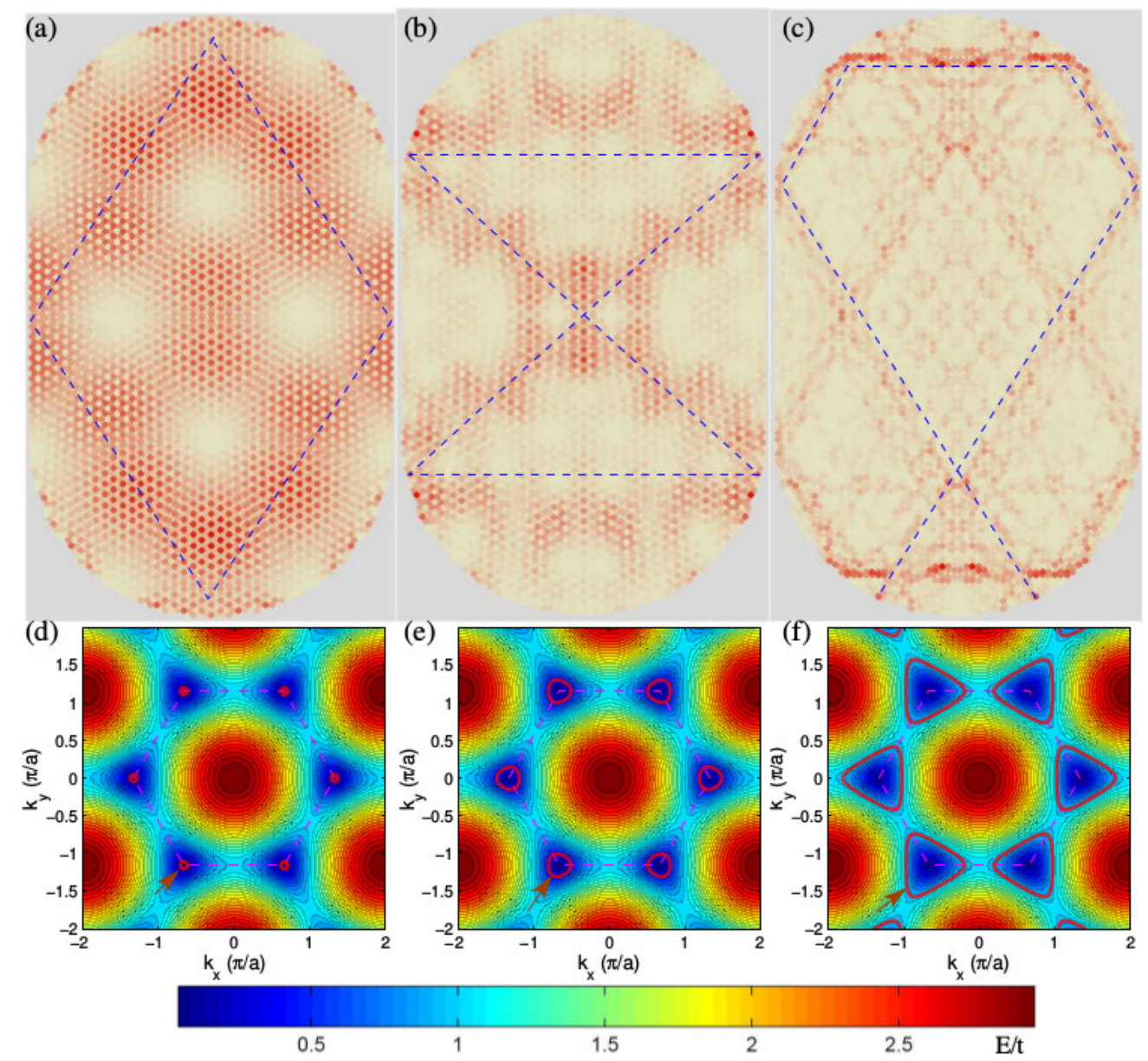

FIG. 23. Quantum scars in a chaotic graphene billiard of stadium geometry. Red color indicates higher wavefunction concentration associated with the scars. The stadium billiard contains $N=11814$ carbon atoms and has zigzag horizontal boundaries. The energy contour plots in the wavevector plane (the band structure) are also shown. The energies for the patterns in (a-c) are $E / t=0.13252,0.4024$, and 0.91188 , respectively. Panels (d-f) show the $E-\boldsymbol{k}$ configuration for an infinite graphene flake for the same lattice orientation and energy values as those for $(\mathrm{a}-\mathrm{c})$, respectively. The allowed wavevectors are on the constant energy curves (as indicated by the red arrows). Dashed line segments in (a-c) are for eye-guidance, and they indicate certain classical unstable periodic orbits. From Ref. [121] with permission.

so the distance between the nearby anti-nodes is $2 \pi /\left|\boldsymbol{k}_{\perp}\right|$. Since $|\boldsymbol{K}|$ is generally large (of the order of $\pi / a$ ), the wavelength along the $\boldsymbol{K}$ direction is small (extending a few lattice sites). Similar analysis holds for other allowed wavevectors [121]. For certain energy values, the superposition of the allowed plane waves forms standing waves and the anti-nodes of the standing waves (light region in the figure) have three directions only, which are parallel to the directions from the origin to the Dirac points. These features can be seen from Fig. 23(a).

Intermediate-energy regime $(E / t<1)$. In this regime, there are many allowed wavevectors that form nearly continuous contour lines (constant energy curves) in the wavevector space. As a result, the plane waves for each line segment of the allowed wavevectors generate a wave packet with group velocity determined by the gradient of the energy with respect to wavevector. The width of the wave packet is on the order of $\pi /|\Delta \boldsymbol{k}|$, where $|\Delta \boldsymbol{k}|$ is the width of the allowed wavevectors 
perpendicular to the direction of the group velocity. The contour lines are approximately circles, indicating that particle motion can take on any directions. As the linear relation between energy and wavevector holds in this energy range, the scars formed in this case are thus relativistic quantum scars. Since the group velocity can be in any direction, the orientations of the scarred patterns can be in any direction as well. The widths of the scars are typically large since $\Delta \boldsymbol{k}$ is still small about the Dirac points.

For slightly larger energy values, the constant energy curves acquire a nontrivial distortion, the so-called trigonal warping $[271,283,323]$. Although there is some tendency for the group velocity to focus on the three directions, as suggested by Fig. 23(e), the velocity can be in any direction, depending on the boundary conditions. Figure 23(b) provides such an example. Note that the diagonal line patterns are not along the directions from the origin to the Dirac points.

High-energy regime $(E / t \sim 1)$. In this regime, trigonal warping becomes more dominant, and the linear relation between energy and wavevector in the vicinity of the Dirac points, originally held in the low- and intermediate-energy regimes, is now violated, as shown in Fig. 23(f). The group velocity is proportional to the gradient $\nabla_{\boldsymbol{k}} E$, which is locally perpendicular to the constant energy curve. From Fig. 23(f), one can see that the group velocity focuses on only three directions, i.e., those from the origin to the Dirac points. Since the zigzag boundaries are along the horizontal direction, the phase angles associated with the directions are 0 (or $\pi), \pi / 3(4 \pi / 3)$, and $2 \pi / 3(5 \pi / 3)$, respectively.

To understand the "sharpness" of the scars (as compared with those in the low- and intermediateenergy regimes), one can consider the following situation. Assume the wavevector can take on values from a line segment $\left[\boldsymbol{k}_{1}, \boldsymbol{k}_{2}\right]$, and let $\boldsymbol{k}_{m}=\left(\boldsymbol{k}_{1}+\boldsymbol{k}_{2}\right) / 2$ be the mean value and $\Delta \boldsymbol{k}=\left(\boldsymbol{k}_{2}-\boldsymbol{k}_{1}\right)$ be the difference. If $N$ allowed wavevectors are distributed uniformly on the line segment, the superposition of the corresponding plane waves is

$$
\sum_{\boldsymbol{k}} e^{i \boldsymbol{k} \cdot \boldsymbol{x}} \times|\Delta \boldsymbol{k}| / N \approx \int_{\boldsymbol{k}_{1}}^{\boldsymbol{k}_{2}} e^{i \boldsymbol{k} \cdot \boldsymbol{x}} d \boldsymbol{k}=|\Delta \boldsymbol{k}| \frac{\sin (\Delta \boldsymbol{k} \cdot \mathbf{x} / 2)}{\Delta \boldsymbol{k} \cdot \boldsymbol{x} / 2} e^{i \boldsymbol{k}_{m} \cdot \boldsymbol{x}},
$$

which is in fact a propagating wave of wavevector $\boldsymbol{k}_{m}$ modulated by the $\sin u / u$ term, where $u=\Delta \boldsymbol{k} \cdot \boldsymbol{x} / 2$. The modulation factor causes the wave to focus on a narrow linear region of width $\lambda=4 \pi /|\Delta \boldsymbol{k}|$, which is perpendicular to $\Delta \boldsymbol{k}$. Since $|\Delta \boldsymbol{k}|$ for each constant energy line segment is of the order of $\pi / a, \lambda$ can be as small as a few unit cells, generating highly concentrated scars. Depending on the symmetry of the confinement, the scars can have different shapes. However, the line segments forming these scars can only assume three directions. Figure 23(c) shows two symmetrical closed orbits. One can see that the line patterns are indeed quite sharp.

Effects of lattice orientation. The $E-\boldsymbol{k}$ structure of graphene has a hexagonal symmetry for the locations of the Dirac points in the wavevector space [c.f., Fig. 23(d)], even though the two nearby Dirac points are not equivalent. Figures 24 and 25 show some typical scars for the same stadium confinement with different lattice orientations, e.g., zigzag and armchair in the horizontal direction, respectively. In the high-energy regime, the orientations of the scars are determined by trigonal warping that have only three directions pointing from the origin to the Dirac points. As the $E-\boldsymbol{k}$ structures in Figs. 24 and 25 have a $\pi / 2$ rotational difference, the patterns of the scars in the two figures also exhibit a $\pi / 2$ difference. This explains the horizontal line patterns in Fig. 24 and the vertical line patterns in Fig. 25 in the high energy regime. The final scar formation also depends on the confinement shape, so that its symmetry will be manifested in the scars. Due to the discrete nature of the lattice, in the high-energy regime where the quantum wavelength is on the 


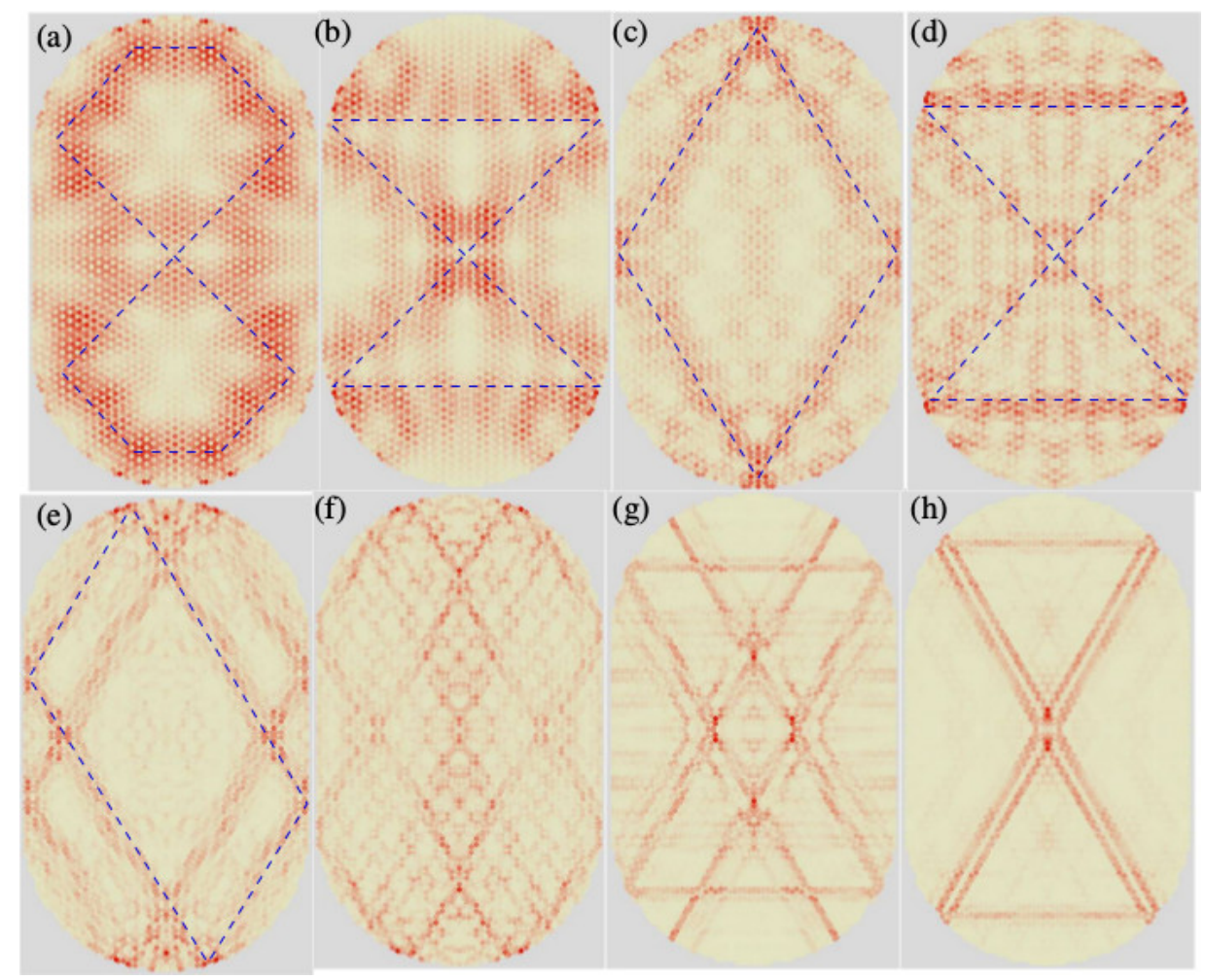

FIG. 24. Typical scars in the chaotic stadium graphene billiard with zigzag straight boundaries. A number of quantum scars for the same system as in Fig. 23. The energy values for (a-h) are $E / t=$ $0.25347,0.36358,0.57665,0.60699,0.81956,0.91061,0.97722$, and 0.99198 , respectively. The dashed lines represent the corresponding classical periodic orbits. From Ref. [121] with permission.

order of the lattice constant, the roughness at the edge needs to be taken into account to determine the effective shape of the billiard. Furthermore, only when a closed orbit has segments in the three allowed directions, as determined by the lattice orientation, can a scar be formed.

Recurrence of relativistic quantum scars. As the energy or the wave number varies, scars can appear and disappear. From the semiclassical theory [324], two successive scars associated with the same classical periodic orbit can occur when the action integral satisfies the condition $\Delta S=h$. Since $S=\oint \boldsymbol{p} \cdot \mathrm{d} \boldsymbol{q}$ and $\boldsymbol{p}$ has the same direction as d $\boldsymbol{q}$, for a given periodic orbit of length $L$, one has

$$
S=|\boldsymbol{p}| L=\hbar|\boldsymbol{k}| L .
$$

Thus the condition in terms of $\boldsymbol{k}$ is $\Delta|\boldsymbol{k}|=2 \pi / L$. For a conventional semiconductor confinement, the electron energy is $E=\hbar^{2} k^{2} /\left(2 m_{e}\right)$. In this case, $\sqrt{E}$ is equally spaced for scars associated with the same periodic orbit [325-327]. For a graphene confinement, one has $E=\hbar v_{F} k$ in the low energy regime, where $v_{F}=\sqrt{3} t a /(2 \hbar)$ is the Fermi velocity, $k=|\Delta \boldsymbol{k}|$ is the magnitude of the wavevector from the Dirac points, so it is the energy $E$ which is equally spaced for recurring scars. In particular, the energy interval for scars concentrating on a given periodic orbit is determined by $h=\hbar \Delta|\boldsymbol{k}| L=\left(\Delta E / v_{F}\right) L$, or $\Delta E=h v_{F} / L$. Series of recurring patterns were identified for the one shown in Fig. 23(b) and the one in Fig. 25(a), and their energies are plotted in Fig. 26. In Fig. 23(b), the energy interval between consecutive scars is $0.0195 t$ and the length of the orbit is $275 a=67.65 \mathrm{~nm}$, leading to $\Delta E=0.0198 t$. For scars similar to Fig. 25(a), the energy interval is 


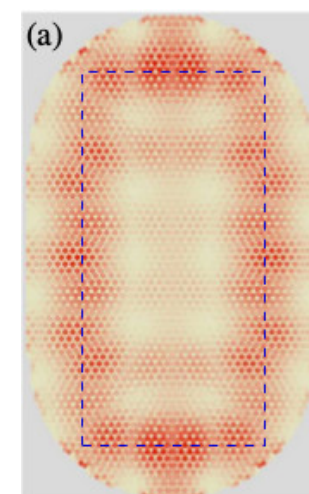

(e)

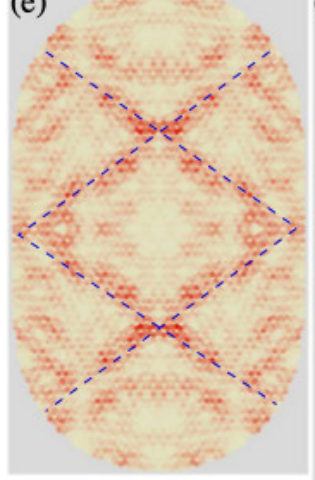

(b)

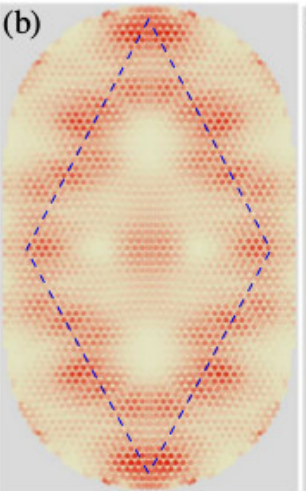

(f)

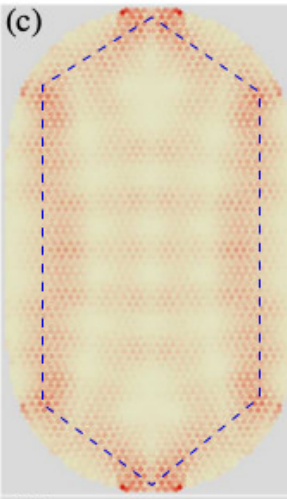

(g) (d)

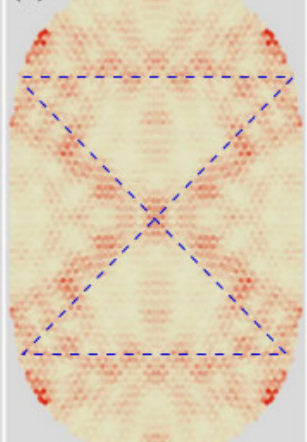

(h)

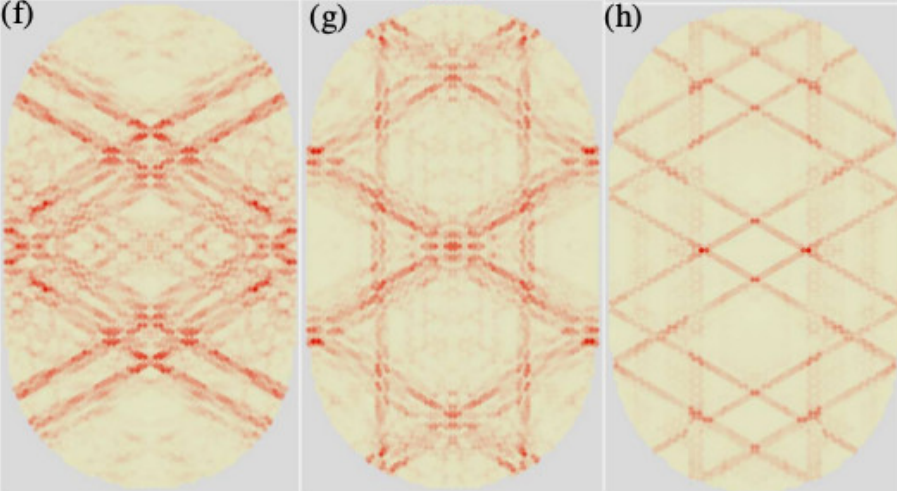

FIG. 25. Quantum scars in a chaotic stadium graphene billiard with armchair straight boundaries. The billiard has $N=13694$ carbon atoms. The corresponding energy values are $E / t=0.20031,0.2599$, $0.3106,0.54954,0.59238,0.9168,0.95216$, and 0.99801 for $(\mathrm{a}-\mathrm{h})$, respectively. From Ref. [121] with permission.

$0.0203 t$, while the length of the orbit is $263 a=64.70 \mathrm{~nm}$, yielding $\Delta E=0.0207 t$, which agrees well with the numeric observations [121]. This corroborates the linear relation between energy and wavevector, which is a unique feature of scarring in graphene systems and distinct from that in nonrelativistic quantum systems. Recently, this feature of equal spacing of $E$ in the recurring scarring states has been verified experimentally in a mesoscopic graphene ring system [328].

Scars in the stadium shaped graphene confinement in the presence of a perpendicular magnetic field were also investigated. As the band structure of graphene changes due to the magnetic field, the scars vary accordingly. It was found that, for a weak field, the corresponding classical orbits of the scars are almost unchanged, but when the field is strong, the scars are smeared out and some new orbits (both straight and curved) appear.

\section{Chiral scars in chaotic Dirac billiards}

Chiral scars in chaotic Dirac billiards were discovered [123] in 2013, aided by the conformalmapping method that enables a large number of eigenvalues and eigenstates to be calculated accurately and efficiently. In general, relativistic quantum scars, the eigenstates of the Dirac equation in a closed chaotic domain, can be classified into two categories, depending on whether the corresponding classical periodic orbits have an even or an odd number of bounces off the domain 


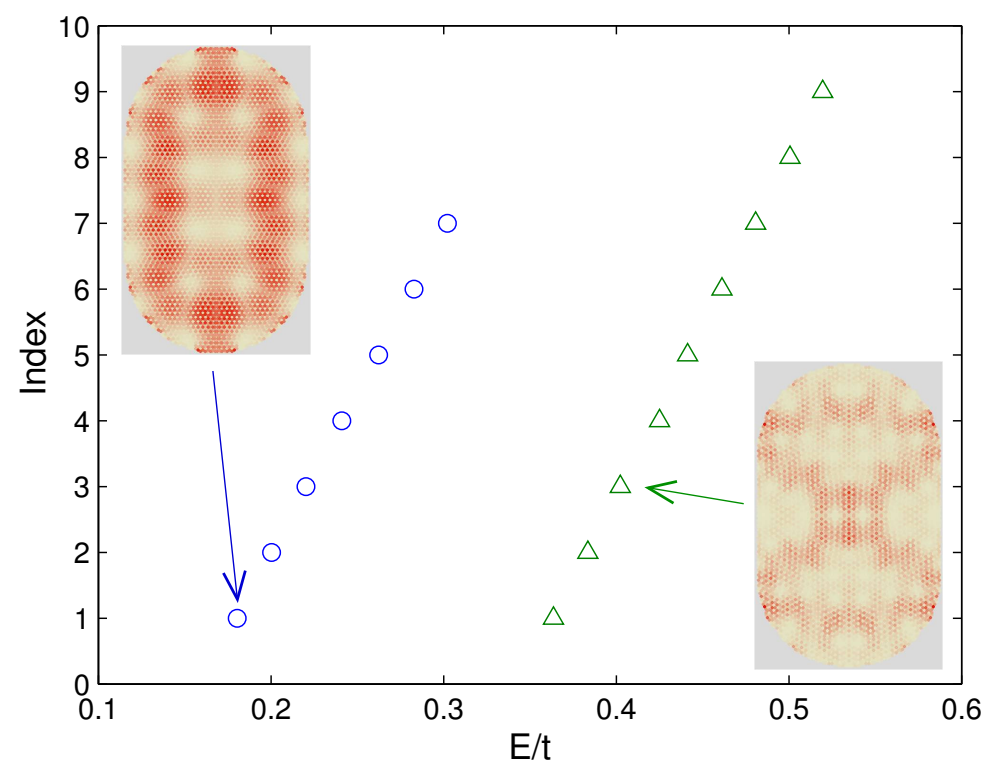

FIG. 26. Recurrence of quantum scars in the chaotic stadium graphene billiard. For the scar shown in Fig. 23(b) and the one in Fig. 25(a), the energy values at which they recur. The index represents relative values. From Ref. [121] with permission.

boundary in one circulation. In particular, those with an even number of bounces are "normal" scars as in nonrelativistic quantum systems in that the phase difference between the spinor after one itinerary about the orbit and its time reversed counterpart is an integer multiple of $2 \pi$, so they are not distinguishable. However, the scars associated with an odd number of bounces gain an extra phase difference of $\pi$ for counterclockwise and clockwise orbits after one circulation, so the scars with different orientations can be distinguished. These are chiral scars that absolutely have no counterparts in nonrelativistic quantum systems. Chiral scars are thus uniquely a relativistic quantum phenomenon with its origin in the chirality of the massless Dirac fermion.

Chiral scars were discovered through a class of chaotic Dirac billiards $\mathcal{B}$ whose boundaries are described by the following complex function $w(z)$ in Eq. (54). The quadratic conformal map has the advantage of amenability to analytic treatment where, in particular, the $\phi$ integral in Eq. (60) can be found explicitly and the matrix $M_{l m l^{\prime} m^{\prime}}$ becomes nearly diagonal in $l$. The conformal-mapping method can yield a large number of eigenenergy levels and the corresponding eigenstates, e.g., 15000 eigenvalues and eigenstates at unprecedentedly high accuracy when 40000 basis functions are employed [123].

Aided by numerical computations, the physical reasoning leading to the discovery of the chiral scars is as follows. An examination of a large number of scars for massless Dirac fermion in the chaotic billiard revealed that certain scarring patterns, once having appeared, tend to recur at a different energy value, as in nonrelativistic quantum systems. According to the semiclassical theory [152], two repetitive scars associated with the same classical periodic orbit can occur when the action difference satisfies the relation $|\Delta S|=2 \pi n \hbar(n=1,2, \cdots)$, where

$$
S=\oint \boldsymbol{p} \cdot \mathrm{d} \boldsymbol{q}=\hbar k L
$$

and $L$ is the length of the periodic orbit. If a scar has already appeared, say, at $k_{0}$, there is a high 

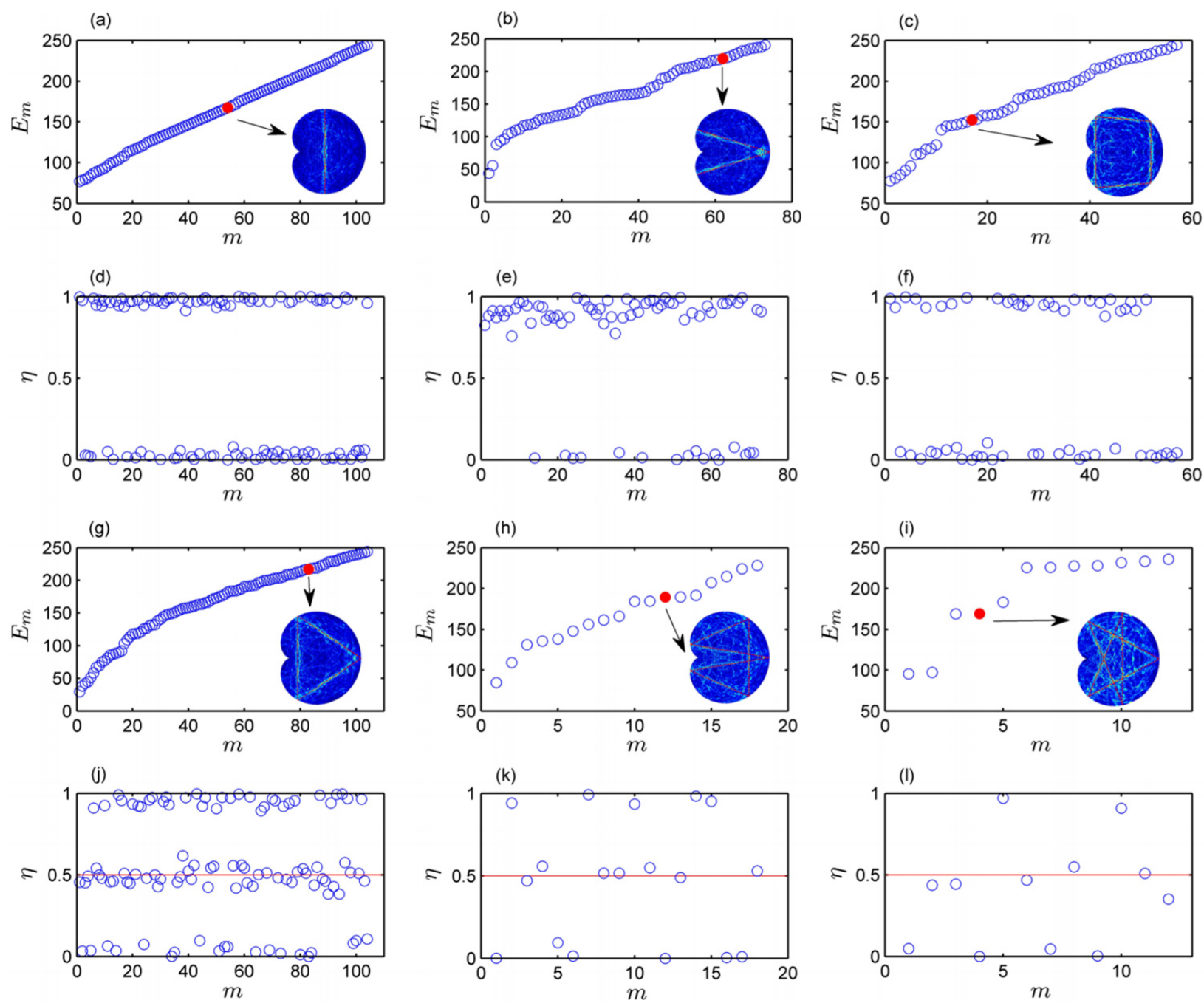

FIG. 27. Evidence of chiral scars for massless fermions in a chaotic Dirac billiard system. For a chaotic domain whose boundary is obtained through conformal mapping of the unit circle, (a-c) the energy levels $E_{m}$ versus the sequence number $m$ for the scar types 2, 4-I, and 4-II in Table III, respectively. (d-f) The semiclassical quantity $\eta$ [Eq. (120)] versus $m$ with the relevant data from panels (a-c), respectively. (g-l) Results for the scar types 3, 5-I, and 5-II. From Ref. [123] with permission.

likelihood that the eigenfunctions associated with the wave numbers $k_{n}=k_{0} \pm n \delta k$ will scar, where $\delta k=2 \pi / L$. One can then define the following characterizing quantity:

$$
\eta(n)=\frac{\left|k_{n}-k_{0}\right|}{\delta k}-\left[\frac{\left|k_{n}-k_{0}\right|}{\delta k}\right],
$$

where $[x]$ denotes the largest integer less than $x$. For a nonrelativistic quantum system, the semiclassical theory stipulates that $\eta$, by its definition, can exhibit only two distinct values: either approximately zero or about one. The calculation of the value of $\eta$ requires some parameters characterizing the underlying quantum scars. Table III lists some key features of the scars for the chaotic Dirac billiard system. Using the data of the typical types of scars, i.e., scar types 2, 3, 4 and 5 in Table III, one can calculate the values of $\eta(n)$ from Eq. (120), as shown in Fig. 27(a-1), For 

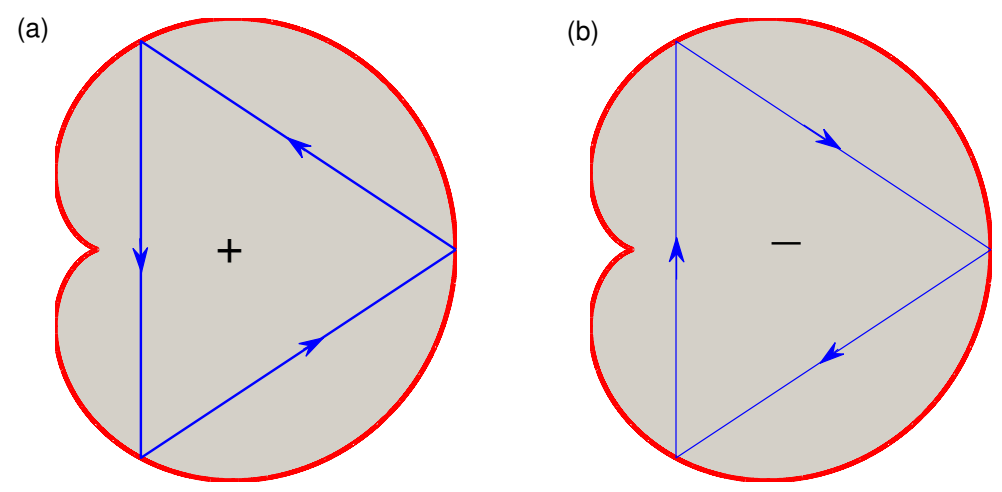

FIG. 28. Physical origin of chiral scar for massless fermions in a chaotic Dirac billiard. Illustration of a pair of orbits with opposite orientations.

scar types 2 and 4, $\eta(n)$ exhibits the two "normal" values, i.e., zero or one, as can be anticipated from the semiclassical theory. However, for scar types 3 and 5, $\eta$ can attain the additional value of $1 / 2$, violating the conventional semiclassical prediction.

TABLE III. Characteristics of relativistic quantum scars in a chaotic Dirac billiard.

\begin{tabular}{lcccc}
\hline \hline Scar index $^{\mathrm{a}}$ & $L$ & $\delta k$ & $k_{0}$ & Collected number \\
\hline 2 & 4.2425 & 1.4810 & 167.3225 & 104 \\
$4-\mathrm{I}$ & 7.5385 & 0.8335 & 219.8747 & 73 \\
$4-\mathrm{II}$ & 5.7993 & 1.0843 & 152.2197 & 57 \\
& & & & 104 \\
3 & 5.3764 & 1.1687 & 217.0473 & 18 \\
5 -I & 8.4725 & 0.7416 & 189.2712 & 12 \\
5 -II & 9.7321 & 0.6456 & 169.0422 & \\
\hline \hline
\end{tabular}

a The relativistic quantum scars are labeled as $n$ - the period of the corresponding classical periodic orbit, if no other configurations exist. For orbits of the same period but with a different configuration, Roman numerals are used.

The origin of the "abnormal" chiral scars that do not obey the conventional semiclassical quantization rules can be understood by considering the chirality of massless Dirac fermions and analyzing the associated phase changes. In particular, for a classical periodic orbit, roughly chirality can be understood as the cumulative effect of reflections at the billiard wall. For example, let us consider one pair of orbits that close on themselves after $N$ bounces but with opposite orientation, as shown schematically in Fig. 28. In terms of the plane waves, after traversing the orbit once, the accumulated phase change due to the spin rotation at reflections is $\Omega=\frac{1}{2}\left(\theta_{N}-\theta_{0}\right)$, which can be obtained by the reflection law $\theta_{n+1}=\pi+2 \widetilde{\theta}(s)-\theta_{n}$ for $n=0,1, \cdots, N$, where $\widetilde{\theta}(s)$ is the angle between the normal vector of the boundary at the reflection point and the positive $x$-axis. One can then define $\Omega_{+}-\Omega_{-}$as the phase difference between a pair of counterclockwise $(+)$ and clockwise (-) orbits. Berry and Mondragon obtained [93] the following relations: $\Omega_{+}-\Omega_{-}$ is equal to integer multiples of $2 \pi$ for even $N$, but there will be an extra $\pi$ in $\Omega_{+}-\Omega_{-}$for odd 
$N$. Since chirality corresponds to the situation of $\Omega_{+}-\Omega_{-}$being $\pi$ (modulus $2 \pi$ ), where the two orbits in the pair enclose themselves with an opposite sign change, the scars with an even number of bounces are not chiral but those with an odd number of bounces are. Chirality can thus have a remarkable effect on scarring, leading to the value of $1 / 2$ for $\eta$, which absolutely cannot arise in nonrelativistic quantum systems.

\section{Fundamental mechanism of chiral scars and their control via an external magnetic field}

\section{Symmetry considerations}

Time-reversal $(\mathcal{T}$-)symmetry, a fundamental symmetry in physical systems [154, 329-331], plays a key role in chiral scars. In general, magnetism can be used to break the $\mathcal{T}$-symmetry for a microscopic process. For example, the classical scenario for a system to break the $\mathcal{T}$-symmetry is a charged particle moving in a magnetic field, whose time-reversed orbit is no longer a solution of the system [295, 332]. In quantum physics, $\mathcal{T}$-symmetry breaking can be more subtle in that the time-reversed state can be the same but with a different phase in the action integral, leading to remarkable physical phenomena such as the Aharonov-Bohm (AB) effect [256, 333]. Similarly, a ferromagnetic perturbation in the electromagnetic wave analog of the Schrödinger equation breaks the $\mathcal{T}$-symmetry in microwave billiards [258, 334].

Berry and Mondragon discussed the subtle $\mathcal{T}$-symmetry breaking phenomenon for 2D massless Dirac particles confined in a finite region [93]. In this case, the $\mathcal{T}$-symmetry is broken naturally even without any magnetic field. The resulting level-spacing statistics of the chaotic Dirac billiard exhibit features typical of the Gaussian unitary ensemble (GUE) statistics (Sec. III). Graphene, however, in general does preserve the $\mathcal{T}$-symmetry, due to the existence of two non-equivalent Dirac points. In particular, extensive searches of $\mathcal{T}$-breaking in graphene billiards were conducted $[115,118,119,282,299,316,335]$ with the finding that, only under special circumstances, violation of the valley symmetry that leads to breaking of the $\mathcal{T}$-symmetry and consequently to GUE level spacing statistics, can occur $[119,316]$.

Mathematically, $\mathcal{T}$-symmetry breaking of the confined Dirac particle arises because the confinement potential is a scalar four potential [93], so that the Hamiltonian does not commute with the time reversal operator. Consequently, the operator generating the boundary condition imposed by the confinement potential does not commute with the time reversal operator either. Berry and Mondragon provided a semiclassical understanding through analyzing the phase difference between the plane waves traveling in one direction of the periodic orbit and its time-reversed counterpart [93]. They found that, for orbits with an even number of bounces off the domain boundary, the accumulated phase difference between the clockwise and counterclockwise orbits is an integer multiple of $2 \pi$, which does not break the time reversal symmetry. However, the orbits with an odd number of bounces have an additional $\pi$ in the accumulated phase difference, making distinct the counterclockwise and the clockwise motions. As a result, for those orbits, the $\mathcal{T}$-symmetry is broken. The chiral property of scars is thus closely related to the overall difference in the phase change.

The $\mathcal{T}$-breaking mechanism in Dirac billiard systems had not been fully understood, especially from the semiclassical perspective. An alternative approach was to consider the reflections of the planar Dirac spinor wave at the boundary interface of a straight potential jump. It was noted [137] that there can be a non-vanishing probability current density along the boundary even when the 
scalar four-potential energy tends to infinity. Furthermore, the current flow is orientated (i.e., fixed with respect to a reference direction), which is independent of the incident angle, even though the magnitude of the current will be affected. Thus the time-reversed orbit of the planar spinor wave will result in an asymmetric current at the boundary, which breaks the $\mathcal{T}$-symmetry in accordance with the non-commutable relation between the $\mathcal{T}$-operator and the boundary condition [93].

Since the spin operator is proportional to the current operator, spin polarization at the boundary is also fixed and independent of the incident angle, e.g., whether upward or downward. The wave at the boundary is in fact an eigenfunction of the spin operator $\hat{S}_{y}$ with the eigenvalue of $\hbar / 2$, regardless of the incident angle. That is, regardless of whether the incident wave is coming upward or downward, the spin always points up (or counterclockwise), indicating chirality. As a result, a time-reversed wave will not result in a time-reversed spin polarization at the boundary, leading to $\mathcal{T}$-breaking.

\section{Time-reversal symmetry breaking and chiral scars}

To understand the physical origin of chiral scars and the role played by $\mathcal{T}$-symmetry breaking, the idea of studying chaotic Dirac billiard systems in the presence of an external magnetic field, i.e., AB billiard, was articulated [124]. A magnetic flux can induce phase changes for the spinor [93] which, under the right conditions, can compensate the subtle $\pi$ phase change associated with chiral scars. It is also possible to "control" the chirality in the sense that, by tuning the magnetic flux, a chiral scar can become non-chiral, and vice versa. In fact, an exact semiclassical formula can be worked out for the phase accumulation [124].

A chaotic Dirac AB billiard with a vanishing inner radius [137] represents a relatively simple setting that retains the essential physics pertinent to chiral scars [124]. Because of the vanishing region in which a magnetic field is present, the classical orbit for a chiral scar is not disturbed but the magnetic flux can still induce an additional phase to the quantum spinor. In an experimental setup, the inner radius is necessarily finite. However, insofar as the region through which the magnetic flux passes does not intersect with the scar orbit, the effect of the field on the scarred orbit is negligible.

The origin of the $\pi$ phase change associated with chiral scars can be attributed to the boundaryspin interaction of the spinor plane waves. At each collision, the phase difference between the counterclockwise reflection and its time reversed counterpart has an additional $\pi$ contribution, leading to spin polarization at the boundary. As a result, for a scar on an orbit with an even number of reflections, the spin-boundary interaction contributes an integer multiple of $2 \pi$. In this case, the $\mathcal{T}$-symmetry is preserved for the complete orbit. However, for the scars with an odd number of reflections, the boundary phases contribute an additional $\pi$ (modulus $2 \pi$ ), leading to $\mathcal{T}$-symmetry breaking.

Current analysis of scars. It is convenient to study the local current flows to investigate the phase of the scarred eigenstates. The current operator is [93]

$$
\hat{j}=\nabla_{p} \hat{H}=c \hat{\boldsymbol{\sigma}}
$$

and the local current for state $\Psi$ can be defined as the expectation value of $\hat{j}$ :

$$
\boldsymbol{j} \equiv c\left(\psi_{1}^{*}, \psi_{2}^{*}\right) \hat{\boldsymbol{\sigma}}\left(\begin{array}{c}
\psi_{1} \\
\psi_{2}
\end{array}\right)=2 c\left[\operatorname{Re}\left(\psi_{1}^{*} \psi_{2}\right), \operatorname{Im}\left(\psi_{1}^{*} \psi_{2}\right)\right]
$$



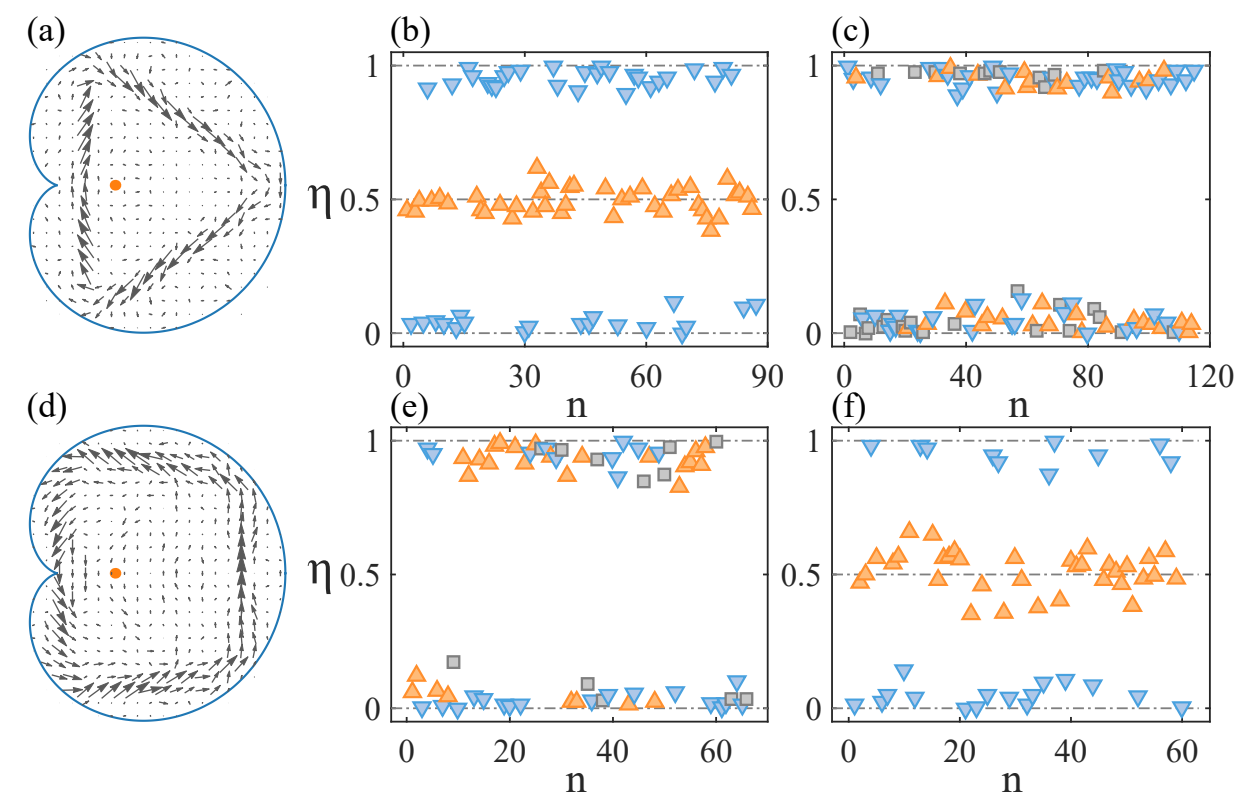

FIG. 29. Directions of currents associated with scars in an AB chaotic Dirac billiard. Scarring currents (a,d) and the corresponding $\eta$ values for $\alpha=0(\mathrm{~b}, \mathrm{e})$ and $\alpha=1 / 4(\mathrm{c}, \mathrm{f})$. The first row is for a period-3 scar, and the second row is for a period-4-II scar. The orange up-triangles and blue down-triangles are for scars with a counterclockwise and a clockwise flow, respectively, and the gray squares are for scars whose current orientation cannot be distinguished. The reference state is chosen (arbitrarily) from the scars with a clockwise flow. A singular magnetic flux passes through the central region of the domain that does not intersect with the scarred orbits. From Ref. [124] with permission.

A systematic investigation [124] of the local current flow for scarred states indicated that the currents have a definitive orientation, either clockwise or counterclockwise, as shown in Fig. 29(a) and 29(d) for a period-3 and a period-4 orbit, respectively. The relation between $\eta$ and the scar orientation defined by its current flow can also be analyzed. For example, in Fig. 29, the scarred states with counterclockwise and clockwise flows are marked as orange up triangles and blue down triangles, respectively. For scars with an even number of bounces, the value of $\eta$ is zero or one, regardless of relative current orientation, as shown in Fig. 29(e). For an orbit with an odd number of bounces, when the scarred state and the reference scar have the same current orientation, one has $\eta=0$ or 1 . However, if the two scarred states have opposite current orientations, one has $\eta=1 / 2$, as shown in Fig. 29(b), indicating $\mathcal{T}$-symmetry breaking from the semiclassical point of view. This current orientation analysis confirms that $\eta=1 / 2$ is a result of the $\pi$ phase difference of the opposite current orientation of scars with an odd number of bounces.

Physics of local reflection. The simple model of plane wave reflecting from a straight potential $[93,137]$, as shown in Fig. 30, can be used to understand the reflection of a Dirac spinor at a boundary point. The fact that there is a $\pi$ phase difference between the phase change at reflection and its time reversed counterpart can be exploited to understand the difference in the global phase changes associated with orbits with an even and an odd number of bounces [93]. The analytic results are listed below [Eqs. (123-129)]. The wave (incident plus reflected) in the free region 
(a)

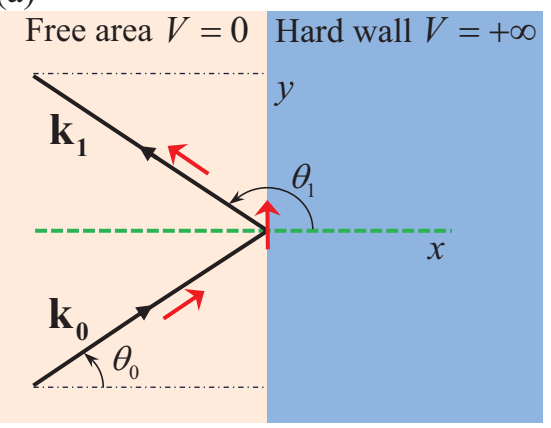

(b)

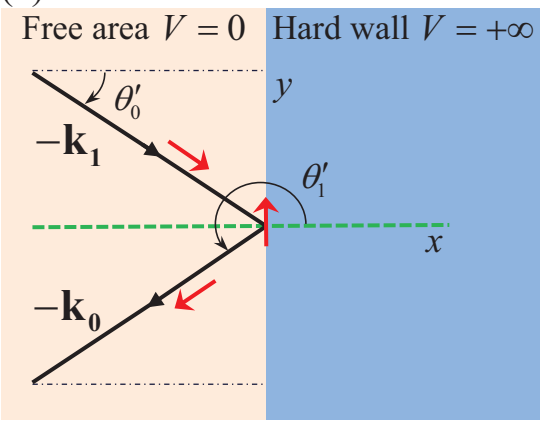

FIG. 30. Setting of a simple spinor reflection/transmission model. (a) A local reflection and (b) its time reversed counterpart. Shown are incident and reflected plane waves (black), and the spin (vertical red arrow at the boundary) corresponding to the superposition of waves. From Ref. [124] with permission.

(region $\mathrm{I}, V=0$ ) can be written as

$$
\Psi^{\mathrm{I}}=\frac{1}{\sqrt{2}}\left[\left(\begin{array}{c}
\exp \left\{-\frac{1}{2} i \theta_{0}\right\} \\
\exp \left\{\frac{1}{2} i \theta_{0}\right\}
\end{array}\right) \exp \left\{i \boldsymbol{k}_{0} \cdot \boldsymbol{r}\right\}+R\left(\begin{array}{c}
\exp \left\{-\frac{1}{2} i \theta_{1}\right\} \\
\exp \left\{\frac{1}{2} i \theta_{1}\right\}
\end{array}\right) \exp \left\{i \boldsymbol{k}_{1} \cdot \boldsymbol{r}\right\}\right],
$$

and the transmitted wave in the potential region (region II) is

$$
\Psi^{\mathrm{II}}=\frac{T}{\sqrt{2}}\left(\begin{array}{c}
-i \chi_{1} \\
\chi_{2}
\end{array}\right) e^{-q x} e^{i K y}
$$

where the origin is at the boundary, $R, T$ are the reflection and transmission coefficients, respectively, the incident and reflected wave vectors are

$$
\begin{aligned}
& \boldsymbol{k}_{0}=\left(k \cos \theta_{0}, k \sin \theta_{0}\right) \text { and } \\
& \boldsymbol{k}_{1}=\left(k \cos \theta_{1}, k \sin \theta_{1}\right),
\end{aligned}
$$

respectively. Other quantities are

$$
\begin{aligned}
K & =k \sin \theta_{0}, \\
q & =\sqrt{\frac{V^{2}-E^{2}}{\hbar^{2} c^{2}}+K^{2},} \\
E & =\hbar c k, \\
\chi_{1} & =\sqrt{\frac{(V+E)(q-K)}{V q-E K}}, \text { and } \\
\chi_{2} & =\sqrt{\frac{(V-E)(q+K)}{V q-E K}} .
\end{aligned}
$$

Matching the two waves at $x=0$ and using the convention of specularity to relate the incident and reflected directions [93] yield

$$
\theta_{1}=\pi+2 \widetilde{\theta}(s)-\theta_{0}
$$


where $\widetilde{\theta}(s)$ is the angle between the normal vector of the boundary and the positive $x$-axis, which is zero for the case treated here. One obtains

$$
R=\frac{i \lambda-e^{i \theta_{0}}}{i-\lambda e^{i \theta_{0}}}=e^{i\left(2 \gamma+\theta_{0}-\frac{\pi}{2}\right)}
$$

where the parameters $\gamma$ and $\lambda$ are defined through

$$
\begin{gathered}
\tan \gamma=\frac{\chi_{1}-\chi_{2} \sin \theta_{0}}{\chi_{2} \cos \theta_{0}}=\frac{1-\lambda \sin \theta_{0}}{\lambda \cos \theta_{0}} \\
\lambda=\frac{\chi_{2}}{\chi_{1}}=\sqrt{\frac{(V-E)(q+K)}{(V+E)(q-K)}}=\frac{V-E}{\hbar c(q-K)}=\frac{\hbar c(q+K)}{V+E} .
\end{gathered}
$$

The transmission coefficient is given by

$$
T=\frac{2 \cos \gamma}{\chi_{2}} e^{i\left(\gamma+\frac{\theta_{0}}{2}\right)} .
$$

The convention in Eq. (125) implies the change from $\theta_{0}$ to $\theta_{1}$ when the angle is rotated counterclockwisely, as shown in Fig. 30. If the change in the angle is due to the clockwise rotation, there will be an additional $2 \pi$ at the right side of Eq. (125), and an additional phase $\pi$ in the plane wave in the second term of Eq. (123) because of the prefactor $1 / 2$. However, the end results are unchanged. Another feature of the refection coefficient $R$ is $R\left(\theta_{0}\right)=R\left(-\theta_{0}\right)$, i.e., the coefficient has the same value for forward or backward incidence. For a finite value of $V$ and $V>E, R$ is not a constant but depends on the position and angle of incidence. As the value of $V$ becomes large, the value of $R$ tends to one. Analysis can be simplified (but without loss of essential physics) by assuming $E>0, V \rightarrow \infty$, and $R=1$.

Spin orientation. The spinor wavefunction at the boundary in Fig. 30(a) is

$$
\Psi^{\mathrm{I}}=\left(\begin{array}{c}
\exp \left\{-\frac{1}{2} i \theta_{0}\right\}+\exp \left\{-\frac{1}{2} i \theta_{1}\right\} \\
\exp \left\{\frac{1}{2} i \theta_{0}\right\}+\exp \left\{\frac{1}{2} i \theta_{1}\right\}
\end{array}\right)=\left(\begin{array}{c}
\exp \left\{-\frac{1}{2} i \theta_{0}\right\}-i \exp \left\{\frac{1}{2} i \theta_{0}\right\} \\
\exp \left\{\frac{1}{2} i \theta_{0}\right\}+i \exp \left\{-\frac{1}{2} i \theta_{0}\right\}
\end{array}\right) .
$$

In Fig. 30(b), the wavefunction is

$$
\Psi^{\mathrm{II}}=\left(\begin{array}{c}
\exp \left\{-\frac{1}{2} i \theta_{0}^{\prime}\right\}+\exp \left\{-\frac{1}{2} i \theta_{1}^{\prime}\right\} \\
\exp \left\{\frac{1}{2} i \theta_{0}^{\prime}\right\}+\exp \left\{\frac{1}{2} i \theta_{1}^{\prime}\right\}
\end{array}\right)=\left(\begin{array}{c}
\exp \left\{-\frac{1}{2} i \theta_{0}^{\prime}\right\}-i \exp \left\{\frac{1}{2} i \theta_{0}^{\prime}\right\} \\
\exp \left\{\frac{1}{2} i \theta_{0}^{\prime}\right\}+i \exp \left\{-\frac{1}{2} i \theta_{0}^{\prime}\right\}
\end{array}\right) .
$$

The spin operator in the $y$-direction is $\hat{S}_{y}=(\hbar / 2) \hat{\sigma}_{y}$. Both $\Psi^{\mathrm{I}}$ and $\Psi^{\mathrm{II}}$ are eigenfunctions of $\hat{S}_{y}$ and share the same eigenvalue $\hbar / 2$ :

$$
\hat{S}_{y} \Psi^{\mathrm{I}, \mathrm{II}}=\frac{\hbar}{2} \Psi^{\mathrm{I}, \mathrm{II}}
$$

Physically, this means that the two opposite incident directions have the same spin orientation at the boundary - a remarkable feature of the Dirac spinor! The spin thus points to the counterclockwise direction regardless of the incident angle, which is stipulated by the boundary condition as well. This indicates that, at each local collision, the $\mathcal{T}$-symmetry is broken due to the interaction between spin and the infinite mass boundary. (Note that, while in the configuration space the probability density currents on the boundary have the same orientation for the two opposite incident directions, the magnitudes are typically different [137].)

Additional $\pi$ phase for reversed reflection. It is now feasible to analyze the phase change of the spinor wavefunction during one reflection for $V \rightarrow+\infty$ and $R=1$. Let the incident and reflected 
angles be $\theta_{0}$ and $\theta_{1}$, respectively, as shown in Fig. 30(a). The two angles are related by Eq. (125). From Eq. (123), the phase difference between the two directions becomes

$$
\delta_{+}=\frac{1}{2}\left(\theta_{1}-\theta_{0}\right)=\frac{1}{2}\left[\pi+2 \widetilde{\theta}(s)-2 \theta_{0}\right],
$$

For the reversed reflection direction, the incident and reflected angles are labeled as $\theta_{0}^{\prime}$ and $\theta_{1}^{\prime}$, where $\theta_{0}^{\prime}=-\theta_{0}+2 \widetilde{\theta}(s)+2 n \pi$ and $n$ is an integer, as shown in Fig. 30(b). The phase difference between the two directions is

$$
\delta_{-}=\frac{1}{2}\left(\theta_{1}^{\prime}-\theta_{0}^{\prime}\right)=\frac{1}{2}\left[\pi+2 \widetilde{\theta}(s)-2 \theta_{0}^{\prime}\right]=-\delta_{+}+\pi-2 n \pi,
$$

Note that $\tilde{\theta}(s)=0$ and the additional $2 n \pi$ factor has no observable effect, which can then be ignored. Each collision comes with a minus sign in the phase change of a pair of two opposite incident directions and there is an additional phase $\pi$. The minus sign is important that the difference in the phase change for each collision becomes

$$
\delta_{+}-\delta_{-}=2 \delta_{+}-\pi+2 n \pi
$$

Global phase change. The global phase changes can be obtained through the local phase change gained at each reflection. For a closed orbit with the initial incident angle $\theta_{0}$ from Eq. (131), the closure condition means $\theta_{N}-\theta_{0}=2 m \pi$, where $m$ is an integer that can be determined from Eq. (131). The whole phase change is

$$
\Delta_{+}=\frac{1}{2}\left(\theta_{N}-\theta_{0}\right)=m \pi
$$

If the initial direction of the same orbit is reversed, according to Eq. (132), the global phase change is given by

$$
\Delta_{-}=\frac{1}{2}\left(\theta_{N}^{\prime}-\theta_{0}^{\prime}\right)=-\Delta_{+}+N \pi
$$

where $N$ is the total number of reflections along the orbit. The total phase difference between the two reversed orbits due to the boundary reflections is then

$$
\Delta_{+}-\Delta_{-}=2 m \pi-N \pi
$$

which agrees with Eq. (133). For an odd number of bounces $N$, there will be a $\pi$ difference in the phase between the reversed orbits caused by boundary, while for an even number of bounces, there is no nontrivial phase difference. This is in agreement with the analysis of Berry and Mondragon [93].

\section{Change in scar chirality due to a magnetic flux}

The nontrivial phase change acquired through the boundary reflections can be compensated for by a magnetic flux [124]. In particular, let the magnetic flux be $\alpha$ (in units of magnetic flux quanta $\left.\Phi_{0} \equiv h / e\right)$ and the winding number be $W$ for a certain orbit around this flux. The phase gain due to the magnetic flux is $2 \pi W \alpha$. For a time reversed orbit, $W$ changes sign, so the phase difference between these two orbits with opposite orientations becomes $4 \pi W \alpha$. For $W=1$, if $\alpha=1 / 4$, the 


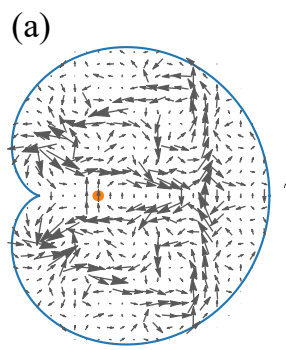

(d)

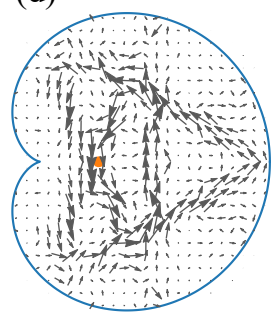

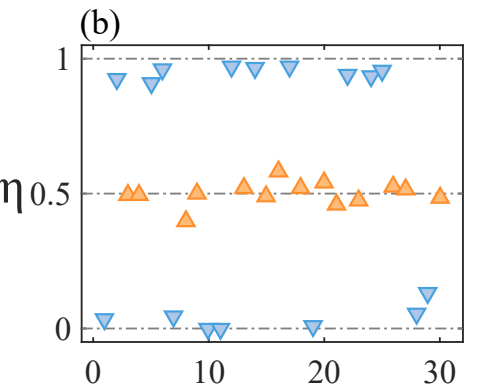

(e)

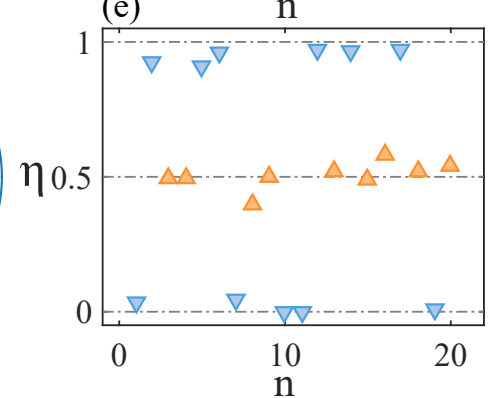

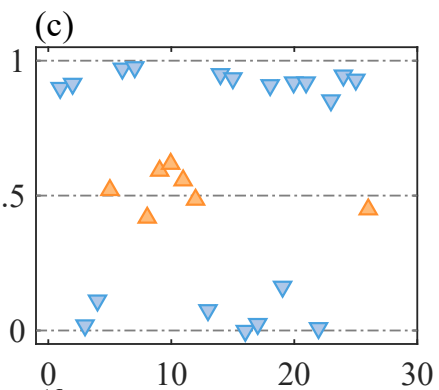

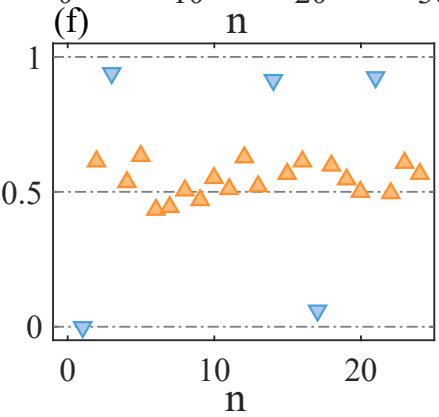

FIG. 31. Directions of currents associated with period-5 scars in the AB chaotic Dirac billiard. The same plots as in Fig. 29 except that the first and second rows are for the period-5-I and period-5-II scars listed in Tab. III, respectively. From Ref. [124] with permission.

magnetic flux will introduce a $\pi$ phase difference. This phase will be added to the phase acquired through boundary-spin interaction. As a result, for an odd orbit scar, the total phase difference becomes 0 modulus $2 \pi$, and they will lose the chiral character. For even orbit scars, the total phase will be $\pi$ (modulus $2 \pi$ ), and they will become chiral. As shown in Fig. 29, when there is no magnetic flux, i.e., $\alpha=0, \eta$ attains the value of 0.5 for the period-3 chiral scar. However, for $\alpha=1 / 4, \eta$ no longer possesses any value near 0.5 , indicating preservation of the time reversal symmetry. In contrast, for the period- 4 scar, the value of $\eta$ is zero or one for $\alpha=0$, but the value 0.5 emerges for $\alpha=1 / 4$, further indicating the equivalence between the boundary-spin interaction induced phase and that due to a magnetic flux.

Note that, for non-chiral scars, the two flow orientations are mixed, i.e., they can all take on values of zero or one for $\eta$. While for scars with a chiral nature, e.g., the period-3 scars at $\alpha=0$ and the period- 4 scars for $\alpha=1 / 4$, the scars with different orientations can be distinguished. For one set of the scars, $\eta$ can have the value of 0.5 , while for the other set, the value of $\eta$ is either zero or one.

Figure 31 presents the same results as in Fig. 29 but for two period-5 scars. Surprisingly, the value of $\eta$ for $\alpha=0$ and $\alpha=1 / 4$ appear the same. A more detailed examination [124] revealed that, for the period-5-I orbit, the flux has no effect because it is outside of and not circulated by the orbit, thus $W=0$. However, the period-5-II orbit circulates the flux twice, i.e., $W=2$. As a result, for $\alpha=1 / 4$, the phase difference between the counterclockwise and clockwise orbits is $4 \pi W \alpha=2 \pi$. This does not change the chirality of the scar. 


\section{Semiclassical formula of chiral scars}

Phenomenologically, as the phase caused by the boundary-spin interaction is equivalent to that due to a magnetic flux, it can be included in the semiclassical formulas for the phase shift $[2,326$, 327, 336]:

$$
\Delta \Phi=\frac{1}{\hbar} S-\frac{\sigma \pi}{2}+2 \pi \beta
$$

where the action is [256]

$$
S=\oint \boldsymbol{p} \cdot \mathrm{d} \boldsymbol{q}=\hbar \oint \boldsymbol{k} \cdot \mathrm{d} \boldsymbol{q}+e \oint \boldsymbol{A} \cdot \mathrm{d} \boldsymbol{q}=k \cdot L+2 \pi W \alpha .
$$

One thus has

$$
\Delta \Phi=k \cdot L+2 \pi W \alpha-\frac{\sigma \pi}{2}+2 \pi \beta,
$$

where $\sigma$ is the Maslov index that is the number of conjugate points along the orbit and is canonically invariant [337]. For the heart-shaped chaotic billiard, the value of $\sigma$ is nothing but the number of reflections along a complete orbit [338]. The infinite mass (or hard wall) reflection only contributes a phase in the spin term, thus has no contribution to the Maslov index. The quantity $2 \pi \beta$ is the phase accumulation of spin reflections at the boundary, whose value depends on the particular orbit and current orientation [Eq. (134)]. Note that, because of the chiral effect caused by the spin-boundary interaction, there is a $\pi$ difference in the term $2 \pi \beta$ between the reversed odd orbits. For semiclassically allowed orbits, the phase accumulation around one cycle should be an integer multiple of $2 \pi$, i.e., $\Delta \Phi=2 \pi n(n=1,2, \cdots)$ so as to ensure that the wavefunction is single-valued. One thus has

$$
k=\frac{2 \pi}{L}\left(n-W \alpha+\frac{\sigma}{4}-\beta\right) .
$$

For zero magnetic flux $(\alpha=0)$, one can define [124] $\Gamma=\bmod (k L / 2 \pi, 1)=\bmod (\sigma / 4-\beta, 1)$, which relates the semiclassical Maslov index $\sigma$ and the quantity $\beta$ from relativistic quantum mechanics. The values of the parameters $\sigma, \beta$ and $\Gamma[\operatorname{via} \bmod (\sigma / 4-\beta, 1)]$ are listed in Table IV for different orbits. Alternatively, the values of $\Gamma$ can be obtained numerically through $\bmod [k L /(2 \pi), 1]$ from the eigen wavevectors of the corresponding scars. The results are shown in Fig. 32. The numerical values of $\Gamma$ agree well with those from the semiclassical theory.

\section{Control of chirality of scars via an external magnetic field}

The analysis of the physical mechanism of chiral scars in the AB chaotic Dirac billiard suggests that the chirality of a scar can be controlled by tuning the external magnetic flux. The starting point of the analysis is the difference in the wavevectors of reversed scars of the same type:

$$
\Delta k= \begin{cases}2 \pi(\Delta n-2 W \alpha) / L & \text { even bounces }, \\ 2 \pi(\Delta n-2 W \alpha+\Delta \beta) / L & \text { odd bounces. }\end{cases}
$$

where $n$ is an integer and $\Delta \beta=1 / 2$ for orbits with an odd number of bounces. Whenever the condition $|2 W \alpha|=1 / 2$ is met for an orbit, the corresponding scars will switch from being chiral to non-chiral, as demonstrated in Fig. 29. 
(a)

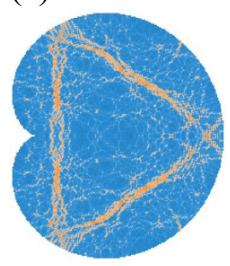

(e)

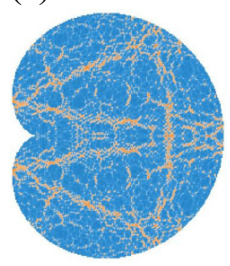

(i)

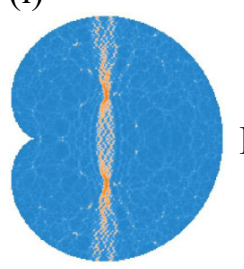

(b)
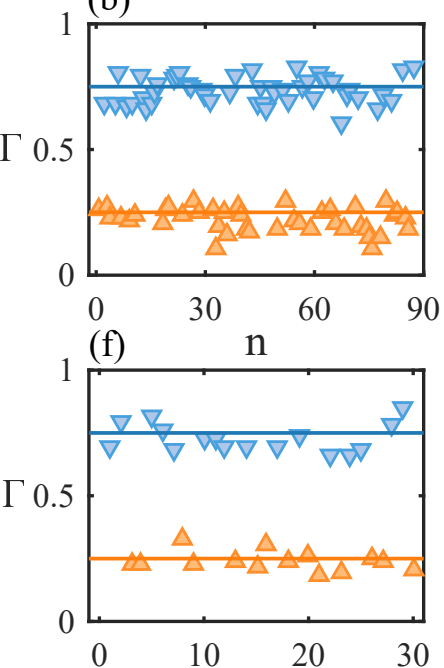

(j)

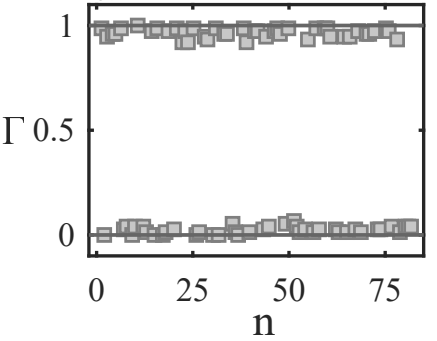

(c)

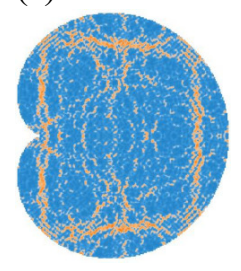

(g)

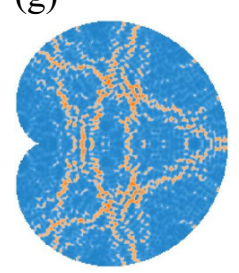

(k)

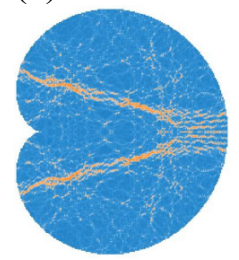

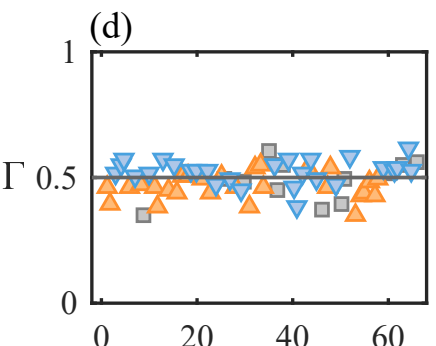

(h)

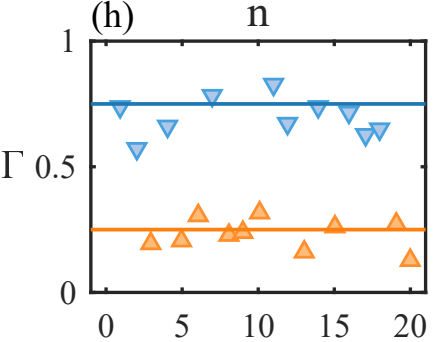

(1)

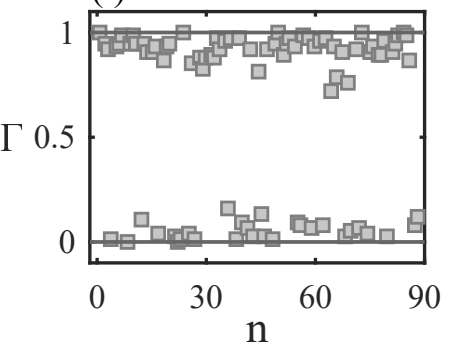

FIG. 32. Values of $\Gamma$ for different scars. The values of $\Gamma$ for scars of (a) period-3, (c) period-4-II, (e) period-5-I, (g) period-5-II, (i) period-2, and (k) period-4-I. Orange up-triangles and blue down-triangles represent scars with counterclockwise and clockwise current orientations, respectively. Gray squares correspond to scars without any apparent current orientation. The horizontal solid lines represent the semiclassical predictions from Table IV. From Ref. [124] with permission.
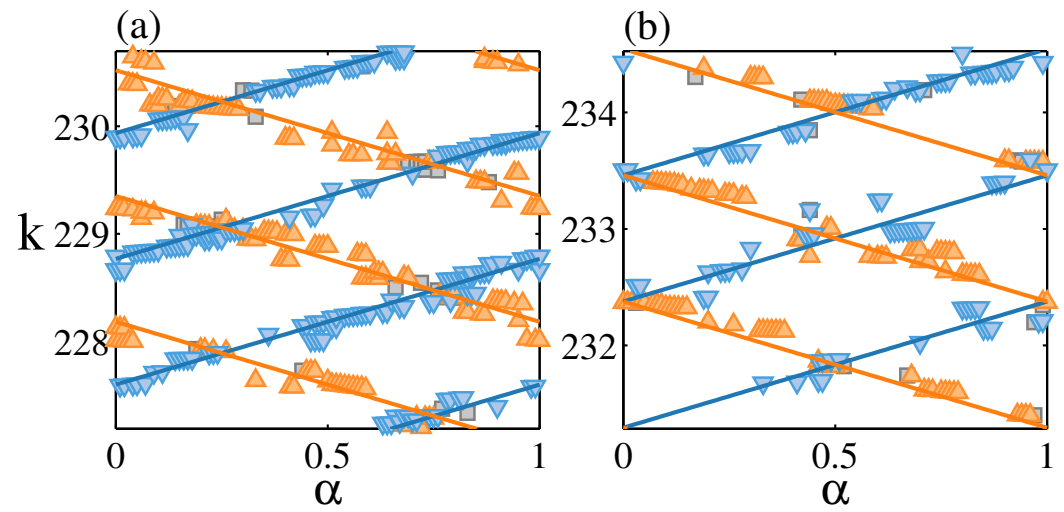

FIG. 33. Control of chirality of scars through an external magnetic flux. Shown are the relations between wavevector $k$ and magnetic flux $\alpha$, for (a) the period-3 scar in Fig. 29(a), and (b) the period-4-II scar in Fig. 29(d). The orange up-triangles indicate scars with a counterclockwise flow $(W=1)$, and the blue down-triangles are those with a clockwise flow $(W=-1)$. The gray squares mark the scars whose flow orientations cannot be identified. The solid lines are theoretical predictions of Eq. (140). The step in the variation of $\alpha$ is 0.01 . From Ref. [124] with permission. 
TABLE IV. Representative values of parameters in the semiclassical analysis of chiral scars. The values of $\sigma, \beta$ and $\Gamma$ are for different orbits (Fig. 32), where $(+,-)$ denote the counterclockwise and clockwise orientation, respectively.

\begin{tabular}{ccccccc}
\hline \hline Orbits & 2 & 3 & $4-\mathrm{I}$ & $4-\mathrm{II}$ & $5-\mathrm{I}$ & 5 -II \\
\hline$\sigma$ & 2 & 3 & 4 & 4 & 5 & 5 \\
$\beta$ & $1 / 2$ & $1 / 2(+)$ & 1 & $1 / 2$ & $1(+)$ & $1(+)$ \\
& & $0(-)$ & & & $1 / 2(-)$ & $1 / 2(-)$ \\
$\Gamma$ & 0,1 & $1 / 4(+)$ & 0,1 & $1 / 2(+)$ & $1 / 4(+)$ & $1 / 4(+)$ \\
& & $3 / 4(-)$ & & $1 / 2(-)$ & $3 / 4(-)$ & $3 / 4(-)$ \\
\hline \hline
\end{tabular}

According to Eq. (138), for a scar with wavevector $k_{0}$ for $\alpha=0$, as the magnetic flux $\alpha$ is increased, the same scar will appear for the wavevector value

$$
k=k_{0}-W \alpha \frac{2 \pi}{L}
$$

as $\beta$ depends only on the orbit and is fixed to a particular value for a given orbit. The scarring pattern is periodic as the magnetic flux varies from zero to one. In particular, as the magnetic flux is systematically varied, one can identify the scar on the same orbit in a certain wavevector (energy) range and specify their flow orientation [124]. Figure 33 shows the corresponding wavevector and magnetic flux for the period-3 and period-4-II scars [in Figs. 29(a) and 29(d), respectively]. The solid lines are from Eq. (140), which holds for periodic orbits with either an odd or an even number of bounces. The difference, however, comes from the initial $k_{0}$ value. From Fig. 33, it can be seen that, for the scars on any orbit, there are actually two sets: one with counterclockwise flow ( $W=1$ ) for which $k$ decreases linearly with increasing $\alpha$, and another with clockwise flow ( $W=-1$ ) for which $k$ increases with increasing $\alpha$. For each set, if one fixes the magnetic flux and examines the eigenstates, the scar repeats itself for $\Delta k \approx 2 \pi / L$. If there is no magnetic flux, the two sets of odd periodic scars intersect each other, leading to $\Delta k=\pi / L$ if the flow orientation is not distinguished. But if one regards the two sets as different scars, for each set the relation $\Delta k=2 \pi / L$ is recovered. For scars of even period, the two sets appear parallel to each other, i.e., they may appear for the same set of $k_{0}$ values with $2 \pi / L$ intervals, although for each value of $k_{0}$, typically only one scar can be found.

The wavevector $k$ for the scar decreases (increases) as $\alpha$ increases for $W=1(W=-1)$. The two lines must cross each other at certain points. For the period-3 scar, the cross points are $\alpha=0.25$ (corresponding to a $\pi$ phase difference) and $\alpha=0.75$. At the cross point, for some scars, it is difficult to identify the flow orientation. For the period-4-II scar, the cross points are at $\alpha=0$ and $\alpha=0.5$. For the period-3 scar, if $\alpha$ is shifted by 0.25 , then the $k$ - $\alpha$ relation will be similar to that for the period-4-II scar. That is, the behaviors of the period-3 scars for $\alpha=0.25$ are similar to those of the period-4-II scars for $\alpha=0$, and vice versa. A proper change in the external magnetic flux ( $\alpha=0.25)$ is thus able to switch the period-3 and period-4-II from being chiral to being non-chiral, and vice versa. This further elucidates the effect of the boundary induced phase $\beta$ : with reference to the period-4-II scar, $\beta$ shifts the overall pattern of the period- 3 scar leftwards from $\alpha=1 / 4$ to $\alpha=0$, with all other features remaining the same except that $k_{0}$ and $L$ take 
on different values. Similar behaviors were observed for scars of other periods and also for scars from a different system - the chaotic Africa Dirac AB billiard [124].

\section{E. Effects of symmetry operations on spin and chiral scars}

Symmetries and the spin characteristics are intimately related to each other, and play a pivotal role in the physical properties of quantum scars. For Dirac fermions, the following three types of symmetry (and their combinations) are fundamental: negative energy, negative potential and mirror symmetry.

\section{Negative energy $(-E)$ and positive potential $(V)$ with $V>E>0$}

Consider the action of the antiunitary operator $\hat{A}=\hat{\sigma}_{x} \hat{K}$ on $\hat{H}$

$$
\hat{H}^{\prime}=\hat{A} \hat{H} \hat{A}^{-1}=-\hat{H} \text {. }
$$

If $\Psi$ is an eigenstate (especially a scarred state) of $\hat{H}$, it can be transformed to

$$
\Psi^{\prime}=\hat{A}\left(\begin{array}{c}
\psi_{1} \\
\psi_{2}
\end{array}\right)=\left(\begin{array}{c}
\psi_{2}^{*} \\
\psi_{1}^{*}
\end{array}\right)
$$

which is also an eigenstate of $\hat{H}$ with energy $-E$ [93]. For the states corresponding to $E$ and $-E$ with the same potential $V$, the probability density distributions are identical: $\rho=\psi_{1}^{*} \psi_{1}+\psi_{2}^{*} \psi_{2}$. The in-plane probability density current is given by

$$
\boldsymbol{j}=c\langle\hat{\boldsymbol{\sigma}}\rangle=2 c\left[\operatorname{Re}\left(\psi_{1}^{*}(\boldsymbol{r}) \psi_{2}(\boldsymbol{r})\right), \operatorname{Im}\left(\psi_{1}^{*}(\boldsymbol{r}) \psi_{2}(\boldsymbol{r})\right)\right]
$$

It can be seen that the probability density current and the in-plane spin characteristics for negative energy $-E$ are the same as that for $E$ [Eq. (122)]. As a result, if $\Psi$ is a scarred state, the scarring currents associated with the $E$ and $-E$ states will be the same. Especially, according to Eq. (130), the condition $\hat{S}_{y} \Psi=(\hbar / 2) \Psi$ at the boundary $(V \rightarrow \infty)$ leads to $\hat{S}_{y} \Psi^{\prime}=(\hbar / 2) \Psi^{\prime}$. Further, the local expectation value of $\hat{\sigma}_{z}$ can be obtained. For the positive energy $(E)$ case, one has

$$
\left\langle\hat{\sigma}_{z}\right\rangle=\Psi^{\dagger} \hat{\sigma}_{z} \Psi=\psi_{1}^{*} \psi_{1}-\psi_{2}^{*} \psi_{2}
$$

The corresponding expression for the negative energy $(-E)$ case is

$$
\left\langle\hat{\sigma}_{z}\right\rangle=\Psi^{\prime \dagger} \hat{\sigma}_{z} \Psi^{\prime}=\psi_{2}^{*} \psi_{2}-\psi_{1}^{*} \psi_{1}
$$

The values of $\left\langle\hat{\sigma}_{z}\right\rangle$ have opposite signs for the $E$ and $-E$ cases. For $E>0$, one can get an explicit expression of the local average of $\left\langle\hat{\sigma}_{z}\right\rangle$ at the boundary interface with potential $V$ through Eqs. (124) and (129) as

$$
\left\langle\hat{\sigma}_{z}\right\rangle=\left(\Psi^{\mathrm{II}}\right)^{\dagger} \hat{\sigma}_{z} \Psi^{\mathrm{II}}=4 \cos ^{2} \gamma \frac{E q-V K}{(V-E)(q+K)} .
$$

It can be proven [124] that $\left\langle\hat{\sigma}_{z}\right\rangle \geq 0$. Especially, for $V \rightarrow+\infty$, one has $\left\langle\hat{\sigma}_{z}\right\rangle=0$. The counterpart expressions for $E<0$ are $\left\langle\hat{\sigma}_{z}\right\rangle \leq 0$ and $\left\langle\hat{\sigma}_{z}\right\rangle=0$ for $V \rightarrow+\infty$. 


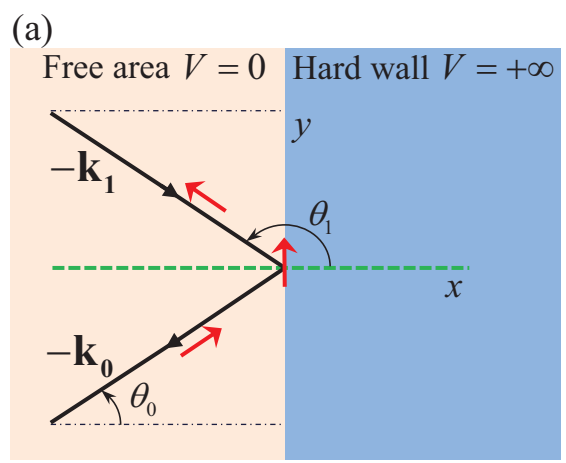

(b)

(c)

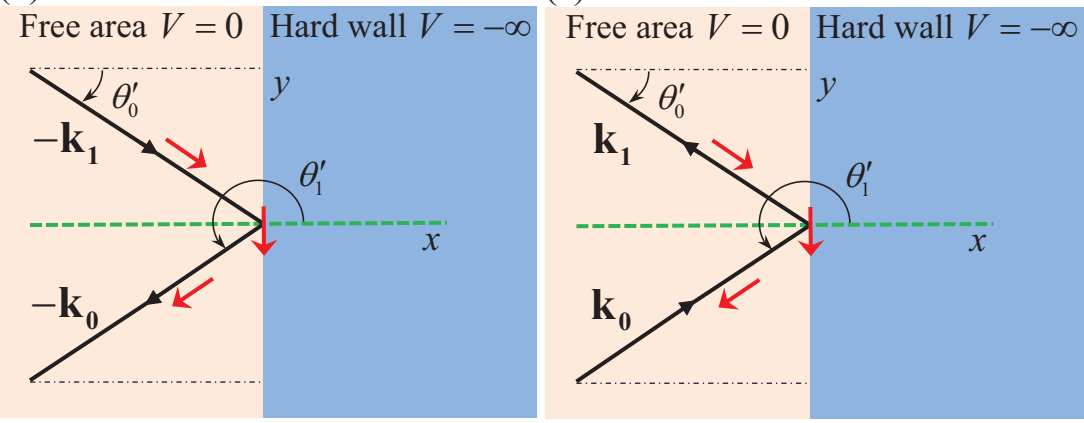

FIG. 34. Spin characteristics for positive and negative energies. Incident and reflected plane waves (black arrow) and the spin (red vertical arrow) corresponding to the superposition of waves at the boundary: (a) $-E, V$, and $V \rightarrow+\infty$, (b) $E,-V$, and $V \rightarrow+\infty$, and (c) $-E,-V$, and $V \rightarrow+\infty$. From Ref. [124] with permission.

It is insightful to investigate the spin characteristics at the boundary and to compare the accumulated phase difference of the scarred orbits with respect to the positive and negative energies by employing the plane wave model [Eqs. (123-126)]. For a free particle, the helicity is given by $\boldsymbol{\sigma} \cdot \boldsymbol{p} /|\boldsymbol{p}|=1$ and $\boldsymbol{\sigma} \cdot \boldsymbol{p} /|\boldsymbol{p}|=-1$, respectively, for the $E$ and $-E$ states, which indicates that, although the current orientation is the same for the $E$ and $-E$ states, the momentum of the free particle is in the reversed current direction for the $-E$ state. The wavefunction in the free region for a negative energy state is

$$
\Psi^{\mathrm{I}}=\frac{1}{\sqrt{2}}\left[\left(\begin{array}{c}
\exp \left\{-\frac{1}{2} i\left(\theta_{0}\right)\right\} \\
\exp \left\{\frac{1}{2} i\left(\theta_{0}\right)\right\}
\end{array}\right) \exp \left\{-i \boldsymbol{k}_{0} \cdot \boldsymbol{r}\right\}+R\left(\begin{array}{c}
\exp \left\{-\frac{1}{2} i\left(\theta_{1}\right)\right\} \\
\exp \left\{\frac{1}{2} i\left(\theta_{1}\right)\right\}
\end{array}\right) \exp \left\{-i \boldsymbol{k}_{1} \cdot \boldsymbol{r}\right\}\right]
$$

as illustrated in Fig. 34(a), where $\theta_{0}$ and $\theta_{1}$ are the spin directions of the free particle, and $\theta_{0}+\pi$ and $\theta_{1}+\pi$ are the corresponding wavevector directions. The transmitted wave in the potential region is

$$
\Psi^{\mathrm{II}}=\frac{T}{\sqrt{2}}\left(\begin{array}{c}
-i \chi_{1} \\
\chi_{2}
\end{array}\right) e^{-q x} e^{i K y}
$$

where the wavevectors are

$$
\begin{aligned}
& -\boldsymbol{k}_{0}=\left(-k \cos \theta_{0},-k \sin \theta_{0}\right) \text { and } \\
& -\boldsymbol{k}_{1}=\left(-k \cos \theta_{1},-k \sin \theta_{1}\right) .
\end{aligned}
$$


Other quantities are

$$
\begin{aligned}
K & =-k \sin \theta_{0}, \\
q & =\sqrt{\frac{V^{2}-E^{2}}{\hbar^{2} c^{2}}+K^{2},} \\
E & =\hbar c k, \\
\chi_{1} & =\sqrt{\frac{(V-E)(q-K)}{V q+E K}}, \text { and } \\
\chi_{2} & =\sqrt{\frac{(V+E)(q+K)}{V q+E K}} .
\end{aligned}
$$

Using the convention Eq. (125) and matching the wavefunctions $\Psi^{\mathrm{I}}$ and $\Psi^{\mathrm{II}}$ at the boundary lead to the formulas for $R$ and $T$. Especially, in the $V \rightarrow+\infty$ limit, one has $R=1$. With these results, the spin orientation at the boundary can be verified using Eq. (125) with $R=1$ :

$$
\hat{S}_{y} \Psi^{\mathrm{I}}=\frac{\hbar}{2} \Psi^{\mathrm{I}}
$$

Thus the spin points to the positive $y$-axis direction at the boundary for the negative energy state for $V \rightarrow \infty$. Further, the phase change associated with a scarred state with counterclockwise current is

$$
\Delta_{+}=\frac{1}{2}\left(\theta_{N}-\theta_{0}\right)=m \pi
$$

where $m$ is an integer. Comparing Eq. (148) with Eq. (134), one can see that the accumulated phase of the two orbits with the same current orientation for the $E$ and $-E$ states are identical. For the reversed orbit with a clockwise flow, the incident and reflected angles are defined as $\theta_{0}^{\prime}$ and $\theta_{1}^{\prime}$, which are the same as those defined in Fig. 30. Using Eqs. (131) and (132), one obtains the phase change

$$
\Delta_{-}=\frac{1}{2}\left(\theta_{n}^{\prime}-\theta_{0}^{\prime}\right)=-m \pi+N \pi
$$

where $N$ is the number of reflections along the orbit. This is the same as Eq. (135), so the accumulated phase difference between the reversed orbits caused by the boundary for the $-E$ state is

$$
\Delta_{+}-\Delta_{-}=2 m \pi-N \pi
$$

For an orbit with an odd number of bounces, there is an additional $\pi$ difference between the counterclockwise state and the clockwise state, so chiral scars can still emerge.

2. Positive energy (E), negative potential $(-V)$ and $V>E>0$

The time reversal operator is given by $\hat{\mathcal{T}}=i \hat{\sigma}_{y} \hat{K}$. Under the action of $\hat{\mathcal{T}}, \hat{H}$ is transformed to

$$
\hat{H}^{\prime}=\hat{\mathcal{T}} \hat{H} \hat{\mathcal{T}}^{-1}=c \hat{\boldsymbol{\sigma}} \cdot \hat{\boldsymbol{p}}-V(\boldsymbol{r}) \hat{\sigma}_{z},
$$


and the eigenstate $\Psi$ of $\hat{H}$ is transformed to

$$
\Psi^{\prime}=\hat{\mathcal{T}}\left(\begin{array}{c}
\psi_{1} \\
\psi_{2}
\end{array}\right)=\left(\begin{array}{c}
\psi_{2}^{*} \\
-\psi_{1}^{*}
\end{array}\right)
$$

where $\hat{H}^{\prime} \Psi^{\prime}=E \Psi^{\prime}$. The probability distribution is thus the same for $\Psi$ and $\Psi^{\prime}: \rho=\psi_{1}^{*} \psi_{1}+\psi_{2}^{*} \psi_{2}$, while the current orientations are opposite:

$$
\boldsymbol{j}^{\prime}=-2 c\left[\operatorname{Re}\left(\psi_{1}^{*}(\boldsymbol{r}) \psi_{2}(\boldsymbol{r})\right), \operatorname{Im}\left(\psi_{1}^{*}(\boldsymbol{r}) \psi_{2}(\boldsymbol{r})\right)\right]=-\boldsymbol{j} .
$$

The local expectation value of $\left\langle\hat{\sigma}_{z}\right\rangle$ is

$$
\left\langle\hat{\sigma}_{z}\right\rangle=\Psi^{\prime \dagger} \hat{\sigma}_{z} \Psi^{\prime}=\psi_{2}^{*} \psi_{2}-\psi_{1}^{*} \psi_{1}
$$

which is the same as that for the $(-E, V)$ case [Eq. (143)] and opposite to the $(E, V)$ case [Eq. (142)].

The spin orientation at the boundary in the plane-wave framework can now be examined, enabling the accumulated phase along the periodic orbit in the negative potential billiard to be calculated. The spinor wavefunction in the potential-free region is

$$
\Psi^{\mathrm{I}}=\frac{1}{\sqrt{2}}\left[\left(\begin{array}{c}
\exp \left\{-\frac{1}{2} i\left(\theta_{0}^{\prime}\right)\right\} \\
\exp \left\{\frac{1}{2} i\left(\theta_{0}^{\prime}\right)\right\}
\end{array}\right) \exp \left\{-i \boldsymbol{k}_{1} \cdot \boldsymbol{r}\right\}+R\left(\begin{array}{c}
\exp \left\{-\frac{1}{2} i\left(\theta_{1}^{\prime}\right)\right\} \\
\exp \left\{\frac{1}{2} i\left(\theta_{1}^{\prime}\right)\right\}
\end{array}\right) \exp \left\{-i \boldsymbol{k}_{0} \cdot \boldsymbol{r}\right\}\right],
$$

as illustrated in Fig. 34(b). The transmitted wave in the potential region has the form

$$
\Psi^{\mathrm{II}}=\frac{T}{\sqrt{2}}\left(\begin{array}{c}
i \chi_{1} \\
\chi_{2}
\end{array}\right) e^{-q x} e^{i K y}
$$

where the incident and reflected wavevectors are

$$
\begin{aligned}
& -\boldsymbol{k}_{1}=\left(k \cos \theta_{0}^{\prime}, k \sin \theta_{0}^{\prime}\right) \text { and } \\
& -\boldsymbol{k}_{0}=\left(k \cos \theta_{1}^{\prime}, k \sin \theta_{1}^{\prime}\right),
\end{aligned}
$$

respectively. Other parameters are

$$
\begin{aligned}
K & =k \sin \theta_{0}^{\prime}, \\
q & =\sqrt{\frac{V^{2}-E^{2}}{\hbar^{2} c^{2}}+K^{2},} \\
\chi_{1} & =\sqrt{\frac{(V-E)(q-K)}{V q+E K}}, \text { and } \\
\chi_{2} & =\sqrt{\frac{(V+E)(q+K)}{V q+E K}} .
\end{aligned}
$$

Matching the waves at the boundary and using the specularity Eq. (125) lead to formulas for the reflection and transmission coefficients. In particular, for $V \rightarrow-\infty$, one has $R=-1$. Since a $\pi$ phase in the reflection wave can reverse the spin orientation, at the boundary it is still an eigenfunction of $\hat{S}_{y}$ but with the eigenvalue $-\hbar / 2$, i.e.,

$$
\hat{S}_{y} \Psi=-\frac{\hbar}{2} \Psi
$$


regardless of the incident angle. From Eq. (135), one gets the whole phase change caused by the boundary along the clockwise orientation as

$$
\Delta_{-}=\frac{1}{2}\left(\theta_{n}^{\prime}-\theta_{0}^{\prime}\right)-N \pi=-m \pi,
$$

whereas the phase change along the counterclockwise direction is

$$
\Delta_{+}=\frac{1}{2}\left(\theta_{N}-\theta_{0}\right)-N \pi=m \pi-N \pi
$$

Because of the identity $\Delta_{+}-\Delta_{-}=2 m \pi-N \pi$, the chirality of the orbits with an odd number of bounces still exists in the negative potential case. In addition, a comparison between Eqs. (157) and (134) indicates that the difference in the accumulated phase of the two orbits with opposite current orientation corresponding to $(E, V)$ and $(E,-V)$ is an integer multiple of $2 \pi$.

\section{Negative energy $(-E)$, negative potential $(-V)$ and $V>E>0$}

Applying the unitary operator $\hat{U}=i \hat{\sigma}_{y} \hat{\sigma}_{x}$, which is a combination of two antiunitary operators $\hat{\sigma}_{x} \hat{K}$ and $i \hat{\sigma}_{y} \hat{K}$, to the Hamiltonian, one gets

$$
\hat{H}^{\prime \prime}=\hat{A} \hat{H} \hat{A}^{-1}=-\left(c \hat{\boldsymbol{\sigma}} \cdot \hat{\boldsymbol{p}}-V \hat{\sigma}_{z}\right),
$$

and the eigenstate $\Psi$ of $\hat{H}$ is changed to

$$
\Psi^{\prime}=\hat{U}\left(\begin{array}{c}
\psi_{1} \\
\psi_{2}
\end{array}\right)=\left(\begin{array}{c}
\psi_{1} \\
-\psi_{2}
\end{array}\right)
$$

Thus, $\Psi^{\prime}$ is the eigenstate of $\hat{H}^{\prime}=c \hat{\boldsymbol{\sigma}} \cdot \hat{\boldsymbol{p}}-V \hat{\sigma}_{z}$ associated with negative energy $-E$. The probability is still the same as that for the $(E, V)$ case, i.e., $\rho=\psi_{1}^{*} \psi_{1}+\psi_{2}^{*} \psi_{2}$, while the current orientation is reversed:

$$
\boldsymbol{j}^{\prime}=-2 c\left[\operatorname{Re}\left(\psi_{1}^{*}(\boldsymbol{r}) \psi_{2}(\boldsymbol{r})\right), \operatorname{Im}\left(\psi_{1}^{*}(\boldsymbol{r}) \psi_{2}(\boldsymbol{r})\right)\right]=-\boldsymbol{j} .
$$

The local expectation value of $\hat{\sigma}_{z}$ is

$$
\left\langle\hat{\sigma}_{z}\right\rangle=\Psi^{\prime \dagger} \hat{\sigma}_{z} \Psi^{\prime}=\psi_{1}^{*} \psi_{1}-\psi_{2}^{*} \psi_{2}
$$

which indicates that $\left\langle\hat{\sigma}_{z}\right\rangle$ is the same as that for the $(E, V)$ case.

The spin characteristics at the boundary can be confirmed and the global phase change of spin along a complete periodic orbit can be calculated through the plane wave model with the wave in the free area written as

$$
\Psi^{\mathrm{I}}=\frac{1}{\sqrt{2}}\left[\left(\begin{array}{c}
\exp \left\{-\frac{1}{2} i\left(\theta_{0}^{\prime}\right)\right\} \\
\exp \left\{\frac{1}{2} i\left(\theta_{0}^{\prime}\right)\right\}
\end{array}\right) \exp \left\{i \boldsymbol{k}_{1} \cdot \boldsymbol{r}\right\}+R\left(\begin{array}{c}
\exp \left\{-\frac{1}{2} i\left(\theta_{1}^{\prime}\right)\right\} \\
\exp \left\{\frac{1}{2} i\left(\theta_{1}^{\prime}\right)\right\}
\end{array}\right) \exp \left\{i \boldsymbol{k}_{0} \cdot \boldsymbol{r}\right\}\right] .
$$

A schematic diagram is shown in Fig. 34(c). The transmitted wave in the potential region has the form

$$
\Psi^{\mathrm{II}}=\frac{T}{\sqrt{2}}\left(\begin{array}{c}
i \chi_{1} \\
\chi_{2}
\end{array}\right) e^{-q x} e^{i K y}
$$


where the wavevectors are

$$
\begin{aligned}
& \boldsymbol{k}_{0}=\left(-k \cos \theta_{0}^{\prime},-k \sin \theta_{0}^{\prime}\right) \text { and } \\
& \boldsymbol{k}_{1}=\left(-k \cos \theta_{1}^{\prime},-k \sin \theta_{1}^{\prime}\right)
\end{aligned}
$$

Other parameters are

$$
\begin{aligned}
K & =-k \sin \theta_{0}^{\prime}, \\
q & =\sqrt{\frac{V^{2}-E^{2}}{\hbar^{2} c^{2}}+K^{2},} \\
\chi_{1} & =\sqrt{\frac{(V+E)(q-K)}{V q-E K}}, \text { and } \\
\chi_{2} & =\sqrt{\frac{(V-E)(q+K)}{V q-E K}} .
\end{aligned}
$$

Matching the wavefunctions at the boundary and using the specularity Eq. (125) lead to $R=-1$ for $V \rightarrow \infty$. The whole phase change caused by the boundary in the clockwise direction is

$$
\Delta_{-}=\frac{1}{2}\left(\theta_{N}^{\prime}-\theta_{0}^{\prime}\right)-N \pi=-m \pi
$$

which is the same as Eq. (157) for the $(E,-V)$ case. The accumulated phase along the counterclockwise direction is

$$
\Delta_{+}=\frac{1}{2}\left(\theta_{N}-\theta_{0}\right)-N \pi=m \pi-N \pi,
$$

which is identical to Eq. (158). Orbits with an odd number of bounces can still be chiral for the $(-E,-V)$ case. Comparing Eq. (166) with Eq. (134), one can see that the difference in the accumulated phase of the two orbits with the opposite current orientation for the $(-E,-V)$ and $(E, V)$ cases is still an integer multiple of $2 \pi$.

(a)

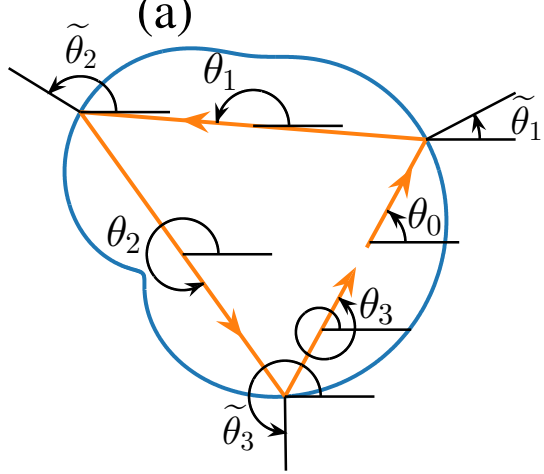

(b)

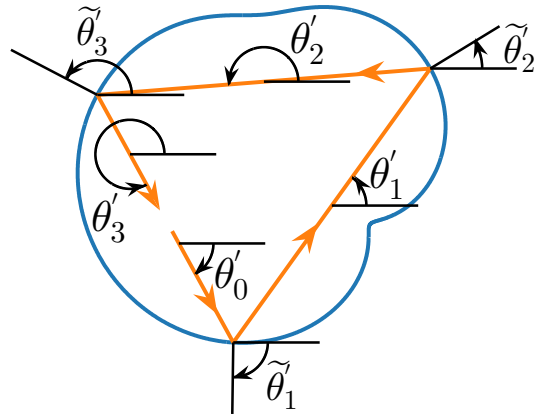

FIG. 35. A scarred orbit in the chaotic AB Africa Dirac billiard under mirror reflection. Africa-shape billiard with a counterclockwise flow: (a) the original scarred orbit, (b) the state under mirror reflection $\hat{A}=\hat{R}_{x} \hat{K}$. From Ref. [124] with permission. 


\section{Mirror reflection symmetry}

Under mirror reflection with respect to $y$-axis, the potential $V(x, y)$ changes to $V(-x, y)$ (under the action of antiunitary operator $\left.\hat{A}=\hat{R}_{x} \hat{K}\right)$, i.e.,

$$
\hat{H}^{\prime}=\hat{A} \hat{H} \hat{A}^{-1}=c \hat{\boldsymbol{\sigma}} \cdot \hat{\boldsymbol{p}}+V(-x, y) \hat{\sigma}_{z},
$$

as illustrated in Fig. 35. The eigenstate $\Psi$ of $\hat{H}$ is transformed to

$$
\Psi^{\prime}=\hat{R}_{x} \hat{K}\left(\begin{array}{c}
\psi_{1}(x, y) \\
\psi_{2}(x, y)
\end{array}\right)=\left(\begin{array}{c}
\psi_{1}^{*}(-x, y) \\
\psi_{2}^{*}(-x, y)
\end{array}\right)
$$

where $\hat{H}^{\prime} \Psi^{\prime}=E \Psi^{\prime}$. The probability distribution is

$$
\rho=\psi_{1}^{*}(-x, y) \psi_{1}(-x, y)+\psi_{2}^{*}(-x, y) \psi_{2}(-x, y),
$$

which is symmetric about the $y$ axis. The local current is

$$
\begin{array}{r}
\boldsymbol{j}^{\prime}=2 c\left[\operatorname{Re}\left(\psi_{1}(-x, y) \psi_{2}^{*}(-x, y)\right), \operatorname{Im}\left(\psi_{1}(-x, y) \psi_{2}^{*}(-x, y)\right)\right] \\
=2 c\left[\operatorname{Re}\left(\psi_{1}^{*}(-x, y) \psi_{2}(-x, y)\right),-\operatorname{Im}\left(\psi_{1}^{*}(-x, y) \psi_{2}(-x, y)\right)\right] .
\end{array}
$$

Thus one has $j_{x}^{\prime}(x, y)=j_{x}(-x, y)$, and $j_{y}^{\prime}(x, y)=-j_{y}(-x, y)$. This is quite surprising because, although the system is under the mirror reflection with respect to the $y$-axis, the current behaves as if it were under a parity operation with respect to the $x$-axis. This indicates that the currents associated with the scarred states with the same energy are in the same winding orientation, as shown in Fig. 35. The accumulated phase difference about a complete periodic orbit of the two systems with the same winding direction can be obtained, as follows. First, for odd orbits of the system with Hamiltonian $\hat{H}$ as shown in Fig. 35(a), the accumulated phase is [93]

$$
\Delta_{o}=\frac{1}{2}\left(\theta_{N}-\theta_{0}\right)=\frac{1}{2}\left(\pi-2 \theta_{0}+2\left(\sum_{j=1}^{M} \widetilde{\theta}_{2 j-1}-\sum_{j=1}^{M-1} \widetilde{\theta}_{2 j}\right)\right)
$$

where $M=(N+1) / 2$. While for the system $\hat{H}^{\prime}$ as illustrated in Fig. 35(b), the accumulated phase along the complete orbit is

$$
\Delta_{o}^{\prime}=\frac{1}{2}\left(\theta_{N}^{\prime}-\theta_{0}^{\prime}\right)=\frac{1}{2}\left(-\pi+2 \theta_{0}-2\left(\sum_{j=1}^{M} \widetilde{\theta}_{2 j-1}-\sum_{j=1}^{M-1} \widetilde{\theta}_{2 j}\right)+4 m \pi\right),
$$

where the angle relations $\theta_{0}^{\prime}=-\theta_{0}$ and $\widetilde{\theta}_{2 j-1}=\pi-\widetilde{\theta}_{2(M-j+1)-1}+2 n \pi$ have been used. Closure means $\Delta_{o}=K \pi$ ( $K$ is integer). As a result, one obtains the accumulated phase difference between these two orbits as

$$
\Delta_{o}-\Delta_{o}^{\prime}=2 K \pi-2 m \pi,
$$

which is integer multiples of $2 \pi$. For the orbits with an even number of bounces, the accumulated phase for the system with the Hamiltonian $\hat{H}$ is

$$
\Delta_{e}=\frac{1}{2}\left(\theta_{N}-\theta_{0}\right)=\left(\sum_{j=1}^{M} \widetilde{\theta}_{2 j}-\widetilde{\theta}_{2 j-1}\right)
$$


where $M=N / 2$. While for the system $\hat{H}^{\prime}$, the relation $\widetilde{\theta}_{2 j-1}=-\widetilde{\theta}_{2(M-j+1)}+\pi+2 n \pi$ leads to

$$
\Delta_{e}^{\prime}=\frac{1}{2}\left(\theta_{N}^{\prime}-\theta_{0}^{\prime}\right)=\left(\sum_{j=1}^{M} \widetilde{\theta}_{2 j}-\widetilde{\theta}_{2 j-1}+2 m^{\prime} \pi\right) .
$$

The accumulated phase difference is still an integer multiple of $2 \pi$. However, if the current in Fig. 35(b) flows in the opposite direction as that in Fig. 35(a) and it is associated with a scar with an odd number of bounces, an additional $\pi$ phase difference will arise in comparison with the scarred state in Fig. 35(a). While for orbits with an even number of bounces, this $\pi$ phase difference does not appear.
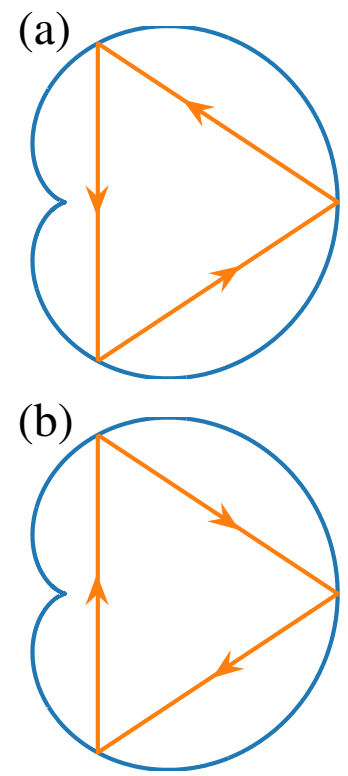

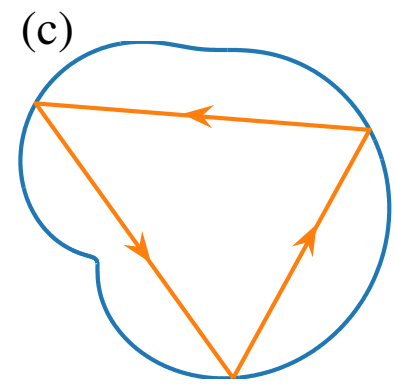

(d)

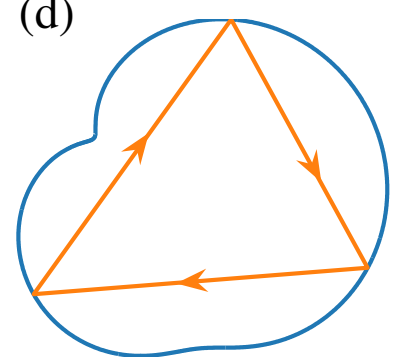

FIG. 36. Effects of parity operation on chiral scars. For the chaotic heart-shaped billiard [(a,b)] and Africa billiard [(c,d)] with certain flow orientation, (a) and (c): Scarring orbit of the original billiard at $(E, V(x, y))$. (b) and (d): Scarring orbit under the parity transformation $\left(\hat{\mathcal{P}}=\hat{R}_{y} \hat{\sigma}_{x}\right)$ at $(E,-V(x,-y))$. From Ref. [124] with permission.

\section{Parity operation}

The mirror symmetry reflection in Sec. IV E 4 can be further clarified by considering the parity operator with respect to the $x$ axis: $\hat{\mathcal{P}}=\hat{R}_{y} \hat{\sigma}_{x}$. Under its action, the Hamiltonian $\hat{H}$ is transformed to

$$
\hat{H}^{\prime}=\hat{P} \hat{H} \hat{P}^{-1}=c \hat{\boldsymbol{\sigma}} \cdot \hat{\boldsymbol{p}}-V(x,-y),
$$

which indicates that the parity operation is effectively equivalent to mirror reflection together with the time-reversal operation, which changes the sign of $V$. The eigenstate $\Psi$ of $\hat{H}$ becomes

$$
\Psi^{\prime}=\hat{\mathcal{P}}\left(\begin{array}{l}
\psi_{1}(x, y) \\
\psi_{2}(x, y)
\end{array}\right)=\left(\begin{array}{l}
\psi_{2}^{*}(x,-y) \\
\psi_{1}^{*}(x,-y)
\end{array}\right)
$$


where $\hat{H}^{\prime} \Psi^{\prime}=E \Psi^{\prime}$. After the parity operation, besides the mirror reflection with respect to the $x$ axis, the confinement potential changes sign, indicating that the parity symmetry is broken. The probability distribution is given by $\rho=\psi_{1}^{*}(x,-y) \psi_{1}(x,-y)+\psi_{2}^{*}(x,-y) \psi_{2}(x,-y)$, which is also symmetric about the $x$ axis. The local current is

$$
\begin{aligned}
\boldsymbol{j}^{\prime} & =2 c\left[\operatorname{Re}\left(\psi_{1}(x,-y) \psi_{2}^{*}(x,-y)\right), \operatorname{Im}\left(\psi_{1}(x,-y) \psi_{2}^{*}(x,-y)\right)\right] \\
& =2 c\left[\operatorname{Re}\left(\psi_{1}^{*}(x,-y) \psi_{2}(x,-y)\right),-\operatorname{Im}\left(\psi_{1}^{*}(x,-y) \psi_{2}(x,-y)\right)\right] .
\end{aligned}
$$

One thus has $j_{x}^{\prime}(x, y)=j_{x}(x,-y)$ and $j_{y}^{\prime}=-j_{y}(x,-y)$. Figure 36 shows a schematic diagram of the scarring current. For the chaotic heart-shaped billiard, one has $V(x, y)=V(x,-y)$, so the Hamiltonian $\hat{H}^{\prime}$ is invariant under the mirror reflection operation. This means that the time reversal operation $\hat{\mathcal{T}}$ [Eq. (151)] and the parity operation $\hat{\mathcal{P}}$ are equivalent for this system, and it is invariant under the combination of $\hat{\mathcal{P}}$ and $\hat{\mathcal{T}}$ operations:

$$
\hat{A}=\hat{R}_{y} \hat{\sigma}_{x} \cdot i \hat{\sigma}_{y} \hat{K}=-\hat{R}_{y} \hat{\sigma}_{z} \hat{K}
$$

For the chaotic Africa billiard, the scarring current orientation of $\hat{H}^{\prime}$ is opposite to that of the original scar. Rotation of the billiard in Fig. 36(d) by $\pi$ leads to the same geometric shape as the billiard in Fig. 35(b). The difference is in the sign of the potential, so the current directions are opposite for the two cases.

One can also use the parity operator $\hat{\mathcal{P}}^{\prime}=\hat{R}_{x} \hat{\sigma}_{y}$, which gives the parity operation with respect to the $y$ axis. The action of $\hat{\mathcal{P}}^{\prime}$ is equivalent to the combination of $\hat{\mathcal{P}}$ and a $\pi$ rotation. The mirror operator in fact is the combination of the parity and time-reversal operators:

$$
\hat{A}=\hat{R}_{x} \hat{K}=\hat{R}_{x} \hat{\sigma}_{y} \cdot \hat{\sigma}_{y} K
$$

6. Summary of spin characteristics of chaotic Dirac billiard under combinations of three basic symmetry operations

Similar analyses can be carried out [124] for all the combinations of $\pm E, \pm V$, with or without $\mathcal{M}$. The corresponding results are summarized in table V. Note that the normal time reversal operation $\hat{\mathcal{T}}$ corresponds to the case $(E,-V, \mathcal{I})$, and the parity operation $\hat{\mathcal{P}}$ is $(E,-V, \mathcal{M})$.

\section{F. Outlook and remarks on relativistic quantum scars}

While experimental observations of relativistic quantum scars in a mesoscopic graphene ring have been made recently [328], at the present there has been no experimental evidence for chiral scars in massless Dirac fermion systems. Fundamental to relativistic chiral scars is $\mathcal{T}$-symmetry breaking. Experimentally, the effects of such symmetry break can be investigated using topological insulators (TI). In particular, consider a 2D surface supporting the edge states of a 3D topological insulator, whose quasiparticles can be described by the $2 \mathrm{D}$ massless Dirac equation. Mass confinement can be realized by depositing a ferromagnet insulator cap layer on top of the TI outside of the billiard (or quantum dot) region [339-341], where the exchange coupling $V \hat{\sigma}_{z}$ induced by the ferromagnet insulator can serve as the mass confinement. A theoretical treatment usually requires an infinite mass potential. In realistic situations, insofar as the energies of the 
TABLE V. Characteristics associated with various combinations of three basic symmetry operations. Notations are the following: $E$ is the energy of the system, $V$ is the potential, $\mathcal{M}$ denotes mirror reflection with respect to the $y$-axis, $\mathcal{I}$ indicates the case without $\mathcal{M}$ operation, and $R$ is the plane wave reflection coefficient [Eq. (125)]. Helicity is defined as $\hat{\boldsymbol{\sigma}} \cdot \hat{\boldsymbol{p}} /|\boldsymbol{p}|$ in the free billiard domain $(V=0)$. If helicity is $1(-1)$, the direction of the wavevector is the same as (opposite to) the current orientation. Scarring current means the current orientation in the billiard domain with $+(-)$ indicating the parallel (opposite) orientation to that for the $(E, V, \mathcal{I})$ case. Spin orientation is the direction of spin at the boundary interface, where the + sign represents the positive $y$-direction (counterclockwise orientation with respect to the outer normal vector). The quantity $\left\langle\hat{\sigma}_{z}\right\rangle$ represents the local average spin in the $z$ direction at the boundary interface between a zero and a finite potential regions, and the $+(-)$ signs are for the positive (negative) $z$ axis. For $V \rightarrow \infty$, the average spin in the $z$ direction vanishes: $\left\langle\hat{\sigma}_{z}\right\rangle=0$.

\begin{tabular}{ccccccccc}
\hline \hline & $E$ & $E$ & $E$ & $E$ & $-E$ & $-E$ & $-E$ & $-E$ \\
& $V$ & $-V$ & $V$ & $-V$ & $V$ & $-V$ & $V$ & $-V$ \\
& $\mathcal{I}$ & $\mathcal{I}$ & $\mathcal{M}$ & $\mathcal{M}$ & $\mathcal{I}$ & $\mathcal{I}$ & $\mathcal{M}$ & $\mathcal{M}$ \\
\hline Reflection (R) & 1 & -1 & 1 & -1 & 1 & -1 & 1 & -1 \\
Helicity & 1 & 1 & 1 & 1 & -1 & -1 & -1 & -1 \\
Scarring current & + & - & + & - & + & - & + & - \\
Spin orientation & + & - & + & - & + & - & + & - \\
$\left\langle\hat{\sigma}_{z}\right\rangle$ & + & - & + & - & - & + & - & + \\
\hline \hline
\end{tabular}

concerned states are much smaller than the gap, the infinite potential approximation is valid. With respect to the application of the magnetic flux, the area through which the flux threads the surface can be finite, insofar as it is not on the orbit of any scar. Typical scars such as the period-3 and period-4 scars in Fig. 29 have a large open interior region, so they do not intersect a reasonably small flux region.

Through extensive computations and detailed physical analyses of the chaotic Dirac AB billiard systems [124], a relatively complete picture of the mechanism of $\mathcal{T}$-symmetry breaking emerges. For example, for the Dirac billiard confined by the infinite scalar four-potential, or mass potential, the Hamiltonian does not commute with the $\mathcal{T}$-operator, as the confinement mass potential will acquire a sign change after the $\mathcal{T}$-operation. This is corroborated by the fact that the boundary condition derived from the mass potential confinement does not commute with the $\mathcal{T}$-operator either. From the point of view of local physical interaction, each reflection at the boundary breaks the time-reversal symmetry as it contributes to an oriented flow at the boundary whose direction is independent of the incident angle. Further, as the spin of a free Dirac particle is polarized along its momentum, the reflection at the boundary induces a boundary-spin interaction. As a result, associated with each reflection an additional phase $\phi$ arises in the action integral of the particle. The reversed orbit acquires another phase $\widetilde{\phi}$ at this point. The phase difference between the counterclockwise reflection and its time reversed reflection at the same boundary point thus has a $\pi$ contribution. Consequently, for a scar on an orbit with an even number of reflections, these phases together contribute an integer multiple of $2 \pi$ to the phase difference of the counterclockwise orbit and its clockwise counterpart. For these orbits, the time-reversal symmetry is preserved. However, for the scars with an odd number of reflections, the boundary phases contribute an additional $\pi$, leading to the $\mathcal{T}$-symmetry breaking and making the scar chiral. 
The boundary-spin interaction induced phase can be compensated by a magnetic flux. The $\pi$ phase difference between the counterclockwise and clockwise orbits with an odd number of reflections can be annihilated completely by a proper flux amount. That is, a chiral scar can lose its chirality, while a non-chiral scar can attain the chirality with a suitable magnetic flux. Depending on the location of flux's threading the billiard, the winding number for an orbit about the flux can be highly nontrivial. For a given $\mathrm{AB}$ billiard, the winding numbers can be zero, one, two, and so on, and this has significant implications in the flux effect on scarring. Phenomenologically, the boundary induced phase can be included into the action integral, which is equivalent to the phase terms caused by the path integral of the momentum and, consequently, is equivalent to the phase from the magnetic flux.

Besides scars on periodic orbits, there is another class of states, the edge states, which always have a counterclockwise flow localized at the boundary. The edge states break the time-reversal symmetry as their time-reversed states are no longer solutions of the system. These states have nonzero wavefunctions at the boundary, in contrast to the case of a Schrödinger billiard system with an infinite confinement potential where the wavefunction must vanish at the boundary.

For the Dirac billiard system, chirality is fundamentally related to the time-reversal symmetry. The time-reversal operator changes the sign of the confinement potential $V$ and the direction of local flow for the scarred states. The parity operation is effectively a combination of time-reversal operation and mirror reflection. From a semiclassical point of view, for a particular scar, if the billiard has a reflection symmetry, e.g., the chaotic heart-shaped billiard, since the mirror reflection becomes the identity operation, the parity operation is equivalent to the time-reversal operation. As a result, if the system or the state is invariant under the parity operation, it will also be invariant under the time reversal operation. This occurs, for example, for scars of even periods in which the flow orientation can be either clockwise or counterclockwise for a given energy value. For scars with an odd number of bounces, both the parity and the time-reversal symmetries are broken, causing these scars to be chiral, for which only one orientation is allowed for a given energy level. For a billiard without a reflection symmetry (e.g., the chaotic Africa billiard), one can consider its mirror image. For scars on a given orbit, the corresponding scar under parity operation has the reverse orientation. These results can be generalized to more diverse physical situations, e.g., those with particle-hole symmetry, negative potential, mirror reflection and their combinations, where chirality can persist in spite of possibly different spin characteristics.

The comprehensive understanding of the $\mathcal{T}$-breaking of the system naturally leads to a control mechanism based on tuning the magnetic flux, by which the chiral nature of the scars can be manipulated, e.g., from chiral to non-chiral scars, and vice versa. The subtle $\mathcal{T}$-breaking effect associated with scarred states on odd periodic orbits and edge states can have significant implications in the transport behavior and spin textures of the relativistic pseudo-particles [339]. The distinct magnetic response can also be exploited for applications in quantum information devices, e.g., relativistic qubits [341]. Knowledge and advances in relativistic quantum chaos can be anticipated to have implications for a variety of applications of 2D Dirac materials. 


\section{RELATIVISTIC QUANTUM CHAOTIC SCATTERING}

\section{A. Quantum chaotic scattering - an introduction}

Classical chaotic scattering. Scattering is a process whereby particles are injected into a region of interaction, i.e., the scattering region, and exit after a finite amount of time. The characteristics of the particles after the scattering contain information about the interaction and the underlying system. Scattering is thus a fundamental tool to probe into the "inner gears" of physical and chemical systems. The goal of any scattering experiment is to obtain the scattering functions, which are the quantitative relations between output variables characterizing the particles after the scattering versus some input variables describing the particles before the scattering. For regular dynamics such as scattering from a single Coulomb potential, the scattering functions are smooth. About three decades ago, research in nonlinear dynamics revealed [36, 164-171, 173] that there can be relatively simple configurations, such as three potential hills positioned at the vertices of a triangle, in which the scattering functions can contain an uncountably infinite number of singularities that constitute a fractal set. About a singularity, a change in the input variable, no matter how small, can cause a large change in the output variable. This is precisely the hallmark of chaos - sensitive dependence on initial conditions, henceforth the term chaotic scattering [36, 164-171, 173].

From the standpoint of dynamics, chaotic scattering is due to the existence of nonattracting chaotic invariant sets (e.g., chaotic saddles) in the phase space. In a typical scattering scenario, incident particles move into the region in which a chaotic saddle resides along its stable manifold, stay in the vicinity of the saddle exhibiting chaotic behavior, and exit the region along the unstable manifold of the saddle. Because of the non-attracting nature of the chaotic saddle, any random incident trajectory can remain in the scattering region for a finite amount of time. This means that any chaotic behavior exhibited by the scattering trajectory is transient. In this sense, it is often said that chaotic scattering is the physical manifestation of transient chaos in classical Hamiltonian systems [171].

In the spectrum of distinct dynamical behaviors in Hamiltonian systems, one end is integrable dynamics that lead to regular scattering. The opposite end corresponds to hyperbolic dynamics that lead to fully developed chaotic scattering where all the periodic orbits are unstable [171]. In the parameter region in between these two end cases, nonhyperbolic chaotic scattering can arise where a nonattracting chaotic set coexists with Kolmogorov-Arnold-Moser (KAM) tori in the phase space. The main measurable difference between hyperbolic and nonhyperbolic chaotic scattering is that, for the former, the probability for the particles to stay in the scattering region decays exponentially but for the latter, the decay is algebraic as the particles can spend an arbitrarily long time in the vicinity of the KAM tori [342-347].

Quantum chaotic scattering. When describing the quantum-classical correspondence, it is convenient to regard the Planck constant as a control parameter, which is zero in the classical limit while it has a finite value in the quantum regime. Specifically, one can define $\hbar$ as the nondimensionalized Planck constant normalized by the characteristic length and momentum values of the system. There are then three regimes of interest: (1) the classical limit $\hbar \rightarrow 0$, (2) the semiclassical regime $\hbar \ll 1$, and (3) the fully quantum regime $\hbar \sim 1$. The semiclassical regime is of particular importance to quantum chaotic scattering - the study of quantum manifestations of classical chaotic scattering, because this is the regime where both quantum and classical effects are relevant. 
To characterize quantum scattering, one typically employs the scattering matrix (S-matrix), whose elements are the transition amplitudes between quantum states of the system before and after the scattering. It was shown more than four decades ago by Miller that the S-matrix can be expressed in terms of purely classical quantities [172]. For classical chaotic scattering, the Smatrix elements in the semiclassical regime exhibit random fluctuations $[173,348]$ with respect to variations in parameters such as the particle energy or the strength of an external magnetic field in a classically small but quantum-mechanically large range. In this regard, hyperbolic and nonhyperbolic scattering can have quite distinct quantum manifestations in the statistical properties of the fluctuations of the S-matrix elements [82]. Consider, for example, the strength of an S-matrix element versus the particle energy. For hyperbolic chaotic scattering, due to the exponential decay law in the classical limit, the autocorrelation function is Lorentzian with a zero derivative at zero energy increment [173, 349]. However, for nonhyperbolic scattering, due to the algebraic particle decay law in the classical limit, the autocorrelation function exhibits a cusp at zero energy increment [82]. As a result, small scale fluctuations of the S-matrix elements are characteristically greatly enhanced in the nonhyperbolic case as compared with the hyperbolic case.

\section{B. Conductance fluctuations associated with quantum transport and the relevance of quantum chaotic scattering}

Conductance fluctuations are a fundamental phenomenon in open quantum systems. An important result is universal conductance fluctuations (UCFs) in mesoscopic systems [80, 350-353]. The pioneering work of Lee and Stone [350] established theoretically that, for mesoscopic metal samples, when the inelastic diffusion length exceeds the sample dimensions, the conductance fluctuations are of the same order as the conductance quanta, which is independent of the sample size and of the degree of disorder at low temperatures, thereby giving rise to universal features. This result was consistent with both experimental measurements [351] and numerical simulations [353].

The anomalous transport behaviors of charge carriers in graphene systems are particularly relevant to relativistic quantum chaos. The phase coherent length of graphene at low temperatures can be as long as several hundred nanometers [354] or even micrometers [355]. It was demonstrated experimentally that for graphene quantum dots smaller than $100 \mathrm{~nm}$, the conductance peaks become strongly aperiodic, indicating a major contribution of the quantum confinement [356]. While it can be difficult to cut graphene precisely following a particular shape under $100 \mathrm{~nm}$, the irregularities of the boundary often drive the classical dynamics to the chaotic regime, yielding transport properties mimicking those of quantum chaotic scatterings and also Gaussian ensembles of the peak spacing distributions [356]. Conductance fluctuations in graphene systems have been studied experimentally and analyzed using the framework of UCFs [308, 357, 358]. Rycerz et al. [359] found theoretically that, for strong disorder, the fluctuation behaviors agree with the Altshuler-LeeStone prediction $[350,360]$. However, in the case of weak disorder, abnormally large conductance fluctuations (with magnitude several times larger than that in the strong disorder case) can occur, which can be attributed to the absence of backscattering due to the honeycomb lattice structure. Horsell et al. [361] subsequently found that the variance of UCFs in both monolayer and bilayer graphene flakes is strongly affected by elastic scattering, particularly, by inter-valley scattering, though the correlation of the fluctuations as a function of the Fermi energy is insensitive to the specific scattering mechanisms under common experimental conditions. For few-layer graphene flakes in contact with superconducting leads, conductance fluctuations can be enhanced if the ap- 
plied voltage is smaller than the superconducting energy gap [362].

The seminal work of Jalabert, Baranger, and Stone [80] suggested that conductance fluctuations in the ballistic regime can be a probe of quantum chaos, establishing for the first time a connection between quantum transport in solid-state devices and classical chaos. Subsequent works [81-88] revealed that UCFs are intimately related to the study of quantum chaotic scattering $[36,173]$. A result in nonrelativistic quantum chaotic scattering is that, for those with integrable or mixed (nonhyperbolic) classical dynamics, sharp conductance fluctuations can occur. This is because, in the corresponding classical phase space, there are KAM islands centered about stable periodic orbits, associated with which there is quantum mechanically little interaction between the corresponding bounded states and the electron waveguides (leads), giving rise to extremely sharp conductance fluctuations on energy scales of the same order of magnitude as the interaction energy [73]. The abrupt conductance changes are in fact a kind of Fano resonance [163, 363-366]. However, if the classical dynamics are chaotic, due to ergodicity of the classical orbits, the corresponding quantum states will have strong interactions with the leads regardless of their positions. As a result, there is little probability for localized states with long lifetime to form, leading to smooth conductance fluctuations over the energy scale determined by the interaction strength. The distinct types of classical dynamics thus have marked fingerprints in the quantum conductance fluctuation patterns, which can be exploited to modulate the conductance fluctuations in quantum dot devices by controlling the corresponding classical dynamics [127, 128]. Note that, a closed system exhibiting chaos in the classical limit is capable of generating scarred states in the quantum regime $[121,123,367,368]$. However, when the system is open, the degree of localization of the originally scarred states in a fully chaotic system is generally much weaker than that in classically integrable or nonhyperbolic systems.

\section{Relativistic quantum chaotic scattering in graphene quantum dots}

Relativistic quantum manifestations of classical chaotic scattering were studied initially using graphene quantum dots [369]. In particular, the electronic transport properties were investigated in open graphene quantum dots (GQDs) with both hyperbolic and nonhyperbolic scattering dynamics in the classical limit, which were compared with those in nonrelativistic quantum dot (NRQD) systems. For NRQD systems with nonhyperbolic chaotic scattering, it was demonstrated using semiclassical theory [83] and in simulations and experiments $[84,86,88,370]$ that the conductance exhibits fractal fluctuation patterns. An examination of the Wigner time delay and the resonance width of the conductance profile revealed that, while the distribution of the resonance width is algebraic as predicted semiclassically, the energy scale is in contrast far below the mean energy level-spacing [85].

Associated with transport through a quantum dot, narrow resonances can arise, which are caused by the weak coupling between the localized states about the stable periodic orbits and the leads $[371,372]$. The tunneling between the chaotic region and the stable KAM island was investigated numerically [373-375] and experimentally [87] with the striking finding that GQDs generally have sharper conductance fluctuations than NRQDs. Moreover, even for hyperbolic GQDs, pronounced quantum pointer states $[121,156]$ persist. The resonances associated with the transmission are well characterized by the Fano profile [363] with the width given by the imaginary part of the eigenenergies of the effective dot Hamiltonian, where the effects of the leads are theoretically described by the self-energies. 
(a)
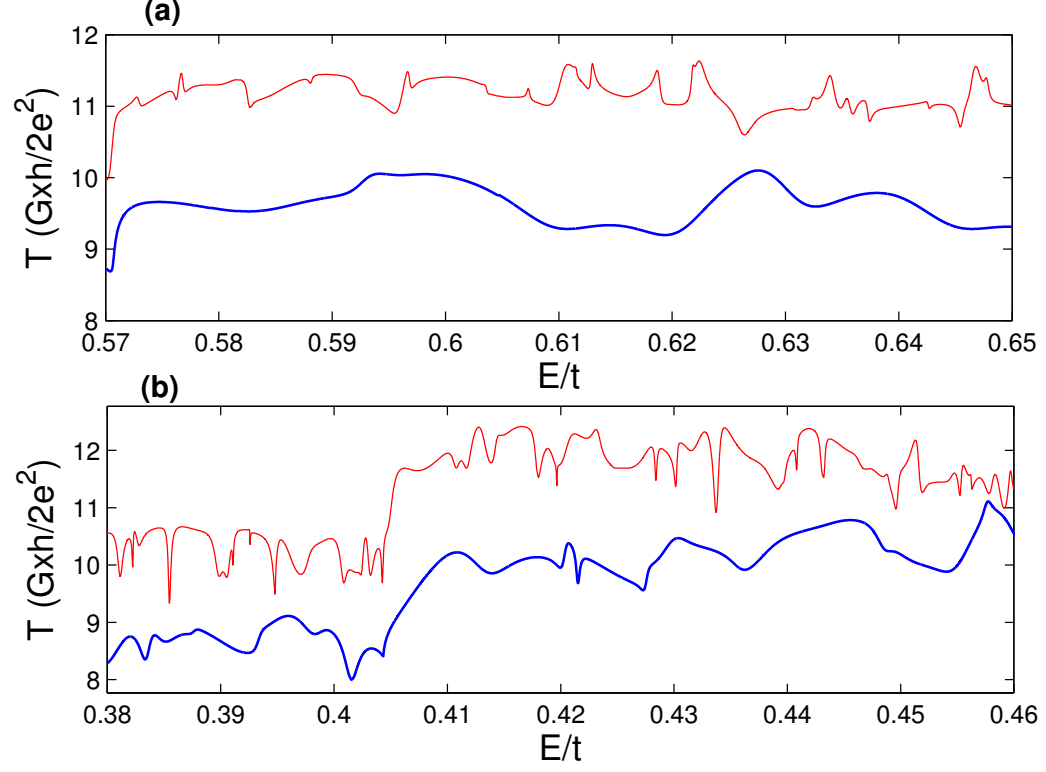

FIG. 37. Conductance fluctuations associated with transport through nonrelativistic and graphene quantum dots whose corresponding classical scattering dynamics can be either nonhyperbolic or hyperbolic. The notions NRQD and GQD stand for nonrelativistic quantum and graphene quantum dot, respectively. Shown are the transmission $T$ versus energy $E$ for (a) NRQD and (b) GQD. Red and blue curves are for nonhyperbolic and hyperbolic classical scattering dynamics, respectively. The quantum dots for the NRQD/nonhyperbolic, NRQD/hyperbolic, GQD/nonhyperbolic and GQD/hyperbolic combinations consist of 16611, 8401, 28864 and 14720 atoms, respectively. From Ref. [369] with permission.

To systematically investigate the quantum scattering dynamics in open graphene quantum dots, the class of open cosine billiards [85] capable of generating both hyperbolic and nonhyperbolic chaotic scattering dynamics through continuous parameter variation was chosen as a prototypical system. The open billiard is defined by two hard walls at $y=0$ and $y(x)=W+(M / 2)[1-$ $2 \cos (2 \pi x / L)]$, respectively, for $0 \leq x \leq L$, with two semi-infinite leads of width $W$ attached to the left and right openings of the billiard. Suitable adjustment of the ratios $W / L$ and $M / L$ can lead to changes in the stabilities of the classical periodic orbits, associated with which a transition from nonhyperbolic to hyperbolic chaotic scattering dynamics can occur. For example, for $W / L=0.18$ and $M / L=0.11$, there is nonhyperbolic scattering but for $W / L=0.36$ and $M / L=0.22$, the scattering dynamics become fully chaotic or hyperbolic [85].

In Ref. [369], the tight-binding approach and the Landauer-Büttiker formalism in combination with the non-equilibrium Green's function (NEGF) method [376, 377] were used to calculate the conductance/transmission and the local density of states (LDS), where all energies were expressed in units of the hopping energy $t$. In particular, the retarded Green's function is

$$
G(E)=\left(E \cdot I-H-\Sigma_{R}(E)-\Sigma_{L}(E)\right)^{-1},
$$

where $\Sigma$ is the retarded self-energy characterizing the effect of the lead, and the subscripts $R$ and $L$ indicate the right and left leads, respectively. The transmission $T$ is given by

$$
T(E)=\operatorname{Tr}\left[\Gamma_{L}(E) G(E) \Gamma_{R}(E) G^{\dagger}(E)\right],
$$


where $\Gamma_{L, R}(E)$ are the coupling matrices of the quantum dot for the left and right leads:

$$
\Gamma_{L, R}(E)=i\left[\Sigma_{L, R}(E)-\Sigma_{L, R}^{\dagger}(E)\right] .
$$

In the low temperature limit, the conductance can be calculated using the Landauer formula [377]:

$$
G \approx \frac{2 e^{2}}{h} T(E)
$$

The local density of states for the device can be obtained via

$$
\rho=-\frac{1}{\pi} \operatorname{Im}[\operatorname{diag}(G)]
$$

and the local current between the nearest neighbor lattice point $i$ and $j$ is given by

$$
J_{i \rightarrow j}=\frac{4 e}{h} \operatorname{Im}\left[H_{i j} C_{j i}^{n}(E)\right]
$$

where $C^{n}=G \Gamma_{L} G^{\dagger}$ is the electron correlation function and $H_{i j}$ is an element of the Hamiltonian matrix [377]. To improve the computational efficiency, the transmission, LDS and the local current can be calculated using the recursive Green's function method.

The transmission fluctuations were calculated [369] for the four combinations of quantum dots and classical scattering dynamics: NRQD/hyperbolic, NRQD/nonhyperbolic, GQD/hyperbolic, and GQD/nonhyperbolic. For comparison, all the dots studied have the same maximum number of propagating modes: $N_{\text {mode }}=48$, and GQDs have zigzag boundaries terminated in the horizontal direction (for NRQDs the shape of the boundary is not an issue). Typical patterns of the transmission fluctuations are shown in Fig. 37, where the energy ranges were chosen such that the transmissions for different cases have similar values. The results for NRQDs [Fig. 37(a)] are consistent with those from previous works, i.e., nonhyperbolic chaotic scattering in the classical limit leads to sharper quantum fluctuations. If the classical scattering dynamics are hyperbolic, the transmission varies much more smoothly with the energy [85]. Similar behaviors were observed for GQDs, as shown in Fig. 37(b). Comparing Figs. 37(b) and 37(a) reveals enhanced fluctuations in the graphene case, for both hyperbolic and nonhyperbolic chaotic scattering. Even in the hyperbolic case, the transmission associated with the GQD contains sharper resonances as compared with that in the NRQD. This suggests a stronger localization effect [371, 372] in the GQD.

The autocorrelation function calculated from the transmission-energy curve after removing the smooth background variation can be used to characterize the conductance fluctuations quantitatively. The results are shown in Fig. 38. As expected, for the GQDs, the correlation functions decay faster than those for NRQDs, indicating stronger fluctuations of the transmission or, equivalently, a smaller energy scale over which a large change in the transmission can occur. This is consistent with the result that GQDs tend to have enhanced conductance fluctuations in the presence of disorder, due to the absence of back scattering [359] or to the Andreev reflection at the graphene-superconductor interface [362].

The fluctuation patterns can be characterized and understood by the behaviors of the complex eigenvalues of the non-Hermitian Hamiltonian of the whole open system. In particular, when leads are not present so that the quantum-dot system is closed, the device Hamiltonian describing this closed system is Hermitian so that all eigenenergies are real. The effect of a semi-infinite lead, 


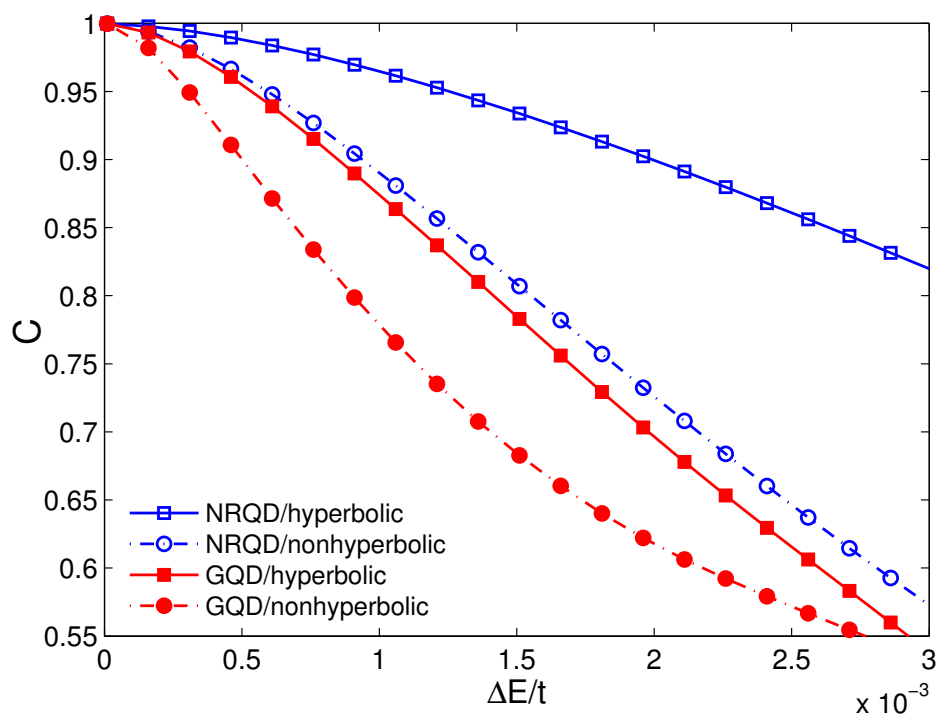

FIG. 38. Quantitative characterization of conductance fluctuations in NRQD and GQD with classically nonhyperbolic or hyperbolic chaotic scattering dynamics. Shown are curves of the autocorrelation $C$ versus the energy increment $\Delta E$ for different quantum dot structures in Fig. 37.

however, can be treated by constructing the corresponding self-energy through the non-equilibrium Green's function formalism [377]. When all the self-energy terms corresponding to multiple leads are combined and inserted in the device Hamiltonian, the resulting Hamiltonian is no longer Hermitian and typically possesses complex eigenenergies. It was demonstrated [130] that, even for the situation where there is no unique classical dynamics, the magnitude of the imaginary parts of the eigenenergies provides a reasonable characterization of the conductance-fluctuation patterns. A particular feature is that, when eigenvalues with extremely small imaginary parts emerge, sharp conductance fluctuations occur in a nearly abrupt fashion over small energy scales.

A theoretical explanation of the phenomena in Figs. 37 and 38 can then be obtained through examination of the Fano resonances with respect to the coupling between the eigenstates in the quantum dots and the leads. It was found [369] that the coupling is typically much weaker in the GQD than that in the NRQD for both the hyperbolic and nonhyperbolic systems. In particular, in the tight-binding framework, by considering the scattering region as a closed system with Hamiltonian matrix $H_{c}$, the effect of the leads can be treated using the retarded self-energy matrices, $\Sigma^{R}=\Sigma_{L}^{R}+\Sigma_{R}^{R}$. Note that $H_{c}$ is Hermitian with a set of real eigenenergies and eigenfunctions $\left\{E_{0 \alpha}, \psi_{0 \alpha} \mid \alpha=1, \cdots, N\right\}$, but $\Sigma^{R}(E)$ is in general not Hermitian and depends on the Fermi energy $E$. The effective Hamiltonian matrix $H_{c}+\Sigma^{R}(E)$ thus has a set of complex eigenenergies with the eigenfunctions

$$
\begin{aligned}
{\left[H_{c}+\Sigma^{R}(E)\right] \psi_{\alpha} } & =E_{\alpha} \psi_{\alpha}, \\
\phi_{\alpha}^{T}\left[H_{c}+\Sigma^{R}(E)\right] & =E_{\alpha}^{*} \phi_{\alpha}^{T},
\end{aligned}
$$

where

$$
E_{\alpha}=E_{0 \alpha}-\Delta_{\alpha}-i \gamma_{\alpha}
$$

The self-energy matrix $\Sigma^{R}$ has nonzero elements only in the subblock of the boundary atoms connecting with the leads. For most of the eigenstates, $\Sigma^{R}$ can be treated as a perturbation, thus 
$\Delta_{\alpha}$ and $\gamma_{\alpha}$ are generally small.

The Green's function matrix can be expanded as

$$
G^{R}(E)=\sum_{\beta}\left[\psi_{\beta}(E) \phi_{\beta}(E)^{\dagger}\right] /\left[E-E_{\beta}(E)\right]
$$

For a particular eigenstate $\left\{E_{\alpha}, \psi_{\alpha}\right\}$, for $E$ close to $E_{\alpha}, G^{R}$ can be rewritten as

$$
G^{R}=G_{0}^{R}(E)+G_{1}^{R}(E)
$$

where

$$
G_{0}^{R}(E)=\sum_{\beta \neq \alpha}\left[\psi_{\beta}(E) \phi_{\beta}(E)^{\dagger}\right] /\left[E-E_{\beta}(E)\right]
$$

varies slowly since $\left|E-E_{\beta}\right|$ is large and

$$
G_{1}^{R}(E)=\left[\psi_{\alpha}(E) \phi_{\alpha}(E)^{\dagger}\right] /\left[E-E_{\alpha}(E)\right]
$$

changes fast as $E$ is in the vicinity of $E_{\alpha}$. Since the self-energy $\Sigma^{R}$ is a slow variable, the coupling matrix

$$
\Gamma_{L, R}^{R}=i\left[\Sigma_{L, R}^{R}-\left(\Sigma_{L, R}^{R}\right)^{\dagger}\right]
$$

is slowly varying, too. Thus, in the expression of the transmission

$$
T=\operatorname{Tr}\left[\Gamma_{L}^{R} G^{R} \Gamma_{R}^{R}\left(G^{R}\right)^{\dagger}\right]
$$

the only fast variable is $G_{1}^{R}(E)$, while all others can be treated approximately as constants and evaluated at some arbitrary energy value $E_{0}$ close to $E_{\alpha}$. Choosing $E_{0}=E_{\alpha}$, the transmission in the vicinity of $E_{\alpha}$ can be approximated as

$$
T(E) \approx T_{0}\left(E_{0}\right)+\Delta T(1-2 q \varepsilon) /\left(\varepsilon^{2}+1\right),
$$

where

$$
\begin{aligned}
T_{0} & =\operatorname{Tr}\left[\Gamma_{L}^{R} G_{0}^{R} \Gamma_{R}^{R}\left(G_{0}^{R}\right)^{\dagger}\right], \\
\Delta T & =T\left(E_{0}\right)-T_{0}\left(E_{0}\right), \\
q & =\operatorname{Im}\left(\operatorname{Tr}\left[\Gamma_{L}^{R} G_{1}^{R} \Gamma_{R}^{R}\left(G_{0}^{R}\right)^{\dagger}\right]\right) / \Delta T, \\
\varepsilon & =\left(E-\operatorname{Re}\left(E_{\alpha}\right)\right) / \gamma_{\alpha} .
\end{aligned}
$$

One thus gets

$$
T(E) \approx T_{0}\left(E_{0}\right)-\Delta T+\Delta T \frac{(\varepsilon-q)^{2}}{\varepsilon^{2}+1}+\Delta T \frac{2-q^{2}}{\varepsilon^{2}+1} .
$$

This formula agrees with numerical results [369], which represents a generalized Fano resonance, and is consistent with results on Fano resonance profiles of conductance obtained from the scattering matrix elements $[372,378]$. Thus the transmission curve has a resonance at $\operatorname{Re}\left(E_{\alpha}\right)$, where the width is on the order of $\gamma_{\alpha}$. Since $\Sigma^{R}$ depends on the energy $E, \Delta_{\alpha}$ and $\gamma_{\alpha}$ are also functions of $E$. Thus the approximate theory is valid for eigenstates [377] whose values of $\operatorname{Re}\left(E_{\alpha}\right)$ are close to $E$. 
A physical understanding of the persistent sharp conductance fluctuations can be obtained through analyzing the resonance width, the localized states, and the local current patterns. For example, the transmission resonance is characterized by Fano profiles [364], where the width of the resonance can be related to the localization of the electronic states [128, 163, 371, 372]. A key to understanding the distinct characteristics of the transmission in NRQDs and GQDs with different types of chaotic scattering in the classical limit is the relation between the LDS patterns and the width of the resonances. For this purpose, the first-order approximation of $\gamma_{\alpha}$ can be calculated. In the absence of a magnetic field, $H_{c}$ is real symmetric, so $\left\{\psi_{0 \alpha} \mid \alpha=1, \cdots, N\right\}$ forms a set of orthogonal and complete basis. Generally, one has

$$
\psi_{\alpha}=\psi_{0 \alpha}-\delta_{r} \psi_{\alpha r}-i \delta_{i} \psi_{\alpha i}
$$

where $\delta_{r}$ and $\delta_{i}$ are small quantities. Substituting $E_{\alpha}$ and $\psi_{\alpha}$ into the eigenequation for the nonHermitian Hamiltonian $H_{c}+\Sigma^{R}$ underlying an open quantum dot system

$$
\left[H_{c}+\Sigma^{R}\right] \psi_{\alpha}=E_{\alpha} \psi_{\alpha}
$$

keeping only the first-order terms of the small quantities, and taking into account the orthogonality of $\psi_{0 \alpha}$, one has

$$
\Delta_{\alpha}+i \gamma_{\alpha} \approx-\left\langle\psi_{0 \alpha}\left|\Sigma^{R}\right| \psi_{0 \alpha}\right\rangle
$$

and, consequently,

$$
\gamma_{\alpha}=-\left\langle\psi_{0 \alpha}\left|\operatorname{Im}\left(\Sigma^{R}\right)\right| \psi_{0 \alpha}\right\rangle,
$$

which indicates that the width of the transmission resonance $\left(\gamma_{\alpha}\right)$ is determined by the imaginary part of the self-energy $\Sigma^{R}$ and the corresponding wave function $\psi_{0 \alpha}$ of the closed system. Because $\Sigma^{R}$ has nonzero elements only at the boundary atoms connecting with the leads, only the values of $\psi_{0 \alpha}$ on the same set of atoms contribute to $\gamma_{\alpha}$. Since the wave function is normalized, localized states that assume large values on a subset of atoms, say, atoms on a particular stable orbit, will have small values on the boundary atoms, resulting in small values of $\gamma_{\alpha}$. For dispersive states where $\psi_{0 \alpha}$ takes on similar values on all atoms, the values on the boundary atoms are of the order of $1 / \sqrt{N}$. Thus $\gamma_{\alpha}$ depends mainly on $\Sigma^{R}$. For cases of identical leads, one has $\gamma_{\alpha} \sim 1 / N$. Nonhyperbolic quantum dots have about twice the number of atoms as the hyperbolic QDs, so $\gamma_{\alpha}$ is about half the value, which was verified numerically [369]. The system-size caused effect only modulates the results by a factor of 2 , while the features of localization can contribute to the difference in $\gamma_{\alpha}$ by several orders of magnitude. Since the eigen-wavefunction is highly correlated with the LDS patterns, the explanation is valid for LDS patterns, or pointer states.

The approximate analysis of the transmission resonance requires $\gamma_{\alpha}$ to be much smaller than the spacing between the adjacent energy levels, i.e., for separated and localized states. For large values of $\gamma_{\alpha}$, the resonances are broadened and it is difficult to distinguish them from the background variations. This was the reason that, for NRQDs with nonhyperbolic scattering dynamics, a similar analysis can be valid but only when strong localizations on stable periodic orbits occur [372]. For GQDs, computations revealed [369] sharp conductance resonances (Figs. 37 and 38) for both hyperbolic and nonhyperbolic classical scattering dynamics, leading to small values of $\gamma_{\alpha}$. Figure 39 shows the eigenenergies $E_{\alpha}$ in the complex plane in a proper energy range. The energy for which the self-energy matrix is evaluated is $E_{0}=0.2 t$ for GQDs and $E_{0}=t$ for NRQDs. In principle, the plots are accurate only for the eigenenergies whose $\operatorname{Re}\left(E_{\alpha}\right)$ values are close to $E_{0}$. However, even if $\operatorname{Re}\left(E_{\alpha}\right)$ is far from $E_{0}$, it remains to be a good approximation [369]. For a 

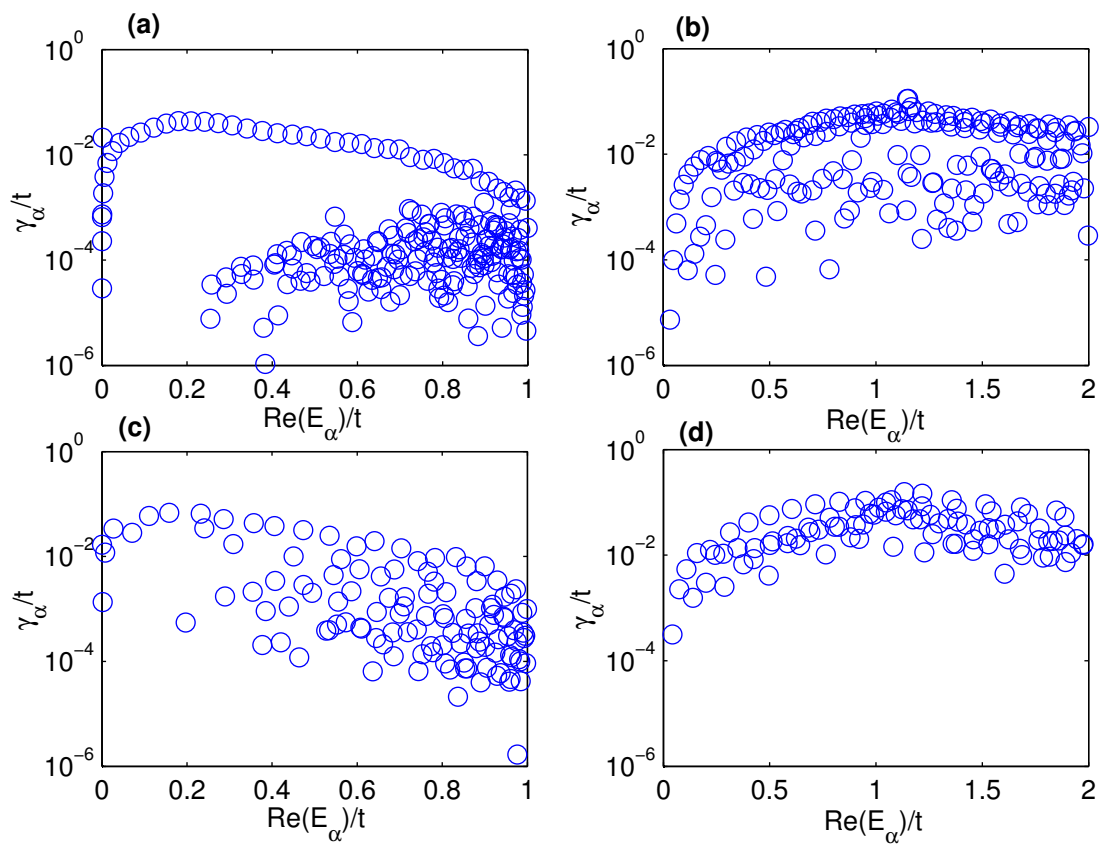

FIG. 39. Physical explanation of emergence of Fano resonance through the eigenvalues of nonHermitian Hamiltonian for the open quantum dot systems. Shown are the plot of imaginary parts $\gamma$ versus real parts of the eigenenergies $E_{\alpha}$ for (a) GQD/nonhyperbolic, (b) NRQD/nonhyperbolic, (c) GQD/hyperbolic, and (d) NRQD/hyperbolic. The energy values $E_{0}$ in (a-d) are $0.2,1,0.2$ and 1, respectively. From Ref. [369] with permission.

larger system, there are more data points in the plots, but the distribution of the points remains the same. These results verified those in Fig. 38 in that the correlation of the conductance fluctuations of the system with a smaller $\gamma_{\alpha}$ value decays faster. From Fig. 39, one can see that, the four cases have the common feature that they all possess a continuous line shape about $\gamma_{\alpha} \sim 10^{-2} t$. These values contribute to the conductance variations on energy scales of $10^{-2} t$ to $10^{-1} t$ and hence to the smooth background. For NRQDs, the hyperbolic case has only this part, but the nonhyperbolic case also has relatively lower values in the range $10^{-4} t$ to $10^{-2} t$ [Fig. 39(b)], which correspond to the localized states. The separation between the two parts is not sharp, due to the heterogeneous, mixed phase-space structure associated with nonhyperbolic chaotic scattering [371]. For GQDs, for both the hyperbolic and nonhyperbolic cases, the distributions of the eigenenergies contain two parts: one with and another without localized states. For the nonhyperbolic case, the two parts are well separated and the lower part is several orders of magnitude smaller than that associated with the nonhyperbolic NRQD, as shown in Fig. 39(a), indicating much sharper transmission fluctuations. Furthermore, the hyperbolic GQD also contains such a lower part [Figs. 39(c)], providing an explanation for the observed sharp resonances in Fig. 37(b). While the classical scattering dynamics are purely chaotic and the quantum manifestations are expected of those situations where there is no strong localization, the same quantum dot filled with graphene exhibits characteristically different behavior.

The transport fluctuations for GQDs and NRQDs that exhibit both hyperbolic and nonhyperbolic chaotic scattering in the classical limit thus exhibit distinct features. In particular, for each type of quantum dots, the one with classical nonhyperbolic scattering dynamics exhibits enhanced 


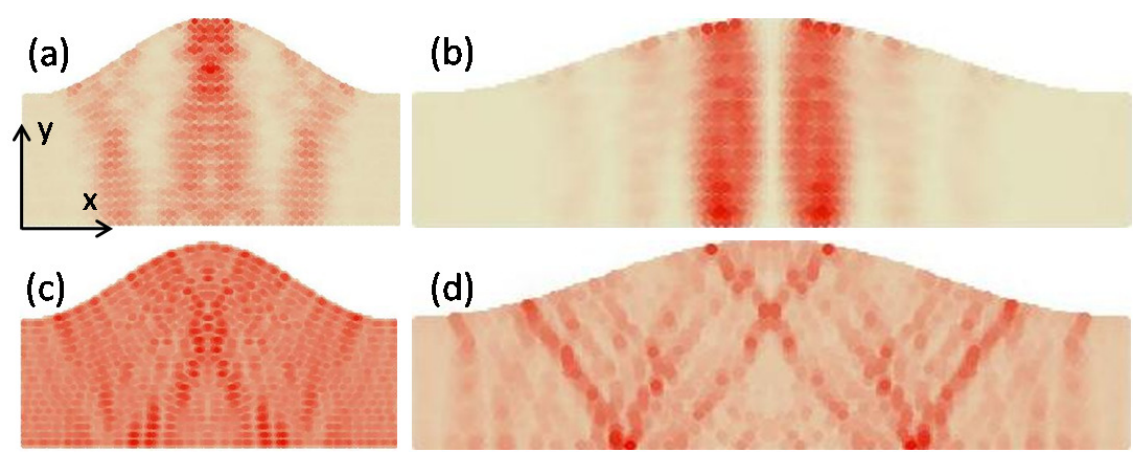

FIG. 40. Quantum pointer states as a cause of various transmission resonances. Quantum pointer states for (a) GQD/hyperbolic, (b) GQD/nonhyperbolic, (c) NRQD/hyperbolic, and (d) NRQD/nonhyperbolic. Darker regions indicate higher local density of states (LDS). The minimum and maximum LDS values of the patterns are $\left(2.59 \times 10^{-3}, 0.641\right),\left(5.84 \times 10^{-4}, 1.39\right),\left(8.50 \times 10^{-3}, 7.35 \times 10^{-2}\right)$, and $\left(1.40 \times 10^{-2}, 0.284\right)$ for (a-d), respectively. The color scale is normalized for each panel for better visualization. From Ref. [369] with permission.

transmission fluctuations with sharp resonances than the system with hyperbolic dynamics, which is consistent with results in the traditional study of quantum chaotic scattering. However, in GQDs, the fluctuations are much stronger with smaller energy scales as compared with NRQDs. The width of the transmission resonances provides the base of a theoretical explanation for the enhanced fluctuations in GQDs: scarring of quantum states in the graphene system is more pronounced, resulting in weaker coupling with the leads as compared with NRQDs. For example, it was found [369] that relativistic quasiparticles in graphene tend to stabilize themselves on the classically unstable periodic orbits. This can be demonstrated directly from the LDS patterns. Figure 40 shows a typical pattern for each of the four combinations. For nonhyperbolic NRQD and GQD, the LDS patterns are well localized, but the patterns for the GQD are much sharper than those for the NRQD. For the hyperbolic systems, the patterns associated with the NRQD are relatively weak, as exemplified by Fig. 40(c), but for the GQD, there are still many well pronounced pointer states, as the one shown in Fig. 40(a). In general, the transmission (or scattering-matrix elements) will exhibit characteristically enhanced fluctuations in relativistic than in nonrelativistic quantum mechanics.

\section{Quantum chaotic scattering for bilayer graphene quantum dots}

From Sec. V C, one sees that, in relativistic quantum dots made of monolayer graphene, systems with mixed classical dynamics exhibit sharper conductance fluctuations than those with chaotic classical dynamics, as for nonrelativistic quantum systems. However, even when the classical dynamics are fully chaotic, monolayer graphene quantum dots permit highly localized states, leading to Fano-like resonances with sharp conductance fluctuations. This suggests that quasiparticles in a chaotic graphene confinement can make the classically unstable orbits somewhat more "stable" in relativistic quantum systems, implying that the interplay between chaos and relativistic quantum mechanics can lead to phenomena that are not present in nonrelativistic quantum systems.

A unique feature of monolayer graphene is that the quasiparticles are massless Dirac fermions. 
The interplay between finite mass and chaos in relativistic quantum transport was then studied [379] with a focus on the generality of persistently sharp conductance fluctuations in relativistic quantum chaotic systems. In particular, chaotic bilayer graphene quantum dots (BGQD) were used [379] as a prototypical class of systems, in which the quasiparticles have a finite mass. It was found that persistently sharp conductance fluctuations are still present in BGQDs, indicating that a finite mass is not capable of breaking the localized states. It was also found that, in bilayer graphene quantum dots, electrons tend to "hop" between the two layers along the classical ballistic trajectory in each layer. As a result, the local density of states (LDS) for one layer does not form an "orbit" per se: an "orbit" emerges only when the LDS for both layers are combined. The results indicate that in both massless and massive chaotic relativistic quantum systems, Fano-like resonances and sharp conductance fluctuations are a common feature.

Model of chaotic bilayer graphene systems. Bilayer graphene is composed of two coupled monolayers of carbon atoms, each with a hexagonal lattice structure. The AB stacking bilayer graphene model [380] can be used, which includes inequivalent $A_{1}$ and $B_{1}$ atoms in the top layer and $A_{2}$ and $B_{2}$ atoms in the bottom. The two graphene layers are arranged in such a way that the $\mathrm{A}_{1}$ atoms are directly above the $\mathrm{B}_{2}$ atoms, while $\mathrm{B}_{1}$ or $\mathrm{A}_{2}$ atoms are above or below the center of hexagons in the other layer. The tight-binding Hamiltonian was used [379], which characterizes the electronic structure of graphene reasonably accurately [381] and is applicable to systems of a finite number of layers [105-107], is given by [380]

$$
H=-\gamma_{0} \sum_{l,<i, j>}\left(a_{l, i}^{\dagger} b_{l, j}+H . c .\right)-\gamma_{1} \sum_{i}\left(a_{1, i}^{\dagger} b_{2, i}+H . c .\right)-\gamma_{3} \sum_{<i, j>}\left(b_{1, i}^{\dagger} a_{2, j}+H . c .\right) \text {, }
$$

where $a_{l, i}^{\dagger}\left(b_{l, i}^{\dagger}\right)$ and $a_{l, i}\left(b_{l, i}\right)$ are the creation and annihilation operators for sublattice $\mathrm{A}(\mathrm{B})$ at site $R_{l, i}$ in layer $l(1,2), H . c$. denotes Hermitian conjugate, $\gamma_{0} \equiv t$ is the nearest-neighbor hopping energy in a single layer (hopping between different sublattices), $\gamma_{1}$ and $\gamma_{3}$ are energies for the hopping processes $\mathrm{A}_{1} \longleftrightarrow \mathrm{B}_{2}$ and $\mathrm{B}_{1} \longleftrightarrow \mathrm{A}_{2}$, respectively, which represent the interlayer coupling. The coupling parameters have the standard values [382]: $\gamma_{0}=2.8 \mathrm{eV}, \gamma_{1}=0.4 \mathrm{eV}$, and $\gamma_{3}=0.3 \mathrm{eV}$.

In the momentum space, the low energy bands can be approximated as $E \approx \sqrt{\gamma_{1}^{2} / 4+v^{2} p^{2}}-$ $\gamma_{1} / 2$, where $p$ and $v$ are the momentum and Fermi velocity, respectively [383]. A quasiparticle in the bilayer graphene can thus be regarded effectively as a massive relativistic fermion [383], as opposed to massless particles in monolayer graphene [105-107, 381].

Transmission fluctuations in BGQDs. The characteristics of quantum scattering dynamics in open BGQDs with distinct types of classical dynamics can be studied again by using the cosine billiard $[384,385]$ as the confinement domain of the quantum dot. The transmission fluctuations through the BGQDs that correspond to three distinct types of classical dynamics: mixed, intermediate, and chaotic were studied [379]. For comparison, results of NRQDs were also included. In the simulations, the maximum number of propagating modes was chosen to be $N_{\text {mode }}=96$ for all cases for BGQDs, and $N_{\text {mode }}=48$ for NRQDs, so their leads have comparable width. The boundaries for the BGQDs were assumed to be zigzag in the horizontal direction. Figure 41 shows the transmission coefficient $T$ of the three types of BGQDs versus the Fermi energy, together with the results from NRQDs with the same corresponding classical dynamics. It can be seen that, for both BGQDs and NRQDs, as the classical dynamics change from mixed to chaotic, the conductance fluctuations become progressively smooth. This is consistent with previous results of quantum chaotic scattering in monolayer graphene quantum dots (MGQDs) [369]. However, when com- 

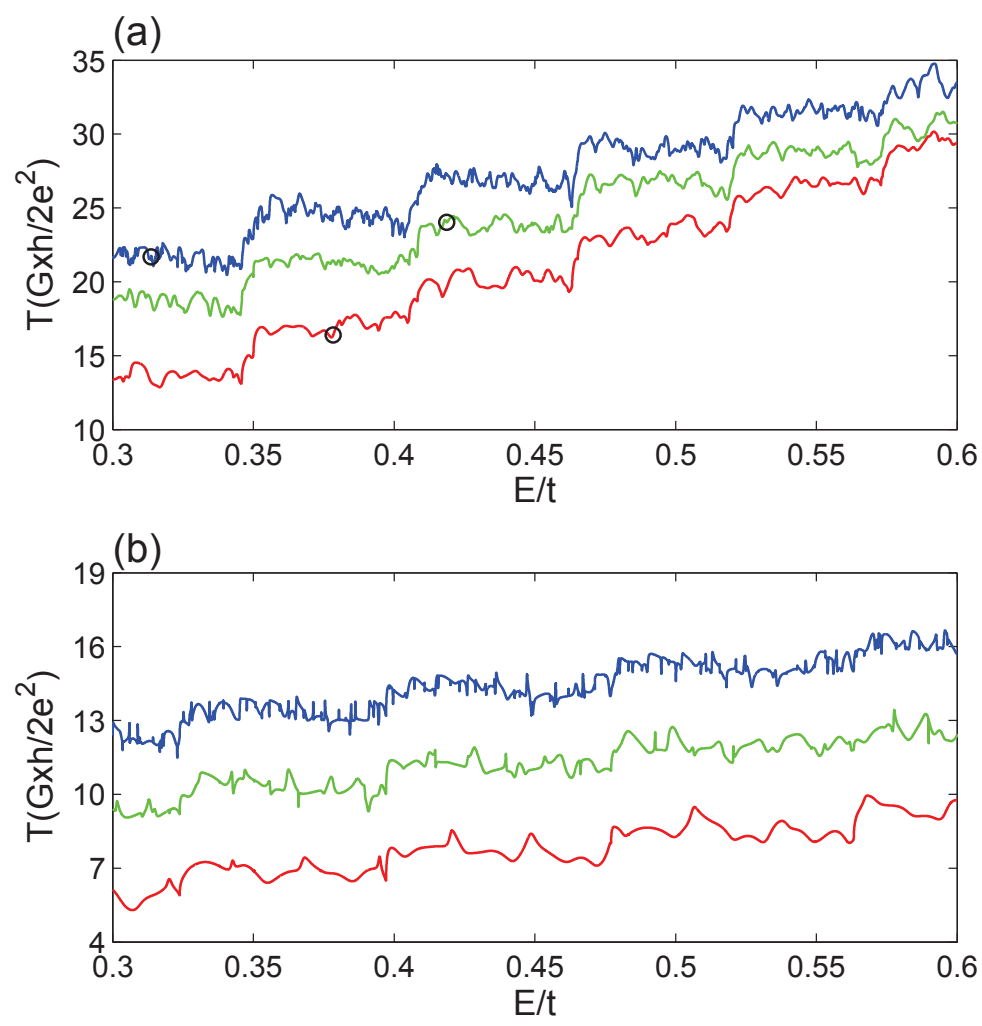

FIG. 41. Transmission fluctuations in BGQDs and NRQDs. Shown are the various transmission versus energy for the cosine billiard quantum dot in both relativistic and nonrelativistic regimes: (a) bilayer graphene (BGQD); (b) NRQD. The (blue/green/red) curves from top to bottom are for mixed/intermediate/chaotic cases: $W / L=0.36 \& M / L=0.22, W / L=0.27 \& M / L=0.165$, and $W / L=0.18 \& M / L=0.11$, respectively. The circles in (a) indicate the energy values used in Fig. 44. For all case, the number of transverse modes is $N_{\text {mode }}=96$ for BGQDs and $N_{\text {mode }}=48$ for NRQDs so that their leads have comparable width. From Ref. [379] with permission.

paring the BGQDs with the corresponding NRQDs, it can be seen that for the same geometry (especially in the chaotic case - the bottom curves in both panels), the conductance fluctuations for the latter are nearly perfectly smooth, while for BGQDs there are still sharp conductance fluctuations.

The effects of the wavefunction patterns on the values of $\gamma_{\alpha}$ in BGQDs were examined [379]. Figure 41 also shows that, as the classical dynamics becomes more chaotic, the conductance curves exhibit less sharp fluctuations. This reasoning suggests that $\gamma_{\alpha}$ for various resonant states should shift toward increasingly larger values as the classical dynamics of the system becomes progressively more chaotic. Results from a systematic calculation of the imaginary part $\gamma_{\alpha}$ for the various settings are shown in Fig. 42. The energy at which the self-energy matrix is evaluated is $E_{0}=0.2 t$ for BGQD [Fig. 42(a-c)] and $E_{0}=t$ for NRQD [Fig. 42(d-f)]. Since the calculated $E_{\alpha}$ values are accurate only in the vicinity of $E_{0}$, the energy intervals in Figs. 42(a-f) were chosen to be relatively small and close to $E_{0}$. Note that the upper curves of the $\gamma_{\alpha}$ values are in the range of $0.01 t$ to $0.1 t$, which correspond to the smooth variations in the conductance curve, and the smaller $\gamma_{\alpha}$ values correspond to the sharp conductance resonances. Figures 42(a) and 42(d) reveal that, for mixed 

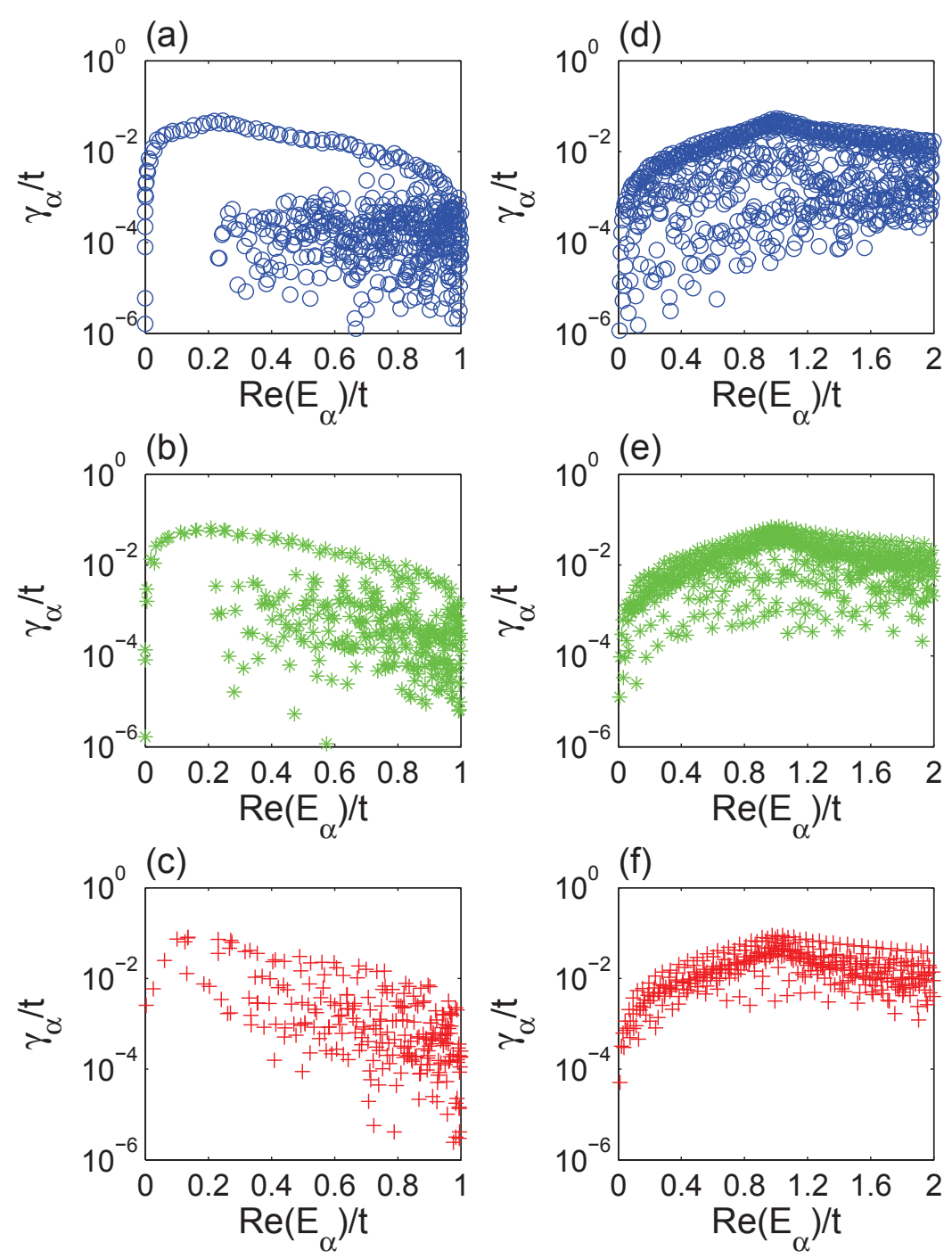

FIG. 42. Characteristics of Fano resonances in BGQDs. Shown are the resonance width $\gamma_{\alpha}$ for bilayer graphene quantum dots and their nonrelativistic quantum counterparts for different types of classical dynamics: (a) BGQD/mixed (b) BGQD/intermediate (c) BGQD/chaotic, (d) NRQD/mixed (e) NRQD/intermediate, and (f) NRQD/chaotic. The energy values at which the self-energy is evaluated are $E_{0}=0.2 t$ for (a-c) and $E_{0}=t$ for (d-f). From Ref. [379] with permission.

classical dynamics, both BGQD and NRQD have $\gamma_{\alpha}$ values as small as $10^{-6} t$, leading to the sharp conductance fluctuations in the top curves of Fig. 41. As the classical dynamics become more chaotic, the values of $\gamma_{\alpha}$ shift upwards, as shown in Figs. 42(b) and 42(e). For the fully chaotic systems [Figs. 42(c) and 42(f)], the values of $\gamma_{\alpha}$ are large and their spread is small. Figure 43 shows the averaged value of $\log _{10}\left(\gamma_{\alpha} / t\right)$ in a moving window to remove the fluctuations presented in Fig. 42. Since the upper curves of the $\gamma_{\alpha}$ values correspond to the smooth conductance variations, it is excluded from the average. In general, classical chaos can affect the conductance fluctuations for both nonrelativistic and relativistic quantum dot systems (for the latter regardless of zero or finite mass). For relativistic quantum systems, however, the effect of chaos-induced enlargement of $\gamma$ is less dramatic as compared with the nonrelativistic case. 

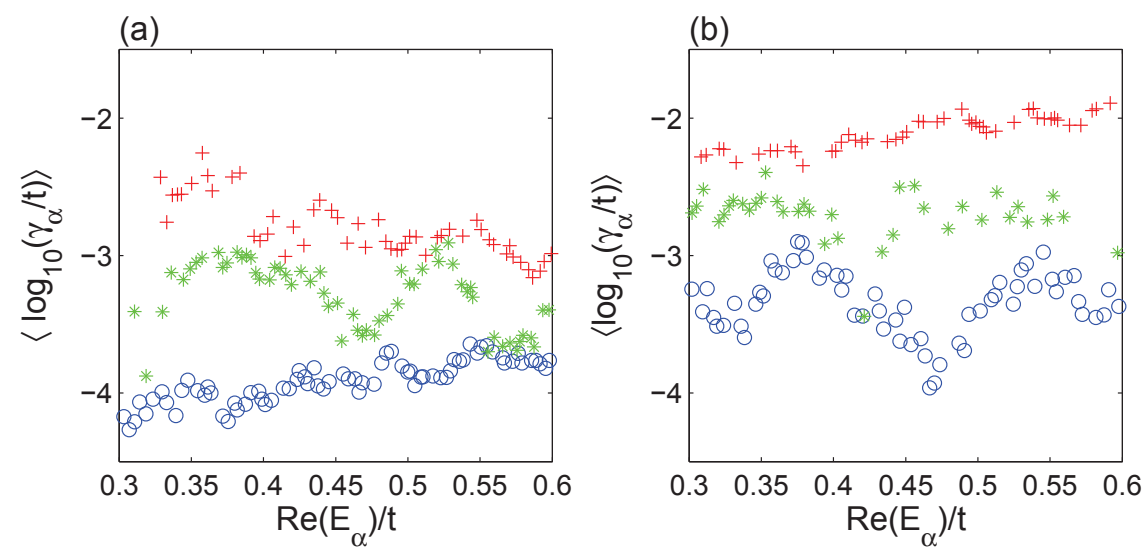

FIG. 43. Detailed behaviors of Fano resonance width for systems with different types of classical dynamics. Shown are the the mean values of $\log _{10}\left(\gamma_{\alpha} / t\right)$ in a moving window with window size $0.04 t$ and step size 0.004t. The upper curves in different panels in Fig. 42 are excluded in the average as they account for the smooth variation rather than the sharp conductance resonances. Panel (a) is for BGQD (left panels in Fig. 42) and panel (b) is for NRQD (right panels in Fig. 42). The blue circles, green asterisks, and red plus symbols are for mixed, intermediate, and chaotic dynamics in the classical limit, respectively. From Ref. [379] with permission.

Localized LDS and Electron flow patterns. Qualitative insights into the role of classical dynamics in the conductance fluctuation patterns in BGQDS can be gained by calculating the LDS and electron flow patterns for some representative energy values. For a bilayer graphene system, the interlayer hopping energy is relatively weak as compared to the intralayer counterpart, i.e., the values of $\gamma_{1}$ and $\gamma_{3}$ are about one tenth of $\gamma_{0}$, and the LDS and electron flow patterns are not identical for the two layers. For simplicity, one can focus on the $x$-component of the electron flow. In Fig. 44, the left and middle columns show the LDS and electron flow patterns for each layer, and the right column shows the combined patterns from both layers. It can be seen that the LDS and electron flows are highly correlated, e.g., they are localized in the same region of the dot. The $y$ - and $z$-components of the current show similar features. The two layers are strongly coupled so that the electrons flow back and forth between the two layers, as can be identified unambiguously in Fig. 44(a), where in certain regions, the electrons and their flow are mostly localized to one layer but to the corresponding adjacent regions on the other layer. When the patterns from both layers are combined, the LDS patterns reveal the underlying classical periodic orbits. A comparison of the LDS and electron flow patterns for different cases, as illustrated in Fig. 44, indicates that classically mixed dynamics can lead to relatively more pronounced localized states, but the localized patterns weakened as the classical phase space contains more chaotic regions. The degree of localization can be qualitatively described by the ratio of the maximum to the minimum values of LDS, where a larger ratio corresponds to stronger localization, as shown in Fig. 45. The typical values of the ratio for BGQDs are about $10^{3}$, a few hundreds, and less than one hundred for mixed, intermediate, and chaotic cases, respectively. The values for the NRQDs are comparably smaller. The electron flow pattern show similar features. A comparison between the patterns for BGQDs and NRQDs indicates that the former exhibits more localized LDS and thus sharp conductance fluctuations. This is consistent with previous results on monolayer graphene systems in the context of transport [369], tunneling [135, 181], and spectral statistics [282]. 

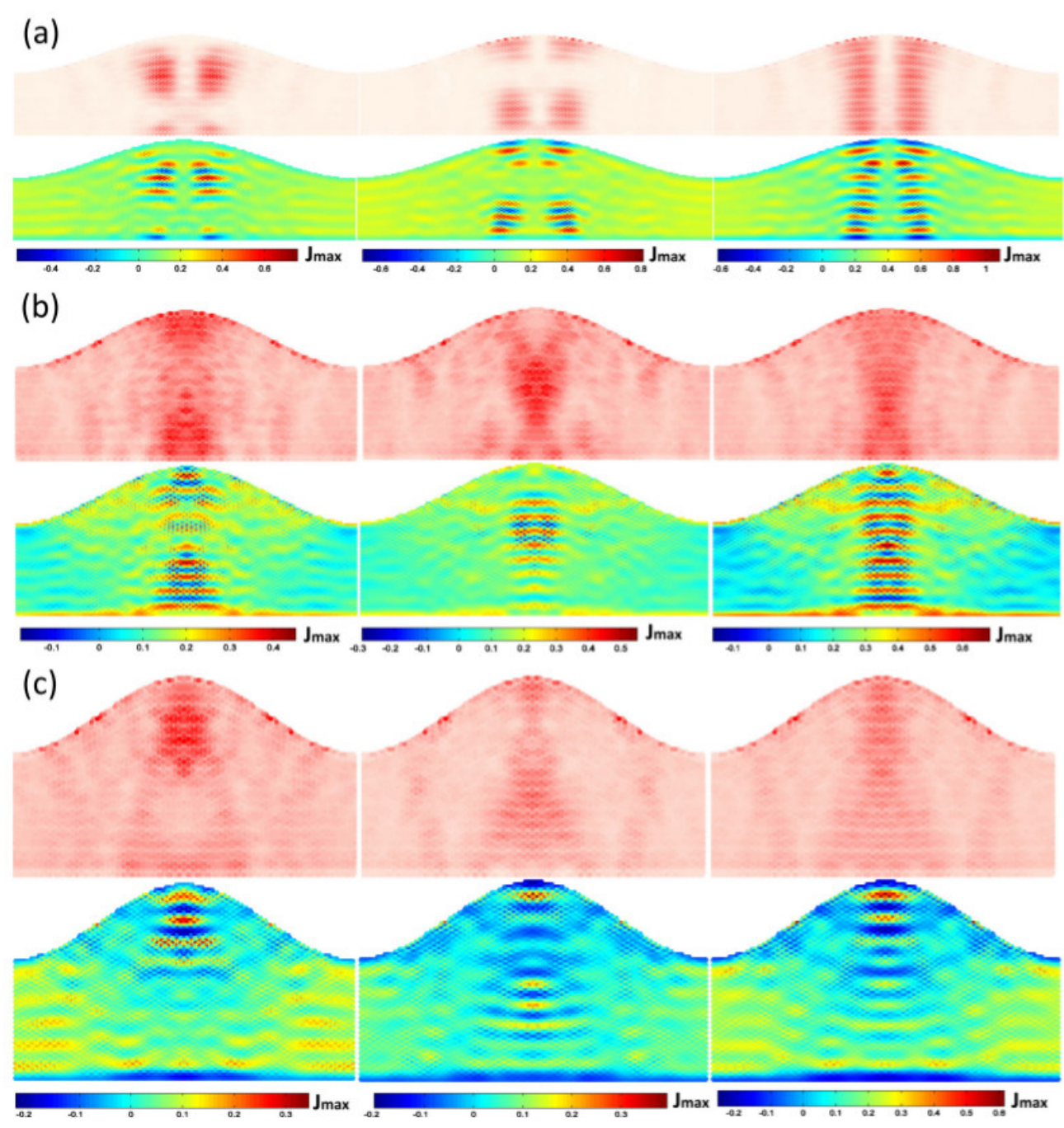

FIG. 44. Understanding resonances through wavefunction patterns in BGQDs. Representative local density of states (LDS, the first row) and the corresponding $x$-component of the electron flow (the second row) for BGQDs with mixed (a), intermediate (b), and chaotic (c) classical dynamics. For the LDS patterns, red region means higher values, and the color scale is normalized for each panel for better visualization. Left, middle, and right panels are for the first layer, the second layer, and combined layers. The Fermi energies are $0.3137 t$ for (a), $0.4187 t$ for (b), and $0.3784 t$ for (c), with the respective maximum and minimum values of the LDS patterns being $\left(3.0016,1.2 \times 10^{-3}\right),\left(0.4987,7.7 \times 10^{-3}\right)$, and $\left(0.4823,5.6 \times 10^{-3}\right)$. From Ref. [379] with permission.

\section{E. Quantum chaotic scattering in graphene systems in the absence of invariant classical dynam-} ics

A tacit assumption employed in many works on quantum chaotic scattering is that, as a physical parameter such as the particle energy changes, the classical dynamics is invariant so that the decay law remains unchanged, rendering meaningful its Fourier transform that is directly related to the energy correlation function of the scattering matrix elements [173]. Such situations arise in electronic transport in the absence of any magnetic field where the corresponding classical dynamics 

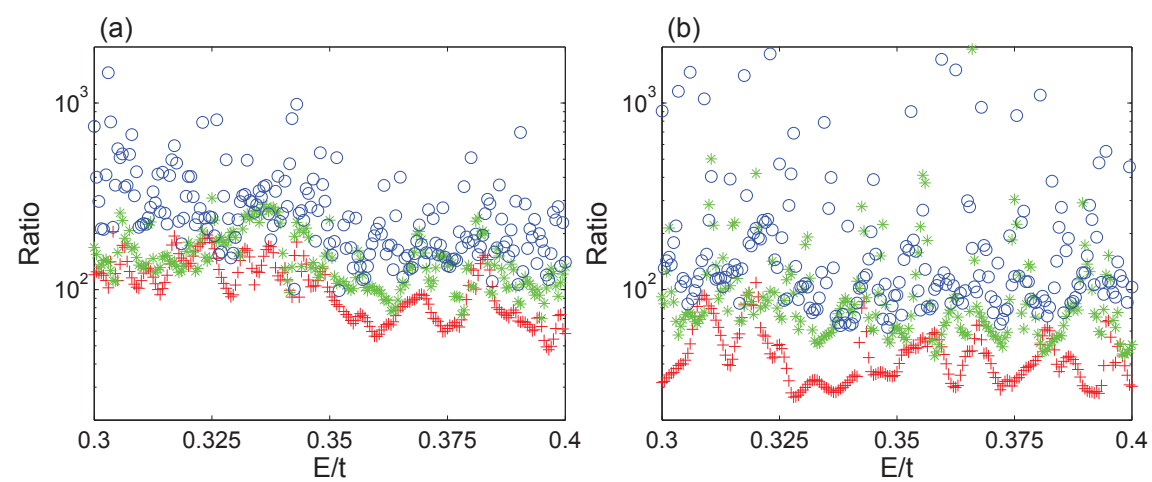

FIG. 45. Characterization of the degree of localization of quantum states for BGQDs. Shown are the values of the ratio of the maximum to the minimum values of the LDS versus energy: (a) BGQD and (b) NRQD. From top to bottom the blue circles, green asterisks, and red plus symbols are for mixed, intermediate, and chaotic cases, respectively. From Ref. [379] with permission.

are essentially that of a open billiard and do not depend on the electron energy. However, when a magnetic field is present, the characteristics of classical dynamics can change drastically with the particle energy. For example, in an early work, Breymann, Kovács and Tél studied chaotic scattering of charged particles in an open three-disk billiard subject to a perpendicular magnetic field [386]. Classically, since the Lorentz force depends on both the particle energy and the magnetic field, even for a fixed magnetic field strength, the dynamics will depend on the energy. Considering the quantum counterpart, a challenge is then that, as the particle energy is systematically changed, there is no unique classical correspondence. Quantum mechanically, the scattering matrix elements will exhibit fluctuations with the particle energy, but the Fourier-transform formula relating the energy autocorrelation function and classical particle decay law is no longer applicable. How can then the fluctuation patterns be characterized and understood?

The absence of an invariant set for chaotic scattering dynamics can be illustrated [130] using a symmetric, three-terminal quantum-dot system, as shown in the left of Fig. 46. Classically, the system consists of a scattering region and three semi-infinite leads separated angularly by $2 \pi / 3$. The scattering region is specified by the black heavy lines, the boundaries of which consist of three circular arcs, where the centers of the respective circles are located at the three vertices of an equilateral triangle, the coordinates of which are $(-d / \sqrt{3}, d),(-d / \sqrt{3},-d),(2 d / \sqrt{3}, 0)$, respectively. The radius of each circle is $r$. Suppose the quantum-dot system is made entirely of graphene. The geometric parameters $d$ and $r$ can then be chosen in terms of the lattice constant of graphene: $d=199 a_{0}$ and $r=110 \sqrt{3} a_{0}$. The boundary walls are infinitely hard, rendering elastic scattering off the walls. The semi-infinite leads are also assumed to be made of graphene. A perpendicular magnetic field is uniformly applied in the circular region enclosed by the blue-dashed lines in Fig. 46, the radius of which is $d / \sqrt{3}$. Due to the dependence of the Lorentz force on particle velocity and hence on particle energy, the classical dynamics depends on the energy as well. This means that, for the quantum-dot system, as the Fermi energy is varied, the corresponding classical phase-space structure will change accordingly [386]. In classical simulations, the electron moves effectively in free space, so its energy is given by $E=m v^{2} / 2$ and the cyclotron radius is $r=m v / e B$, where $m$ is the vacuum mass of the electron and $v$ is the Fermi velocity.

The right half of Fig. 46 shows, for a fixed magnetic field specified by the magnetic flux $\Phi / \Phi_{0}$, some representative chaotic sets for different values of the Fermi energy. For convenience of 

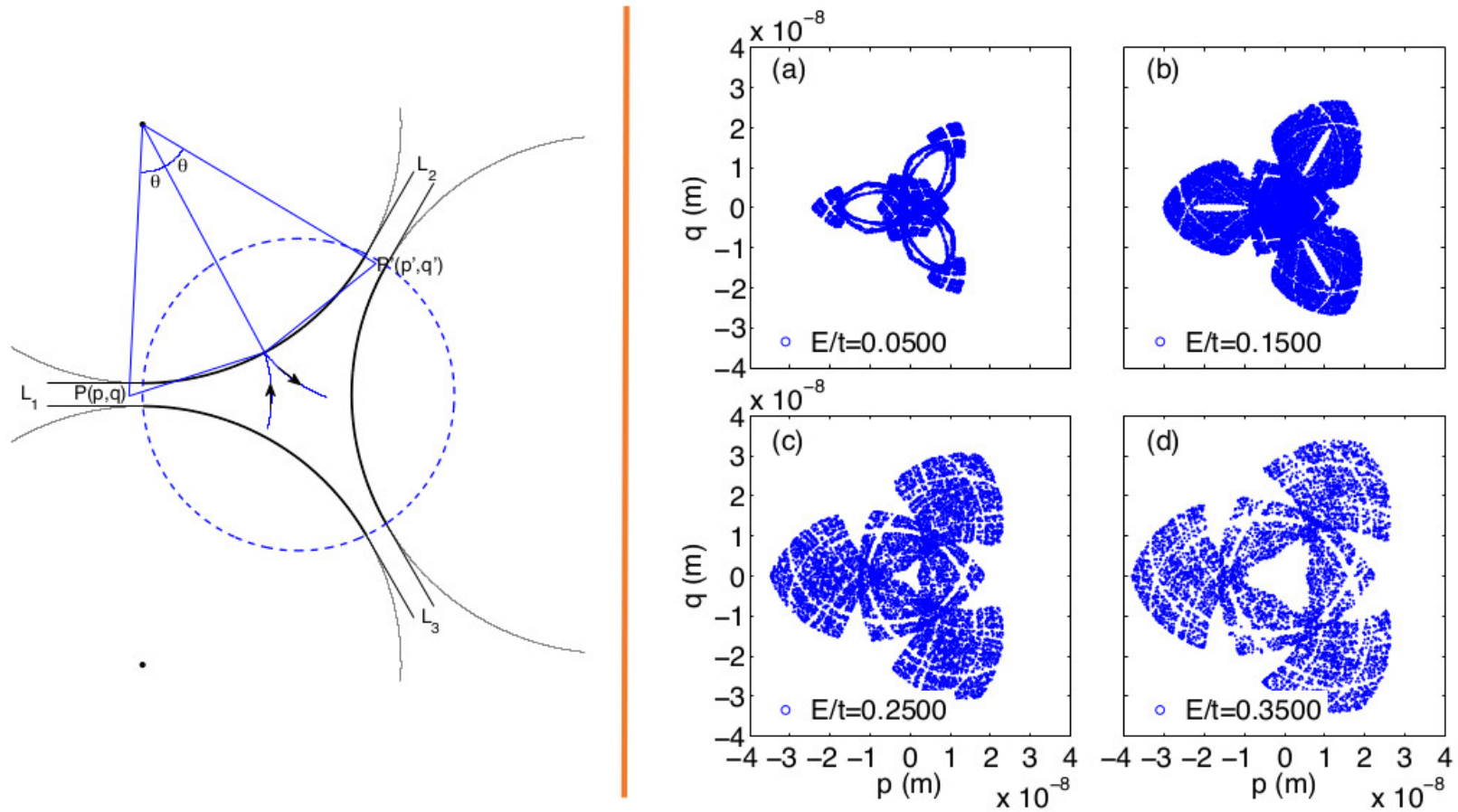

FIG. 46. An illustrative three-terminal classical chaotic scattering system in the presence of a magnetic field, whose dynamical invariant set depends on the electron Fermi energy. Left half: geometry of a three-terminal quantum dot system. The boundary of the device is indicated by the black heavy lines. A perpendicular magnetic field is applied in the circular region defined by the blue dashed lines. The system is made entirely of graphene. The corresponding classical dynamics is effectively that of an open billiard system with three outgoing channels. Right half: for a fixed magnetic field specified by $\Phi / \Phi_{0}=0.0019$, representative chaotic sets for four different values of the Fermi energy: (a) $E / t=0.05$, (b) $E / t=0.15$, (c) $E / t=0.25$, and (d) $E / t=0.35$, where $p$ and $q$ are the coordinates of center points of the electron trajectory. It can be seen that the chaotic sets are characteristically distinct.

quantum calculations, the magnetic flux quanta can be used to normalize the magnetic flux. For graphene, the magnetic flux is $\Phi=B S$, where $S=3 \sqrt{3} a_{0}^{2} / 2$ is the area of one honeycomb unit cell composed of six carbon atoms. The electron Fermi energy can also be normalized by the hopping energy in the tight-binding model of graphene lattice. For a fixed magnetic-field strength, as the Fermi energy of the conducting electrons increases, the underlying chaotic scattering dynamics in the classical limit can change its characteristics, and the quantum conductance can exhibit significant fluctuations.

Examining the poles of the S-matrix associated with the non-Hermitian, effective Hamiltonian of the interior system is the standard way to study quantum chaotic-scattering systems [387-391], which is effectively a random-matrix based approach. In the presence of a magnetic field where time-reversal symmetry is broken, the non-Hermitian Hamiltonian can be regarded as being drawn from GUE. The effective random-matrix type of Hamiltonian can be written as $\boldsymbol{H}_{\mathrm{eff}}=\boldsymbol{H}-i \boldsymbol{\Gamma}$, where $\boldsymbol{H}$ is the Hermitian matrix describing the scattering region and $\boldsymbol{\Gamma}$ describes the transition from the scattering region to the leads. The complex eigenvalues of $\boldsymbol{H}_{\text {eff }}$ can then be used to characterize the poles of the S-matrix [387-391]. By performing the non-perturbative ensemble averaging, one can obtain the statistical properties of the S-matrix, e.g., distribution of resonance width, 
phase shift, and delay time, etc. For example, in the regime of wide, overlapping resonances, the Smatrix elements behave smoothly, while near a sharp resonance the S-matrix elements will change abruptly. Because of the universality of the random matrices in describing the quantum dynamics of classically chaotic systems, close or open, the random-matrix based theory of quantum chaotic scattering is general and can reveal universal statistical properties of the underlying system. In Ref. [130], the matrix $\Gamma$ is nothing but the self-energies that quantify the effects of the open leads on the otherwise closed, Hermitian system through the non-equilibrium Green's functions, and such self-energy terms can be calculated explicitly when the scattering system is specified. The approach is thus capable of yielding a concrete form of the non-Hermitian Hamiltonian with respect to any specific configuration of the scattering system, which offers tremendous advantages in experimental design and actual device development based on quantum chaotic-scattering dynamics. For chaotic graphene quantum dot systems under a perpendicular magnetic field, this approach was indeed effective for understanding the fluctuation properties associated with quantum chaotic scattering even in the absence of invariant classical phase-space structure [130].

What about conductance fluctuations when the strength of the magnetic field is allowed to vary? While in general, the fluctuations depend on the nature of the corresponding classical dynamics, i.e., integrable or chaotic, in graphene quantum-dot systems, when a strong magnetic field is present, signatures of classical dynamics can disappear and universal scaling behaviors emerge. For example, a systematic study [147] of transport through various integrable and chaotic graphene quantum dot systems revealed that, in the parameter plane spanned by the perpendicular magnetic flux and the Fermi energy, there are regions of regular and random conductance oscillations, respectively. As the Fermi energy or the magnetic flux is changed, the fluctuations can be either regular or random, implying a kind of "coexistence" of regular and irregular conductance fluctuations as a single physical parameter is varied. This has implications to experiments, where a significant issue is how conductance fluctuations are affected by the size of the quantum dot in the presence of a perpendicular magnetic field. For example, in the experimental study [392] of quantum dots of size ranging from $0.7 \mu \mathrm{m}$ to $1.2 \mu \mathrm{m}$, the authors found nearly periodic conductance oscillations as the magnetic-field strength is varied. The frequency of the oscillation pattern, the so-called magnetic frequency, was found to follow a scaling relation with the edge size of the dot [392]. In another study of the magnetic scaling behavior in graphene quantum dots [159, 393], it was found that for small dots of edge size less than $0.3 \mu \mathrm{m}$, the magnetic frequency exhibits a scaling relation with the dot area. Focusing on a dominant set of scarred orbits and examining the resulting conductance oscillations revealed [147] that, for graphene quantum dots, below the first Landau level, the conductance exhibits periodic oscillations with the magnetic flux and with the Fermi energy. In fact, the magnetic frequency scales linearly with the dot size. However, the energy frequency, the inverse of the variation in the Fermi energy for the conductance to complete one cycle of oscillation, scales inversely with the dot size. Beyond the regime of periodic conductance oscillations, new sets of scarred orbits emerge and evolve as successive Landau levels are crossed, each with its own period, leading to random conductance fluctuations. The remarkable feature is that these scaling behaviors are independent of the nature of the underlying classical dynamics, i.e., regular or chaotic. 


\section{F. Conductance stability in chaotic and integrable graphene quantum dots doped with random impurities}

In the development of nanoscale quantum devices, an important issue is stability against random perturbations such as various types of impurities. While the impurities can be reduced to certain extent through the improvement and refinement of the underlying fabrication process, it is of interest to uncover alternative mechanisms to enhance the device stability. This subsection shows that classical chaos can be exploited to generate devices that are relatively more stable in the quantum regime than those exhibiting integrable dynamics in the classical limit.

The issue of conductance stability in graphene quantum dots was studied in Ref. [394], where the effects of an ensemble of random impurities of systematically varying strength on some appropriately averaged value of the conductance were investigated. It is convenient to focus on the average conductance over the corresponding single-mode Fermi-energy range. This energy range is classically small but quantum mechanically large, rendering applicable semiclassical treatment of the scattering dynamics [172]. To contrast the role of classical dynamics, two types of geometric domains were chosen for the dot region: stadium and rectangle, which generate classical chaotic and integrable dynamics, respectively. As the strength of the random impurities is increased from zero, the average conductance will decrease due to localization of wavefunctions. However, it was found [394] that the integrable dot system exhibits a much faster decrease in the average conductance than that for the chaotic dot system, implying a stronger conductance stability for the latter.

Figures 47(a) and 47(b) show, for classically chaotic and integrable geometries, respectively, typical behaviors of the ensemble-average conductance versus the Fermi energy and the impurity strength. The corresponding contour plots are shown in Figs. 47(c) and 47(d). For fixed energy values, two types of behaviors arise in the variation of the conductance with the impurity strength: (1) the conductance increases first as the impurities become stronger, reaches a maximum, and then decreases, and (2) the conductance decreases monotonically with the impurity strength. The first case is somewhat counterintuitive, as exemplified in Fig. 47(e) for $E / t \approx 0.115,0.233$ for the chaotic dot. This "stochastic" resonance-like phenomenon had been reported [146], where the initial conductance enhancement can be attributed to the breakdown of the edge states in graphene by weak impurities. However, for strong impurity the quantum states are localized, reducing the conductance. When such a resonance phenomenon occurs, the conductance value for the zero impurity case must be close to zero. That is, when the system is free of any random impurity, the system is already in some localized state, providing a "room" for impurity to break the state and consequently to enhance the conductance. Two examples of the localized states in the absence of any impurity are shown in Figs. 47(g) and 47(h), respectively, for the chaotic and integrable dots. If, for certain Fermi energy, in the absence of any impurity the quantum state is not localized so that the conductance has a relatively large value, introducing impurities into the system can only serve to reduce the conductance, ruling out any possible increase in the conductance and consequently the "stochastic" resonance phenomenon.

To address the conductance stability and to better understand the effect of the interplay between random impurities and classical dynamics on conductance, the average conductance was calculated [394] as a function of the impurity strength. Concretely, for a fixed value of the impurity strength, the conductance over the Fermi energy in the range defined by the first transmission mode of the graphene dot was averaged, which is about 0.24t. For the dot parameters as in Fig. 47, 

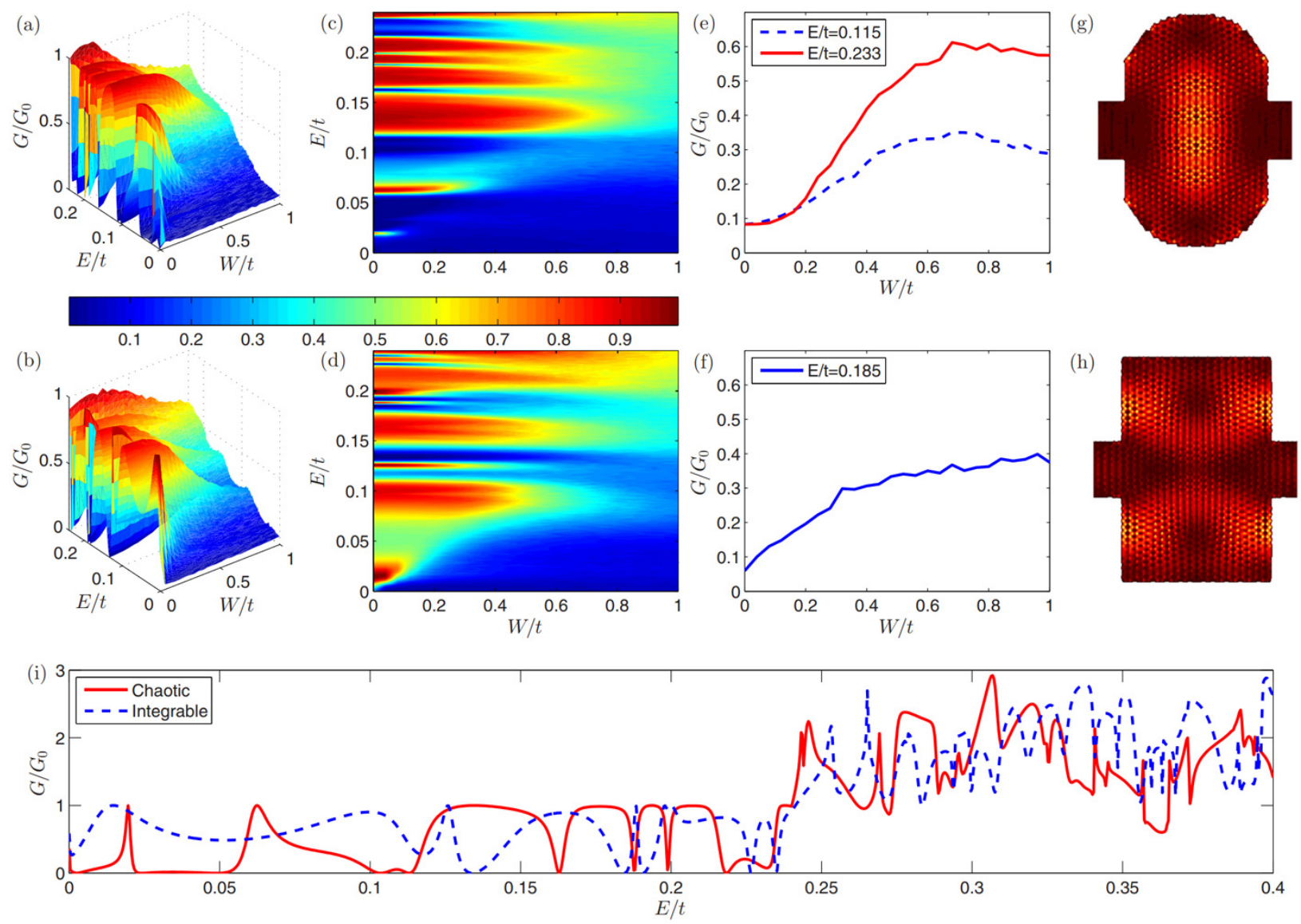

FIG. 47. Dependence of the average conductance on the Fermi energy and impurity strength. (a,b) Three-dimensional plot of the conductance versus the Fermi energy and the impurity strength for classically chaotic and integrable dots, respectively. (c,d) The corresponding contour plots. (e) Two cases of resonancelike phenomenon for the chaotic geometry for $E / t \approx 0.115$ (red solid line), 0.233 (blue dashed line). (f) Conductance resonance for the integrable geometry for $E / t \approx 0.185$. (g,h) Examples of localized states in absence of any random impurity for the chaotic and integrable cases, respectively. The device structural parameters are: lead width $w \approx 36.92 \AA$, dot width $d \approx 78.7 \AA$, and dot length $l \approx 151.94 \AA$. (i) Conductances versus the Fermi energy of the chaotic (red solid line) and integrable (blue dashed line) devices without impurities. For both devices, the width of the first transmission mode is about $0.24 t$. The conductance unit is $G_{0}=2 e^{2} / h$. From Ref. [394] with permission.

the normalized average conductance is shown in Fig. 48(a), where the blue and red curves correspond to the integrable and chaotic cases, respectively. One can see that, as the impurity strength is increased, the average (or overall) conductance decreases monotonically for both cases. However, for the chaotic dot, the slope of the decreasing trend is smaller than that for the integrable dot. This behavior persists with respect to variations in the device parameters. For example, Fig. 48(b) shows a case with the lead width reduced to $w \approx 15.62 \AA$. These results indicate that the conductance of the chaotic dot is more "stable" with respect to variations in the strength of random impurities.

A semiclassical theory was developed [394] to qualitatively explain this phenomenon and an understanding was obtained based on the random matrix theory through analyzing the local den- 

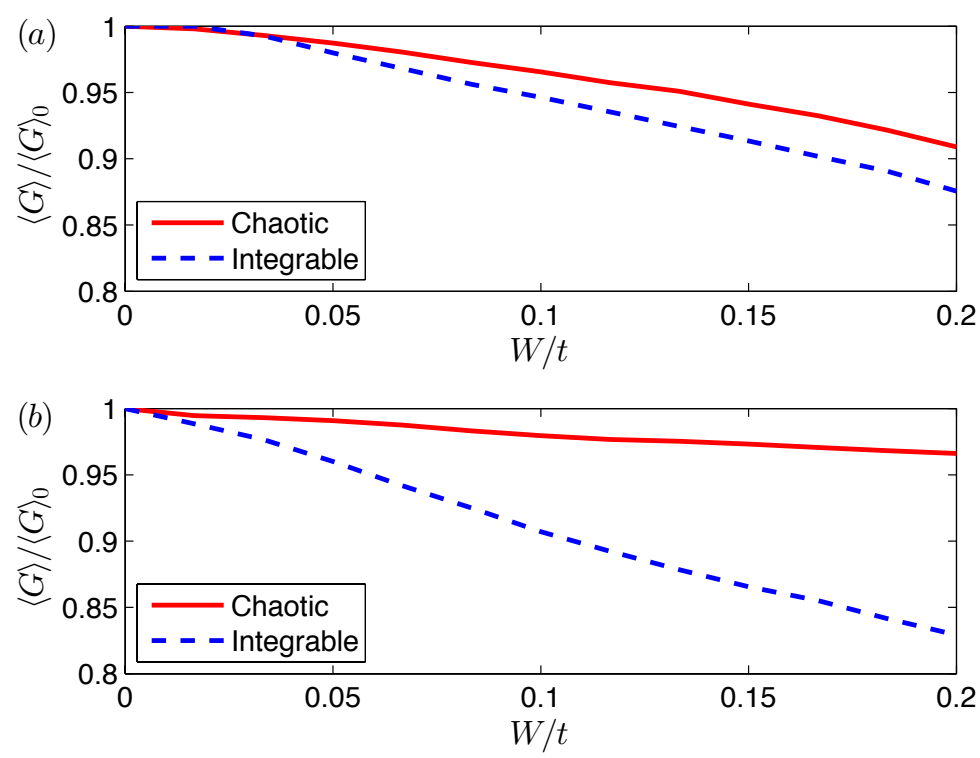

FIG. 48. Effect of lead width on conductance stability. Average conductance over the energy range of the first transmission mode of the lead versus the impurity strength for (a) lead width $w \approx 36.92 \AA$ and (b) lead width $w \approx 15.62 \AA$. The blue (dashed) and red (solid) curves correspond to classically integrable and chaotic dot structures, respectively. The quantity $\langle G\rangle_{0}=\langle G\rangle(W=0)$ was used to normalize the average conductance. Note that $\langle G\rangle_{0}$ should be distinguished from the natural conductance unit $G_{0}$. From Ref. [394] with permission.

sity of states in the dot region and the energy level statistics in the corresponding closed system. These findings strongly advocate the use of chaotic geometry in quantum dot structures, which is consistent with previous results in nonrelativistic quantum chaos on smooth conductance fluctuations in classically chaotic systems. Classical chaos has the benefit of bringing in greater stability to both nonrelativistic and relativistic quantum devices.

\section{G. Chaotic scattering in Dirac fermion systems}

Electron dynamics in graphene can be treated as relativistically quantum only approximately, as they are not exactly governed by the Dirac equation due to the existence of the non-equivalent valleys. This is especially the case for etched graphene quantum dot or confined geometries with abrupt edge terminations, in which inter-valley scattering can be substantial. Chaotic scattering in open graphene systems studied based on the discrete-lattice calculations is thus relativistic quantum only in an approximate sense. Therefore, to study "real" relativistic quantum chaotic scattering based on the continuous Dirac equation is necessary for uncovering generic phenomena. Moreover, results on the scattering problem obtained through the Dirac equations can explain some of the transport properties in 2D Dirac material systems, providing a deeper understanding of the associated unusual physics.

In the relativistic quantum realm, a unique scattering process can arise from the Klein paradox in the presence of an electrostatic potential barrier, with which particles can penetrate through an arbitrary height barrier in a non-evanescent way. With the peculiar linear dispersion relation 
for massless Dirac fermions, a strong incident-angle-resolved scattering behavior can arise from the barrier interface, where backscattering is generically absent at normal incidence while there is a sizable reflection probability upon increasing the incident angle. Classically, distinct types of scattering trajectories can be defined in terms of the nature of the underlying classical dynamics: integrable or chaotic. For example, an electrostatic potential defined two-dimensional scatterer of circular shape hosts regular trajectories resulting from continuous boundary scattering with a fixed angle of incidence, while the one of the stadium shape sustaining chaotic dynamics eventually will allow the particle to meet the boundary interface at normal incidence. Intuitively, one might expect unconventional quantum manifestations of classically integrable and irregular dynamics as a result of Klein tunneling based scattering.

There have been efforts to address the problem of relativistic quantum chaotic scattering in the presence of Klein tunneling [140, 142, 395, 396], where the Dirac equation for classically integrable and chaotic scatterer geometries is solved with an emphasis on the electrostatic confinement issue of massless Dirac fermions. In particular, based on the transfer-matrix scheme for a prototypical two-terminal setup with an electrostatic gate defined scatterer of a particular dot geometry embedded between two leads [140], Brouwer and collaborators demonstrated that perfect electrostatic confinement can be achieved if the classical dynamics are integrable and the quantum dynamics are separable, but classically chaotic dynamics can destroy confinement. Subsequently, a more powerful matrix Green's function method was developed to solve the scattering problem [395], where resonant scattering was studied and the unexpected behavior of persistent scattering resonances were uncovered in the limit of weak coupling regime for classically chaotic dynamics. This phenomenon contradicts the classical intuition. The main difference associated with classically integrable and chaotic dynamics lies in the scaling of the resonance width as a function of the coupling to the leads. For example, for the chaotic stadium shaped system, the width is proportional to the coupling for all resonances. However, for the integrable disk scatterer, the width varies as $(2 l+1)$ 'th power of the coupling, where $l>0$ denotes the well-defined angular momentum channel. In a follow-up study [142], it was argued that the difference is caused by the underlying nontrivial $\pi$ Berry phase leading to the non-vanishing half-integer values of the quantized total angular momenta, which excludes zero angular momentum states associated with normal incidence at the boundary. The argument was supported through the compensation of the Berry phase with the tunable Aharonov-Bohm phase arising from an applied magnetic flux. For the particular case where the two phases precisely cancel each other, the unexpected resonance behavior totally disappears for the chaotic stadium dot geometry, which is expected from a classical point of view. A subsequent study based on the density of states (instead of the two-terminal conductance) provided consistent results [396].

For massless Dirac fermions, in addition to scattering in the presence of Klein tunneling from a scalar type of potential, unconventional and potentially interesting scattering processes can arise when the system is subject to physically meaningful perturbations that have no counterparts in nonrelativistic quantum systems. For example, in systems that exhibit the superconducting-proximity effect, the resulting Dirac-Bogoliubov-de Gennes Hamiltonian allows one to define topologically nontrivial scatterers respecting both particle-hole and chiral symmetries. It was found that the preserved chiral symmetry can have a nontrivial effect on chaotic scattering, which is characterized by a significant dependence of the delay time or density of state distributions on the number of the emergent topological modes [397]. In $(2+1)$ dimensions, either a massive type or a magnetic skymion-type of scatterer can cause skew scattering of massless Dirac fermions, which is a typi- 
cal extrinsic mechanism for the anomalous Hall effect. Such behaviors are completely absent in the Schrödinger electron systems. At the present, the interplay between such exotic physics and classically nonintegrable dynamics is unknown.

Quite recently, a remarkable quantum scattering phenomenon in two-dimensional Dirac material systems was uncovered [398] where the manifestations of both classically integrable and chaotic dynamics emerge simultaneously and are electrically controllable. This is due to the breaking of the spin degeneracy through the Zeeman effect, leading to a vertical split of the Dirac cone. The distinct relativistic quantum fingerprints associated with different electron spin states were found to be due to a physical mechanism analogous to chiroptical effect in the presence of degeneracy breaking in the new area of Dirac electron optics [399-426]. The phenomenon mimics a chimera state in classical complex dynamical systems [427-434], but here in a relativistic quantum setting - henceforth the term "Dirac quantum chimera," associated with which are physical phenomena with potentially significant applications such as enhancement of spin polarization, unusual coexisting quasibound states for distinct spin configurations, and spin selective caustics. It was argued [398] that experimental observations of these phenomena are possible through, e.g., optical realizations of ballistic Dirac fermion systems. 


\section{ROLE OF CHAOS IN RELATIVISTIC QUANTUM RESONANT TUNNELING}

\section{A. Relativistic quantum tunneling - an introduction}

The role of classical chaos in quantum tunneling has been a topic of continuous interest in quantum chaos [174-181]. The remarkable phenomenon of chaos-regularized quantum tunneling was uncovered in 2011 [180], where it was found that classical chaos can significantly suppress the spread in the tunneling rate commonly seen in systems whose classical dynamics are regular. For example, consider a 2D system consisting of two symmetrical cavities connected by a 1D potential barrier along the line of symmetry. When the classical dynamics in each cavity are integrable, for sufficiently large energy the tunneling rate can assume many values in a wide interval. Choosing the geometry of the cavity such that the classical dynamics become chaotic can greatly enhance and regularize quantum tunneling.

Heuristically, the phenomenon of regularization of quantum tunneling by chaos can be understood, as follows. When the potential barrier is infinite, each cavity is a closed system with an infinite set of eigenenergies and eigenstates. Many eigenstates are concentrated on classical periodic orbits. For a classically integrable cavity, when the potential barrier becomes finite so that each cavity system is effectively an open quantum system, some stable or marginally stable periodic orbits continue to exist. However, because of the classically integrable dynamics, many surviving eigenstates correspond to classical periodic orbits whose trajectories do not encounter the potential barrier, generating extremely low tunneling rate even when the energy is comparable with or larger than the height of the potential barrier. The eigenstates corresponding to classical orbits that interact with the potential barrier, however, correspond to relatively strong tunneling. In a small energy interval, the quantum tunneling rate can thus spread over a wide range. However, when the classical dynamics are chaotic, isolated orbits that do not interact with the potential barrier are far less likely and, consequently, the states associated with low tunneling rates disappear, effectively suppressing the spread in the tunneling rate.

In this Section, three issues will be discussed: (1) relativistic quantum tunneling in fully developed chaotic systems, (2) role of mixed (nonhyperbolic) classical dynamics in relativistic quantum tunneling, and (3) resonant tunneling in graphene systems in the presence of chaos and electronelectron interactions.

Relativistic quantum tunneling in fully developed chaotic systems. The question of whether chaos can regularize tunneling in relativistic quantum systems was addressed in Refs. [134, 135] for both Dirac fermion and graphene systems, where the existence of surviving eigenstates leading to extremely low tunneling rates was confirmed when the classical dynamics are integrable. Similar to nonrelativistic quantum tunneling, making the cavities classically chaotic can greatly regularize the quantum tunneling dynamics. A unique feature that finds no counterpart in nonrelativistic quantum tunneling systems is the high tunneling rate in the regime where the particle energy approaches zero. This is a manifestation of the Klein-tunneling phenomenon [154, 265, 266]. It was demonstrated $[134,135]$ that the concepts of self-energies and the complex energy spectrum of the non-Hermitian Hamiltonian for the "open" cavity can lead to a qualitative understanding of regularization of tunneling in chaotic relativistic quantum systems.

Role of mixed (nonhyperbolic) classical dynamics in relativistic quantum tunneling. Nonhyperbolicity, as characterized by the coexistence of KAM tori and chaos in the phase space, is generic in classical Hamiltonian systems. A convenient system is the mushroom billiard, whose 
classical dynamics were mathematically proven to be nonhyperbolic [435]. A previous study of tunneling of Dirac fermion in the mushroom billiard system revealed [135] that the tunneling rate as a function of the energy exhibits a striking "clustering" phenomenon, where majority of the values of the rate concentrate on a narrow region, as a result of the chaos component in the classical phase space. Relatively few values of the tunneling rate, however, spread outside the clustering region due to the integrable component. Resonant tunneling of electrons in nonhyperbolic chaotic graphene system was found to exhibit a similar behavior. The analytic solutions of the Dirac equation in certain integrable domains, in combination with physical arguments, can be exploited to gain a theoretical understanding of these phenomena. In particular, the Dirac equation in one dimension and in a circular ring domain can be solved analytically, based on which the concept of self-energies can be used to calculate the tunneling rate. Because relatively few and distinct classical periodic orbits are present in the integrable component, the corresponding relativistic quantum states can have drastically different behaviors, leading to a wide spread in the values of the tunneling rate. In contrast, the chaotic component has embedded within itself an infinite number of unstable periodic orbits, which provide far more quantum states for tunneling. Due to the nature of chaos, these states are characteristically similar, leading to clustering of the values of the tunneling rate to a narrow band.

Resonant tunneling in graphene systems in the presence of chaos and electron-electron interactions. In realistic physical systems, many-body interactions, especially electron-electron interactions, can lead to many striking phenomena of fundamental importance. A resonant tunneling system made of graphene taking into account the electron-electron interactions can be described by the Hubbard Hamiltonian. A large number of eigenenergies and eigenstates were then calculated [136], revealing a class of localized states with near-zero tunneling rate in the integrable systems. These states are not the edge states typically seen in graphene systems, but the consequence of many-body interactions. A physical analysis based on solutions of the Dirac equation confirmed that this is indeed the case [136]. It was then demonstrated that, when the geometry of the system is modified to one with chaos, the localized states are effectively removed, implying that in realistic situations where many-body interactions are present, classical chaos is capable of facilitating quantum tunneling. These results on the interplay among relativistic quantum mechanics, many-body interactions, and classical chaos, besides their importance to fundamental physics, can be useful for developing nanoscale devices such as graphene-based resonant-tunneling diodes.

\section{B. Effect of fully developed chaos on relativistic quantum tunneling}

\section{Calculation of tunneling rate in relativistic quantum systems}

We consider the setting of resonant tunneling in which a potential barrier $U(\boldsymbol{r})$ with height $U_{0}$ divides a symmetric cavity into two equal parts. The ratio of the width of the barrier and the width of the left part is much smaller than one (e.g., 1/24). For nonrelativistic quantum resonant tunneling, due to the geometric symmetry in the double-well systems, the eigenstates are either symmetric or antisymmetric about the central barrier. A symmetric/antisymmetric pair means that, on one side of the double well, the eigenfunctions completely overlap, while on the other side, the eigenfunctions differ by the factor of -1 . The tunneling rate can then be defined as the energy splitting $\Delta E$ between the symmetric and antisymmetric eigenstate pairs [134, 180, 181], which can be seen, as follows. Let $\psi_{S}$ and $\psi_{A}$ denote a symmetric and antisymmetric eigenstate pair. 

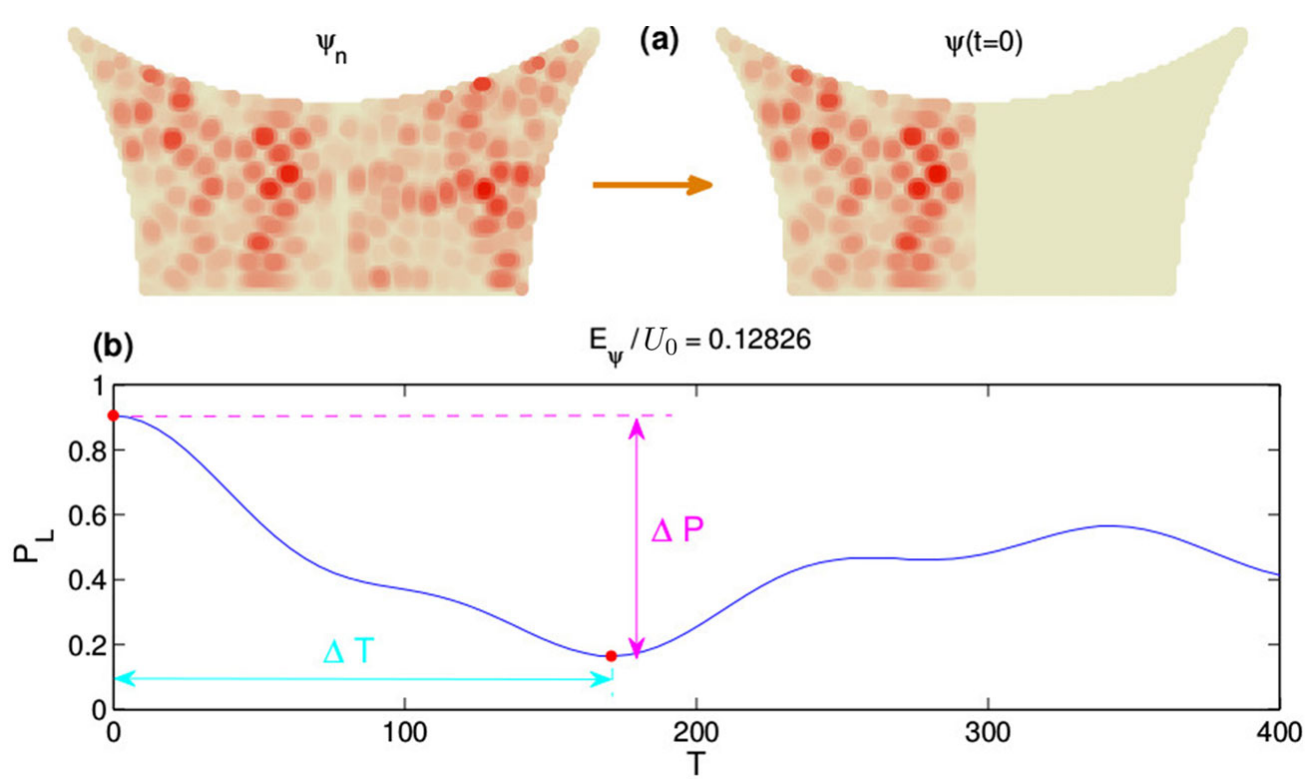

FIG. 49. A general method to calculate the tunneling rate in Dirac fermion or graphene systems. (a) For any eigenstate $\psi_{n}$, one renormalizes the spinor wavefunction localized to the left of the potential barrier, which leads to a new wavefunction $\psi(t=0)$. (b) Time evolution of probability for particle to be observed in the left part of the billiard, $P_{L}(t)$. Tunneling rate is defined as $R \equiv 1 / \Delta T$.

Define a new state $\psi(t)=\psi_{S} e^{-i E_{S} t / \hbar}+\psi_{A} e^{-i E_{A} t / \hbar}$. At time $t=0, \psi(0)=\psi_{S}+\psi_{A}$ describes a state in which the particle can be found only on one side of the double well. Let this state evolve. At time $t_{0}=\pi \hbar / \Delta E$, where $\Delta E=\left|E_{S}-E_{A}\right|$, one has $\psi\left(t_{0}\right) \propto \psi_{S}-\psi_{A}$, which means that the particle has tunneled to the other side of the well.

The difficulty with Dirac fermion systems is that there is no clear criterion to separate the symmetric/antisymmetric pairs from other mixed states. A symmetric state is not necessarily paired with a corresponding antisymmetric state. Moreover, for massless Dirac fermions, because of the violation of the time-reversal symmetry [93], the reflection symmetry is broken. As a result, the eigenstates do not have to be symmetric or antisymmetric. In such a case, a more general definition of the tunneling rate for relativistic quantum systems is necessary.

A heuristic method to calculate the resonant tunneling rate for relativistic quantum systems was introduced [134]. For an arbitrary symmetric double-well system, one first picks a random linear combination of the eigenstates (spinors), denoted as $\psi=\sum_{n} a_{n} \psi_{n}(\boldsymbol{r})$, where typically $n$ runs over a small set, and it can be just one state. This is feasible because of the availability of methods that allow the eigenstates of the Dirac equation in an arbitrarily closed domain to be solved (Sec. II). An example is shown in Fig. 49(a), where only one eigenstate is selected. Next, one keeps only the left side of $\psi$, and sets the right side and the barrier part of $\psi$ to zero. The state is renormalized and it is denoted as $\bar{\psi}=\sum_{n} \bar{a}_{n} \psi_{n}^{L}(\boldsymbol{r})$, where $\bar{a}_{n}$ 's are the renormalized coefficients, and $\psi_{n}^{L}(\boldsymbol{r})=\psi_{n}(\boldsymbol{r})$ for $\boldsymbol{r}$ at the left well, and $\psi_{n}^{L}(\boldsymbol{r})=0$ otherwise. The state $\bar{\psi}$ is then allowed to evolve in time. It is convenient to express it in terms of the linear combination of all eigenstates: $\bar{\psi}=\sum_{m} b_{m} \psi_{m}(\boldsymbol{r})$, where the summing index $m$ runs through all eigenstates. The coefficients $b_{m}$ can be calculated as

$$
b_{m}=\int_{D} \psi_{m}^{*} \bar{\psi} \mathrm{d} \boldsymbol{r}=\int_{L} \psi_{m}^{*} \sum_{n} \bar{a}_{n} \psi_{n}^{L} \mathrm{~d} \boldsymbol{r}=\sum_{n} \bar{a}_{n} \int_{L} \psi_{m}^{*} \psi_{n} \mathrm{~d} \boldsymbol{r}
$$


where $D$ and $L$ are the integration domains of the whole double well and of the left well, respectively. The time evolution of the state $\bar{\psi}$ is given by $\bar{\psi}(t)=\sum_{m} b_{m} \psi_{m} e^{-i E_{m} t / \hbar}$. Typically only one eigenstate is selected, i.e., there is only one $\bar{a}_{n}$ that is nonzero. If all the states are symmetric or antisymmetric, there will be two nonzero $b_{m}$ 's only, e.g., $n$ and its corresponding antisymmetric or symmetric pair, which is the case for nonrelativistic quantum systems. For a relativistic quantum system, there are in general several nonzero $b_{m}$ 's. Because the particle state is initially confined within the left well, to characterize the tunneling process of this state, one can calculate the probability that the particle is found in the left well with respect to time: $P_{L}(t)=\int_{L}|\bar{\psi}(t)|^{2} \mathrm{~d} \boldsymbol{r}$. An example of the probability evolution is plotted in Fig. 49(b). The tunneling rate $R$ is found at the time when $P_{L}(t)$ reaches minimum for the first time, i.e., $R \equiv 1 / \Delta T$, as shown in Fig. 49(b). This definition is general because, for resonant tunneling in nonrelativistic quantum systems where symmetric/antisymmetric eigenstate pairs do exist, it reduces to $\Delta E$ used in, e.g., Ref. [180].

2. Relativistic quantum resonant tunneling in integrable and fully chaotic double potential well systems

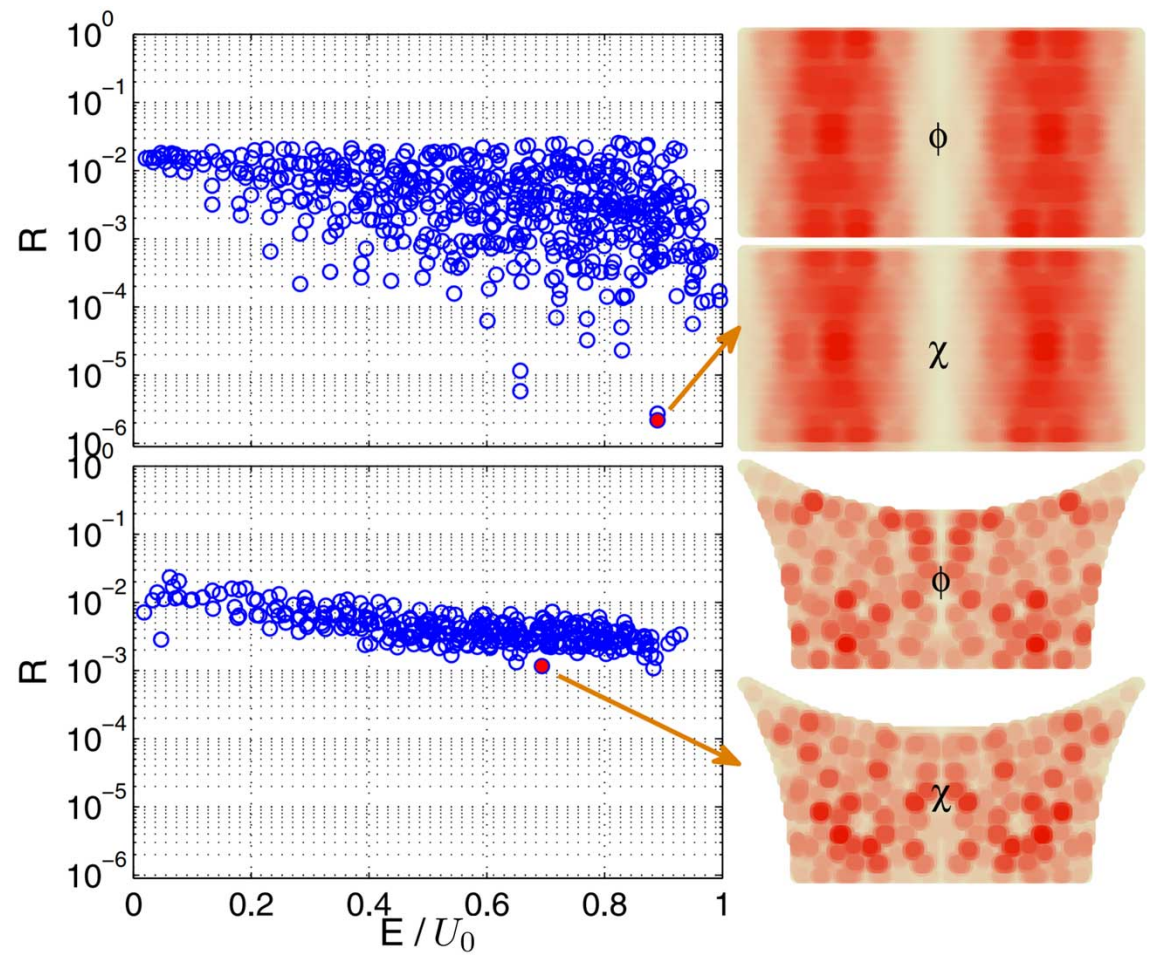

FIG. 50. Tunneling rates and patterns of local density of states for massless Dirac fermion in integrable and chaotic double-well systems. The Dirac spinor has two components, denoted as $\phi$ and $\chi$. The barrier height $U_{0}$ is about 60 in the unit system $\hbar=c=1$. For the rectangle double well, the width and the height are set to be 2 and 1, respectively. The bow-tie chaotic shape is obtained by cutting the rectangle with three arcs such that the cut parts are all 0.3 measured on both sides of the baseline and central vertical line of the rectangle. The ratio of the left well width to the barrier width is $24: 1$ for the rectangle. From Ref. [134] with permission.

The effects of fully developed chaos on relativistic quantum resonant tunneling were stud- 
ied [134]. Figure 50 shows the generalized tunneling rate $R$ versus the normalized energy $E / V_{0}$ for a massless Dirac fermion in the double-well barrier system for two types of geometry: one classically integrable and another fully chaotic. For the integrable geometry, there are states with extremely low tunneling rates, as indicated by the arrow in the upper panels of Fig. 50. These correspond to the states localized nearly entirely in the left or right side of the potential barrier, which "survive" the tunneling process between the two sides, as indicated by the accompanying pattern of local density of states (LDS). In nonrelativistic quantum transport, these are effectively quantum pointer states $[156-158,436]$. In relativistic quantum systems, both components of the underlying Dirac spinor exhibit a heavy concentration of the probability in orbits along which particles travel vertically back and forth on either side, parallel to the barrier. For the fully chaotic geometry, while signatures of pointer states can still be found, they are weak as compared with those in the integrable counterpart, as shown by the LDS patterns in the lower panels of Fig. 50. Analogous behaviors occur when the entire cavity is made of graphene, as shown in Fig. 51. Thus, for both Dirac fermion and graphene systems, classical chaos can greatly suppress the spread in the quantum tunneling rate, as in nonrelativistic quantum systems [180].

A phenomenon common to Dirac-fermion and graphene resonant tunneling systems is that, for small energies, pointer states are far less likely than in nonrelativistic quantum systems. In fact, in both types of relativistic quantum systems, the tunneling rate can be quite large even in the small energy regime, as shown in Figs. 50 and 51. This is the direct consequence of Klein tunneling $[154,265,266]$, which finds no counterpart in nonrelativistic quantum mechanics where the tunneling rate tends to zero as the energy is decreased to zero [180]. Although both Dirac fermion and graphene systems show almost identical relativistic behaviors at low energies and in both systems the phenomenon of chaos regularization can occur, there is still a difference between them. In particular, the Dirac equation describes a single massless Dirac fermion. However, for a graphene system in the low energy limit, the two electron states at different atoms (commonly called $A$ and $B$ ) in a unit cell can be regarded of as the two states of a massless quasi-particle's pseudo-spin, and there are in fact two such massless particles, due to the two distinct Dirac points. In the presence of a spatially short-range potential, there is coupling between the two Dirac points.

\section{Relativistic quantum tunneling in nonhyperbolic chaotic systems}

\section{Nonhyperbolic dynamics in the classical limit}

The mushroom billiard exhibits a mixed phase space and the distribution of the recurrence time is algebraic, a typical characteristic of nonhyperbolic chaotic Hamiltonian systems [171, 437]. It is one of the few Hamiltonian systems for which these features can be proven rigorously [435], and the corresponding nonrelativistic quantum tunneling dynamics was studied [374]. As shown in the top row of Fig. 52, in the classical limit, there are stable and marginally stable periodic orbits, as well as chaotic orbits. The study of the phase-space dynamics is facilitated by the fact that the collisions with the billiard walls follow the simple rule of reflection, so a discrete-time billiard map can be derived with two dynamical variables: the boundary arc length from the left corner of the mushroom cap and the incident angle of the collision point. Representative phase-space maps are shown in the bottom panel of Fig. 52. There are two regions in the physical space responsible for the integrable dynamics: one corresponding to the semicircle $\pi R$ and the two boundary segments $R-r$ in which there are periodic orbits around the mushroom cap (type-I orbit in Fig. 52), and 


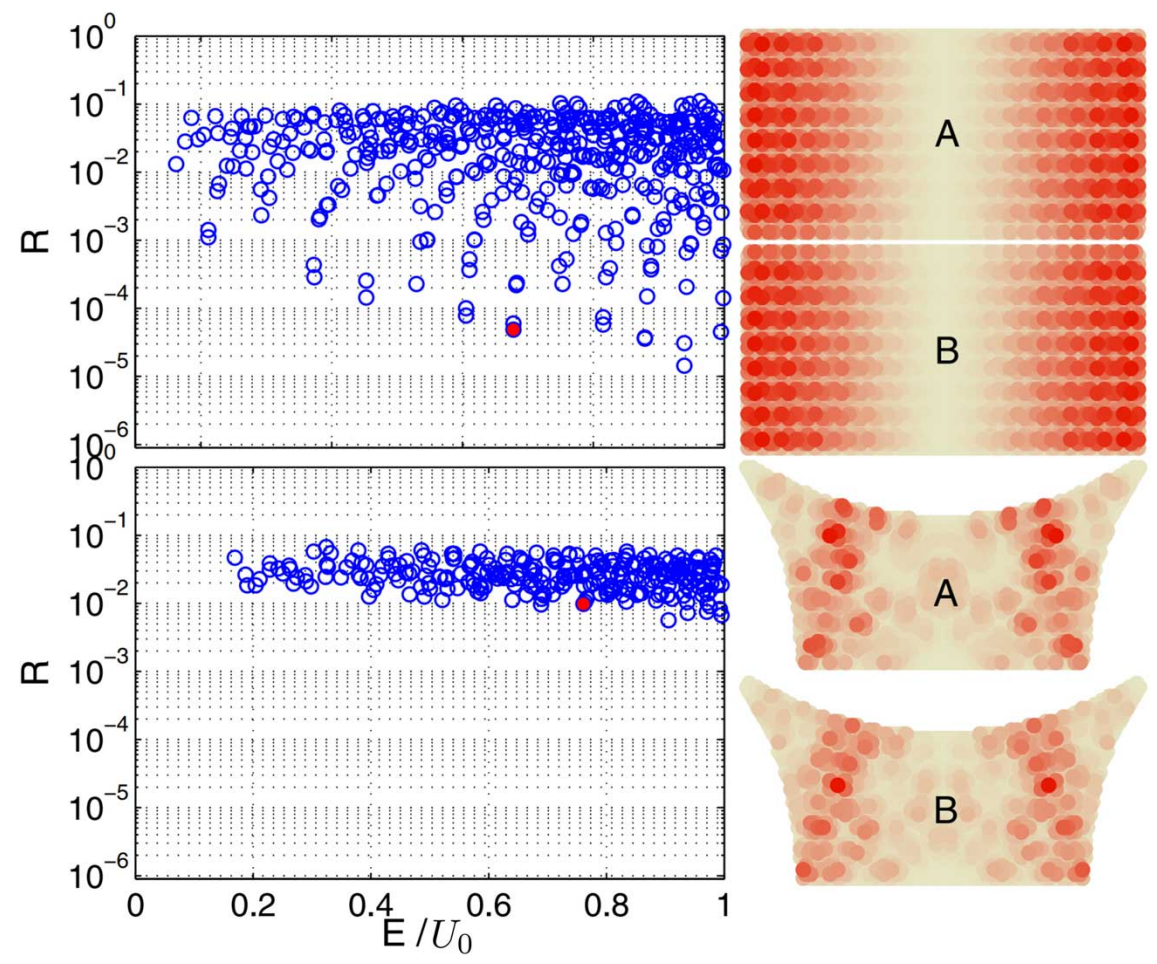

FIG. 51. Tunneling rates and LDS patterns in integrable and fully chaotic graphene double-well systems. For graphene, there are two distinct types of atoms in a unit cell, denoted as $A$ and $B$. The tight-binding model was used to calculate the eigenstates. The barrier height is fixed at $U_{0} \sim 0.67 t$. After converting to the $\hbar=c=1$ unit system, the geometric measures and the barrier potential are the same as those for Fig. 50. From Ref. [134] with permission.

another located within the two boundary segments $h$ for small incident angles (type-II marginally stable periodic orbit in Fig. 52). The area of the latter in the billiard map increases with the length of the mushroom stem. When the incident angle becomes sufficiently large on the stem part (or small on the cap part) of the boundary, the trajectories are chaotic, covering both parts of the mushroom, as shown as the type-III orbit in Fig. 52.

2. Relativistic quantum manifestation of nonhyperbolic classical dynamics: clustering of tunneling rate

Figure 53 shows the relativistic quantum tunneling rates versus the energy for a mushroom double-well system. Four representative points of different levels of tunneling are identified, which correspond to four characteristically different classical orbits in the mushroom billiard. In particular, the group of points with the highest tunneling rates, e.g., the red dot marked by (a), are associated with the states concentrating in the stem of the mushroom, as shown by the associated eigenstates in Fig. 53(a). These states correspond to the classical trajectories of type-II in Fig. 52. Figure 53(b) shows another type of states, those that circulate around the mushroom cap, which correspond to the classical trajectory of type-I in Fig. 52. The tunneling rates for this class of states are somewhat intermediate. Figure 53(c) shows an eigenstate with a relatively low tunneling rate, 

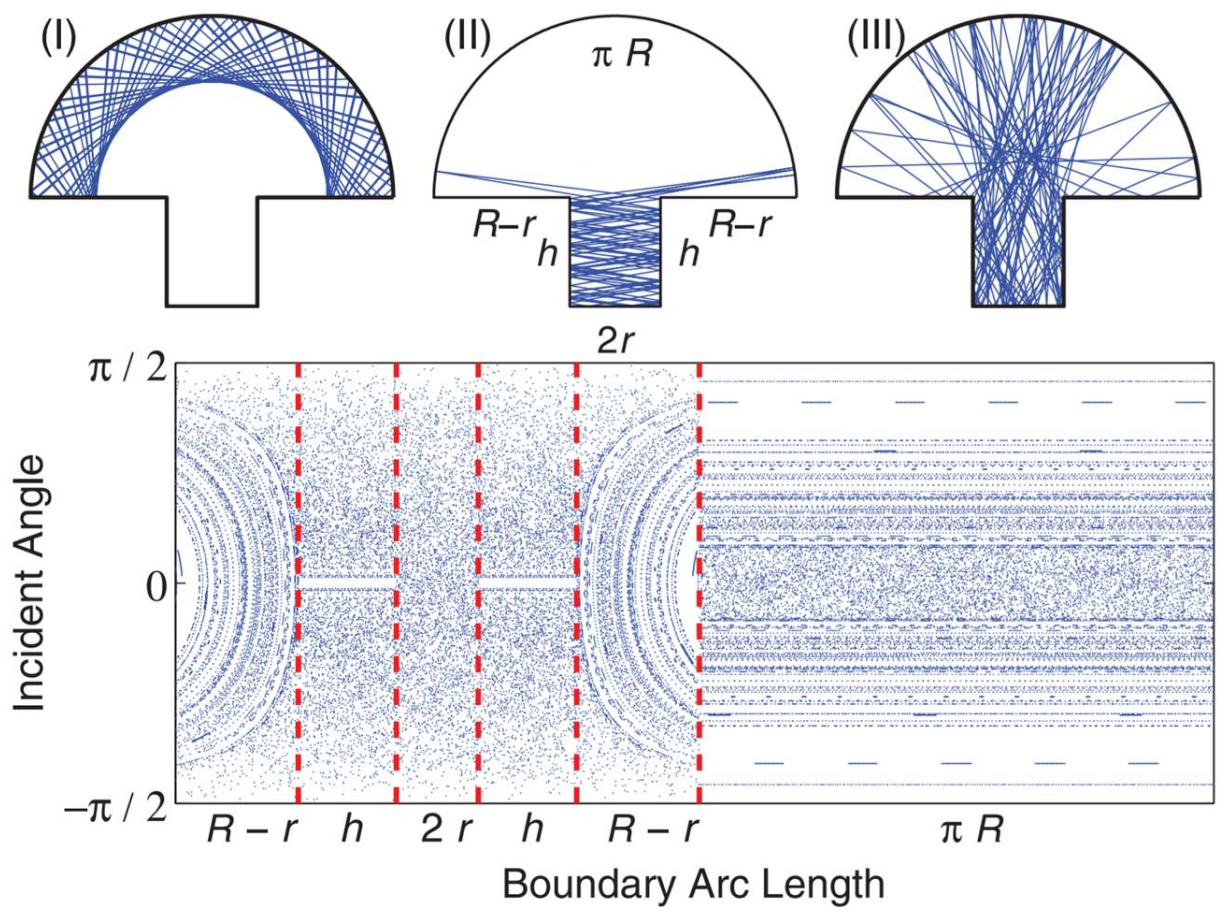

FIG. 52. Nonhyperbolic classical dynamics of the mushroom billiard. The mushroom billiard consists of a semicircular cap and a rectangular stem. The top row show three different types (I, II, III) of classical trajectories: stable, marginally stable periodic, and chaotic orbits. The bottom panel depicts the discrete time map in the phase space defined by the boundary arc length and the incident angle. The regions separated by the red vertical dashed lines correspond to the line/arc boundary segments marked in (II). From Ref. [135] with permission.

which is a state localized on the narrow regions near but parallel to the potential barrier, leading to extremely small probability for the state to cross the barrier. No classical periodic orbits can be identified to correspond to such states [135], due to the potential barrier along the symmetric line of the billiard. Overall, the cases in Figs. 53(a-c) correspond to the integrable component of the classical dynamics. Because of the large variation in the structure of the classical orbits in the integrable component, there is a wide spread in the relativistic quantum tunneling rate. In contrast, eigenstates corresponding to chaotic orbits are "similar" in the sense that they tend to spread over the entire classically allowed region in the billiard domain. As a result, the variation in their tunneling rates is significantly reduced. One such eigenstate is shown in Fig. 53(d). Since the chaotic orbits are uncountable (versus countable orbits belonging to the integrable component), a large number of eigenstates with similar values of the tunneling rate exist, leading to clustering of the rates into a relatively narrow band, as indicated in Fig. 53. The clustering phenomenon persists when the geometry of the mushroom is varied [135], e.g., by decreasing or increasing the size of the rectangular stem relative to the radius of the semi-circle. For those cases, an examination of the corresponding classical phase map revealed that shrinking the width of the mushroom stem makes regular trajectories bouncing between the two walls of the stem more pronounced [135]. This leads accordingly to more pronounced relativistic quantum eigenstates. In contrast, enlarging the stem width makes the formation of such "stem states" more difficult, leading to values of the tunneling rate significantly smaller than those in the clustered band. 

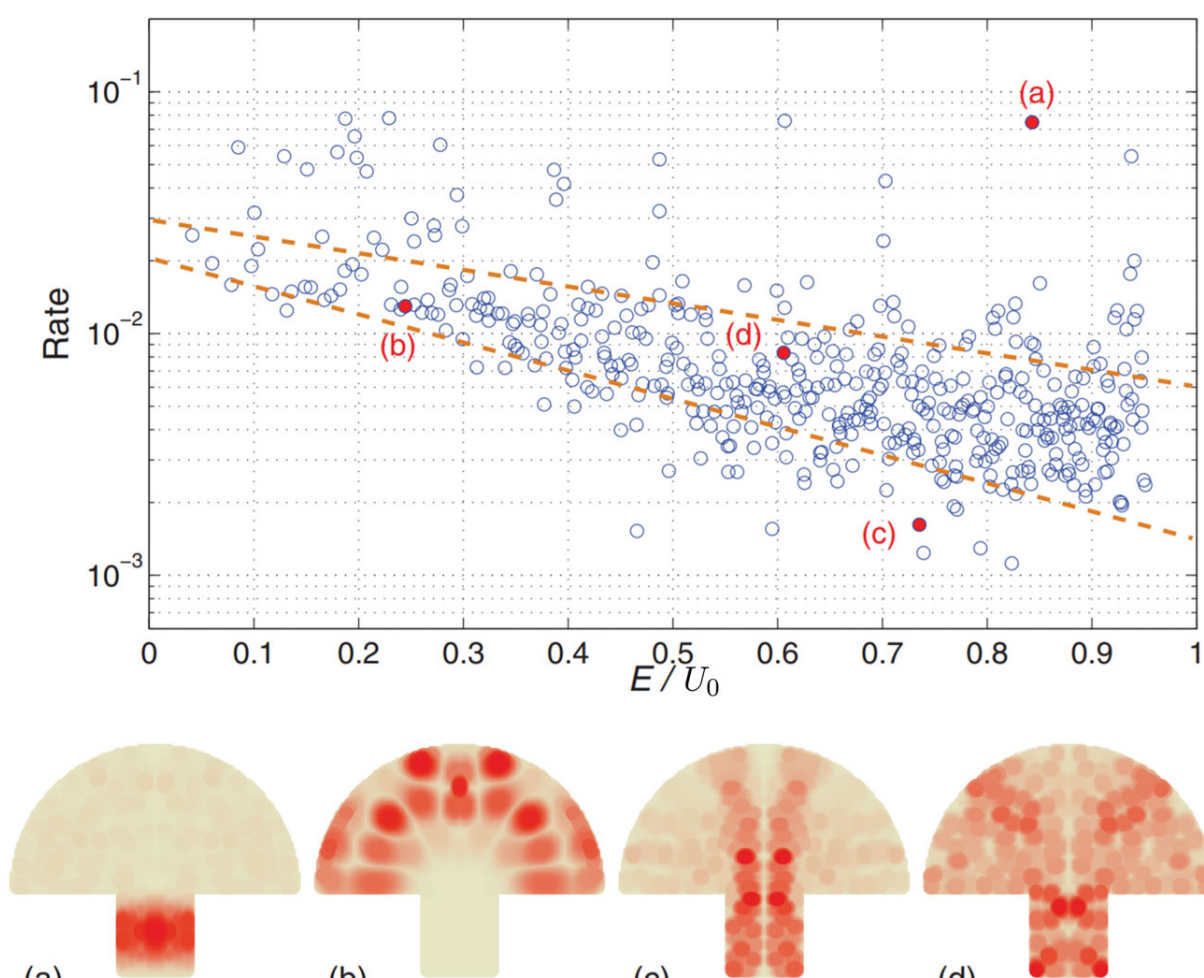

(a)

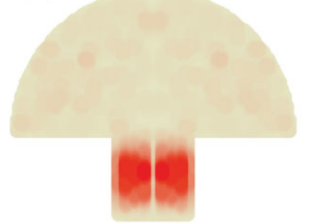

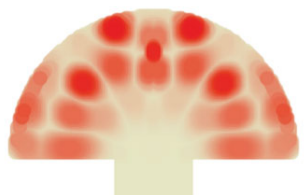

(b)

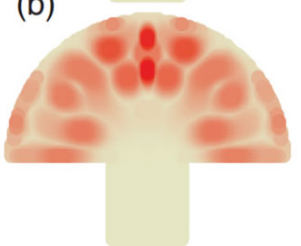

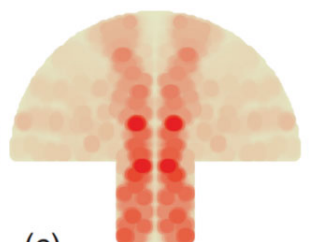

(c)

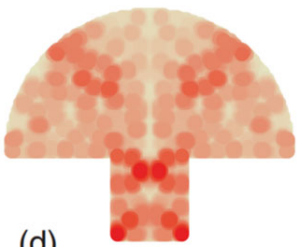

(d)

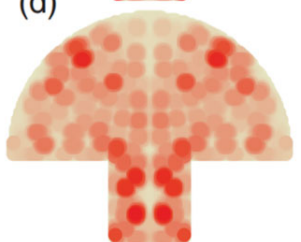

FIG. 53. Typical behaviors of tunneling rates and eigenstates for a massless Dirac fermion in the mushroom billiard. The billiard parameters are $r=0.25 R$ and $h=0.6 R$. The height and width of the potential barrier are $U_{0}=60$ and $w=0.15$, respectively. The first and second rows of patterns show the intensity distributions of the $\phi$ and $\chi$ components of the Dirac spinor, respectively. The four red points in the tunneling-rate plot (upper panel) correspond to the patterns for (a) a marginally stable periodic orbit of type-II, (b) a stable periodic orbit of type-I, (c) an orbit with extremely low tunneling rate, and (d) a chaotic orbit of type-III. From Ref. [135] with permission.

A similar clustering phenomenon occurs for mushroom billiard of the same geometry but made entirely of graphene, as shown in Fig. 54. The apparently similar tunneling phenomena presented in the graphene system, however, should not be mixed with those in the single massless Dirac fermion system. For treating graphene systems, field theoretical frameworks were developed [438440], as well as semiclassical approaches [441-444].

The placement of the clustering bands separated by the dashed lines in Figs. 53 and 54 are heuristic because, for any given small energy interval, the values of the tunneling rate associated with the chaotic orbits are "embedded" and "mixed" with some values due to the localized states in the integrable component. It is thus not possible to have a "clear-cut" of the values of the chaotic tunneling rate. Nonetheless, the clustering band has two features. First, the width of the band apparently increases with energy. This feature is in fact shared by nonrelativistic quantum tunneling in chaotic domains, where it was observed [180] that the variance of the tunneling rate 

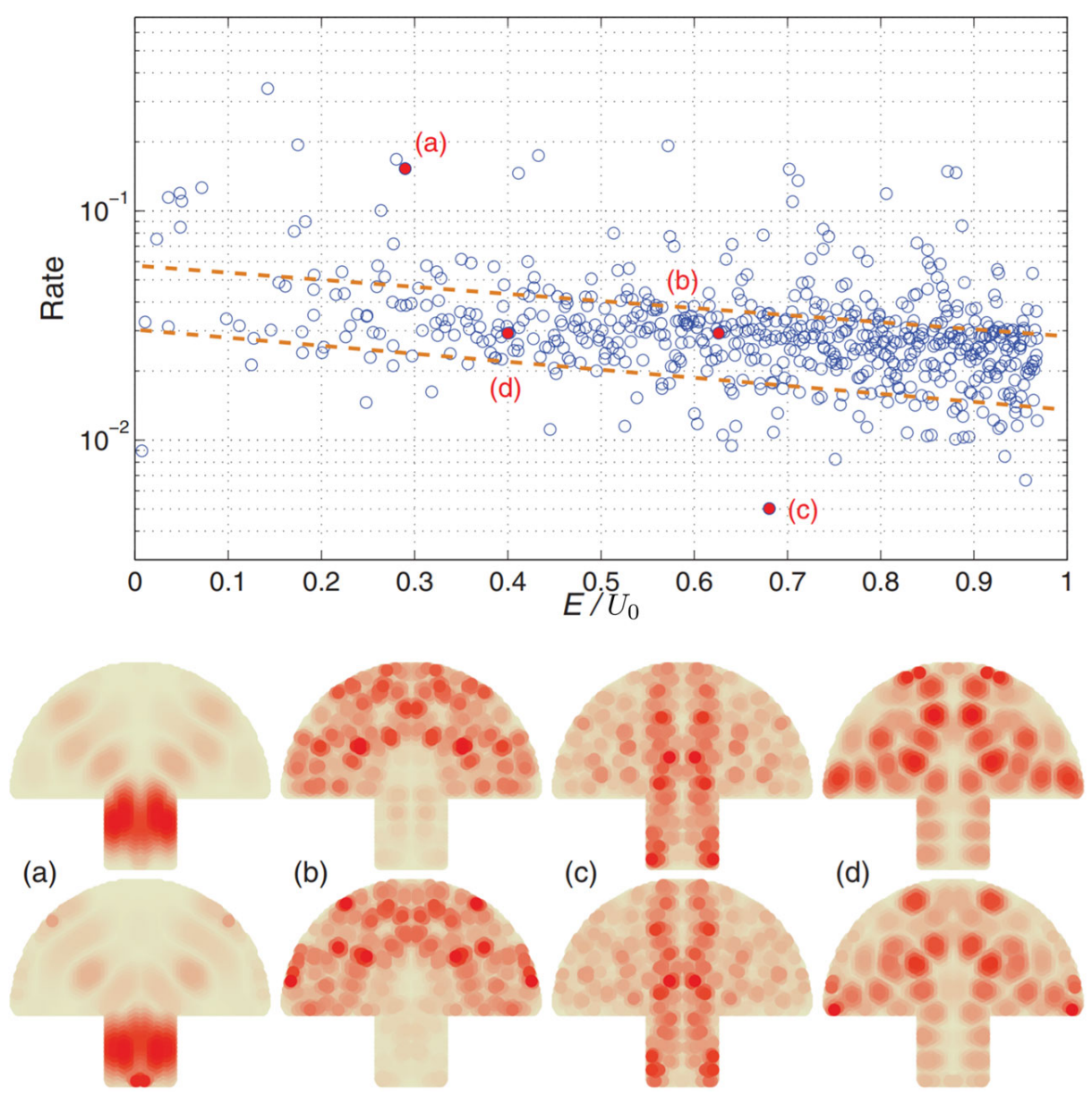

FIG. 54. Tunneling rates and representative eigenstates for mushroom billiard made entirely of graphene. The dimensions of the graphene system and the barrier height and width are proportional to those of the Dirac fermion tunneling system in Fig. 53, but the first and the second rows of patterns represent, instead of Dirac spinors, the $A$ and $B$ atoms in the graphene unit cell. The eigenenergies and eigenstates were calculated by using the standard tight-binding Hamiltonian for the closed graphene system. The legend is the same as that in Fig. 53. From Ref. [135] with permission.

in a moving window increases with the energy, which can be explained by using Berry's randomphase approximation [445] for eigenstates in a fully chaotic domain. Second, for $E \rightarrow 0$, the values of the tunneling rate do not approach zero, in dramatic contrast to nonrelativistic quantum tunneling. This is due to the unique relativistic quantum phenomenon of Klein tunneling [154].

\section{Physical theory for relativistic quantum tunneling in classically nonhyperbolic systems}

General framework. The concept of self-energy from quantum transport theory [369, 377] can be exploited to obtain a physical understanding of relativistic quantum tunneling in classically nonhyperbolic chaotic systems [135]. The mushroom domain consists of two closed symmetric wells coupled through the potential barrier along the line of symmetry. For a given state localized in one well, the tunneling rate is effectively the decay rate of Dirac fermion into the other well. For 
convenience, the left well can be denoted by superscript (1), and the barrier together with the right well by superscript (2), as shown in Fig. 55. The Dirac equations for the whole double-well system can then be written in terms of the Hamiltonians for regions (1) and (2), $H_{1}$ and $H_{2}$, respectively,

$$
\left[\begin{array}{ll}
H_{1} & V_{12} \\
V_{21} & H_{2}
\end{array}\right]\left[\begin{array}{l}
\psi^{(1)} \\
\psi^{(2)}
\end{array}\right]=E\left[\begin{array}{l}
\psi^{(1)} \\
\psi^{(2)}
\end{array}\right],
$$

where $V_{i j}$ are the coupling matrices. If the left well were itself closed, i.e., if it were surrounded by an infinitely high potential, the equation would become $H_{1} \psi^{(1)}=E_{1} \psi^{(1)}$. For a finite potential, the effect of coupling with the right well can be treated as an equivalent self-energy term $\Sigma^{R}$ :

$$
\left(H_{1}+\Sigma^{R}\right) \psi^{(1)}=E \psi^{(1)}
$$

where

$$
\Sigma^{R}=V_{12} G^{R} V_{21}
$$

is the self-energy due to the barrier and the right well, and

$$
G^{R}=\frac{1}{E+i \eta-H_{2}}
$$

is the retarded Green's function for region (2). The self-energy term is generally non-Hermitian, leading to complex energy values [377]. For each eigenstate of the left well, the energy shift can be obtained through the first-order perturbation theory as

$$
\left\langle\Sigma^{R}\right\rangle=\left\langle\psi^{(1)}\left|\Sigma^{R}\right| \psi^{(1)}\right\rangle
$$

which is typically complex. The real part of the energy shift changes the oscillating frequency of the corresponding eigenstate, while the imaginary part, denoted by $\gamma$, introduces a decay factor $\exp \left(-c_{0} \gamma t / \hbar\right)$ in the time evolution of the probability, which describes the escaping rate of the Dirac fermion from the left well to the right. Since the whole system is still closed, $\gamma$ only describes the transient event that the particle tunnels from left to right, whereas recurrences from right to left are ignored. If one lets the right well be infinitely long so that there is no reflective wave, the situation becomes a single left well coupled with an semi-infinite electron waveguide (or lead) through the potential barrier, and $\gamma$ will then be the tunneling rate for the single left-well system.

For nonrelativistic quantum tunneling, the self-energy $\Sigma^{R}$ and the complex eigenvalues of the non-Hermitian Hamiltonian $H_{1}+\Sigma^{R}$ can be calculated through the non-equilibrium Green's function formalism. Even for graphene described by a tight-binding Hamiltonian, this approach can be used. However, for open fermion system described strictly by the Dirac equation, a Green's function formulation is yet to be developed. Thus for a general geometry such as the nonhyperbolic mushroom billiard or even a domain in which the classical dynamics are fully chaotic, the tunneling rate $\gamma$ cannot be calculated analytically. Nonetheless, for certain integrable geometry, $\gamma$ can be calculated. To gain insights, a one-dimensional analytic example was studied [135] to illustrate the calculation of $\gamma$. Furthermore, the example can be used to show the agreement among the three methods to calculate the tunneling rate based on: (1) $\Delta T$, (2) $\Delta E$, and (3) $\gamma$. A two-dimensional analysis can then be carried out to demonstrate the wide spread in the tunneling rate for classically integrable domains. 


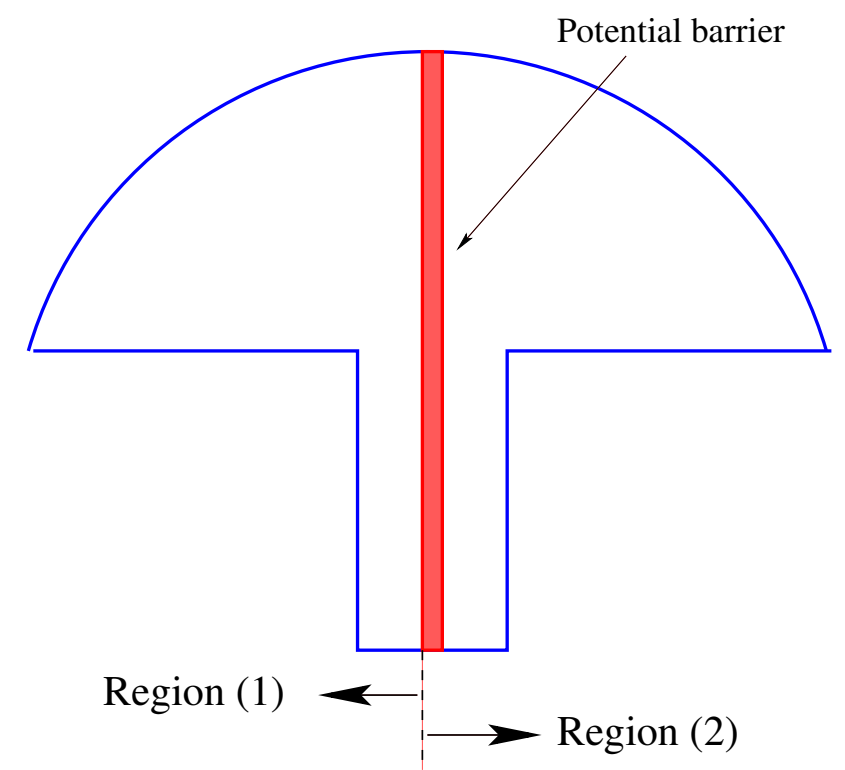

FIG. 55. Setting for developing a physical theory for relativistic quantum resonant tunneling in the mushroom billiard system. Shown is a schematic diagram of the division into subregions for theoretical calculation of the tunneling rate.

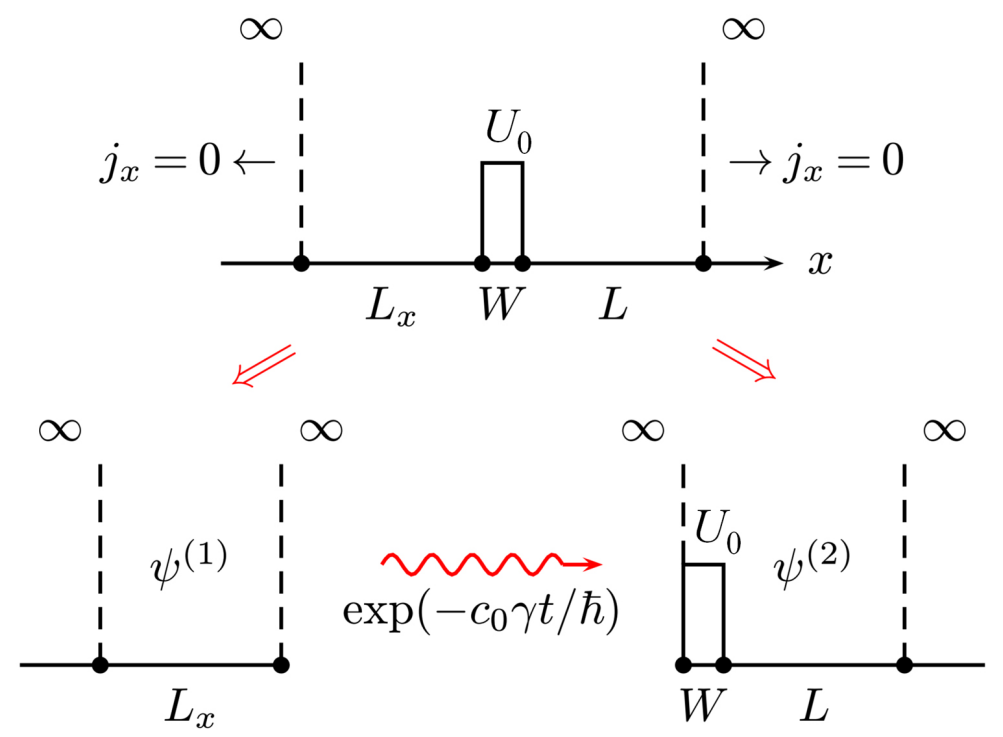

FIG. 56. One-dimensional "toy" resonant tunneling model. Schematic diagram for one-dimension tunneling of a massless Dirac fermion.

One-dimensional example. In one spatial dimension where a narrow potential barrier of finite height is placed at the center of an infinite potential well, the tunneling rate can be calculated exactly, with the result that it does not depend on the energy. In particular, consider the system shown in Fig. 56. Despite its simplicity, the system possesses some important properties of relativistic quantum tunneling such as the large tunneling rates for small energies due to the phenomenon of 
Klein tunneling. The starting point is to solve the one-dimensional Dirac equation

$$
\left(-i \hbar c \sigma_{x} \partial_{x}\right) \psi=(E-U) \psi
$$

separately for the two closed systems. For the left well of width $L_{x}, U=0$, so the solution is

$$
\psi_{n}^{(1)}(x)=\frac{1}{\sqrt{L_{x}}} \exp \left(i \frac{\pi}{4}\right)\left[\begin{array}{c}
\cos \left(k_{n} x-\frac{\pi}{4}\right) \\
i \sin \left(k_{n} x-\frac{\pi}{4}\right)
\end{array}\right],
$$

where $k_{n}=(n+1 / 2) \pi / L_{x}$. For the right part, the barrier has width $W$ and $U=V_{0}$, and the right well has width $L$ and $U=0$. One obtains the solution

$$
\psi_{n}^{(2)}(x)=\left\{\begin{array}{l}
A_{1} e^{i \kappa_{n} x} u_{+}+A_{2} e^{-i \kappa_{n} x} u_{-}, \text {barrier } \\
A_{3} e^{i k_{n} x} u_{+}+A_{4} e^{-i k_{n} x} u_{-}, \text {right-well },
\end{array}\right.
$$

where $k_{n}=E /(\hbar c)=\left[U_{0} W /(\hbar c)+(m+1 / 2) \pi\right] /(L+W), \kappa_{n}=k_{n}-U_{0} /(\hbar c), u_{+}=(1,1)^{T}$ and $u_{-}=(1,-1)^{T}$ are bases for the spinor, and the coefficients $A_{i}$ are determined by the boundary conditions. This is a combined solution for $E<U_{0}$ and $E>U_{0}$. The junction between the left and right wells where the coupling occurs is the region of interest. What is needed is a Green's function on a discrete lattice, having lattice points spaced by $a$, between two points along the $x$-axis. The energy shift can be expressed in the discrete lattice as

$$
\left\langle\Sigma^{R}\right\rangle=\psi_{n}^{(1) \dagger}\left(L_{x}-a\right) V_{12} G^{R}\left(L_{x}+a, L_{x}+a\right) V_{21} \psi_{n}^{(1)}\left(L_{x}-a\right),
$$

where $x=L_{x}$ is the junction between the left well and the barrier, and the coupling is given by $V_{12}=V_{21}^{\dagger}=-i \hbar c \sigma_{x}$. It is necessary to compute the Green's function

$$
G^{R}\left(L_{x}+a, L_{x}+a\right)=\sum_{n} \frac{\psi_{n}^{(2)}\left(L_{x}+a\right) \psi_{n}^{(2) \dagger}\left(L_{x}+a\right)}{E+i \eta-\hbar c k_{n}} .
$$

To eliminate the reflection back to the left well, one can let the right well be infinitely long, i.e., $L \rightarrow \infty$, so the summation changes to an integration

$$
\sum_{k} \rightarrow \frac{L+W}{\pi} \int \mathrm{d} k
$$

Note that $\left|A_{1}\right|^{2}=1 /[4(L+W)]$, which cancels the $L+W$ factor in Eq. (195). The value of the Green's function at $x=L_{x}+a$ becomes

$$
G^{R} \rightarrow \frac{1}{2 \pi} \int_{0}^{\infty} d k \frac{I_{2}+M_{k}}{E+i \eta-\hbar c k},
$$

where $I_{2}$ is the $2 \times 2$ identity matrix and

$$
M_{k}=\left[\begin{array}{rr}
\sin (2 \kappa a) & i \cos (2 \kappa a) \\
-i \cos (2 \kappa a) & -\sin (2 \kappa a)
\end{array}\right] .
$$

The integral over $I_{2}$ diverges, but the imaginary part of the integral is finite, which eventually contributes to $\gamma$. This imaginary contribution turns out to be $-\hbar c \eta /\left(2 \pi L_{x} E\right)$. To calculate the integral for the components of $M_{k}$, one can define

$$
\begin{aligned}
& S=\int_{0}^{\infty} \mathrm{d} k \frac{\sin (2 \kappa a)}{E+i \eta-\hbar c k}, \\
& C=\int_{0}^{\infty} \mathrm{d} k \frac{\cos (2 \kappa a)}{E+i \eta-\hbar c k} .
\end{aligned}
$$


The rate $\gamma$ can be expressed in terms of these integrals as

$$
\gamma=-\frac{(\hbar c)^{2}}{2 \pi L_{x}}[\cos (2 k a) \operatorname{Im}(C)-\sin (2 k a) \operatorname{Im}(S)]-\frac{\hbar c \eta}{2 \pi L_{x} E} .
$$

Evaluating the integrals $S$ and $C$ and using $E \approx E_{1}=\hbar c k_{n}$, one gets

$$
\begin{aligned}
\gamma_{n}= & -\frac{\hbar c}{4 \pi L_{x}} \operatorname{Im}\left[2 \operatorname{Ci}\left(-2 k_{n} a-i \delta\right) \cos \left(4 k_{n} a-\frac{2 a U_{0}}{\hbar c}+i \delta\right)\right. \\
& \left.+\left[\pi+2 \operatorname{Si}\left(2 k_{n} a+i \delta\right)\right] \sin \left(4 k_{n} a-\frac{2 a V_{0}}{\hbar c}+i \delta\right)\right]-\frac{\hbar c \delta}{4 \pi a L_{x} k_{n}},
\end{aligned}
$$

where $\delta \equiv 2 a \eta /(\hbar c)$, and $\mathrm{Ci}(x)$ and $\mathrm{Si}(x)$ are cosine and sine integrals defined as

$$
\begin{aligned}
& \operatorname{Ci}(x)=\gamma_{0}+\ln (x)+\int_{0}^{x} \frac{\cos (t)-1}{t} \mathrm{~d} t, \\
& \operatorname{Si}(x)=\int_{0}^{x} \frac{\sin (t)}{t} \mathrm{~d} t,
\end{aligned}
$$

where $\gamma_{0}$ is the Euler-Mascheroni constant. For small values of $a$ and $\eta$, one has

$$
\operatorname{Im}\left[\operatorname{Ci}\left(-2 k_{n} a-i \delta\right)\right] \approx-\pi / 2[1+\Theta(\hbar c k-\eta)],
$$

where $\Theta(\cdot)$ is the Heaviside step function. For $k>\eta /(\hbar c)$, one obtains a constant expression for $\gamma$ :

$$
\gamma=\frac{\hbar c}{2 L_{x}}
$$

The coefficient $c_{0}$ in the exponential-decay factor $\exp \left(-c_{0} \gamma t / \hbar\right)$ can be determined by considering the symmetry of the double-well system. The components of the spinor solution $\psi=\left(\psi_{1}, \psi_{2}\right)^{T}$ can be divided into two types: symmetric and antisymmetric. It is convenient to set the origin at the the center of the double well, so for the first component $\psi_{1}$, symmetric and antisymmetric solutions require $\psi_{1}^{\prime}(0)=0$ and $\psi_{1}(0)=0$, respectively. The second component is symmetric when the first is antisymmetric, and vice versa. It is convenient to name the symmetry type according to the first component, and compute the energy levels for $E<U_{0}$. For symmetric and antisymmetric states, the energy levels are

$$
\begin{aligned}
& E_{S}=\frac{W V_{0} / 2+\left(n_{S}+1 / 4\right) \hbar c \pi}{L_{x}+W / 2} \\
& E_{A}=\frac{W V_{0} / 2+\left(n_{A}-1 / 4\right) \hbar c \pi}{L_{x}+W / 2},
\end{aligned}
$$

respectively, where $n_{S}, n_{A} \in \mathbb{Z}$. Because these energy levels are equally spaced, the energy spacing between symmetric and antisymmetric states is

$$
\Delta E=\frac{\hbar c \pi}{2 L_{x}+W}
$$

Comparing $\Delta E$ with the expression of $\gamma$ in Eq. (201) and noting that $L_{x} \gg W$, one obtains the coefficient $c_{0}=\pi$. Figure 57 shows both $\Delta E$ and $\pi \gamma$ in comparison with the results from direct numerical evaluation of $\Delta T$. It can be seen that $\pi \gamma$ calculated from the equivalent system using the self-energy method agrees with $\Delta E$ quite well, demonstrating that the tunneling rate is nearly constant for all energies in one dimension. Due to the unique relativistic quantum phenomenon of Klein tunneling, the tunneling rate remains large even for $E \rightarrow 0$. 


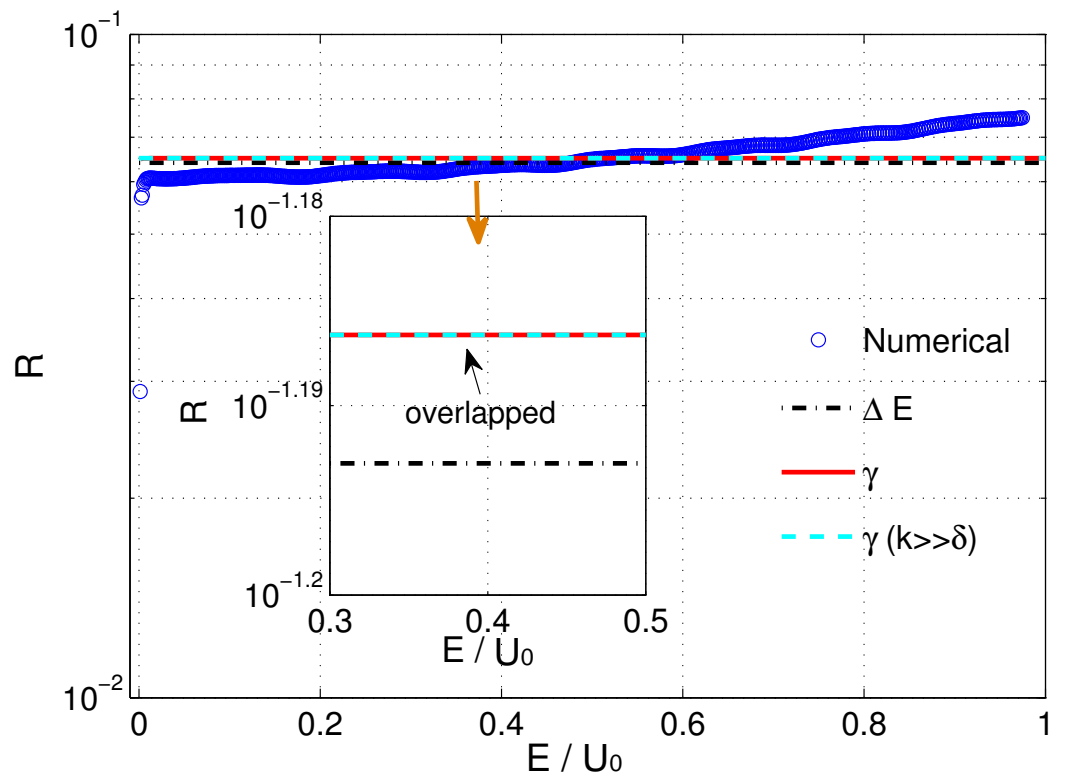

FIG. 57. Theoretical and numerical tunneling rates for a 1D massless Dirac fermion resonant tunneling system. Shown are two theoretical curves: $\Delta E$ from the energy spacings between symmetric/antisymmetric eigenstate pairs for the whole double-well system, and $\pi \gamma$ from the self-energy method. Inset shows a zoom-in view for $E / U_{0} \in[0.3,0.5]$, where the $\gamma$ curve overlaps with the $\gamma(k \gg \delta)$ curve.

Resonant tunneling in a two-dimensional Dirac fermion system with circular geometry. In two dimensions, analytic solution of the Dirac equation cannot be obtained in general, due to the entanglement of the two Cartesian coordinates in the first-order Dirac equation. The problem is solvable only for certain types of boundary conditions via separation of variables. One solvable case is the circular boundary condition. Figure 58 shows a ring with a thin concentric ring-shaped barrier in it, which makes it a double-well system. When all four radii are large, the system is topologically equivalent to a rectangular double well with periodic boundary condition in one direction. To solve the two-dimensional Dirac equation $(-i \hbar c \hat{\boldsymbol{\sigma}} \cdot \boldsymbol{\nabla}) \psi=(E-U) \psi$, one needs to use the polar coordinates $(r, \phi)$. With the relation $\partial_{x} \pm i \partial_{y}=\exp ( \pm i \phi)\left(\partial_{r} \pm i \partial_{\phi} / r\right)$, the general solutions can be written in the following form:

$$
\psi_{n}=e^{i n \phi}\left[\begin{array}{c}
Z_{n}(\lambda r) \\
\operatorname{sgn}(E-V) i e^{i \phi} Z_{n+1}(\lambda r)
\end{array}\right], n= \pm 1, \pm 2, \ldots
$$

where $Z_{n}(x)=A J_{n}(x)+B Y_{n}(x)$ is a linear combination of the first- and second-kind Bessel functions, and $\lambda=|E-U| /(\hbar c)$.

The ring double-well domain can be separated into two parts: the inner ring [denoted by superscript (1)] and the outer part [denoted by superscript (2)] consisting of the barrier and the outer ring. The asymptotic form of the Bessel functions can be used to obtain an analytical expression for the energy levels for $x \gg\left|n^{2}-1 / 4\right|$,

$$
\begin{aligned}
J_{n}(x) & \sim \sqrt{\frac{2}{\pi x}} \cos \left(x-\frac{n \pi}{2}-\frac{\pi}{4}\right), \\
Y_{n}(x) & \sim \sqrt{\frac{2}{\pi x}} \sin \left(x-\frac{n \pi}{2}-\frac{\pi}{4}\right) .
\end{aligned}
$$




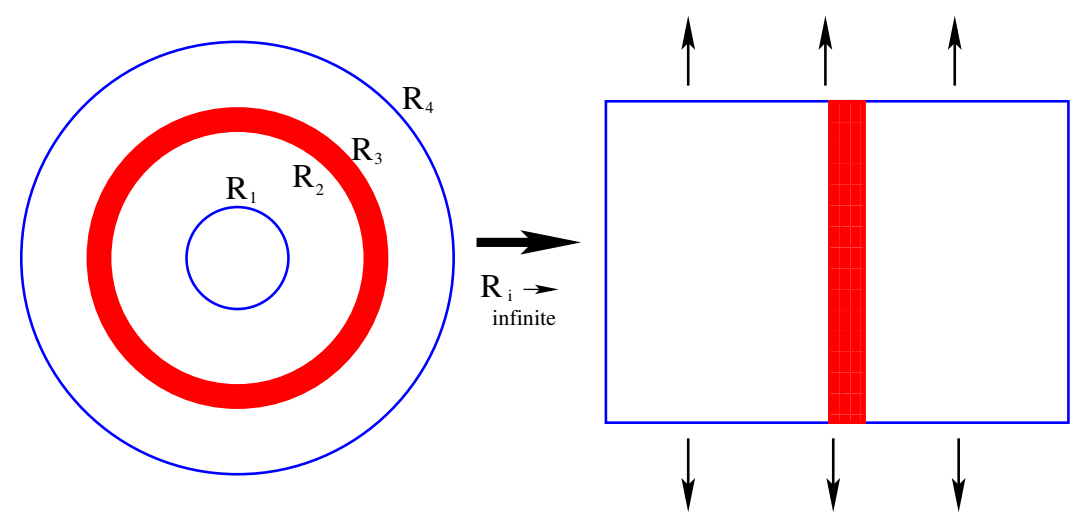

FIG. 58. Schematic picture of a two-dimensional ring domain for which the Dirac equation is analytically solvable. Topologically, for $R_{i} \rightarrow \infty$, the ring is equivalent to a rectangle with periodic boundary conditions in the vertical direction. The shaded regions denote the potential barrier of height $U_{0}$.

Applying two boundary conditions at $r=R_{1}, R_{2}$ and normalization leads to the solution for the inner ring for large radii:

$$
\psi_{m, n}^{(1)}=N \frac{1}{\sqrt{r}} e^{i n \phi}\left[\begin{array}{c}
\sin \left(k_{m}\left(r-R_{2}\right)+3 \pi / 4\right) \\
-i e^{i \phi} \cos \left(k_{m}\left(r-R_{2}\right)+3 \pi / 4\right)
\end{array}\right],
$$

where

$$
k_{m}=\frac{(m+1 / 2) \pi}{R_{2}-R_{1}},
$$

$m=0,1,2, \ldots$, and $N=\left[2 \pi\left(R_{2}-R_{1}\right)\right]^{-1 / 2}$. Since the eigenenergy $\hbar c k_{m}$ does not depend on the angular quantum number $n$, all different angular modes are degenerate for a single energy level. This happens when the ring is infinitely large, where the radial functions have the trigonometric form and the variation of the functions become periodic so that the energy levels are equally spaced. However, if the original radial solutions for the finite ring system were to be used, the energy levels would be found through the zeros of the integer-order Bessel functions, and thus have decreasing spacings. In that case, because the different integer-order Bessel functions differ in zeros, the eigenenergies are non-degenerate. For the outer part, the solutions combining $E<U_{0}$ and $E>U_{0}$ are

$$
\psi_{\ell, n}^{(2)}=\left\{\begin{array}{c}
N \frac{1}{\sqrt{r}} e^{i n \phi}\left[\begin{array}{r}
\sin \left(\kappa_{\ell}\left(r-R_{2}\right)+\pi / 4\right) \\
-i e^{i \phi} \cos \left(\kappa_{\ell}\left(r-R_{2}\right)+\pi / 4\right)
\end{array}\right] \\
N \frac{1}{\sqrt{r}} e^{i n \phi}\left[\begin{array}{r}
\sin \left(k_{\ell}\left(r-R_{4}\right)+3 \pi / 4\right) \\
-i e^{i \phi} \cos \left(k_{\ell}\left(r-R_{4}\right)+3 \pi / 4\right)
\end{array}\right],
\end{array},\right.
$$

where

$$
k_{\ell}=\left[\left(R_{3}-R_{2}\right) U_{0} /(\hbar c)+(\ell+1 / 2) \pi\right] /\left(R_{4}-R_{2}\right),
$$

and $\kappa_{\ell}=k_{\ell}-U_{0} /(\hbar c)$. Similar to one dimension, the Green's function at the coupling boundary $R_{2}+a$ is

$$
\left.G^{R}\left(r, \phi ; r, \phi^{\prime}\right)\right|_{r=R_{2}+a}=\sum_{n} \sum_{\ell} \frac{\psi_{\ell, n}^{(2)}(r, \phi) \psi_{\ell, n}^{(2) \dagger}\left(r, \phi^{\prime}\right)}{E+i \eta-\hbar c k_{\ell}}
$$


Letting the outer boundary be infinite, one can turn the summation over $k_{\ell}$ into an integration:

$$
G^{R} \rightarrow \sum_{n} \frac{e^{i n\left(\phi-\phi^{\prime}\right)}}{(2 \pi)^{2} R_{2}} \int_{0}^{\infty} \mathrm{d} k \frac{M_{k}^{\prime}}{E+i \eta-\hbar c k},
$$

where

$$
M_{k}^{\prime}=\left[\begin{array}{ll}
1+\sin (2 \kappa a) & i e^{-i \phi^{\prime}} \cos (2 \kappa a) \\
-i e^{i \phi} \cos (2 \kappa a) & e^{i\left(\phi-\phi^{\prime}\right)}[1-\sin (2 \kappa a)]
\end{array}\right]
$$

The energy shift is given by

$$
\left\langle\Sigma^{R}\right\rangle_{m}=\int \mathrm{d} \phi \int \mathrm{d} \phi^{\prime} \psi_{m, j}^{(1) \dagger}(\phi) V_{12} G^{R}\left(\phi ; \phi^{\prime}\right) V_{21} \psi_{m, j}^{(1)}\left(\phi^{\prime}\right)
$$

where $\psi_{m, j}^{(1)}\left(\phi^{\prime}\right)$ is evaluated at $r=R_{2}-a$. To calculate the coupling matrix, one uses

$$
\hat{\boldsymbol{\sigma}} \cdot \boldsymbol{\nabla}=\left[\begin{array}{cc}
0 & e^{-i \phi} \\
e^{i \phi} & 0
\end{array}\right] \partial_{r}+\left[\begin{array}{cc}
0 & -i e^{-i \phi} \\
i e^{i \phi} & 0
\end{array}\right] \frac{\partial_{\phi}}{r},
$$

and obtains the matrix as a function of $r$. The integral over the coordinates $\phi$ and $\phi^{\prime}$ is

$$
\int_{0}^{2 \pi} \mathrm{d} \phi \int_{0}^{2 \pi} \mathrm{d} \phi^{\prime} e^{i(j-n)\left(\phi-\phi^{\prime}\right)}=(2 \pi)^{2} \delta_{j n},
$$

so the summation over $n$ can be simplified. The final expression for the rate $\gamma$ is exactly the same as in one dimension, i.e., Eqs. (199) and (201). The reason for the identical rate $\gamma$ as in one dimension largely owes to the degeneracy of the angular modes. Because all tangent modes are degenerate for one specific energy level that depends on the radial function only, one cannot separate this system from a truly one-dimensional system.

While the two-dimensional solution is a special case where the large $r$ asymptotic form of the radial functions can be exploited, the general solution for circular boundaries is different because, for a certain energy level, the degenerate angular modes require an infinite $R_{i}$ condition. However, for finite rings, different angular modes are associated with different energy levels. Thus, the tunneling rates for a finite ring have a wide spread instead of a single straight line. In order to obtain the rate $\gamma$ for a finite-size ring, it is necessary to solve the eigenstates numerically. Using the general solution for a finite inner ring, Eq. (203), the normalization of the eigenstates, and the boundary conditions $Z_{n+1}\left(k_{m} R_{i}\right) / Z_{n}\left(k_{m} R_{i}\right)= \pm 1$ at $r=R_{i}(i=1,2)$, one can find the roots $k_{m}$ for each angular mode $n$ numerically. To eliminate reflection, it is necessary to set $R_{4}$ to be infinite. As a result, there are an infinite number of terms of $k_{\ell}$ in Eq. (208). A strategy to deal with the difficulty is to set a threshold $k_{\max }$ for the summation, and the criterion for choosing a proper value $k_{\max }$ is the quality of the orthogonality,

$$
\sum_{\left\{\ell: k_{\ell}<k_{\max }\right\}, n} \psi_{\ell, n}^{(2)}(\boldsymbol{r}) \psi_{\ell, n}^{(2) \dagger}\left(\boldsymbol{r}^{\prime}\right) \approx I_{2} \delta^{2}\left(\boldsymbol{r}-\boldsymbol{r}^{\prime}\right) .
$$

As the number of eigenstates $\ell$ below $k_{\max }$ increases, the computation burden increases as $\ell^{2}$. For fast computation, one can consider two approximated results for the outer part: one that uses the analytical solution of the Green's function in Eq. (209), and another that uses a relatively larger outer ring to numerically evaluate the rates. 


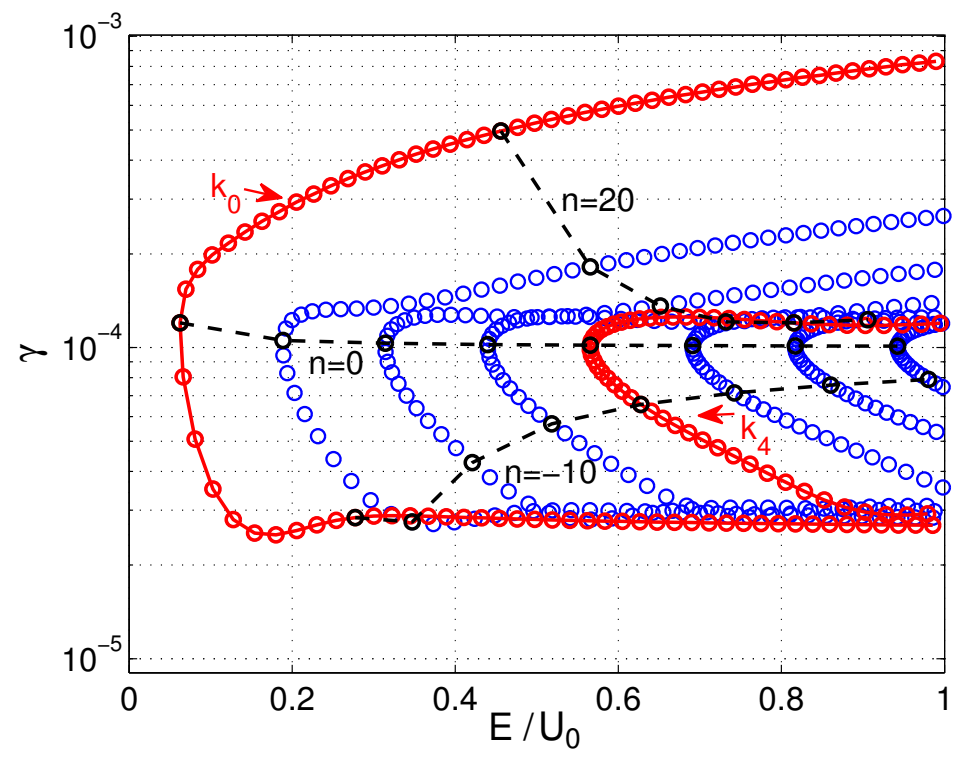

FIG. 59. Theoretical analysis of a two-dimensional, ring-type of relativistic quantum resonant tunneling system. Shown is the theoretical rate $\gamma$, where $n$ denotes the angular quantum number, and $k_{i}$ is the $i$ 'th energy level for all $n$. The eigenstates of the inner ring are found numerically, while the Green's function values for the outer part are calculated analytically. The radii and potential are $R_{1}=5, R_{2}=10$, $R_{3}=10.2, R_{4} \rightarrow \infty$, and $U_{0}=5$.

Some representative $\gamma$ values are shown in Fig. 59. It can be seen that the tunneling rates of the angular modes for the same energy level (e.g. $k_{0}$ and $k_{4}$ in Fig. 59) separate and spread into a wide range, instead of clustering together as a single point, which is the case in one dimension and two-dimensional rings with infinite radii. An alternative approach is to choose a large but finite value of $R_{4}$ and compute the tunneling rate $\gamma$ numerically. Again there is a wide spread in $\gamma$, and there are extremely small values of $\gamma$, corresponding to survival states from the opening process of the originally closed left well (inner ring) when a finite potential barrier is introduced into the system.

\section{Quantum tunneling in the presence of electron-electron interactions}

In most works in quantum chaos, the standard setting was that of single-particle quantum dynamics, whereas many-body effects such as electron-electron interactions were ignored. While there were previous studies of the interplay between many-body interactions and classical chaos [202-205, 446, 447], these were exclusively for non-relativistic quantum systems described by the Schrödinger equation. To investigate the effect of chaos on relativistic quantum systems with many-body interactions was thus an outstanding problem.

An initial attempt was made [136] to study relativistic quantum resonant tunneling in the presence of many-body interactions by using the standard Hubbard model with on-site repulsive Coulomb interactions for graphene systems. This paradigmatic model to treat interacting particles in a lattice was originally proposed [206] to describe the transition between conducting and insulating systems. For electrons in a solid, comparing with the conventional tight-binding model 
representing a single electron Hamiltonian, the Hubbard model contains a potential term to include the many-body effect through the mechanism of on-site Coulomb interaction [448, 449]. There has been a great deal of interest in the Hubbard model due to its relevance to frontier problems in condensed matter physics such as high-temperature superconductivity and the trapping of ultracold atoms in optical lattices [448]. It was found [136] that, while the Hubbard model is much more challenging and sophisticated than the tight-binding model, it can serve as a paradigm to gain significant physical insights into many-body relativistic quantum manifestations of distinct type of classical dynamics.

Resonant tunneling was studied [136] for four types of geometrical domains that lead to classically integrable (rectangle), nonhyperbolic (mushroom), or fully chaotic (stadium or bowtie) dynamics. The sizes were chosen such that the domains contain approximately the same number of carbon atoms. To gain physical insights, one can consider the class of integrable systems of rectangular shape. Since the whole system is closed, the eigenenergies and eigenstates can be calculated from the mean-field Hubbard Hamiltonian. A surprising finding was the emergence of a class of eigenstates with near zero tunneling rate [136]. In particular, for such an eigenstate, the spin-up and spin-down wavefunctions are typically separated, i.e., the spin-up electrons reside in only one potential well while the spin-down electrons reside in the other. As a result, if the initial state is spin-up in one potential well, it is localized and will stay in the same well practically for an infinite amount of time with little quantum tunneling. When the potential term characterizing the on-site Coulomb interactions is removed so that the Hamiltonian becomes that of the tight-binding type, such localized states no longer exist, indicating that they are the result of electron-electron interactions and consequently a distinct many-body phenomenon. An approximate theory can be derived based on the simplified picture of one-dimensional tunneling of massless Dirac fermions to explain the physical origin of the localized states [136]. When the geometrical shape is that of stadium or bowtie so that the classical dynamics are chaotic, the localized states are effectively removed and there is substantial tunneling. This means that, classical chaos is capable of destabilizing the localized states. For the mushroom domain that generate nonhyperbolic classical dynamics, due to the chaotic component, quantum tunneling can be regularized and enhanced. From the standpoint of device development such as graphene-based resonant-tunneling diodes, the localized states present an obstacle to effective tunneling and such states are therefore undesirable. From this perspective, classical chaos may be regarded as advantageous.

\section{Specification of integrable, fully chaotic, and nonhyperbolic domains}

For a meaningful comparison, four domains of approximately the same size were considered [136]. The geometric parameters of these domains are specified in Fig. 60. The size of the rectangular domain is $L=161 a=22.86 \mathrm{~nm}$ (armchair boundaries) and $D=85 a=12.05 \mathrm{~nm}$ (zigzag boundaries), where $a=1.42 \AA$. The total number of atoms is $N=10692$. For the stadium billiard, the parameters are $L=22.862 \mathrm{~nm}, D=12.052 \mathrm{~nm}$, and $N=9452$. The underlying classical dynamics are chaotic but with an infinite number of neutrally stable periodic orbits, corresponding to particles bouncing back and forth vertically at the rectangular portion of the billiard. The third type is a bowtie billiard, which is cut from a rectangle graphene sheet of $14.5 \mathrm{~nm}$ by $7.2 \mathrm{~nm}$ by circles of radius $r=32.7 \mathrm{~nm}$, and the domain contains 10946 carbon atoms. For the bowtie billiard, the classical dynamics are fully chaotic (hyperbolic) with all periodic orbits being unstable. The fourth type is a mushroom billiard with a mixed classical phase space (nonhyper- 

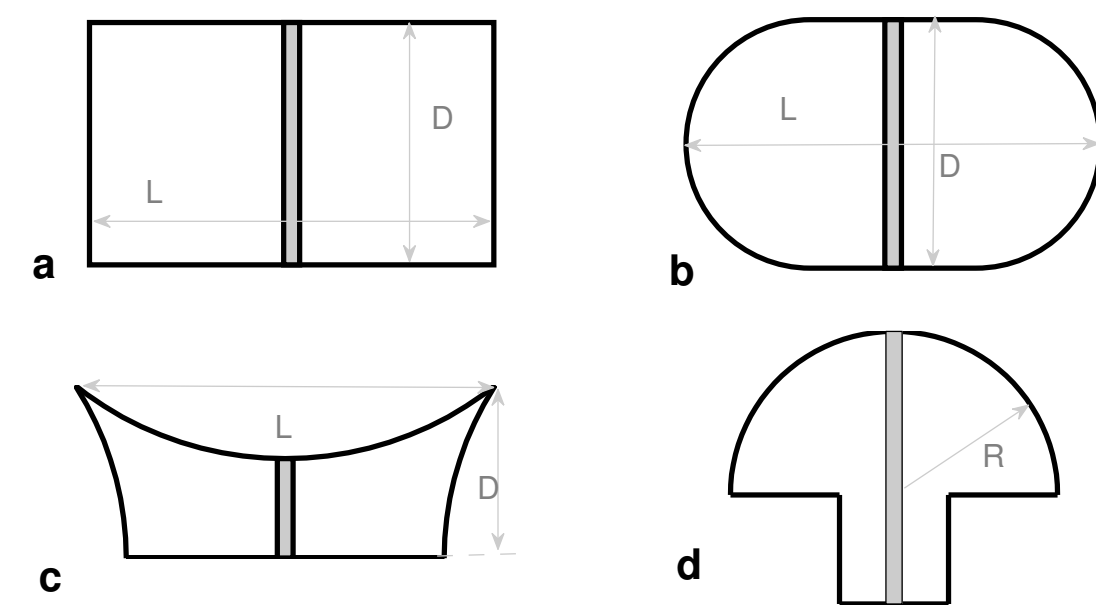

FIG. 60. Schematic illustration of four classes of geometrical domains for graphene billiards used to study the interplay among chaos, relativistic quantum mechanics, and many-body effect: (a) rectangle, (b) stadium, (c) bowtie, and (d) mushroom. The respective classical dynamics are integrable (a), chaotic with neutral periodic orbits (b), hyperbolic with all periodic orbits being unstable (c), and nonhyperbolic with a mixed phase space (d). The thin gray region along a symmetric line represents the potential barrier.

bolic classical dynamics $[135,435])$. The radius of the semicircle is $R=11.86 \mathrm{~nm}$, and the stem of the mushroom has the sizes $11.86 \mathrm{~nm}$ by $5.93 \mathrm{~nm}$. The total number of carbon atoms contained in the mushroom billiard is 10620 .

In the quantum regime, classical chaos is fundamentally suppressed due to the quantum uncertainty or finite Planck constant that effectively leads to "discretization" of the classical phase space. This should be contrasted to the discretization of the physical or configuration space through a crystal lattice. In general, any such discretization scheme is incompatible with chaos because classically, the Planck constant is effectively zero and motion can occur on arbitrarily fine scales, but this difficulty is somewhat alleviated due to the finite Planck constant in the quantum regime. Only in the limit of large geometric dimensions as compared to the lattice constant will the effect of chaos be manifested in the quantum behaviors. For the geometrical domains shown in Fig. 60, the pertinent quantum phenomena are quantitatively the same insofar as the system is sufficiently large, e.g., with more than 7000 atoms.

For all four types of billiards, a thin potential barrier is placed along the vertical symmetric line of the system. Let $x$ specify the horizontal direction, the potential function can be written as $U(x)=U_{0}[\Theta(x-L / 2+w / 2)-\Theta(x-L / 2-w / 2)] / 2$, where $\Theta$ is the Heaviside step function. The following parameters were used [136] (somewhat arbitrarily): $U_{0}=0.766 t$ and $w=2.5 a$.

\section{Calculation of resonant tunneling rate in graphene systems described by the Hubbard Hamiltonian}

In Sec. VIB 1, a method to calculate the resonant tunneling rate in the single-particle framework is described, based on the time evolution of a special type of initial state localized entirely on one side of the barrier, which is constructed from each eigenstate. When many-body interactions are included, the method needs to be modified. Especially, the interactions can effectively 
be represented by a mean-field potential $U_{H}\left\langle n_{i, \bar{\sigma}}\right\rangle$ for an electron with spin quantum number $\sigma$. With respect to tunneling, there is then an extra potential that is different for spin up and spin down electrons. This extra potential breaks the mirror symmetry and induces localized states with diminishing tunneling. As a result, an additional quantity, the tunneling probability, together with the tunneling rate, is needed to fully describe the tunneling phenomena in relativistic many-body quantum systems.

The method can be described, as follows. The mean-field Hamiltonian is given in Sec. II A 3, i.e.,

$$
\hat{H}_{M F}=-t \sum_{\langle i, j\rangle, \sigma} c_{i, \sigma}^{\dagger} c_{j, \sigma}+U(x, y) \sum_{i, \sigma} c_{i, \sigma}^{\dagger} c_{i, \sigma}+U_{H} \sum_{i, \sigma}\left\langle n_{i, \bar{\sigma}}\right\rangle n_{i, \sigma} .
$$

where $U(x, y)$ characterizes the potential barrier with $U(x, y)=U_{0}$ in the barrier region and zero otherwise, $U_{H}$ is a parameter characterizing the Coulomb interaction in the mean-field Hubbard Hamiltonian. Firstly, one solves the eigenenergy values and eigenstates $\left\{E_{n \sigma},|n\rangle_{\sigma}\right\}$. An arbitrary state $|\psi\rangle_{\sigma}$ can then be written as a linear combination of the eigenstates: $|\psi\rangle_{\sigma}=\sum_{n} a_{n}|n\rangle_{\sigma}$, where $a_{n}$ denotes a set of normalized coefficients. Secondly, for each eigenenergy value $E_{n \sigma}$ and its associated eigenstate, one defines a new wavefunction: $\bar{\psi}_{n, \sigma}(\boldsymbol{r})$, such that the corresponding probability is concentrated entirely on one side of the barrier, say, the left side:

$$
\bar{\psi}_{n, \sigma}(x, y)= \begin{cases}C|n\rangle_{\sigma}, & x \leq(L-w) / 2 \\ 0, & \text { otherwise }\end{cases}
$$

where $C$ is a normalization constant. This new, asymmetrical wavefunction can be expanded in the original base of eigenstates: $|\bar{\psi}\rangle_{n, \sigma}=\sum_{k} b_{k}|k\rangle_{\sigma}$, where $b_{k}$ is a set of expansion coefficients given by $b_{k}=\langle k \mid \bar{\psi}\rangle_{\sigma}$. The time evolution of the wavefunction $|\bar{\psi}\rangle_{n, \sigma}$ is then given by

$$
|\bar{\psi}(t)\rangle_{n, \sigma}=\sum_{k} b_{k} e^{-i E_{k} t / \hbar}|k\rangle_{\sigma}
$$

In general, $b_{n}$ is significantly larger than other coefficients. Depending on the original state, there can be two, three, or a few coefficients that are well separated from the rest of the coefficients that are negligible. As a result, the summation of Eq. (216) can effectively be evaluated using a small number (usually tens) of states with appreciable coefficients.

From Eq. (216), one can write the left-well probability $P_{\sigma}^{L}(t)$ as

$$
P_{\sigma}^{L}(t)=\langle\bar{\psi}(t) \mid \bar{\psi}(t)\rangle_{n, \sigma}^{L}=\sum_{k, k^{\prime}=1}^{N} b_{k} b_{k^{\prime}}\left\langle k \mid k^{\prime}\right\rangle_{\sigma}^{L} e^{-i\left(E_{k^{\prime}}-E_{k}\right) t},
$$

where the upper index $L$ indicates integration over the left well only and $b_{k}$ is real. Since $|\psi(t)\rangle_{n, \sigma}$ can be approximated by a few terms, the sum in Eq. (217) can be approximated by a few terms as well. From direct numerical calculation, it was found [136] that most of the states can be approximated by either two or three eigenstates, with few exceptions.

Suppose initially one chooses a state entirely in the left well: $P^{L}(t=0)=\langle\bar{\psi}(0) \mid \bar{\psi}(0)\rangle_{\sigma}^{L}=$ 1. For $t>0$, the tunneling process begins so that the probability $P^{L}(t)$ decreases with time and reaches its first minimum value $P_{\min }^{L}$ at time $t=\Delta T$. The tunneling rate is conveniently determined by [134] $R=1 / \Delta T$, where the Planck constant has been normalized to unity: $\hbar=1$. Defining $\Delta P=1-P_{\min }^{L}$, one has that $\Delta P$ is the portion that tunnels to the right side of the barrier. The rate $R$ and tunneling probability $\Delta P$ characterize the tunneling process completely. 
Similarly, one can choose an initial state that is localized in the right-hand side of the barrier: $P^{R}(t=0)=\langle\bar{\psi}(0) \mid \bar{\psi}(0)\rangle_{\sigma}^{R}=1$, examine the time evaluation $P^{R}(t)$, and determine the tunneling rate accordingly. Due to symmetry, one has $P_{\sigma}^{L}=P_{\bar{\sigma}}^{R}$, so it is necessary to focus on the tunneling from the left side for spin-up and spin-down states only to obtain a complete picture of the relativistic quantum tunneling dynamics.

\section{Polarization of spin wavefunctions associated with confined states}

To gain intuition, it is useful to study confined states in the absence of any potential barrier for the rectangular geometry. Representative results are shown in Fig. 61(a), where the profiles of the probability density in the horizontal direction for electrons with different spins are presented. It can be seen that the confined states are not polarized, i.e., the spin-up and spin-down wavefunctions are nearly identical in their spatial distributions in the entire domain. The small difference between the spin-specific wavefunctions diminishes for $E \rightarrow t$, where $t$ is the hopping energy between two neighboring atoms.

Consider now the case where there is a narrow potential barrier at the center of the rectangular graphene flake. A surprising phenomenon is that, due to a combined effect of electron-electron interactions and the potential barrier, there are eigenstates in which the spin-up and spin-down electrons become strongly polarized. For example, for the case shown in Fig. 61(b), the spindown (up) electrons tend to focus on the left (right) side of the barrier only. While the case shown in Fig. 61(b) corresponds to wavevector $k_{x} \approx 2 \pi / L$, other polarized states can be found for $k_{x} \approx \pi n / L(n=4,6, \cdots)$. This polarization phenomenon occurs only for the original eigenstates of even parity $(n=2,4,6, \cdots)$ in the absence of the potential barrier. For eigenstates of odd parity $(n=1,3,5, \cdots)$ originally, introduction of a potential barrier does not generate spin polarization.

The polarized states appear in pairs. For example, if there is a polarized state in which the spinup electrons concentrate on the left quantum well (spin-down electrons on the right well), there will be a corresponding polarized state that somewhat mimics a reflected version of the original state, e.g., represented by a wavefunction for which the spin-up electrons concentrate on the right well. The energy difference between the paired states is typically small. Figure 62(a) shows the energy levels of the polarized-state pairs. The red and blue bars with arrow (indicating spins) located in the left or right side denote that the electrons are mainly polarized in the left or right well, respectively. For the specific initial condition used, the average local spin density is positive and negative at the right and left boundary, respectively. Because of the repulsive Coulomb interaction between the electrons, the polarized state with spin-up electrons residing in the right well has a lower energy, while its counterpart has a higher energy. Typical polarized states are shown in Figs. 62(b-c). The accumulation of edge states contributes to the polarized average spin density (magnetic moments) of the graphene sheet. When polarization of the confined states emerges, the polarized magnetic moments remain approximately the same. The reason is that, for each pair, the magnetic moments of the polarized states nearly cancel each other.

The basic physical mechanism for the emergence of the localized spin-polarized states in graphene tunneling system can be understood through the solutions of the Dirac equation governing the spinor wavefunction $\boldsymbol{\psi}=\left[\psi_{A}, \psi_{B}\right]$ corresponding to the pseudospins. The potential term $V$ in the Hamiltonian represents the mean-field potential due to a combined effect of the barrier potential and the edge potential caused by the intrinsic magnetic moments of electron spin. The Coulomb repulsive interaction usually leads to anti-ferromagnetic steady states (mostly edge 

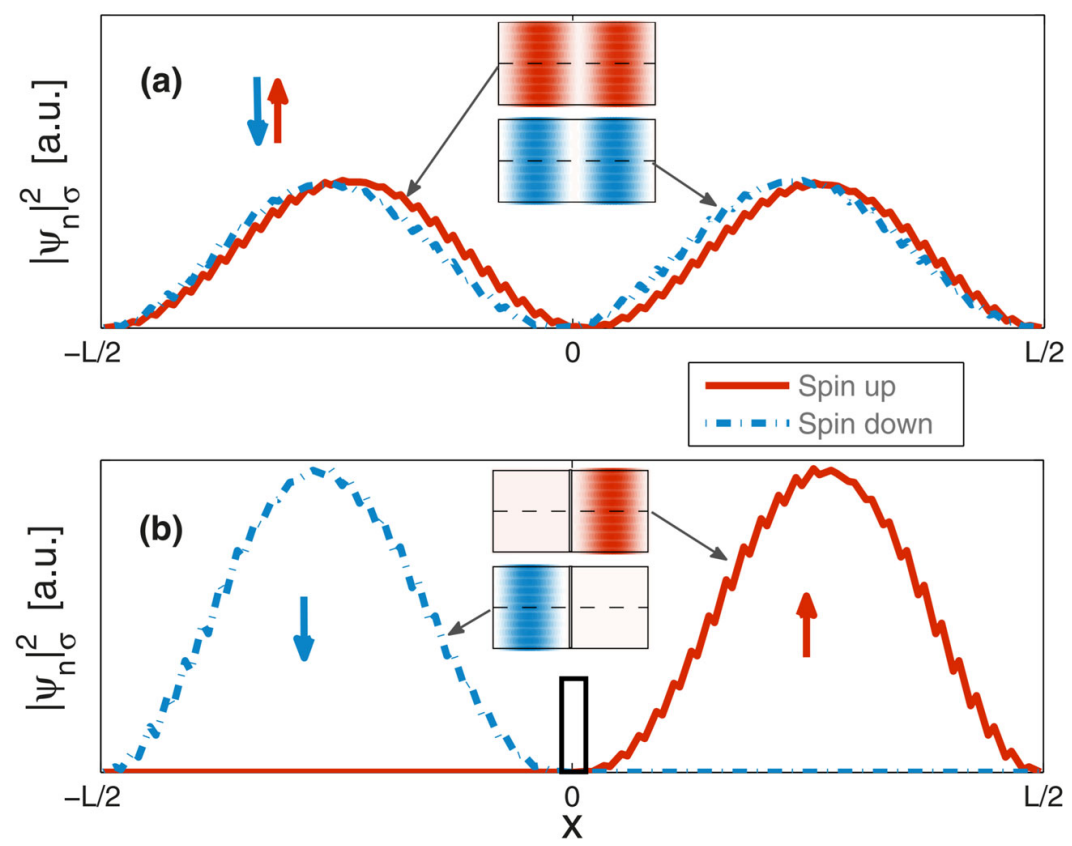

FIG. 61. Emergence of polarization of spin wavefunctions associated with confined states. Representative eigenstates associated with spin-up (red/solid curves) and spin-down (blue/dashed curves) electrons: (a) probability density profile of the 5849'th eigenstate at $y=D / 2$ without a potential barrier, where it extends in both potential wells, (b) probability density profile of the 5860 'th eigenstate at $y=D / 2$ with a potential barrier of height $U_{0}=0.766 t$ and width $w=2.5 a$ at $x=0$ (represented by the gray rectangle). In (b), there is spin polarization, i.e., spin-up electrons reside in the right well and spin-down electrons reside in the left well. The corresponding eigenenergies are $E_{5849} \approx 0.6819 t$ for (a) and $E_{5860} \approx 0.6924 t$ for (b). The insets in both panels show the corresponding LDS patterns in the entire domain. Note that the wavefunctions have a zigzag appearance because they are plotted for both graphene sublattices ( $A$ and $B$ ). A plot of the wavefunction on one sublattice would appear more smooth. From Ref. [136] with permission.

states), with net spin-up electrons residing on the zigzag boundary on one side, while spin-down electrons residing on the other zigzag boundary. These steady states can in turn be regarded as an effective potential that breaks the left-right reflection symmetry and the symmetry breaking can be subdued as a shift of the system. When a potential barrier is applied, it introduces into the system a natural reference point that makes this shift unlikely, yielding spin polarized confined states. These states often appear in pairs, so their magnetic moments cancel each other.

\section{Tunneling rate and tunneling probability of spin-polarized states}

Numerical computation revealed a striking phenomenon [136]: when electron-electron interactions are present, the tunneling probability $\Delta P$, the portion of the state that can tunnel into the other side of the barrier, can be extremely small, e.g., $\Delta P \sim 10^{-4}$, as shown in Fig. 63(a). This should be contrasted to the case of single-electron tunneling, where the value of $\Delta P$ is on the order of unity, as shown in Fig. 63(b) where $\Delta P$ is typically larger than 0.75 . Corresponding to the extremely small values of $\Delta P$, the tunneling rate $1 / \Delta T$ that characterizes the "speed" of tunneling also assumes extremely small values. The reason that $\Delta P$ can be so small for electrons 

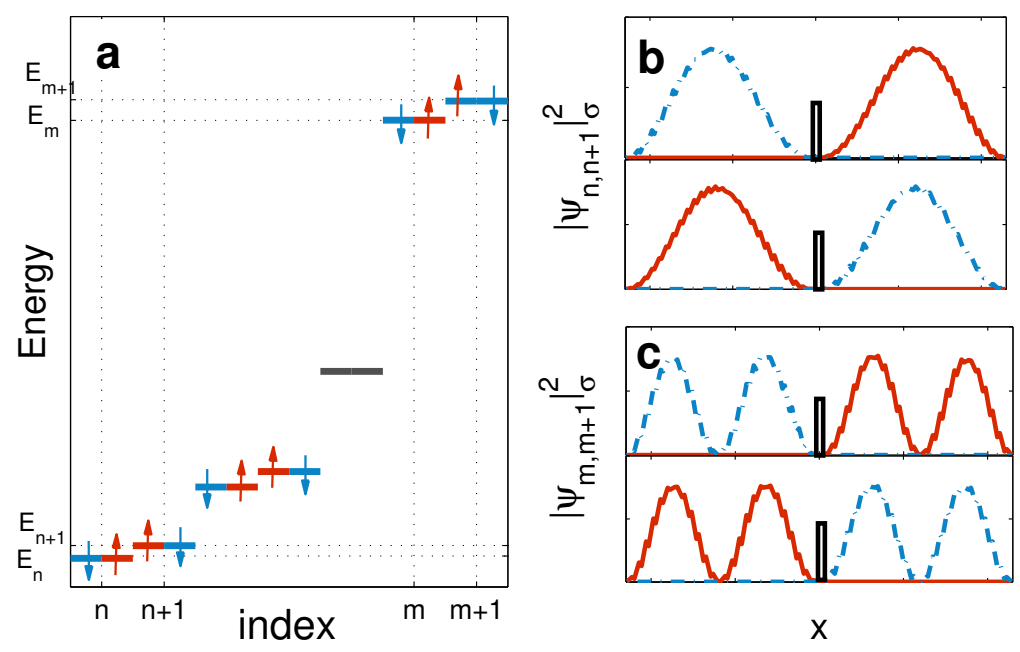

FIG. 62. Emergence of pairwise polarized states. (a) Eigenenergy levels versus the eigenstate index. The red and blue bars with up and down arrows, respectively, represent a pair of polarized states $(n=5760$ and $m=5765$ ), where the corresponding eigenenergies are $E_{n}=0.6203 t, E_{n+1}=0.6204 t, E_{m}=0.6235 t$ and $E_{m+1}=0.6237 t$. The black bar without any arrow corresponds to a unpolarized state. (b, c) Probability densities for the polarized state pairs $(n, n+1)$ and $(m, m+1)$, respectively. Note that the energy difference for each pair is quite small. From Ref. [136] with permission.

with Coulomb interactions lies in the emergence of the spin-polarized states. In particular, in a single-electron tunneling system, because of the left-right reflection symmetry, the eigenfunction also has an even or odd symmetry, and the probabilities for the electron to reside in the left and right wells are equal. When renormalizing the left part of the wavefunction for the situation where the electron initially is located in the left well, the symmetry stipulates that the electron will eventually tunnel to the right side as there is no mechanism to contain it only within the left well. However, for the many-body case where electron-electron interactions are present, the graphene zigzag edge at the opposite boundaries bear different magnetic moments, positive on one side and negative on the other side. Spin-up and spin-down electrons will then "feel" different potentials at the boundaries, leading to the left-right reflection symmetry breaking and, consequently, to the emergence of the spin-polarized states. For example, consider an eigenstate associated with spinup electrons residing mostly in the left well. Because it is an eigenstate, the time evolution of the probability in the left well, $P^{L}$, will be a constant and approximately equal to one. That is, it is a spin polarized and localized state. It can then be deduced that, for spin-up electrons in the left well initially, the associated state will have a significant component in the spin-polarized states, with a high probability $P^{L}$ in the left well at all times, resulting in an extremely small value of $\Delta P$. For comparison, the single-electron tunneling rate was computed [136], as shown in Fig. 63(b). In this case, while the tunneling rate can be small, the tunneling probability $\Delta P$ is generally large, e.g., $\Delta P>0.75$. In addition to the strongly localized states, there are also states that are less localized, resulting in relatively larger values of $\Delta P$. These states can be either spin polarized or unpolarized.

An examination of Fig. 63(a) reveals that the data points can be categorized into three classes, depending on the patterns of their distribution in the $(1 / \Delta T, \Delta P)$ plane. The different tunneling 

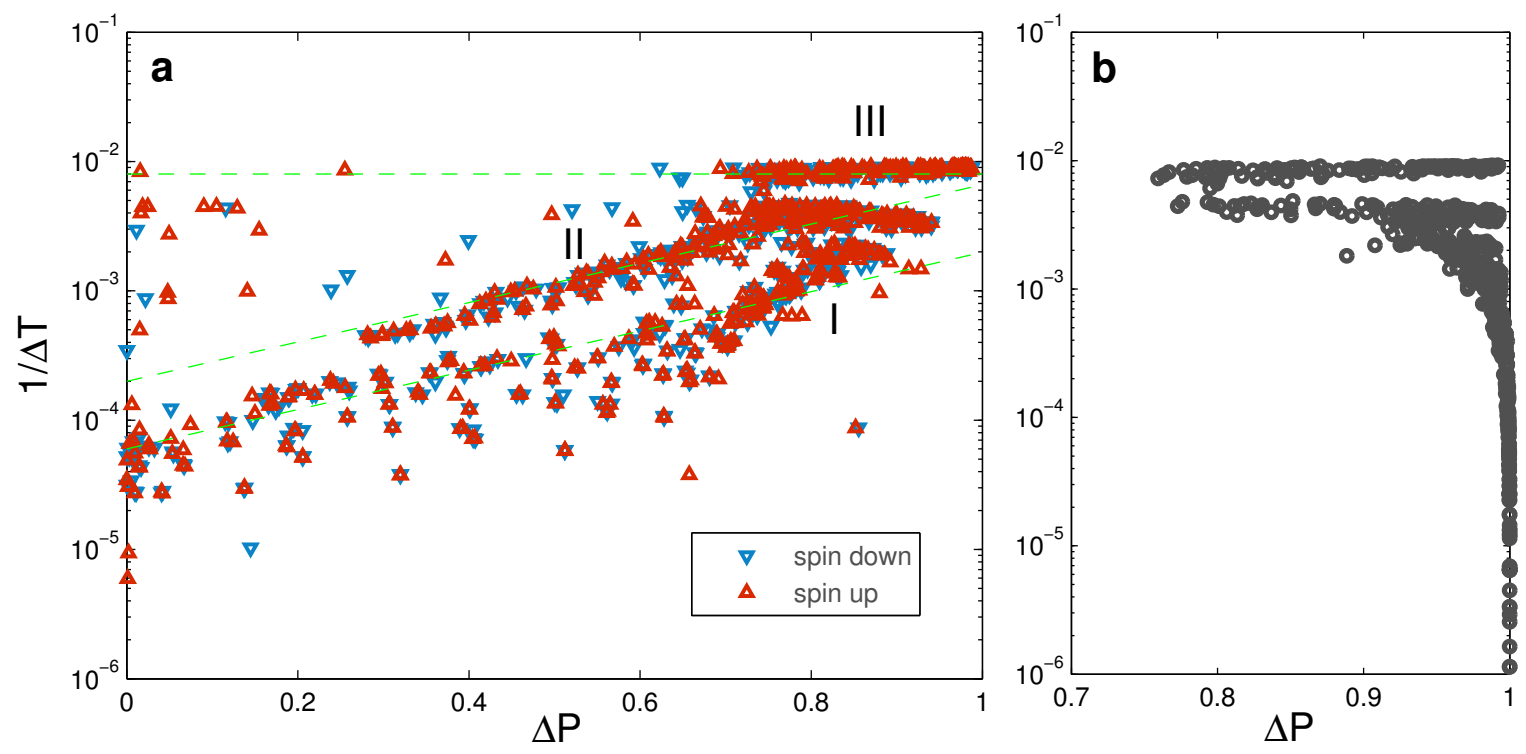

FIG. 63. Behavior of tunneling rate with classically integrable dynamics. Tunneling rate $1 / \Delta T$ versus $\Delta P$ for the rectangular graphene billiard (a) Hubbard model with electron-electron interactions and (b) a single electron. From Ref. [136] with permission.

behaviors correspond to distinct time evolutions of the probability $P^{L}(t)$ for electrons in the left well. In particular, for classes I and II in Fig. 63(a), $P_{L}(t)$ is a cosine function, while for class III, $P_{L}(t)$ is a zigzag curve.

\section{Understanding of the emergence of polarized states through mean-field theory}

In the system described by the mean-field Hubbard Hamiltonian, the states of electrons of spin $\sigma$ are affected by the mean-field electron density of the opposite spin $\left\langle n_{i, \bar{\sigma}}\right\rangle$. This density has appreciable values only at the zigzag edges and it is in fact exponentially small inside the domain [Fig. 64(a)]. As a result, effectively the electron-electron interaction can be described by the following square potential function $\varepsilon_{\sigma} M(x)$ [Fig. 64(b)]:

$$
M(x)= \begin{cases}+M_{0}, & x<\Delta l \\ -M_{0}, & x>L-\Delta l \\ 0, & \text { otherwise }\end{cases}
$$

where $\varepsilon_{\sigma}=\varepsilon_{\uparrow(\downarrow)}=+1(-1)$ for spin up (down), $M_{0}$ is the effective value of $\left(U_{H}\left\langle n_{\sigma(\bar{\sigma})}\right\rangle\right)$ close to the zigzag boundary, and $\Delta l \sim a$ is the width of the effective potential. Note that the effective potentials felt by the spin-up and spin-down electrons are opposite to each other. The overall potential for spin $\sigma$ electrons can thus be expressed as $\widetilde{U}_{\sigma}=U(x)+\varepsilon_{\sigma} M(x)$, and the mean-field Dirac Hamiltonian near the Dirac point $\boldsymbol{K}\left(\boldsymbol{K}^{\prime}\right)$ is given by

$$
\hat{H}_{\boldsymbol{K}\left(\boldsymbol{K}^{\prime}\right), \sigma}=v_{F}\left(\begin{array}{cc}
\widetilde{U}_{\sigma} & -i \partial_{x} \mp \partial_{y} \\
-i \partial_{x} \pm \partial_{y} & \widetilde{U}_{\sigma}
\end{array}\right)
$$




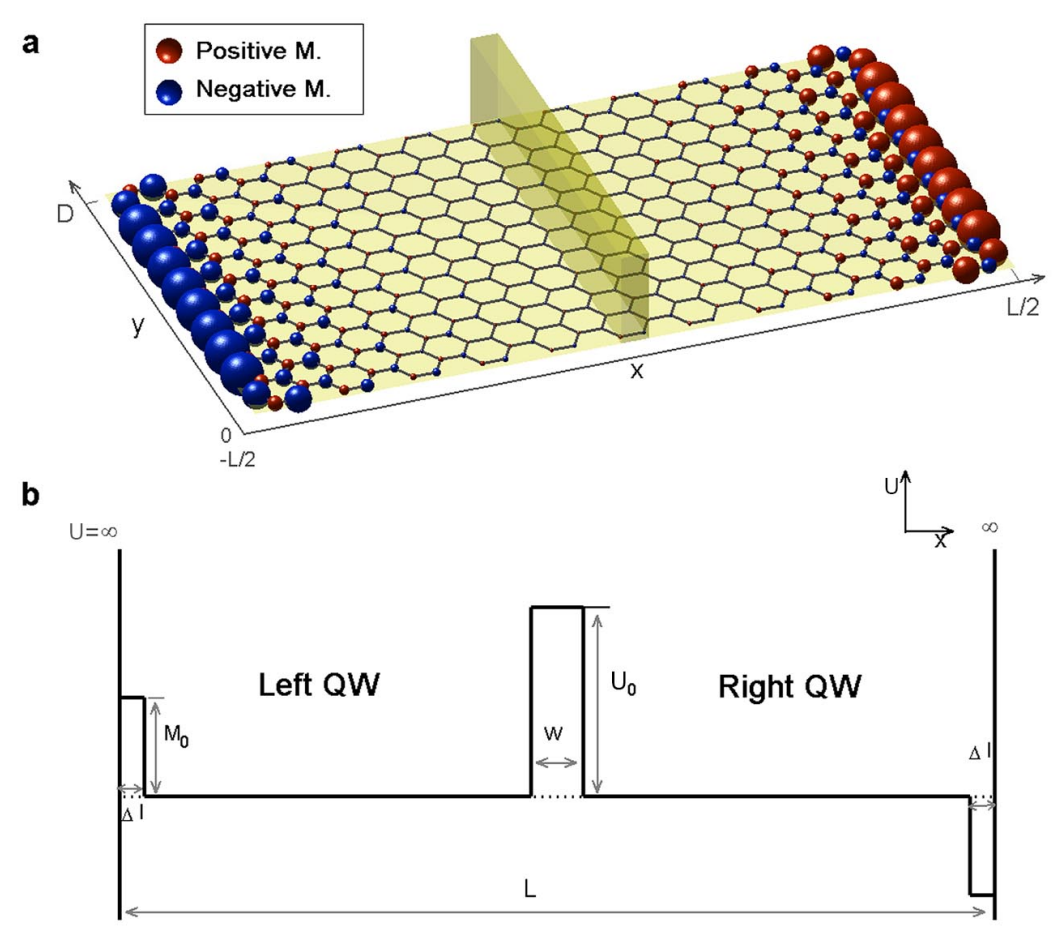

FIG. 64. System setup for mean-field analysis of the emergence of polarized states. (a) Illustration of a rectangular graphene domain of length $L$ and width $D$ with a potential barrier in the middle. The zigzag and armchair edges are along the $y$ - and $x$-axis, respectively. The filled red (blue) circles at the right (left) zigzag boundaries denote the positive (negative) magnetic moments, the radii of which represent the strength of spin density. (b) The effective potential profile $\widetilde{U}_{\uparrow}(x)$ at the position $y=D / 2$ for spinup electrons, where the positive and negative potentials near the boundaries represent the strength of the respective spin density, which approaches the value of $M_{0}\left(-M_{0}\right)$ at the left (right) zigzag edges in (a). The effective potential for spin-down electrons is reversed at the boundaries as compared with that for spin-up electrons. From Ref. [136] with permission.

It is convenient to use small letters $\epsilon, \widetilde{u}$ and $m$ to denote the energy $E$, potential $\widetilde{U}$, and $M$ divided by $\hbar v_{F}$. Note that, the Hamiltonian with the effective potential $\varepsilon_{\sigma} M(x)$ no longer has the left-right reflection symmetry. However, it has the combined symmetry of simultaneous reflection and spin interchange. It is thus only necessary to consider spin-up electrons, because the states of spindown electrons can be obtained straightforwardly by the symmetry operation: $\psi_{\downarrow}(x)=\psi_{\uparrow}(-x)$.

For the $\boldsymbol{K}\left(\boldsymbol{K}^{\prime}\right)$ valley, the bulk eigenstate of the Dirac Hamiltonian Eq. (219) is given by [450]

$$
\left[\psi_{A}^{(\prime)}, \psi_{B}^{(\prime)}\right]^{T}=e^{i \boldsymbol{k} \cdot \boldsymbol{r}}\left[1, \mp e^{ \pm i \phi}\right]^{T}
$$

associated with the energy $E= \pm \hbar v_{F}|\boldsymbol{k}|$, or $\epsilon= \pm|\boldsymbol{k}|$, where $\phi=\tan ^{-1} k_{y} / k_{x}$. Under the influence of the total potential $\widetilde{U}$, the wavevector in the $x$-direction becomes

$$
k_{x}=\sqrt{(\epsilon-\widetilde{u})^{2}-k_{y}^{2}} .
$$

The solutions of the Dirac equations for the armchair or zigzag graphene nanoribbons can be found in Refs. [105, 292]. For the armchair boundaries, the wavevector $k_{y}$ is related with the 
length $D$ in the $y$ direction by $k_{y} \longrightarrow k_{n}=n_{y} \pi / D-4 \pi / 3 a_{0}$, where $n_{y}=0, \pm 1, \pm 2, \cdots$. For an armchair nanoribbon, $k_{n}$ is thus independent of the potential $\widetilde{U}$ and $k_{x}$, and the wavefunction in the $y$-direction can be separated as $\left[\psi_{A}, \psi_{B}\right]^{T}=e^{i k_{n} y}\left[\chi_{A}, \chi_{B}\right]^{T}$, where

$$
\left(\begin{array}{c}
\chi_{A} \\
\chi_{B}
\end{array}\right)=\left(\begin{array}{c}
\frac{1}{\epsilon_{\gamma}}\left[a_{\gamma}\left(k_{n}-q\right) e^{q x}+b_{\gamma}\left(k_{n}+q\right) e^{-q x}\right] \\
a_{\gamma} e^{q x}+b_{\gamma} e^{-q x}
\end{array}\right) .
$$

The coefficients $a_{\gamma}$ and $b_{\gamma}$ in Eq. (220) represent the amplitudes of the wavefunction in the region $\gamma$ and $\epsilon_{\gamma}=\epsilon-\widetilde{u}$ is the relative energy, where $\gamma=L, R, C$ stand for the left, the right and the central barrier regions, respectively. For confined and edge states, we have $q=k_{x}$ and $q=i k_{x}$, respectively.

For zigzag graphene ribbons, the wave vector $k_{x}$ parallel to the armchair edges couples with $k_{n}$ if the potential $\widetilde{u}$ is equal to zero or a constant. In this case, the relationship between the two wavevectors in two orthogonal directions is given by [292]

$$
\left(k_{n}-q\right) /\left(k_{n}+q\right)=\exp (\mp 2 L q),
$$

for the $\boldsymbol{K}\left(\boldsymbol{K}^{\prime}\right)$ point, respectively. A simplified condition of $k_{x}$ for confined states can be rewritten as [105, 292] $k_{x}= \pm \tan ^{-1}\left(k_{x} / k_{n}\right)$ for the $\boldsymbol{K}\left(\boldsymbol{K}^{\prime}\right)$ valley.

Consider first the solution in the $\boldsymbol{K}$ valley without the central barrier. In this case, $k_{n}$ is determined by the width $D$ of the domain but, due to the effective potential $M(x)$ close to the zigzag boundaries, it is infeasible to obtain an exact expression for $k_{x}$. The width $\Delta l$ of this potential is typically much smaller than the length $L$ of the device and is also smaller than the wavelength in the $x$ direction. The following approximations can then be justified. In particular, one can assume that the ratio between the wave amplitude is a constant: $a_{\gamma} / b_{\gamma}=-1$, and the potential $\tilde{u}$ is also a constant for the whole system, which are valid for the case without the central potential barrier. Within distance $\Delta l$ to the boundaries $x=-L / 2$ and $L / 2$, the wavefunction can then be written as

$$
\left(\begin{array}{l}
\chi_{A} \\
\chi_{B}
\end{array}\right) \approx a_{\gamma}\left(\begin{array}{c}
\frac{1}{\epsilon_{\gamma}}\left[-2 q+2 k_{n} q x\right] \\
q x
\end{array}\right)
$$

by first-order linear approximation. In these regions, the only difference from the case where the effective potential is absent lies in the wavevector or the wavelength, i.e., $k_{x}^{ \pm}=\sqrt{(\epsilon \pm m)-k_{n}^{2}}$. Since, what matters here is only the phase change in the wavefunction, one can assume that the wavevector is unchanged but the length of this potential region is changed, say, from $\Delta l$ to $\Delta \widetilde{l}^{ \pm}$ for the right and left boundaries, respectively. One obtains

$$
\Delta \widetilde{l}^{ \pm}=\left(k_{x} / k_{x}^{ \pm}\right) \Delta l
$$

As a result, the wavevectors $k_{\gamma}$ takes on the same value as for the case without the potential $m$ and the only difference is the change in the effective width, $\Delta l$. In particular, at the left boundary, $\Delta l$ shrinks to $\Delta \widetilde{l}^{-}$and, at the right boundary, $\Delta l$ expands to $\Delta \widetilde{l}^{+}$. This is illustrated in Fig. 65(a) in the insets, which show the comparison between the real wavefunction (black solid curve) and the effective wavefunction (red dashed curve) in the potential regions. Effectively, the system is equivalent to a new system without the potential $m$, but with the boundaries shifted by a displacement $\delta_{1(2)}$, as shown schematically in Fig. 65(a). The effective boundary displacement can be determined as

$$
\delta_{1}=\Delta l-\Delta \widetilde{l}^{-} \text {and } \delta_{2}=\Delta \tilde{l}^{+}-\Delta l \text {. }
$$



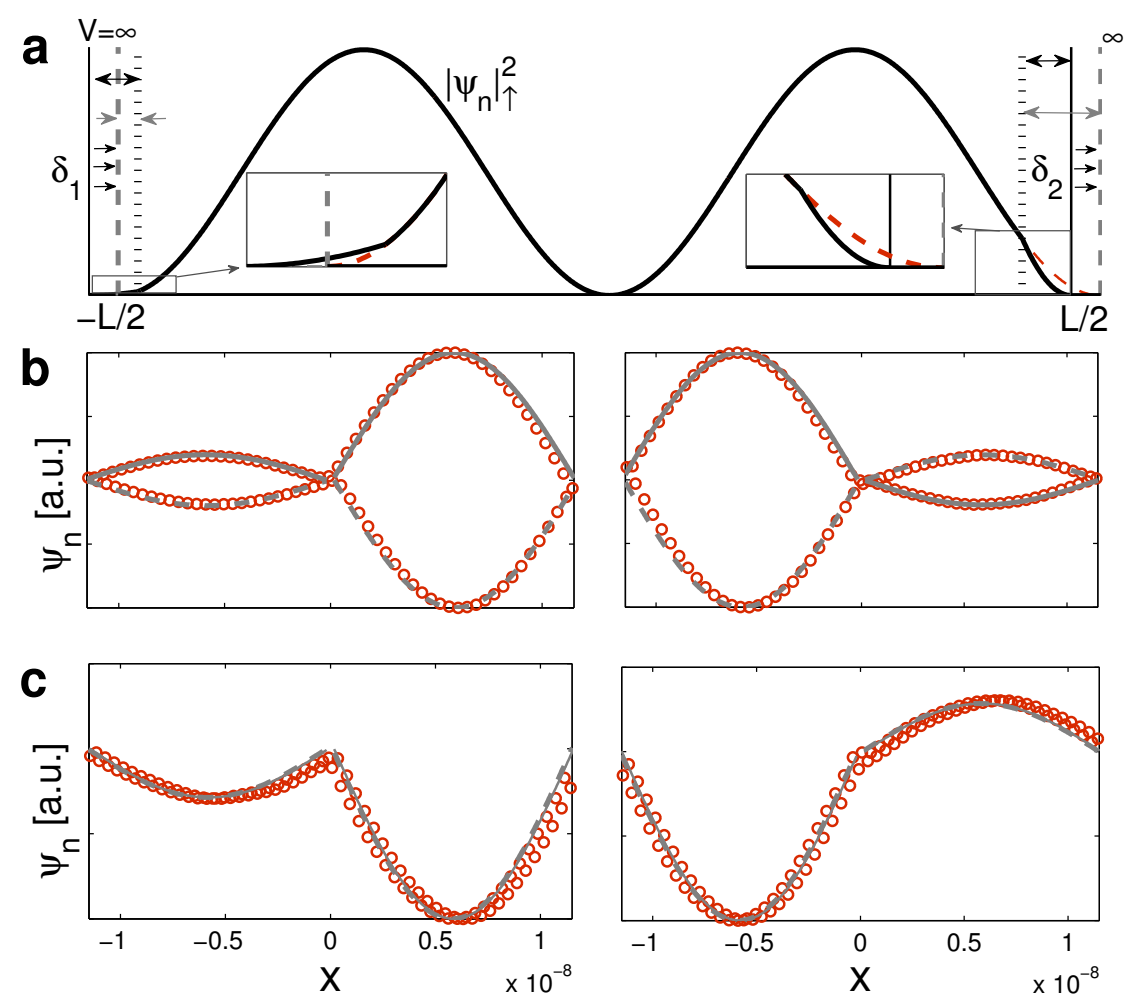

FIG. 65. Understanding of the emergence of the polarized states through construction of equivalent, shifted wavefunctions. (a) Illustration of the shift in the wavefunction caused by the effective potential $M(x)$. The black vertical lines are the actual domain boundaries. The gray lines represent the effective boundaries. The black curves show the actual probability of an evenly confined spin-up state without the potential barrier, and the red curves represent the effective wavefunction after the "shift." (b, c) Antiphase and in-phase wavefunctions for sublattice $A$ and $B$, respectively, where red circles represent the simulation results from the mean-field Hubbard Hamiltonian and the gray curves are predictions from theory [Eqs. $(220,222,223)]$. The parameters are $u_{0}=2.045 / a, \Delta l=2 a, m_{0}=0.2 / a$, and the resulting boundary shift is $\delta=0.032 a$. The wavefunction mode in the $y$-direction is chosen to be $n_{y}=16$ for (b) and $n_{y}=112$ for (c). The numerical results were for the (arbitrarily chosen) states $n=5547$ and 5548 for (b) and $n=5568$ and 5569 for (c). From Ref. [136] with permission.

The physical meaning is that the wavefunctions of spin-up electrons are shifted toward the right (positive axis) by the amount $\delta=\left(\delta_{1}+\delta_{2}\right) / 2$. Because of this relative shift, when a potential barrier is placed in the middle of the domain, the equivalent system with the shift breaks the left-right reflection symmetry, which is key to the emergence of spin polarized states. If energy $\epsilon$ is close to the potential value $m$, the wavevector $k_{x}^{-}$will be purely imaginary and the wavefunction near the left boundary has an exponential form, which differs from a normally propagating wavefunction. But this has little effect on the mean-field based analysis.

It is now feasible to consider the case where a potential barrier is placed along the symmetric line of the domain to understand the emergence of the spin-polarized states. Say one chooses a narrow potential barrier (e.g., of width $w=2.5 a=0.016 L$ ) and places it at $x=0$. From the above analysis, $x=0$ is no longer the center of the equivalent system, as the boundaries have been shifted to the right by the displacement $\delta$ so that the widths of the left- and right-side quantum 
wells become $W_{1} \approx(L-w) / 2-\delta$ and $W_{2} \approx(L-w) / 2+\delta$, respectively. Equivalently, the electron-electron interaction system with a symmetric double well structure can be transformed into a single-electron system with asymmetric double wells.

For an asymmetric double-well system, the standard wavefunctions for graphene [Eq. (220)] satisfy the boundary conditions $\chi_{A(B)}^{L}\left(-L_{1} / 2\right)=\chi_{A(B)}^{R}\left(L_{2} / 2\right)=0$, where $L_{1,2}=2 W_{1,2}+w$. One obtains

$$
b^{L}=-a^{L} e^{-i k_{x} L_{1}} \text { and } b^{R}=-a^{R} \frac{k_{n}^{-}}{k_{n}^{+}} e^{i k_{x} L_{2}},
$$

where $k_{n}^{ \pm}=k_{n} \pm i k_{x}, k_{n}=n_{y} \pi / D-4 \pi / 3 a_{0}$, and $k_{x}$ is to be determined. The continuity conditions at the barrier interfaces are

$$
\begin{gathered}
\chi_{A(B)}^{L}(-w / 2)=\chi_{A(B)}^{C}(-w / 2), \\
\chi_{A(B)}^{R}(w / 2)=\chi_{A(B)}^{C}(w / 2) .
\end{gathered}
$$

When Eq. (222) is applied, the equations of $\boldsymbol{a}=\left[a^{L}, a^{R}, a^{C}, b^{C}\right]^{T}$ can be reorganized as $\boldsymbol{A} \cdot \boldsymbol{a}=$ 0, with $\boldsymbol{A}$ given by

$$
\left(\begin{array}{cccc}
\frac{\epsilon^{\prime}}{\epsilon}\left[k_{n}^{-} e^{-i k_{x} w / 2}-k_{n}^{+} e^{-i k_{x}\left(-w / 2+L_{1}\right)}\right] & 0 & -k_{n}^{-} e^{-i \beta w / 2} & -k_{n}^{+} e^{i \beta w / 2} \\
e^{-i k_{x} w / 2}-e^{-i k_{x}\left(-w / 2+L_{1}\right)} & 0 & -e^{-i \beta w / 2} & -e^{i \beta w / 2} \\
0 & \frac{\epsilon^{\prime}}{\epsilon}\left[k_{n}^{-} e^{i k_{x} w / 2}-k_{n}^{-} e^{-i k_{x}\left(w / 2-L_{2}\right)}\right] & -k_{n}^{-} e^{i \beta w / 2} & -k_{n}^{+} e^{-i \beta w / 2} \\
0 & e^{i k_{x} w / 2}-\frac{k_{n}^{-}}{k_{n}^{+}} e^{-i k_{x}\left(w / 2-L_{2}\right)} & -e^{i \beta w / 2} & -e^{-i \beta w / 2}
\end{array}\right)
$$

where $\beta=\sqrt{k_{n}^{2}-\epsilon^{\prime 2}}, \epsilon^{\prime}=u_{0}-\epsilon$, and $u_{0}=U_{0} / \hbar v_{F}$ is the barrier height. In order to have non-trivial solutions of $\boldsymbol{a}$, the matrix $\boldsymbol{A}$ should satisfy the condition $\operatorname{det}|\boldsymbol{A}|=0$, which in turn solves the only unknown parameter $k_{x}$. In general, a pair of solutions can be found near $2 n \pi / L$, where $n$ is an integer corresponding to the mode number in each quantum well. The final step in the analysis is to obtain the coefficients $\boldsymbol{a}$. Solving for $a^{L} / a^{R}$, one obtains their relative values as

$$
\begin{aligned}
& a^{L}=\frac{\epsilon^{\prime}}{\epsilon} k_{n}^{-}\left[1-e^{-i k_{x}\left(w-L_{2}\right)}\right]-\left(k_{n}+\beta\right)\left[1-\frac{k_{n}^{-}}{k_{n}^{+}} e^{-i k_{x}\left(w-L_{2}\right)}\right] \\
& a^{R}=e^{\left(i k_{x}+\beta\right) w}\left\{\frac{\epsilon^{\prime}}{\epsilon} k_{n}^{-}\left[1-\frac{k_{n}^{+}}{k_{n}^{-}} e^{i k_{x}\left(w-L_{1}\right)}\right]-\left(k_{n}+\beta\right)\left[1-e^{i k_{x}\left(w-L_{1}\right)}\right]\right\} .
\end{aligned}
$$

The relative values of $b^{L, R}$ can be obtained from Eq. (222). With these coefficients, the wavefunction in the $x$-direction can be obtained from Eq. (220).

These theoretical predictions can be compared with numerical results from the mean-field Hubbard Hamiltonian for spin-up wavefunctions, as shown in Fig. 65(b-c). There is a good agreement.

\section{Theory of tunneling rate of spin-polarized states}

Equation (216) gives the time evolution of the wavefunction $|\bar{\psi}\rangle_{n, \sigma}$. The left-well probability $P_{\sigma}^{L}(t)$ is given by Eq. (217). From direct numerical calculation, it was found [136] that most of the states can be approximated by either two or three eigenstates. Particularly, for classes I and II (e.g., Fig. 63), one can approximate $|\bar{\psi}\rangle_{k, \sigma}$ by two eigenstates only:

$$
|\bar{\psi}\rangle_{k, \sigma} \approx b_{k}|k\rangle_{\sigma}+b_{k^{\prime}}\left|k^{\prime}\right\rangle_{\sigma}
$$



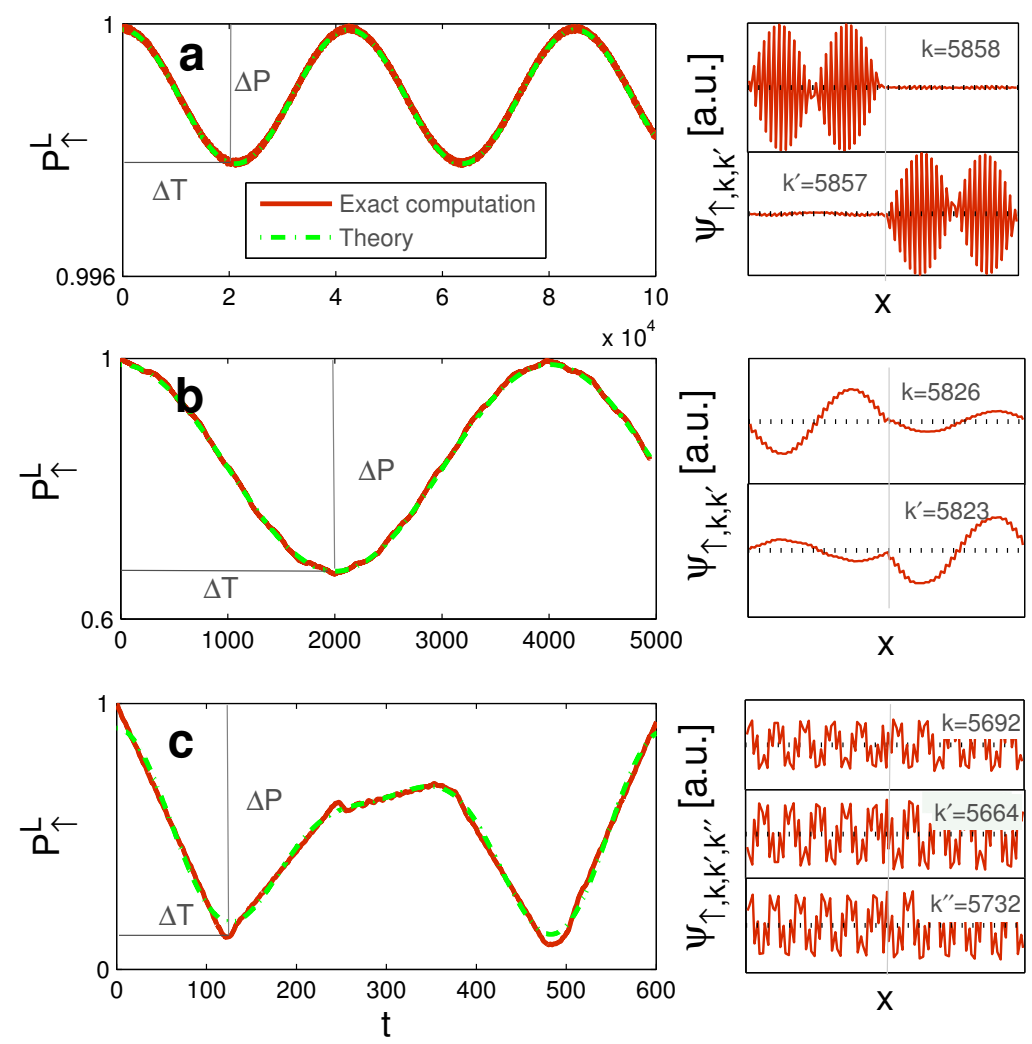

FIG. 66. Tunneling of spin-up states. Shown are the time evolution of $P^{L}(t)$ for spin-up states corresponding to $n=5858$ (a), 5826 (b) and 5692 (c), and their projected states with the corresponding weighting coefficients. The green dashed-dotted curves are fitted from Eqs. (225) (a, b) and (226) (c). From Ref. [136] with permission.

and the expansion coefficients satisfy the relation

$$
b_{k}^{2}+b_{k^{\prime}}^{2} \approx 1
$$

In this case, $|k\rangle_{\sigma}$ and $\left|k^{\prime}\right\rangle_{\sigma}$ are symmetric pairs: $|k(-x)\rangle_{\sigma} \approx\left|k^{\prime}(x)\right\rangle_{\sigma}$, as demonstrated in Fig. 66(a-b). One then has

$$
\langle\bar{\psi} \mid \bar{\psi}\rangle_{k, \sigma}=\langle\bar{\psi} \mid \bar{\psi}\rangle_{k, \sigma}^{L}=1 \approx b_{k}\langle\bar{\psi} \mid k\rangle_{\sigma}^{L}+b_{k^{\prime}}\left\langle\bar{\psi} \mid k^{\prime}\right\rangle_{\sigma}^{L}
$$

Comparing this equation with Eq. (224), one gets $\langle\bar{\psi} \mid k\rangle_{\sigma}^{L} \approx b_{k}$, and $\left\langle\bar{\psi} \mid k^{\prime}\right\rangle_{\sigma}^{L} \approx b_{k^{\prime}}$. Note that $\langle\bar{\psi} \mid k\rangle_{\sigma}^{L} \approx b_{k}$ is equivalent to $|k\rangle_{\sigma}^{L}=b_{k}|\bar{\psi}\rangle_{\sigma}^{L}$, as the former can be obtained by multiplying $\left\langle\left.\bar{\psi}\right|_{\sigma} ^{L}\right.$ to the latter. Similarly, one has $\left|k^{\prime}\right\rangle_{\sigma}^{L}=b_{k^{\prime}}|\bar{\psi}\rangle_{\sigma}^{L}$. Substituting these relations back into Eq. (217), one obtains the time evolution of left-side probability as

$$
\begin{aligned}
P_{\sigma}^{L}(t) & \approx b_{k}^{2}\langle k \mid k\rangle_{\sigma}^{L}+b_{k^{\prime}}^{2}\left\langle k^{\prime} \mid k^{\prime}\right\rangle_{\sigma}^{L}+2 b_{k} b_{k^{\prime}}\left\langle k \mid k^{\prime}\right\rangle_{\sigma}^{L} \cos (\Delta E \cdot t) \\
& =b_{k}^{4}+b_{k^{\prime}}^{4}+2 b_{k}^{2} b_{k^{\prime}}^{2} \cos (\Delta E \cdot t),
\end{aligned}
$$

where $\Delta E=E_{k^{\prime}}-E_{k}$. Note that $\left\langle k^{\prime} \mid k^{\prime}\right\rangle_{\sigma}^{L}$ is not zero because the integration is with respect to the left well only. Thus, the $P_{\sigma}^{L}(t)$ curve is a standard cosine-type function. At $t=0$, one has

$$
P_{\sigma}^{L}=b_{k}^{4}+b_{k^{\prime}}^{4}+2 b_{k}^{2} b_{k^{\prime}}^{2}=\left(b_{k}^{2}+b_{k^{\prime}}^{2}\right)^{2} \approx 1 .
$$


It can be seen that $P_{\sigma}^{L}(t)$ reaches its first minimum at $\Delta T=\pi / \Delta E$, which is

$$
P_{\sigma}^{L}=b_{k}^{4}+b_{k^{\prime}}^{4}-2 b_{k}^{2} b_{k^{\prime}}^{2}=\left(b_{k}^{2}-b_{k^{\prime}}^{2}\right)^{2} .
$$

As a result, the tunneling probability into the right well is given by $\Delta P=4 b_{k}^{2} b_{k^{\prime}}^{2}$.

To validate the approximation used in the analysis, two typical states $(k=5858$ and 5826$)$ were chosen [136], which can be well approximated by two eigenstates, $|k\rangle_{\sigma}$ and $\left|k^{\prime}\right\rangle_{\sigma}$. The quantities $b_{k}$ and $b_{k^{\prime}}$ can then be computed and the approximate result Eq. (225) can be compared with the accurate result Eq. (217) of the time evolution of $P_{L}^{\sigma}(t)$, as shown in Figs. 66(a,b). There is a good agreement.

Both type-I and type-II classes have the cosine time evolution of $P_{\sigma}^{L}$, but they are well separated in the $(1 / \Delta T, \Delta P)$ plane. The main reason lies in the phase difference between the wavefunctions on the sublattices $A$ and $B$, where the phases can have a $\pi$ difference, or they can be the same, as illustrated by the eigen-wavefunctions in Figs. 66(a,b). Due to the phase differences, the energy difference $\Delta E$ (thus the tunneling rate $R$ ) differs as well. For the eigen-wavefunctions with antiphase with respect to $A$ and $B$, the energy difference is considerably smaller than that for the case of identical phases, resulting in the separation as exemplified in Fig. 63(a).

For class III pattern, the quantity $|\bar{\psi}(t)\rangle_{k, \sigma}$ can be approximated by three eigenstates,

$$
|\bar{\psi}\rangle_{k, \sigma} \approx b_{k}|k\rangle_{\sigma}+b_{k^{\prime}}\left|k^{\prime}\right\rangle_{\sigma}+b_{k^{\prime \prime}}\left|k^{\prime \prime}\right\rangle_{\sigma}
$$

where $b_{k^{\prime \prime}}$ is comparable to $b_{k^{\prime}}$, and they are both smaller than $b_{k}$. Normalization condition requires

$$
b_{k}^{2}+b_{k^{\prime}}^{2}+b_{k^{\prime \prime}}^{2} \approx 1
$$

For this class, the states are generally not polarized and they distribute approximately evenly in the left and right wells. One thus has

$$
\langle k \mid k\rangle_{\sigma}^{L} \approx\left\langle k^{\prime} \mid k^{\prime}\right\rangle_{\sigma}^{L} \approx\left\langle k^{\prime \prime} \mid k^{\prime \prime}\right\rangle_{\sigma}^{L} \approx 1 / 2
$$

Substituting this relation into Eq. (217) leads to the time evolution of the left-well probability as

$$
\begin{aligned}
P_{\sigma}^{L}(t) & \approx b_{k}^{2}\langle k \mid k\rangle_{\sigma}^{L}+b_{k^{\prime}}^{2}\left\langle k^{\prime} \mid k^{\prime}\right\rangle_{\sigma}^{L}+b_{k^{\prime \prime}}^{2}\left\langle k^{\prime \prime} \mid k^{\prime \prime}\right\rangle_{\sigma}^{L}+2 b_{k} b_{k^{\prime}}\left\langle k \mid k^{\prime}\right\rangle_{\sigma}^{L} \cos \left(\Delta E^{\prime} \cdot t\right) \\
& +2 b_{k} b_{k^{\prime \prime}}\left\langle k \mid k^{\prime \prime}\right\rangle_{\sigma}^{L} \cos \left(\Delta E^{\prime \prime} \cdot t\right) \\
& \approx 1 / 2+2 b_{k} b_{k^{\prime}}\left\langle k \mid k^{\prime}\right\rangle_{\sigma}^{L} \cos \left(\Delta E^{\prime} \cdot t\right)+2 b_{k} b_{k^{\prime \prime}}\left\langle k \mid k^{\prime \prime}\right\rangle_{\sigma}^{L} \cos \left(\Delta E^{\prime \prime} \cdot t\right),
\end{aligned}
$$

where $\Delta E^{\prime}=E_{k^{\prime}}-E_{k}, \Delta E^{\prime \prime}=E_{k^{\prime \prime}}-E_{k}, b_{k}$ 's, $\left\langle k \mid k^{\prime}\right\rangle_{\sigma}^{L}$, and $\left\langle k \mid k^{\prime \prime}\right\rangle_{\sigma}^{L}$ can be determined numerically. A representative case is shown in Fig. 66(c) with both the accurate result in Eq. (217) and the approximation in Eq. (226). The approximated results agree with those from the exact calculation reasonably well. For more complicated cases that have four and more large coefficients of $b_{k}$, the approximation method remains valid [136].

\section{Regularization of tunneling by chaos}

The understanding of the spin-polarized confined states in the rectangular graphene indicates that many-body interactions are key to the emergence of such states. These states have a significant effect on the quantum tunneling dynamics. Especially, for the rectangular graphene billiard, not 

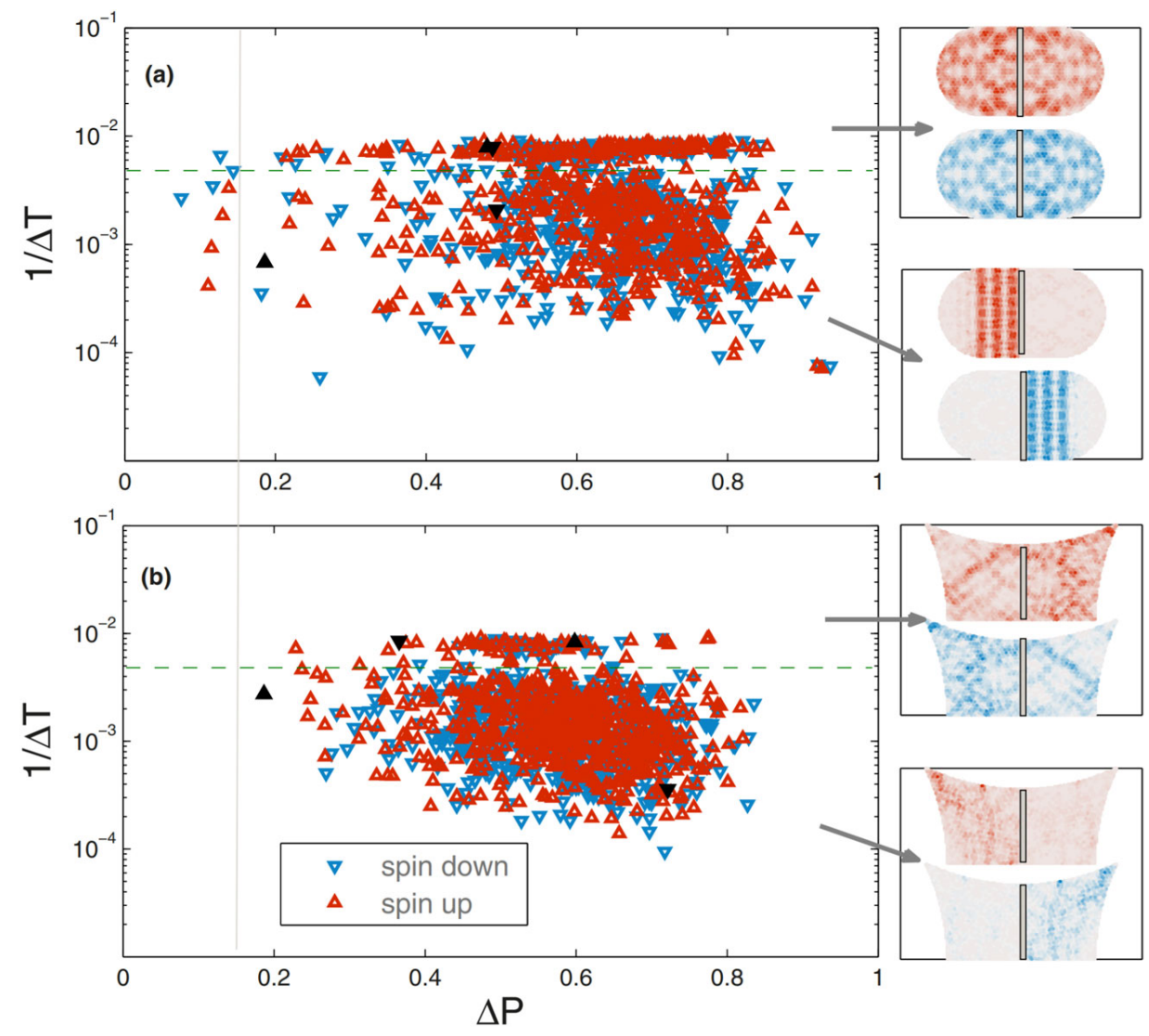

FIG. 67. Behavior of tunneling rate with classically chaotic dynamics. Tunneling rates versus $\Delta P$ for chaotic geometries: (a) stadium and (b) bowtie graphene billiards. The green dashed horizontal line indicates the separation of the two classes of spin polarized (lower) and unpolarized (upper) states. The black solid up- and down-triangles correspond to the typical LDS patterns in each class for spin-up and -down states, respectively. From Ref. [136] with permission.

only can the tunneling rate be negligibly small (corresponding to large values of $\Delta T$ ), but also the tunneling magnitude $\Delta P$ is near zero. While other physical factors such as the potential barrier and the pseudospin degree of freedom of graphene also play a role in the formation of the localized spin-polarized states, many-body interactions are the single most important factor. This is because, without such interactions, the tunneling rate and magnitude can typically be appreciable in graphene systems of the same geometry [134]. Does this mean that, in any realistic graphene system where many-body interactions are inevitably present, tunneling is far less likely? If this were the case, it would be difficult to develop graphene-based tunneling device. It was found [136] that this difficulty can be overcome by taking advantage of classical chaos. In particular, when the geometry of the domain is such that the corresponding classical dynamics are chaotic, both tunneling rate and magnitude can be significantly enhanced, suggesting that chaotic geometries are favorable if fast and sizable tunneling is needed. Since the effective geometrical shape of the domain can be modulated by external means, such as atomic probe [451] or gate voltage, quantum tunneling dynamics in the presence of many-body interactions can be harnessed by using chaos.

Figure 67 shows the tunneling rates for a stadium-shaped and a bowtie-shaped graphene billiard. As compared with the rectangular billiard, for both types of chaotic billiards, the points in 
the plot of the tunneling rates $1 / \Delta T$ versus $\Delta P$ in Fig. 67 are more concentrated in both dimensions. The concentration is more compact for the bowtie billiard (the "more chaotic" domain). Some pronounced features of Fig. 67 are the following.

Firstly, as compared with the rectangular domain, the tunneling rates associated with the chaotic domains are greatly enhanced, e.g., from about $10^{-6}$ in Fig. 63 to about $10^{-4}$ in Fig. 67. Comparing Fig. 67(a) with Fig. 67(b), one sees that overall, the tunneling rates for the "more chaotic" bowtie billiard are even larger.

Secondly, the range of $\Delta P$ is reduced in the chaotic systems in the sense that, as compared with the rectangular domain, the minimum value of $\Delta P$ is larger and its maximum value becomes smaller. For the stadium and bowtie domains, the ranges are from 0.1 to 0.9 and from 0.2 to 0.8 , respectively. A common feature is that the localized states with extremely small values of tunneling rate ceases to exist. Thus, the nearly perfect spin polarized states in the integrable domain have been effectively eliminated by chaos!

Thirdly, for the integrable system, as shown in Fig. 63, the points in the plot of $1 / \Delta T$ versus $\Delta P$ are grouped into three classes. For both types of chaotic domain, as shown in Fig. 67, the points only belong to two classes. The first class is for $1 / \Delta T \sim 10^{-2}$, which is similar to case III for the rectangular domain in that the eigenstates are not polarized but distributed equally between the left and right wells. It might be tempting to regard the lower points in Fig. 67 (especially in the side panels) as corresponding to some sort of spin-polarized states. However, in contrast to the rectangular system in which the states are either antiphase (class I) or having identical phases for $A$ and $B$ atoms (class II), the eigenstates for the chaotic domains do not have such a clear cut as the relative phases assume randomized values between 0 and $\pi$.

It can then be concluded that, when electron-electron interactions are taken into account in a graphene system, chaos can not only regularize the tunneling rate as in situations where such interactions are neglected $[134,135,180]$, but also regularize the polarization and mix the relative phase between the states associated with the two distinct sublattices.

For the mushroom billiard shown in Fig. 60(d) that generates classically nonhyperbolic dynamics, Fig. 68 shows the tunneling rate $1 / \Delta T$ versus $\Delta P$. The points are scattered in three regions, which are separated by the horizontal dashed lines. The middle and lower parts are similar to the two regions for the chaotic systems in Fig. 67. Specifically, the middle and lower parts correspond to unpolarized and polarized states, respectively. The upper region, which is absent from both integrable and chaotic systems, corresponds to the integrable part of the mushroom billiard in the stem region. In Fig. 68, the minimum value of $\Delta P$ is about 0.3 , which is larger than those for both chaotic domains. For the mushroom billiard, the classical periodic orbits generally cross both the left and the right parts, so strong polarized states are less likely to form, leading to relatively larger values of $\Delta P$. These results are quantitatively consistent with those in absence of electron-electron interactions [135].

\section{Remarks on the interplay among chaos, relativistic quantum mechanics, and many-body effects}

The emergence of the spin polarized states is a combined effect of electron-electron interaction, the pseudo-spin freedom of graphene sublattices, and the potential barrier. The localization phenomenon is somewhat similar to the general localization effect in one-dimensional fermion systems, i.e., the addition of an arbitrary weak barrier at a given point effectively freezes the tunneling through the barrier [452], but with a different mechanism. The single quantity to char- 


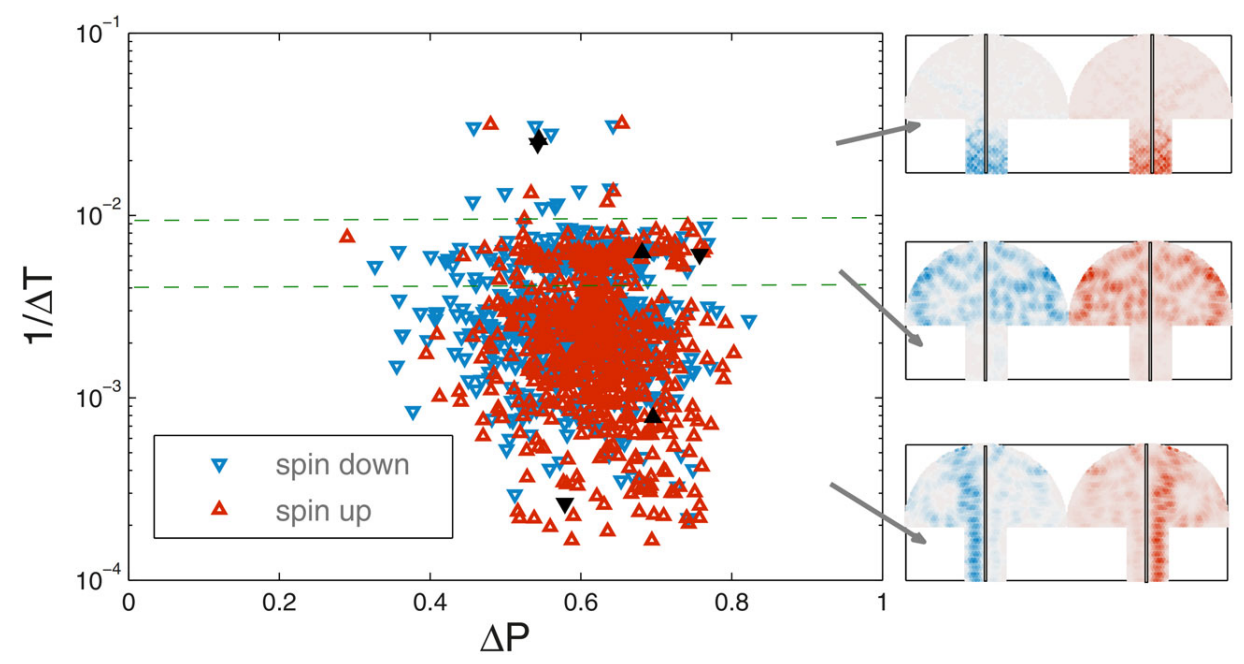

FIG. 68. Behavior of tunneling rate with classically nonhyperbolic dynamics. Tunneling rates versus $\Delta P$ for the nonhyperbolic mushroom billiard. The green dashed horizontal lines separate the data points into three different classes. The middle and lower classes come from the chaotic components. The upper class is originated from the stem of the mushroom billiard. The black solid up- and down-triangles correspond to the LDS patterns for spin-up and spin-down states in the right-side panels, respectively. From Ref. [136] with permission.

acterize quantum tunneling dynamics, namely the tunneling rate, is insufficient for describing the tunneling behavior associated with the localized spin-polarized states. In fact, tunneling strength is also necessary. For the spin-polarized states, not only can the tunneling rate be negligibly small, but also the tunneling strength. For these states, the spin-up and spin-down electrons are separated in space by the potential barrier. As a result, if an electron occupies a spin-up state, it will remain so for all times. This should be contrasted to the case of absence of many-body interactions, where the tunneling strength can typically be much larger, i.e., on the order of one.

When the geometry is changed so that the classical dynamics becomes chaotic, the spreads in both the tunneling rate and strength are greatly suppressed. The states with extremely small tunneling rate and strength are effectively removed. A more chaotic system has a stronger ability to regularize the tunneling dynamics. The surprising result is then that chaos can significantly enhance tunneling in realistic situations where electron-electron interactions are present. This implies that classical chaos is capable of facilitating greatly relativistic quantum tunneling, which is desired when developing nanoscale devices such as graphene-based resonant-tunneling diodes.

The mean-field approach to solving the Hubbard model for graphene systems requires a justification. In Ref. [213], it was indicated that the self-consistent mean-field approach is accurate when the Hubbard interaction strength $U_{H}$ is not too large. For $U_{H}=1.2 t$ used in the study [136], it is well within the regime of validity of the mean-field Hubbard model, i.e., $U_{H} \lesssim 2 t$. In Ref. [215], the dynamical properties of edge state magnetism in graphene systems were investigated. The results of static spin polarization from the mean-field theory were also compared with those from the quantum Monte-Carlo (QMC) approach. A main conclusion of Ref. [215] was that the results from the two approaches agree each other well in terms of the static properties. With respect to the dynamic properties, the agreement holds but for narrow graphene ribbons. In general, the Hubbard model is paradigmatic in that it does capture the electron-electron interactions in graphene 
systems and the self-consistent mean-field approach is effective for analyzing the effects of the interactions. In fact, there was a work [453] investigating the edge spin-polarization for large systems, e.g., $10^{4}$ carbon atoms. It was found that, if the environmental time scale $\tau_{\text {env }}$ is much shorter than $\tau_{q d}$, the system is pushed into the same classical Néel-like state again and again. As a result, the state cannot decay, which is known as the quantum Zeno effect [453]. Furthermore, the analysis in Sec. VID 5 does not require perfect spin polarization at opposite zigzag edges. In the presence of quantum fluctuations, if there are noticeable remnant edge magnetic states, which introduce left-right asymmetry to the spin electrons, the eigenstates will be spin-polarized for the left and the right domains when a central potential barrier is present.

There are several issues associated with experimental realizations. One is the size of the potential barrier. In the simulation [136], the width of barrier is $0.4 \mathrm{~nm}$, which may be too small for a gate potential to be applied, as the current experimental techniques would allow the minimum width of the top gate to be about $10 \mathrm{~nm}$ [454]. Nonetheless, based on the theoretical prediction in Eq. (223), the spin-polarized state is not affected by the width of the central potential barrier: it only depends on the ratio between the barrier width $w$ and device length $L$. This allows an experimental study of the localization phenomenon if one enlarges $w$ and $L$ proportionally so that $w$ is sufficiently large, say, $15 \mathrm{~nm}$. Another consideration is that the barrier in an experimental realization may not be a square potential but with small deformation. This, however, does not present any serious challenge because the spin-polarized states are stable due to the edge magnetic moments. As a result, the polarized states can still exist and similar regularization effects of chaos should persist. 


\section{SUPERPERSISTENT CURRENT IN CHAOTIC DIRAC FERMION SYSTEMS}

\section{A. Persistent currents in quantum systems - an introduction}

A remarkable phenomenon in the quantum world is persistent or permanent currents (PCs) [182], i.e., currents requiring no external voltage with zero resistance. Earlier, PCs were thought to occur only in superconductors. However, more than three decades ago, it was theoretically predicted [182] that such dissipationless currents can emerge in normal metallic or semiconductor ring systems subject to a central AB [183] magnetic flux. In particular, breaking of time-reversal symmetry by the magnetic flux makes the magnitudes of the currents in the clockwise and counterclockwise directions different, generating a net current. From a microscopic point of view, if the ring size is smaller than the elastic scattering length, the electron motion in the entire domain will become ballistic without scattering. If the ring size is larger than the elastic scattering length, the electron's behavior will be diffusive with a current proportional to $1 / \tau_{D}$, where $\tau_{D}$ is the diffusion time around the ring. While the environmental temperature needs to be sufficiently low to reduce inelastic scattering from phonon-electron and/or electron-electron interactions for the currents to be observed [182, 455-457], the metallic material itself remains "normal" (i.e., not superconducting).

Experimentally, PCs have been observed in metallic [184-188] and semiconductor [189-191] rings in the mesoscopic regime. Theoretical efforts were focused on the effects of bulk disorders [455, 458, 459], electron-electron interactions [456, 460, 461], spin-orbital interactions [462, 463], and electromagnetic radiation [464, 465] on PCs, typically studied in the diffusive regime using idealized circular-symmetric rings or cylinders. Rapid advances in nanotechnology made it feasible to fabricate mesoscopic devices with mean free path larger than their sizes at sufficiently low temperatures (the ballistic transport regime) [377]. The AB ring system can thus be modeled as a quantum ballistic billiard in which the particles are scattered at the boundaries of the domain. As a result, the boundary shape becomes highly relevant.

PCs in nonrelativistic quantum systems are vulnerable to material impurities, which fundamentally restricts the phenomenon to systems at or below the mesoscopic scale. Indeed, in real materials, disorders are inevitable, which can dramatically reduce the phase coherent length due to enhanced random scattering. In general, random disorders can remove the energy degeneracies and induce level repulsion, opening energy gaps and destroying the conducting state. As a result, disorders in metallic or semiconductor systems, $1 \mathrm{D}$ or $2 \mathrm{D}$, tend to diminish the persistent currents [193, 195, 466-470]. As the strength of the disorder is increased, the currents decay exponentially to zero $[466,470]$.

In experiments, uncontrollable boundary imperfections are inevitable [198, 471] even when there are no bulk disorders. It is thus of interest to study the effects of boundaries, e.g., those that generate chaos in the classical limit, on PCs. In general, an asymmetric boundary destroys angular momentum conservation and introduces irregular scattering. Theoretical [192-197] and experimental [198] studies showed that, similar to the effects of bulk disorder, symmetry breaking of the boundary can result in gap opening at the degeneracy points of the energy levels, leading to level repulsion and diminishing conduction, a typical manifestation of classical chaos. Energy gap opening can suppress $\mathrm{AB}$ oscillations through pinning of the corresponding states, giving rise to vanishing PCs. Since fully chaotic domains are associated with a strong degree of symmetry breaking, PCs are not expected to arise in such systems [472, 473]. In nonrelativistic quantum 
systems governed by the Schrödinger equation, PCs are thus not robust.

An interesting question was whether, in relativistic quantum systems, PCs can arise and sustain in the presence of symmetry-breaking perturbations. Besides the relevance of this question to fundamental physics, there was an interest in investigating relativistic PCs in Dirac materials systems [474-486] with device implications. The theoretical works on relativistic PCs in graphene, however, had assumed idealized circular-symmetric rings in the ballistic limit. Whether AB oscillations and consequently relativistic PCs can persist in asymmetric rings that exhibit chaos in the classical limit was a fundamental issue. This issue was addressed [137] where it was found that, even in the presence of significant boundary deformations so that the classical dynamics becomes fully chaotic, robust PCs can still occur in relativistic quantum, Dirac fermion systems, henceforth the term superpersistent currents (SPCs) [137]. Another result was that SPCs are generated by circulating states at the domain boundaries, which are effectively chaotic Dirac whispering gallery modes (WGMs) that carry larger angular momenta. While WGMs are common in photonic systems [487-490], its emergence in electronic and laser systems [491-493], especially in relativistic quantum systems, was considered rare and surprising. The insensitivity of Dirac WGMs to boundary deformations may be intuitively understood as a consequence of the zero normal flux boundary condition required for nontrivial, physically meaningful solutions of the Dirac equation.

\section{B. Superpersistent current in chaotic Dirac fermion systems}

\section{A prototypical model of relativistic $A B$ system}

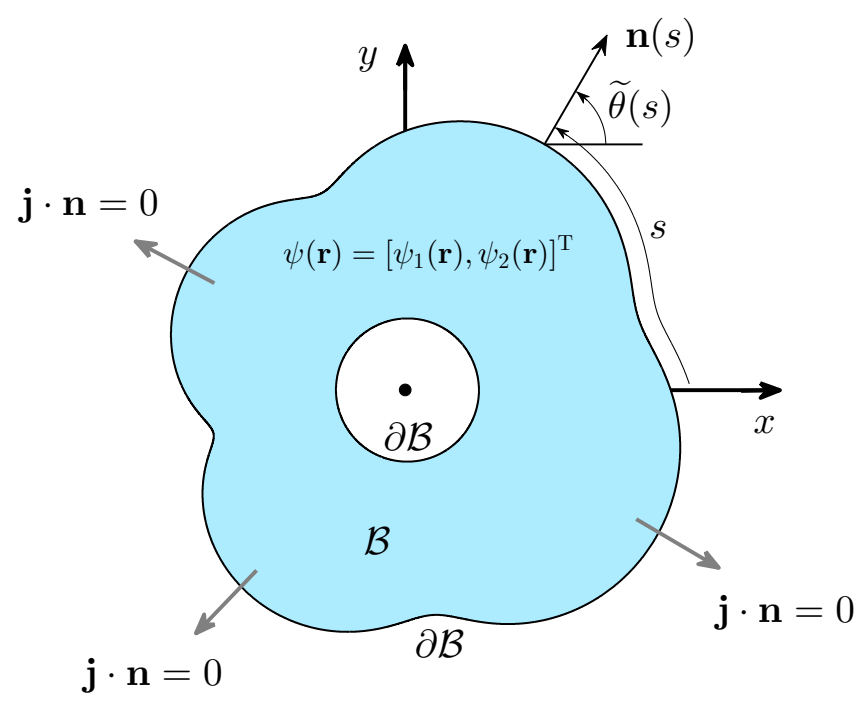

FIG. 69. Illustration of a paradigmatic system for studying persistent currents. Shown is a chaotic ring domain with boundary parameterized by the arc length $s$. For motion of a massless Dirac fermion inside the domain, the boundary condition is of the zero-flux type, i.e., no outward current at any point $s: \boldsymbol{j} \cdot \boldsymbol{n}=0$. From Ref. [137] with permission.

Consider a single massless spin-half particle of charge $-e$ confined by hard walls in a domain $\mathcal{B}$ with a ring topology in the plane $\boldsymbol{r}=(x, y)$, as shown in Fig. 69. Applying a single line of mag- 
netic flux (AB flux) $\Phi$ at its origin and utilizing an infinite mass term outside the domain to model the confinement, one obtains the Hamiltonian in Eq. (11) but with the speed of light $c$ replaced by the Fermi velocity, where the potential $V(r)$ defines the hard wall boundaries. Such boundaries were used in the studies of graphene rings [251], graphene quantum dots [494], and topological insulator quantum dots [341]. The vector potential $\boldsymbol{A}(\boldsymbol{r})$ can be chosen as any vector field satisfying $\boldsymbol{\nabla} \times \boldsymbol{A}(\boldsymbol{r})=\hat{z} \alpha \Phi_{0} \delta(\boldsymbol{r})$, where $\hat{\boldsymbol{z}}$ is the unit vector perpendicular to $\mathcal{B}$ and $\alpha \equiv \Phi / \Phi_{0}$ is the dimensionless quantum flux parameter. The Hamiltonian $\hat{H}$ acting on the two-component spinor wave-function $\psi(\boldsymbol{r})=\left[\psi_{1}, \psi_{2}\right]^{\mathrm{T}}$ has eigenvalue $E$ :

$$
\left[-i \hbar v_{F} \hat{\boldsymbol{\sigma}} \cdot \boldsymbol{D}+V(\boldsymbol{r}) \hat{\sigma}_{z}\right] \psi(\boldsymbol{r})=E \psi(\boldsymbol{r}),
$$

where $\boldsymbol{D}=\boldsymbol{\nabla}+i \boldsymbol{a}$ is a compact notation for the covariant derivative with $\boldsymbol{a} \equiv 2 \pi \boldsymbol{A} / \Phi_{0}$.

In Eq. (228), $V \sigma_{z}$ represents the mass potential that makes the Klein tunneling effect irrelevant, confining a massless Dirac fermion in a finite domain. Such a confinement itself will break the time-reversal symmetry ( $\mathcal{T}$-breaking) in the absence of any external magnetic field. However, the $\mathcal{T}$-breaking due to mass potential confinement is different from that due to a magnetic field in a classical picture in which the field affects the particle orbit through the Lorentz force so that the geodesic motions are still characterized by straight lines within the confined domain. In the relativistic quantum theory of electrons, magnetic field is introduced through the form of minimal coupling in the corresponding vector potential that is different from the mass term. In the conventional $(3+1)$-dimensional spacetime, the mass itself cannot break the time-reversal symmetry. However, in $(2+1)$-dimensional systems (as for two-dimensional Dirac materials such as graphene and topological insulators), the mass term will induce chiral anomaly and hence $\mathcal{T}$ breaking. Thus, in the quantum realm, the $\mathcal{T}$-breaking caused by mass confinement also has a different origin from that due to the magnetic field [495, 496].

To better understand the physical origin of the mass confinement term $V \sigma_{z}$, one can consider a single particle in the absence of any magnetic field and compare the following two situations: (a) the particle is in a Dirac ring with hard-wall confinement of mass potential and (b) the particle is in a Schrödinger ring with the conventional, electrical potential (hard-wall) confinement. Assume that the classical orbits are identical for both cases. Apparently, in case (a), the $\mathcal{T}$-breaking is intrinsic but there is no $\mathcal{T}$-breaking in case (b). In fact, as indicated by Sir Michael Berry [93], the semiclassical origin of $\mathcal{T}$-breaking induced by mass confinement is quite intriguing. There can thus be unique phenomena caused by the mass confinement $V \sigma_{z}$ in $(2+1)$-dimensional Dirac systems. In fact, this type of confinement can be experimentally realized by exploiting the surface states of a three-dimensional topological insulator, where the mass term in the Dirac equation is originated from a Zeeman term induced by local exchange coupling.

Some basic properties of Eq. (228) are the following. Firstly, the confinement condition of imposing an infinite mass outside $\mathcal{B}$ naturally takes into account the Klein paradox for relativistic quantum particles and thus guarantees that the study is conducted in the single-particle framework, which is relevant to the intrinsic physics of a single Dirac cone in graphene or a topological insulator. Secondly, both reduced spatial dimension with mass confinement and applied magnetic flux can break the time-reversal symmetry of $\hat{H}:[\hat{\mathcal{T}}, \hat{H}] \neq 0$ if $V \neq 0$ or $\alpha \neq 0$, where $\hat{\mathcal{T}}=i \sigma_{y} \hat{K}$ and $\hat{K}$ denotes the complex conjugate. Thirdly, for $V=0$ and $\boldsymbol{A}=0$ in Eq. (228) (i.e., free massless particle), there are plane wave solutions whose positive energy part has the following form:

$$
\psi_{\boldsymbol{k}}(\boldsymbol{r})=\frac{1}{\sqrt{2}}\left(\begin{array}{c}
\exp \left(-i \frac{\theta}{2}\right) \\
\exp \left(i \frac{\theta}{2}\right)
\end{array}\right) \exp (i \boldsymbol{k} \cdot \boldsymbol{r})
$$


where $\boldsymbol{k}$ is a wave vector that makes an angle $\theta$ with the $x$ axis. Fourthly, by using the Dirac equation $i \partial_{t} \psi=\hat{H} \psi$ and defining $\rho=\psi^{\dagger} \psi$ as the local probability density, one has the following continuity equation

$$
\partial_{t} \rho+\nabla \cdot\left[\psi^{\dagger} v_{F} \hat{\boldsymbol{\sigma}} \psi\right]=0
$$

It is thus natural to define $v_{F} \hat{\boldsymbol{\sigma}}$ as the local current operator, so the local current density associated with state $\psi(\boldsymbol{r})$ is given by $\boldsymbol{j}(\boldsymbol{r}) \equiv v_{F} \psi^{\dagger} \hat{\boldsymbol{\sigma}} \psi=2 v_{F}\left[\operatorname{Re}\left(\psi_{1}^{*} \psi_{2}\right), \operatorname{Im}\left(\psi_{1}^{*} \psi_{2}\right)\right]$.

To obtain solutions of Eq. (228) requires a proper treatment of the boundary condition. For the Dirac ring system, it is convenient to use the infinite mass (also called Berry-Mondragon [93] or chiral bag [241, 497, 498]) boundary condition Eq. (63), as shown in Fig. 69. Substituting Eq. (63) into the current density formula, one can show that the boundary current

$$
\boldsymbol{j}(s)=2 v_{F}\left|\psi_{1}\right|^{2}(-\sin \theta, \cos \theta)
$$

is polarized along the boundary: clockwise and counterclockwise for the inner and outer edges, respectively. It is remarkable that this polarized property is independent of the shape of the confinement potential $V(\boldsymbol{r})$ and is thus protected from irregular boundary scatterings, even though the magnitude of the edge current can be affected.

An analysis of the general properties of the $\alpha$ (magnetic flux) dependent relativistic quantum spectrum $\left\{E_{j}(\alpha)\right\}$, as determined by Eq. (228) under the boundary condition Eq. (63), reveals that the first "Brillouin zone" is given by [137] $-1 / 2 \leq \alpha \leq 1 / 2$. The conformal-mapping method $[5,123,151]$ discussed in Sec. II can be used to calculate a large number of relativistic eigenvalues and eigenstates with high accuracy.

\section{Whispering gallery modes and superpersistent currents in a chaotic domain}

Irregular or chaotic boundary scattering can be generated by deforming the domain boundary so that the classical dynamics in the ring are chaotic. For example, the circular ring domain $\xi=0.5 \leq r \leq 1$ can be deformed by using the mapping

$$
w(z)=h\left[z+0.05 b z^{2}+0.18 b \exp (i \omega) z^{5}\right],
$$

where $\omega=\pi / 2, b \in[0,1]$ is the deformation parameter that controls the classical dynamics. As the value of the parameter $b$ is increased from zero to unity, the deformed ring will undergo a transition from being regular to mixed and finally to being fully chaotic. The normalization coefficient

$$
h=1 / \sqrt{1+\frac{b^{2}}{200}\left(1+\xi^{2}\right)+\frac{81 b^{2}}{500}\left(1+\xi^{2}+\xi^{4}+\xi^{6}+\xi^{8}\right)}
$$

guarantees that the domain area is invariant for arbitrary values of the deformation parameters $\{b, \omega, \xi\}$. Four representative domains are shown in the top row of Fig. 70 where, classically, the left most domain is integrable, the right most domain is fully chaotic, and the two middle domains have a mixed phase space. The middle and bottom rows of Fig. 70 show the lowest 10 energy levels as a function of the quantum flux parameter $\alpha$ (energy-flux dispersion), for Schrödinger and Dirac particles, respectively. It can be seen that $E_{j}(\alpha)=E_{j}(-\alpha)$ holds for the Schrödinger particle, but for the Dirac fermion, the symmetry is broken: $E_{j}(\alpha) \neq E(-\alpha)$. However, for both nonrelativistic and relativistic spectra, one has $E_{j}(\alpha)=E_{j}(\alpha+1)$. For the circular-symmetric ring 

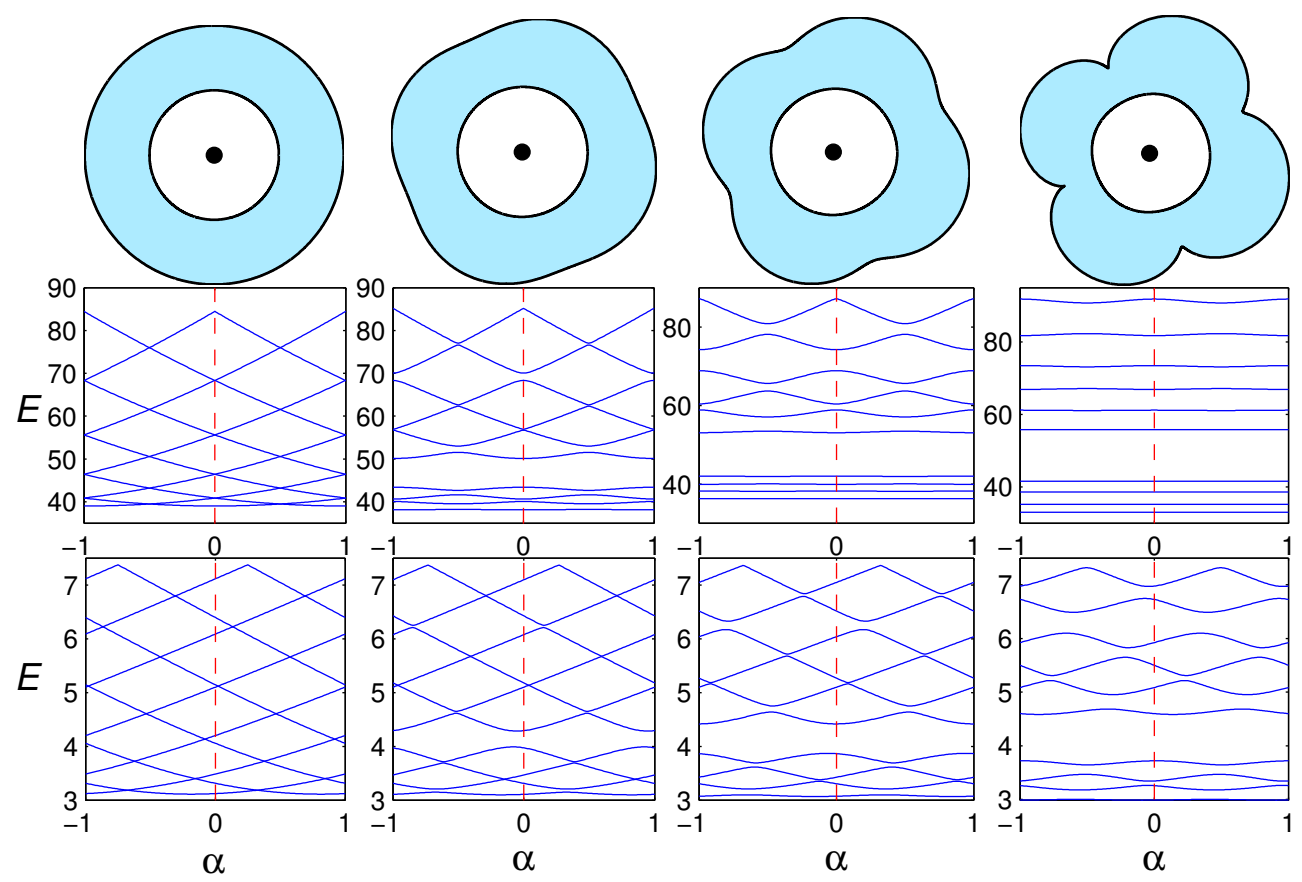

FIG. 70. A number of representative domain shapes with distinct classical dynamics and energy gap opening. Top panels: domain shape with classical dynamics ranging from integrable $(b=0$; left most panel) and mixed ( $b=0.25$ and 0.5 ; middle two panels) to chaotic ( $b=1.0$; right most panel). Middle panels: nonrelativistic $\mathrm{AB}$ oscillations (energy-flux dispersions). Bottom panels: relativistic $\mathrm{AB}$ oscillations. From Ref. [137] with permission.

$(b=0)$, AB oscillations in the energy levels have the period $\Phi_{0}$ (i.e., $\left.\alpha=1\right)$ and there are level crossings. Making the domain less symmetric by tuning up the value of the deformation parameter $b$ leads to classical mixed phase space (regular and chaotic), and eventually to full chaos $(b=1)$. It can be seen that, for the Schrödinger particle, the emergence of a chaotic component in the classical space leads to opening of energy gaps, generating level repulsion and flattening the $\mathrm{AB}$ oscillations associated with the corresponding eigenstates. However, for the Dirac fermion, the $\mathrm{AB}$ oscillations are much more robust against asymmetric deformations. Especially, for the fully chaotic case, $\mathrm{AB}$ oscillations for the Schrödinger particle disappear almost entirely while those for the Dirac fermion persist with amplitudes of the same order of magnitudes as for the integrable case.

Robust Dirac WGMs can arise in the fully chaotic AB ring domain. For low energy levels, the Schrödinger particle is strongly localized in the domain, as shown in Figs. 71(a-c), leading to a flat energy-flux dispersion. However, the Dirac fermion typically travels around the ring's boundaries, forming relativistic WGMs that persist under irregular boundary scattering due to chaos and are magnetic-flux dependent, as shown in Fig. 71(d-f). Conventional wisdom for Schrödinger particle stipulates that asymmetry in the domain geometry can mix/couple well-defined angular momentum states, opening energy gaps and leading to localization of states, so AB oscillations would vanish. However, for Dirac fermion, this picture breaks down - there are robust AB oscillations even in the fully chaotic domain and the particle tends to execute motions following the WGMs.

The robust $\mathrm{AB}$ oscillations in the chaotic Dirac ring lead to SPCs. The total persistent current 
(a)

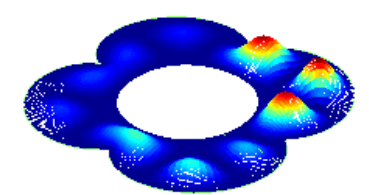

(d)

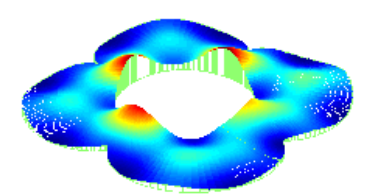

(b)

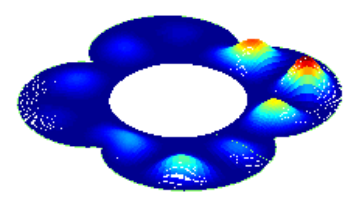

(e)

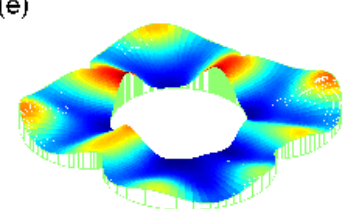

(c)

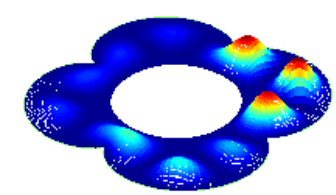

(f)

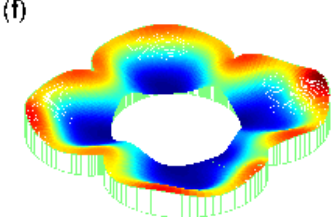

FIG. 71. Localized states in Schrödinger and whispering gallery modes in chaotic Dirac ring domains. Shown is the probability distribution of the 10th eigenstate for (a-c) nonrelativistic and (d-f) relativistic AB chaotic billiard for $\alpha=-1 / 4,0,1 / 4$, respectively. From Ref. [137] with permission.

(a)
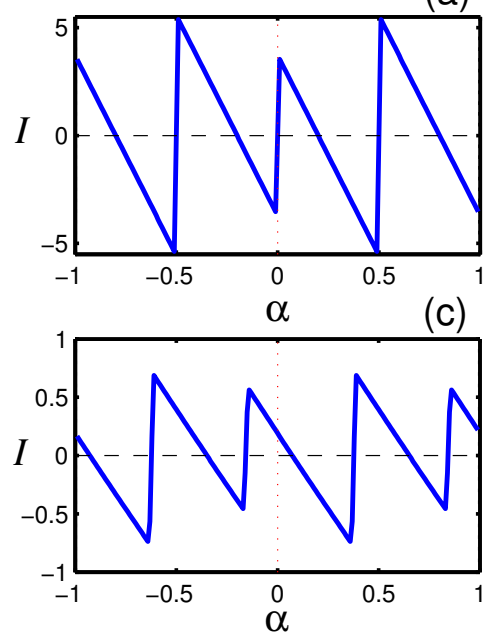

(b)
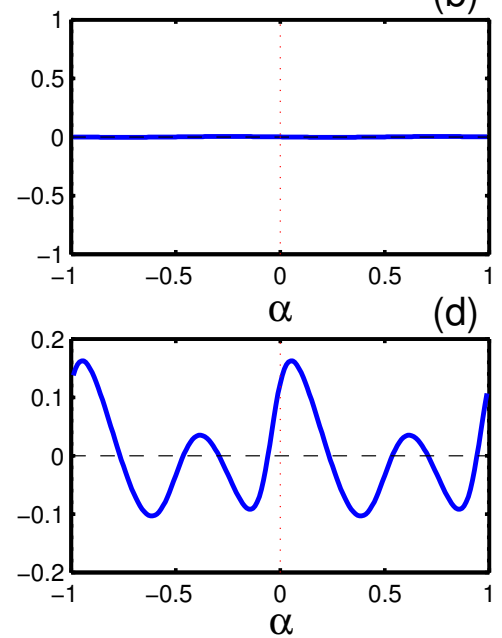

FIG. 72. Behaviors of the persistent currents in Schrödinger and Dirac ring domains. Shown are persistent currents as a function of the quantum flux parameter $\alpha$ associated with the five lowest states (including spin) for nonrelativistic $(a, b)$ and relativistic $(c, d)$ rings. The domain is integrable for $(a, c)$ and chaotic for (b,d). From Ref. [137] with permission.

can be calculated at zero temperature through $[182,251] I(\alpha)=-\sum_{j}\left(\partial E_{j} / \partial \alpha\right)$, where the sum runs over all occupied states with $E_{j}>0$. Due to the periodicity in the energy: $E_{j}(\alpha)=E_{j}(\alpha+1)$, the current is also periodic in $\alpha$ with the fundamental period $\alpha=1$. Figure 72 shows, for the nonrelativistic (top panels) and relativistic (bottom panels) rings, PCs resulted from the lowest five states (including spin) in regular (left column) and chaotic (right column) rings. It can be seen that, for classical integrable dynamics, PC oscillations display a common sawtooth form. However, at zero flux, PC is zero for the nonrelativistic ring [Fig. 72 (a)], while it has a finite value for the relativistic counterpart due to the breaking of the time-reversal symmetry. In the chaotic ring, the oscillations become smooth due to level repulsion in the corresponding energy-flux dispersion 
pattern. As a result, PCs carried by the Schrödinger particle practically vanish as compared with the integrable ring but the Dirac fermion still carries a persistent current with amplitude of the same order of magnitude as that for the integrable system - SPCs. Intuitively, SPCs carried by the Dirac fermion as an "exceptional" magnetic response are associated with the chaotic Dirac WGMs exemplified in Fig. 71.

\section{Origin of whispering gallery modes and superpersistent currents}

The origin of the "exceptional" magnetic response of the chaotic Dirac fermion can be understood through the behavior of the current carried by the particle at the boundary interface. An analytic understanding was obtained to predict the occurrence of Dirac WGMs and, consequently, SPCs [137], where the following setting was used: a plane wave incident obliquely on a straight potential jump $V(x, y)$ given by

$$
V(x, y)=\left\{\begin{array}{cc}
0, & x<0 \\
V_{0}, & x>0
\end{array}\right.
$$

as shown in Fig. 73. The incident wave $\psi^{i}$ is described by the wave vector

$$
\boldsymbol{k}_{0}=\left(k \cos \theta_{0}, k \sin \theta_{0}\right),
$$

the reflected wave $\psi^{r}$ by

$$
\boldsymbol{k}_{1}=\left(k \cos \theta_{1}, k \sin \theta_{1}\right)
$$

and the transmitted wave $\psi^{t}$ by

$$
\boldsymbol{u}=(i q, K),
$$

where $\theta_{1}=\pi-\theta_{0}$ and $K \equiv k \sin \theta_{0}, q$ has different forms for Schrödinger and Dirac systems. The situation of interest is where the energy of the incident wave satisfies $E<V_{0}$, which corresponds to total reflection.

The wave in region $\mathrm{I}(x<0)$ can be written as

$$
\Psi^{\mathrm{I}}(\boldsymbol{r})=\psi^{i}(x, y)+R \psi^{r}(x, y),
$$

and that in region II is

$$
\Psi^{\mathrm{II}}(\boldsymbol{r})=T \psi^{t}(x, y),
$$

where the coefficients $R$ and $T$ are to be determined by matching the waves at $x=0$. For comparison, it is necessary to treat the Schrödinger scalar wave and Dirac spinor wave separately.

Schrödinger scalar plane wave. For the nonrelativistic quantum system as shown in Fig. 73(b), one has, in region I $(x<0)$,

$$
\Psi_{S}^{\mathrm{I}}=\left(e^{i k x \cos \theta_{0}}+R e^{-i k x \cos \theta_{0}}\right) e^{i k y \sin \theta_{0}},
$$

and in region II $(x>0)$,

$$
\Psi_{S}^{\mathrm{II}}=T e^{-q x} e^{i K y},
$$

where $q$ and $K$ are related to each other through

$$
q=\sqrt{\frac{2 m\left(V_{0}-E\right)}{\hbar^{2}}+K^{2}} ; \quad E=\frac{\hbar^{2} k^{2}}{2 m},
$$


(a)

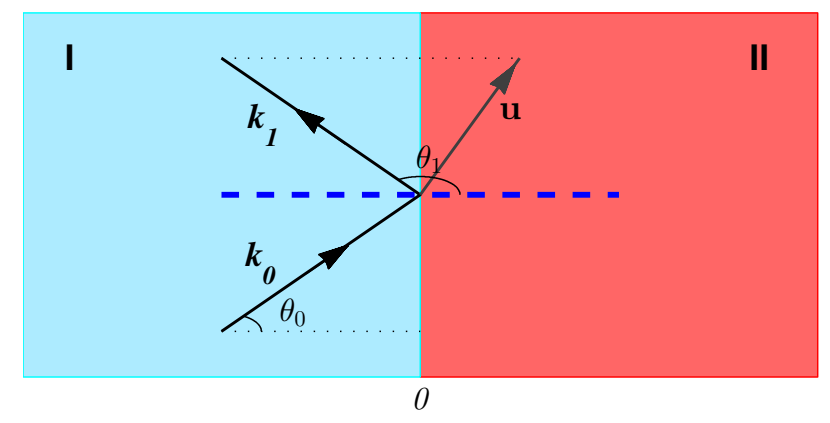

(b)

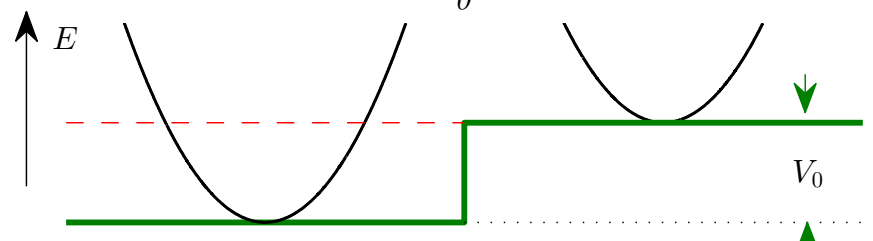

(c)

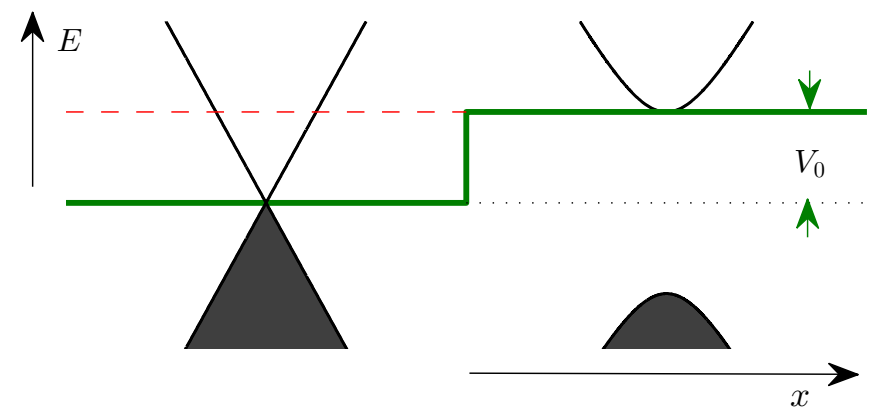

FIG. 73. Setting of analytic model to understand SPCs. Shown are incident, reflected and transmitted local plane waves at a potential jump. From Ref. [137] with permission.

with $m$ denoting the mass of the particle. Matching the waves and their derivatives at $x=0$, one gets

$$
\begin{aligned}
& R=\frac{k \cos \theta_{0}-i q}{k \cos \theta_{0}+i q}=e^{-2 i \beta}, \\
& T=\frac{2 k \cos \theta_{0}}{k \cos \theta_{0}+i q}=2 \cos \beta e^{-i \beta},
\end{aligned}
$$

where the parameter $\beta$ is defined via

$$
\tan \beta=\frac{q}{k \cos \theta_{0}} ; 0 \leq \beta \leq \frac{\pi}{2} .
$$

Given the wave function $\Psi_{S}(\boldsymbol{r})$, the associated probability current density is

$$
\boldsymbol{J}_{S}(\boldsymbol{r})=\frac{\hbar}{2 m i}\left[\Psi_{S}^{\dagger}(\boldsymbol{r}) \nabla \Psi_{S}(\boldsymbol{r})-\text { c.c. }\right],
$$

where c.c. denotes the complex conjugate. One obtains, in region I,

$$
\boldsymbol{J}_{S}^{\mathrm{I}}\left\{\begin{array}{l}
\left(J_{S}^{\mathrm{I}}\right)_{x}=\frac{\hbar k \cos \theta_{0}}{m}\left[1-|R|^{2}\right]=0 \\
\left(J_{S}^{\mathrm{I}}\right)_{y}=\frac{\hbar k}{m} \sin \theta_{0}\left[2+2 \cos \left(2 k x \cos \theta_{0}+2 \beta\right)\right]
\end{array}\right.
$$


and in region $\mathrm{II}$,

$$
\boldsymbol{J}_{S}^{\mathrm{II}}\left\{\begin{array}{l}
\left(J_{S}^{\mathrm{II}}\right)_{x}=0 \\
\left(J_{S}^{\mathrm{II}}\right)_{y}=\frac{\hbar K}{m}|T|^{2} e^{-2 q x}=\frac{\hbar k \sin \theta_{0}}{m} 4 \cos ^{2} \beta e^{-2 q x} .
\end{array}\right.
$$

In region I, the $y$ component of the probability current is the sum of two terms: (1) the term resulting from the sum of the currents associated with the incident and reflected waves, and (2) the term containing the factor $\cos \left(2 k x \cos \theta_{0}+2 \beta\right)$ that accounts for the interference between the incident and reflected waves. In region II, the probability current is also parallel to the $y$-axis, and it decays exponentially in the $x$-direction as an evanescent wave. Note that $\left(J_{S}^{\mathrm{II}}\right)_{y} \rightarrow 0$ as $q \rightarrow \infty$ (i.e., $V_{0} \rightarrow \infty$ ).

Planar Dirac spinor wave. The relativistic system shown in Fig. 73(c) can be treated in a similar manner. Expressing the wave in terms of massless spinor planar waves that are solutions of the Dirac equation, one has, in region I $(x<0)$,

$$
\Psi_{D}^{\mathrm{I}}=\frac{1}{\sqrt{2}}\left\{\left(\begin{array}{c}
e^{-\frac{1}{2} i \theta_{0}} \\
e^{\frac{1}{2} i \theta_{0}}
\end{array}\right) e^{i k x \cos \theta_{0}}+R\left(\begin{array}{c}
e^{-\frac{1}{2} i \theta_{1}} \\
e^{\frac{1}{2} i \theta_{1}}
\end{array}\right) e^{-i k x \cos \theta_{0}}\right\} e^{i k y \sin \theta_{0}},
$$

and in region II $(x>0)$,

$$
\Psi_{D}^{\mathrm{II}}=\frac{T}{\sqrt{2}}\left(\begin{array}{c}
-i \chi_{1} \\
\chi_{2}
\end{array}\right) e^{-q x} e^{i K y}
$$

where

$$
\chi_{1}=\sqrt{\frac{\left(V_{0}+E\right)(q-K)}{V_{0} q-E K}} ; \quad \chi_{2}=\sqrt{\frac{\left(V_{0}-E\right)(q+K)}{V_{0} q-E K}},
$$

and

$$
q=\sqrt{\frac{V_{0}^{2}-E^{2}}{\hbar^{2} v_{F}^{2}}+K^{2}} ; E=v_{F} \hbar k,
$$

with $v_{F}$ being the Fermi velocity.

Matching boundary conditions at $x=0$, one gets

$$
R=\frac{i \lambda-e^{i \theta_{0}}}{i-\lambda e^{i \theta_{0}}}=e^{i\left(2 \gamma+\theta_{0}-\frac{\pi}{2}\right)}
$$

where the parameters $\gamma$ and $\lambda$ are defined through

$$
\begin{gathered}
\tan \gamma=\frac{\chi_{1}-\chi_{2} \sin \theta_{0}}{\chi_{2} \cos \theta_{0}}=\frac{1-\lambda \sin \theta_{0}}{\lambda \cos \theta_{0}} \\
\lambda=\frac{\chi_{2}}{\chi_{1}}=\sqrt{\frac{\left(V_{0}-E\right)(q+K)}{\left(V_{0}+E\right)(q-K)}}=\frac{V_{0}-E}{\hbar v_{F}(q-K)}=\frac{\hbar v_{F}(q+K)}{V_{0}+E},
\end{gathered}
$$

and

$$
T=\frac{1}{\chi_{2}}\left[e^{\frac{1}{2} i \theta_{0}}+R i e^{-\frac{1}{2} i \theta_{0}}\right]=\frac{2 \cos \gamma}{\chi_{2}} e^{i\left(\gamma+\frac{\theta_{0}}{2}\right)} .
$$


For the spinor wave $\Psi_{D}(\boldsymbol{r})$ describing a Dirac fermion in two dimensions, the corresponding probability current density is

$$
\boldsymbol{J}_{D}(\boldsymbol{r}) \equiv v_{F} \Psi_{D}^{\dagger}(\boldsymbol{r}) \hat{\boldsymbol{\sigma}} \Psi_{D}(\boldsymbol{r})=2 v_{F}\left(\operatorname{Re}\left[\psi_{1}^{*} \psi_{2}\right], \operatorname{Im}\left[\psi_{1}^{*} \psi_{2}\right]\right)
$$

where $\hat{\boldsymbol{\sigma}}=\left(\sigma_{x}, \sigma_{y}\right)$ and $\Psi_{D}=\left[\psi_{1}, \psi_{2}\right]^{\mathrm{T}}$. One has, in region I,

$$
\boldsymbol{J}_{D}^{\mathrm{I}}\left\{\begin{array}{l}
\left(J_{D}^{\mathrm{I}}\right)_{x}=v_{F}\left(1-|R|^{2}\right) \cos \theta_{0}=0 \\
\left(J_{D}^{\mathrm{I}}\right)_{y}=v_{F}\left[2 \sin \theta_{0}+2 \sin \left(-2 k x \cos \theta_{0}+2 \gamma+\theta_{0}\right)\right]
\end{array}\right.
$$

and in region II,

$$
\boldsymbol{J}_{D}^{\mathrm{II}}\left\{\begin{array}{l}
\left(J_{D}^{\mathrm{II}}\right)_{x}=0 ; \\
\left(J_{D}^{\mathrm{II}}\right)_{y}=v_{F}|T|^{2} \chi_{1} \chi_{2} e^{-2 q x}=v_{F} \frac{4 \cos ^{2} \gamma}{\lambda} e^{-2 q x} .
\end{array}\right.
$$

Equations (231)-(232) and Eqs. (233)-(234) indicate that there are identical behaviors in the normal current densities (i.e., the $x$-component) for the nonrelativistic and relativistic rings, but there is a significant difference in the transverse current density (i.e., the $y$-component). In particular, for $E / V_{0} \rightarrow 0$ (the hard-wall limit), one has $\left.\left.\left(J_{S}^{\mathrm{I}}\right)_{y}\right|_{x=0} \equiv\left(J_{S}^{\mathrm{II}}\right)_{y}\right|_{x=0} \rightarrow 0$ for the nonrelativistic case, while $\left.\left.\left(J_{D}^{\mathrm{I}}\right)_{y}\right|_{x=0} \equiv\left(J_{D}^{\mathrm{II}}\right)_{y}\right|_{x=0} \rightarrow 2\left(1+\sin \theta_{0}\right)$ for the relativistic case. In addition, it can be seen from Eqs. (231)-(232) that $\left(J_{S}\right)_{y}$ is antisymmetric (odd function) with respect to $\theta_{0}$ so that its average over all possible incident angles, $\bar{J}_{S}^{y} \equiv \int_{-\pi / 2}^{\pi / 2} \mathrm{~d} \theta_{0}\left(J_{S}\right)_{y}$, is zero. The averaged transverse current density $\bar{J}_{D}^{y}$ in the relativistic counterpart, however, tends to a finite value in the hard-wall limit. A schematic comparison of the $y$ component of the probability current density $\boldsymbol{J}$ at the interface $(x=0)$ as a function of the incident angle $\theta_{0}$ and the magnitude of the reduced incident energy $E / V_{0}$ between the Schrödinger and Dirac systems is shown in Fig. 74. It can be seen that the nonrelativistic transverse current density $\left(J_{S}\right)_{y}$ is antisymmetric with respect to $\theta_{0}$, leading to zero contribution to $\bar{J}_{S}^{y}$, while the relativistic transverse current density $\left(J_{D}\right)_{y}$ is a nonnegative monotonic function of $\theta_{0}$, so there exists a finite transverse current even for a fully chaotic ring when all possible incident angles are taken into account. Such a finite averaged transverse current density $\bar{J}_{D}^{y}$ with magnitude one half the maximum makes it possible to form chaotic Dirac whispering gallery modes that carry considerable directional currents. This is the mechanism for the phenomenon of SPCs.

While Eqs. (233) and (234) appear different in form, at the interface $(x=0)$ they give exactly the same current density. For $V_{0} \rightarrow \infty$, the hard-wall boundary condition is restored (the infinitymass boundary condition). Physically the counterintuitive phenomenon can be understood, as follows. The incoming wave from the free region (mass term $V=0$ ) is spin polarized along its momentum (current) direction. After entering the potential region, a finite mass term acting on $\sigma_{z}$ will change the direction of the spin and hence affect the current via the spin-momentum locking term $\boldsymbol{k} \cdot \boldsymbol{\sigma}$. The relevant Hall-like phenomenon associated with the $\mathcal{T}$-breaking mass potential has been uncovered in a recent work [499]. In addition, the results do not depend on the special form of the wave function in region II (although such a symmetric form is convenient for analysis). In fact, the same results were obtained [106] when choosing the wavefunction to have the form $\sim[1, C(E)]^{T}$. 


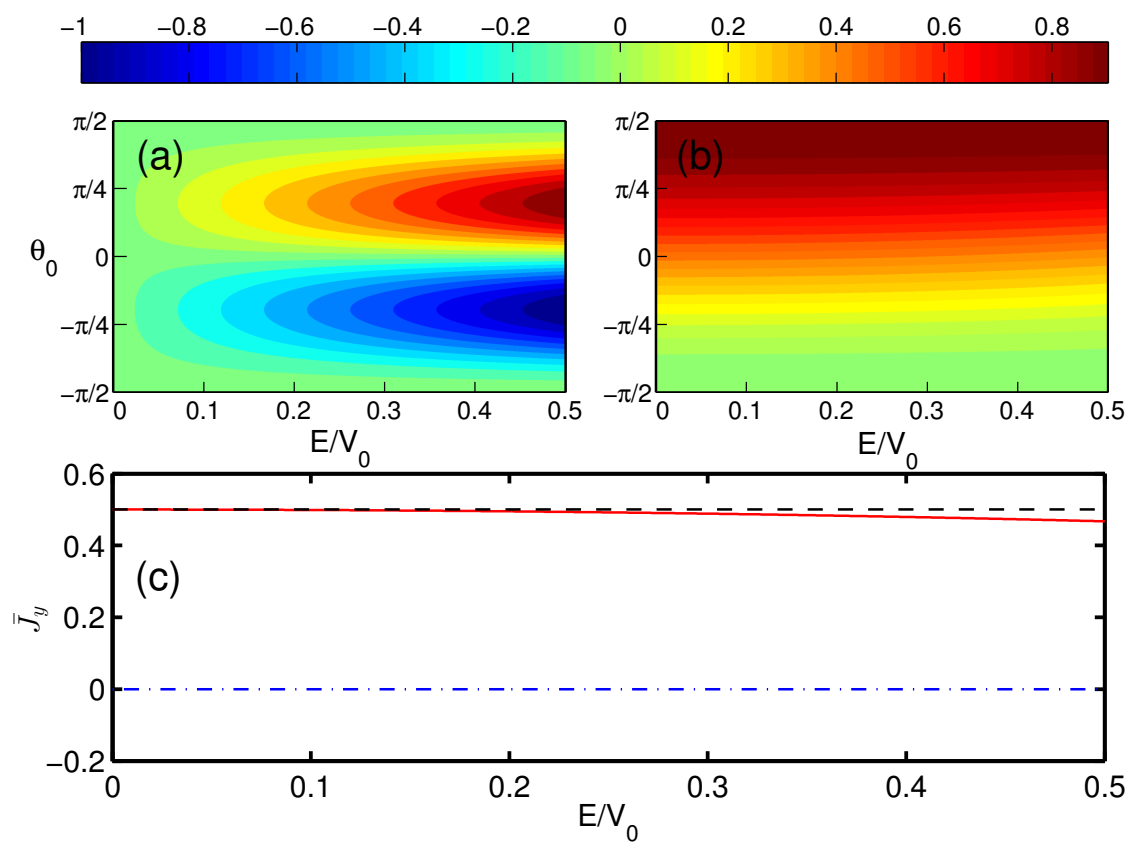

FIG. 74. Behaviors of the current density in nonrelativistic and relativistic quantum potential jump systems. Shown is a comparison of the $y$-component of the current density (coded by colors) as a function of the incident angle $\theta_{0}$ and the height of the reduced potential barrier $E / V_{0}$ between (a) nonrelativistic and (b) relativistic potential jump systems at the interface $(x=0)$. (c) The $y$-component of the current density averaged over all possible incident angles $\theta_{0}$ as a function of $E / V_{0}$ : blue (red) curve is for the nonrelativistic (relativistic) system and the black curve denotes the theoretical estimation of the relativistic system based on Eq. (234) with the assumption $E / V_{0} \ll 1$. In both cases, the values of the current density have been normalized by the respective maxima. From Ref. [137] with permission.

\section{Experimental scheme and robust relativistic qubit}

A possible experimental scheme to observe and characterize Dirac WGMs and SPCs is, as follows. A 3D topological insulator supports a (2D) gapless state on its surface, with low-energy excitations described by the massless Dirac Hamiltonian $[108,109] \hat{H}_{\text {surface }}=-i \hbar v_{F} \hat{\boldsymbol{\sigma}} \cdot \boldsymbol{\nabla}$, where $\hat{\sigma}$ represents the real spin. The surface electronic structure is similar to that of graphene, except that there is only a single Dirac point. Different from graphene, the Dirac surface states of a topological insulator are associated with strong spin-orbit interactions. In spintronics applications of topological insulators, it is desirable to introduce a gap into the surface states. This can be done by breaking the time-reversal symmetry using a ferromagnet insulator (FMI) deposited on the top of a topological insulator [341]. The exchange coupling induced due to proximity to the ferromagnet insulator will give rise to a local exchange field that lifts the Kramers degeneracy at the surface Dirac point and introduces a mass term into the Dirac Hamiltonian. Thus, in general, one has

$$
\hat{H}=v_{F} \hat{\boldsymbol{\sigma}} \cdot(-i \hbar \boldsymbol{\nabla}+e \boldsymbol{A})+V(\boldsymbol{r}) \hat{\sigma}_{z}+\gamma_{z} B \hat{\sigma}_{z},
$$

where the vector potential $\boldsymbol{A}$ accounts for the effect of the external magnetic field $\boldsymbol{B}=\boldsymbol{\nabla} \times$ $\boldsymbol{A}=B \hat{\boldsymbol{z}}$, with an additional Zeeman splitting correction in the last term. The controllable mass term $V \hat{\sigma}_{z}$ that is responsible for the local exchange coupling with an FMI cap layer makes 3D 


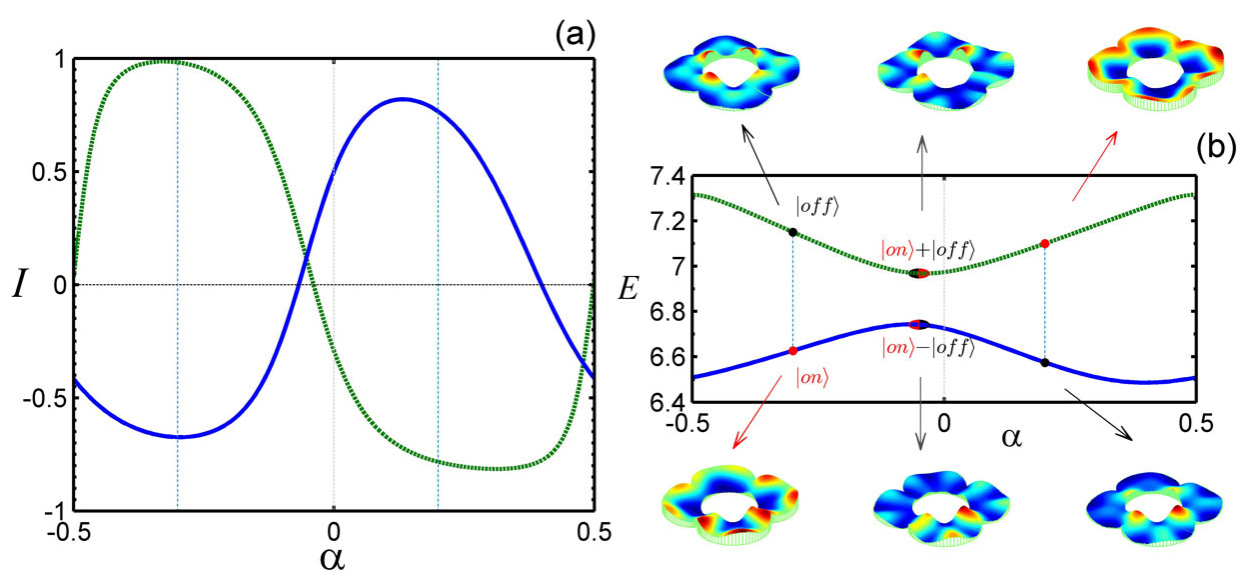

FIG. 75. Illustration of a flux-tunable qubit based on a pair chaotic Dirac WGMs. (a) "on-off" curves measured by the slope of the eigenenergies as a function of the flux (i.e., superpersistent current from a single level), (b) $\mid$ on $\rangle$ and $\mid$ off $\rangle$ states corresponding to the outer WGM carrying the maximum positive SPC and inner WGM carrying the minimum negative SPC, respectively. In (a,b), blue solid and green dashed lines correspond to the 9'th and 10'th energy-flux dispersions of the chaotic Dirac ring, respectively.

topological insulators a possible experimental platform for observing and characterizing Dirac WGMs and SPCs.

A potential application of Dirac WGMs lies in quantum information technology. Similar to the proposal of qubit in a two-dimensional topological insulator based on two different sets of helical edge states localized at the boundaries [482] and the idea of a charge qubit in a double quantum dot [500], a qubit system can be constructed [138] based on the chaotic Dirac WGMs guided by the inner and outer surfaces in opposite directions. Such an edge degree of freedom can be used to form a two-state system, denoted by the states $\mid$ on $\rangle$ and $\mid$ off $\rangle$ for the outer and inner chaotic Dirac WGMs, respectively. The set $\{\mid$ on $\rangle, \mid$ off $\rangle\}$ thus constitutes a complete diabatic basis. Generally, these two states correspond to two energy levels $\left\{E_{L}(\alpha), E_{U}(\alpha)\right\}$ with a rather larger difference (mismatch) and can be coupled and superpositioned for different values of the magnetic flux. As a result, two different levels of the system arise, say $\left\{E_{L}\left(\alpha^{\prime}\right), E_{U}\left(\alpha^{\prime}\right)\right\}$. The corresponding instantaneous eigenstates $\{|L\rangle,|U\rangle\}$ constitute an adiabatic basis. An effective $\alpha-$ dependent Hamiltonian describing the flux-tunable qubit can then be written in the adiabatic basis as

$$
\hat{H}_{\text {qubit }}^{a}(\alpha)=E_{L}(\alpha)|L\rangle\left\langle L\left|+E_{U}(\alpha)\right| U\right\rangle\langle U| .
$$

Expanding in the diabatic base [501], one has

$$
\left.\left.\hat{H}_{\text {qubit }}^{d}(\alpha)=-\frac{\tilde{\alpha}}{2}(\mid \text { on }\rangle\langle\text { on }|+| \text { off }\rangle\langle\text { off }|\right)-\frac{\Delta}{2}(\mid \text { on }\rangle\langle\text { off }|+| \text { off }\rangle\langle\text { on }|\right),
$$

and

$$
\left(\begin{array}{c}
|U\rangle \\
|L\rangle
\end{array}\right)=\left(\begin{array}{cc}
t_{-} & -t_{+} \\
t_{+} & t_{-}
\end{array}\right)\left(\begin{array}{l}
\mid \text { off }\rangle \\
\mid \text { on }\rangle
\end{array}\right)
$$

where $t_{ \pm}=\sqrt{(\Omega \pm \tilde{\alpha}) / 2 \Omega}, \Omega \equiv\left(E_{U}-E_{L}\right)=\sqrt{\Delta^{2}+\tilde{\alpha}^{2}}$, and $\tilde{\alpha}=\alpha+\alpha_{0}$, with $\Delta$ being the minigap (i.e., anticrossing energy) and $\alpha_{0}$ approximately characterizing the asymmetric degree 
of the system with respect to $\alpha=0$. Nonadiabatic transitions between the $\mid$ on $\rangle$ and $\mid$ off $\rangle$ states can be implemented by sweeping the magnetic flux parameter $\alpha$ at a finite rate. In particular, nonadiabatically tuning the flux such that $\tilde{\alpha}$ changes from $|\tilde{\alpha}| \gg \Delta$ to $\tilde{\alpha}=0$ (i.e., $\alpha=-\alpha_{0}$ ) drives the system from a pure $\mid$ on $\rangle$ (or $\mid$ off $\rangle$ ) to the minigap position where Rabi oscillations between $\mid$ on $\rangle$ and $\mid$ off $\rangle$ occur with the angular frequency given by $\Delta / 2: \cos (\Delta t / 2) \mid$ on $\rangle-i \sin (\Delta t / 2) \mid$ off $\rangle$. With respect to the stability of the qubit [482], a larger anticrossing energy $\Delta$ is desirable for enhancing the dephasing and relaxation times such that they are larger than the Rabi period $4 \pi / \Delta$.

A concrete example was given [138] to illustrate the idea of exploiting chaotic Dirac WGMs as a potential qubit scheme. Specifically, $E_{L}$ and $E_{U}$ were chosen to be the 9'th and 10'th energy levels, respectively, as shown in Fig. 75. In the chaotic AB billiard system, $\Delta$ is introduced by asymmetric distortions. Since the qubit is based on the chaotic Dirac WGMs that arise from a ring domain with severe boundary deformation, it will sustain sufficiently irregular scatterings and to be stable as well. In addition, since the chaotic Dirac WGMs are supported by the $\mathcal{T}$ breaking confinement (i.e., the mass-potential), the qubit is naturally robust against $\mathcal{T}$-breaking perturbations, making it superior, for example, to the qubit based on $\mathcal{T}$-protected edge states in two-dimensional topological insulator rings [482]. Practical issues such as the temperature effects were also addressed [138].

\section{Robustness of persistent currents in two-dimensional Dirac systems with disorders}

In real materials, impurities and defects are inevitable, raising the issue of robustness of the persistent currents. This issue was systematically addressed through an investigation of how the ensemble averaged current magnitude varies with the disorder density, where localized random potential was imposed to simulate the disorders [139]. Again, a Dirac ring domain with a vertical magnetic flux through the center was considered and the infinite mass boundary condition was used to constrain a Dirac fermion within the domain. Uncorrelated disorders throughout the domain were assumed, which can be simulated using localized, random electric potential uniformly distributed in the domain. In an experiment, for a given material, neither the strength nor the density of the disorders can be readily adjusted. However, the sample size can be controlled. Classically, under a vertical magnetic field, the electrons move along circular trajectories in the domain. In experiments, for a larger ring sample with constant disorder density, an electron encounters more disorders/scattering events in one complete rotation. It is thus convenient to fix the outer radius of the ring domain to be unity. In this case, varying the disorder density is equivalent to changing the size of the ring domain, where a higher density corresponds to a larger domain. Following this heuristic consideration, the disorder strength and the domain size were fixed but the density of disorders was systematically varied, and the total number of disorders in the whole domain can be used as a convenient control parameter. The Dirac equation was solved to obtain the magnitudes of the persistent currents as a function of the number of disorders. For comparison with the nonrelativistic quantum counterpart, the Schrödinger equation under the same setting was also solved. It was found [139] that, for the Dirac ring system, as the number of disorders is systematically increased, the average current decreases slowly initially and then plateaus at a finite nonzero value, indicating that the persistent currents are robust. WGMs were demonstrated to be the physical mechanism responsible for the robust currents. In contrast, in the nonrelativistic quantum ring system, the WGMs are sensitive and fragile to the disorders, leading to a rapid and exponential decay of the currents to zero. A physical theory was developed [139] based on 
a quasi one-dimensional approximation to understand the contrasting behaviors of the currents in the Dirac and Schrödinger rings.

In the presence of random disorders, the 2D Dirac Hamiltonian subject to a perpendicular magnetic field is

$$
\hat{H}=\hat{H}_{0}+U(\boldsymbol{r})=v_{F} \hat{\boldsymbol{\sigma}} \cdot(-i \hbar \boldsymbol{\nabla}+e \boldsymbol{A})+V(\boldsymbol{r}) \sigma_{z}+U(\boldsymbol{r}),
$$

where the disorders are modeled by a random electrical potential field $U(\boldsymbol{r}), V=0$ for $R_{1}<r<$ $R_{2}$, and $V=\infty$ for $r<R_{1}$ or $r>R_{2}$. The relevant lengths are normalized by the outer radius $R_{2}$ (e.g., $R_{2}=1$, the inner radius becomes $\xi=R_{1} / R_{2}$ ). In the absence of random disorders $U=0, \hat{H}=\hat{H}_{0}$, the Dirac equation in the ring domain can be solved analytically to yield a set of eigenenergies and eigenstates $\left\{E_{i}^{(0)},|i\rangle, i=1,2, \cdots\right\}$ (Sec. IIF 1). The radial part of the whole wavefunction can be expressed as a set of Hankel functions.

Treating the random disorders as perturbations, one has

$$
\sum_{i, j}\langle j|\hat{H}| i\rangle=\sum_{i} E_{i}^{(0)}+\sum_{i, j}\langle j|U(r, \theta)| i\rangle .
$$

The energy levels of the perturbed system can be solved numerically using the Hamiltonian in Eq. (238).

Numerical computations were carried out [139], as follows. The random disorders can be modeled through a set of uncorrelated Gaussian potential functions:

$$
U(r, \theta)=\sum_{s=1}^{N} U_{s}\left(r_{s}, \theta_{s}\right)=\sum_{s=1}^{N} u_{s} e^{-\delta r^{2} / 2 \sigma^{2}}
$$

where $s$ and $N$ are the index and the total number of random impurities in the domain, $\delta r$ is the distance between $(r, \theta)$ and $\left(r_{s}, \theta_{s}\right), \sigma$ and $u_{s}$ are the size and the potential height of a single electric impurity, respectively. The cutoff radius of any disorder was set to be $\delta r \leq 3 \sigma$ with $\left(R_{2}-R_{1}\right) / 20$ being the mean radius of the disorders. The strength of the disorders is randomly chosen from the interval $\left[-u_{m} / 2, u_{m} / 2\right]$, where $u_{m}$ is determined in terms of the average spacing of the first ten energy levels, $\overline{\Delta E}_{10}$.

For convenience, the superscripts " $D$ " and " $S$ " were used to denote the results for the Dirac and Schrödinger ring domains, respectively. Computations gave $\overline{\Delta E}_{10}^{(S)} \approx 10 \overline{\Delta E}_{10}^{(D)}$ for $\xi=1 / 2$. The maximum number of disorders is chosen to be 500 (corresponding to impurity area ratio $S_{\text {impurity }} / S_{\text {ring }} \simeq 0.43$ ) to prevent them from covering the ring domain completely. The single-level persistent current is defined as [502]

$$
I_{n}=\sqrt{\left\langle I_{n}^{2}(\Phi)\right\rangle}
$$

where $I_{n}(\Phi)=-\partial E_{n}(\Phi) / \partial \Phi$ is the flux-dependent persistent current associated with the $n$ 'th energy level and $\langle\cdot\rangle$ denotes disorder averaging. The experimentally measurable persistent current is given by $[455,502]$

$$
I^{t y p}(\Phi)=\sqrt{\left\langle\left[\sum_{n=1}^{n_{F}} I_{n}(\Phi)\right]^{2}\right\rangle}
$$



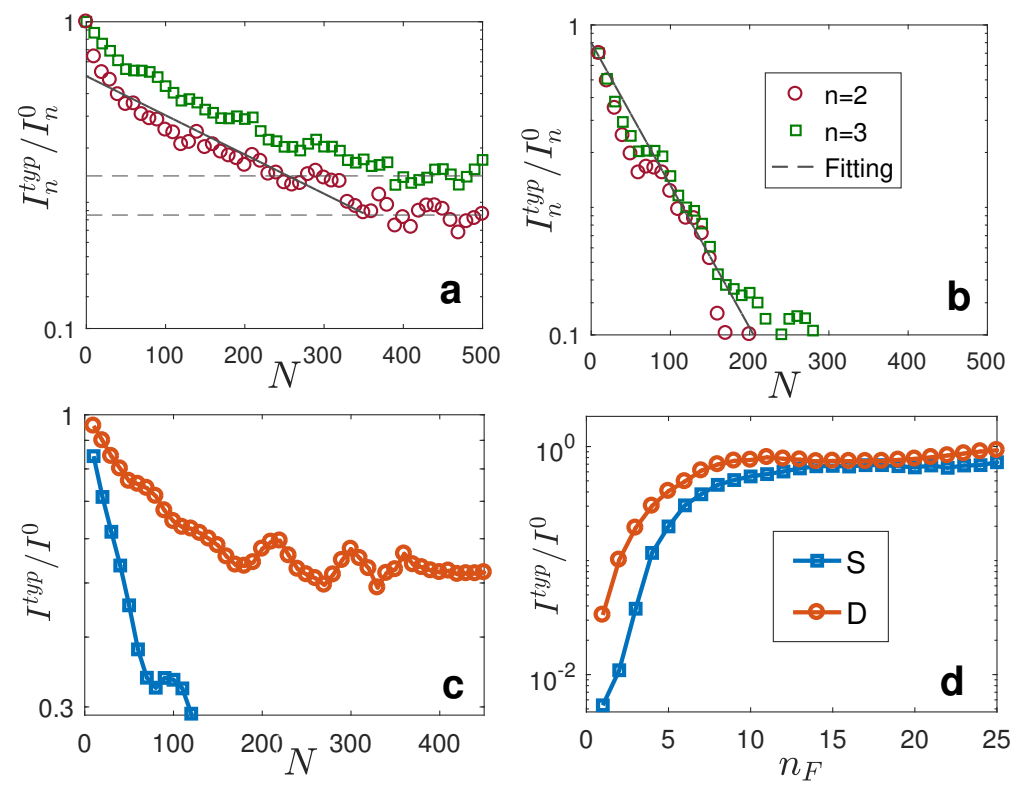

FIG. 76. Effects of random disorders on persistent currents in Dirac and Schrödinger rings subject to an external magnetic flux. Shown are typical single-level persistent currents versus the number of the disorders for (a) Dirac and (b) Schrödinger rings, for fixed disorder strength $u_{m}^{(D, S)}=300 \overline{\Delta E}_{10}^{(D, S)}$. The mean radius of a single impurity is $(1-\xi) / 20$. (c) Average persistent current versus the number of disorders for five levels below the Fermi energy, where other parameters are the same as in $(a, b)$ for the Dirac (denoted as " $D$ " and illustrated as circles) and Schrödinger (denoted as " $S$ " and displayed as squares) rings. The range of the number of disorders, $N \in[0,500]$, corresponds to the range of the ratio between the disorder and ring areas $S_{\text {impurity }} / S_{\text {ring }} \in[0,0.43]$. (d) Total persistent current versus $n_{F}$, the number of levels below the Fermi energy, for the Schrödinger and Dirac ring systems, where the number of impurities is 400. From Ref. [139] with permission.

where $n_{F}$ is the maximum energy level below the Fermi energy. The persistent currents averaged over the magnetic flux is written as $I^{t y p}=\overline{I^{t y p}(\Phi)}$. Generally, the persistent currents in Eqs. (240) and (241) are normalized by the corresponding disorder-free currents $I_{n}^{0}$ and $I^{0}$, respectively.

Figures 76(a) and 76(b) show, for the Dirac and Schrödinger systems, respectively, the typical single-level persistent currents versus the number $N$ of random disorders, which were calculated using $10^{2}$ statistical realizations. The error in the calculated value of the current amplitude is about $10^{-2}$. In both cases, the current amplitude decays exponentially for $0<N \lesssim 350$ (i.e., $\left.0<S_{\text {impurity }} / S_{\text {ring }} \lesssim 0.3\right): I_{n} \sim I_{n}^{0} \exp \left[-\gamma^{(D, S)} N\right]$, with the distinct feature that the decay rate for the Dirac system is about half of that for the Schrödinger system: $\gamma^{(D)} / \gamma^{(S)} \approx 1 / 2$. A more remarkable feature is that, for the Dirac system, after the initial exponential decay, the current amplitude approaches a constant value (which is about one order of magnitude larger than the numerical error) for $N \geq 350$, but for the corresponding Schrödinger system the current amplitude decays to a much smaller value. The decaying behaviors of the average persistent current associated with the five lowest energy levels for both the Dirac and Schrödinger rings are demonstrated in Fig. 76(c). It can be seen that, as more random disorders are introduced into the domain, the decaying behavior of the persistent currents is characteristically different for the Dirac and Schrödinger systems: for the former the currents are robust and continue to exist (in spite of dete- 
rioration in amplitude) but for the latter the currents quickly diminish. That is, persistent currents in the Dirac system are robust against random disorders.

The behavior of the total persistent current versus $n_{F}$, the number of levels below the Fermi energy, is shown in Fig. 76(d), where there are 400 random impurities in the ring domain for both the Schrödinger (blue squares) and Dirac (red circles) systems. The quantity $n_{F}$ is increased from 1 to the value at which the total persistent current is saturated. The currents for both systems increase with $n_{F}$ for $n_{F} \in[1,10]$ and become plateaued for further increase in the value of $n_{F}$. In the increasing phase, the persistent current of the Dirac ring system is much larger than that of the Schrödinger counterpart (note the logarithmic scale for the current). That is, for low energy levels (e.g., $n_{F} \leq 10$ ), disorders have a more devastating effect on the total persistent current for the Schrödinger system. However, the saturated current value for the Dirac ring is not significantly larger than that for the Schrödinger system (again note the logarithmic scale), due to the reason that the contributions to the total current from higher energy levels are less sensitive to disorders than those from the low energy levels.

A theoretical understanding of the results in Figs. 76(a-d) can be obtained using a quasi onedimensional model for both the Dirac and the Schrödinger rings, which can be derived from consideration of the dependence of the energy levels on the angular momentum quantum numbers [139].

An estimate of the maximally possible system size in which persistent currents can sustain can be obtained, as follows. In experimental studies, a 2D Dirac ring can be realized through the surface states of, e.g., $\mathrm{Bi}_{2} \mathrm{Te}_{3} / \mathrm{Bi}_{2} \mathrm{Se}_{3}$, with Fermi velocity about $v_{F} \approx 7 \times 10^{5} \mathrm{~m} / \mathrm{s}$ [503-505]. In the simulation, the Gaussian-like disorder is analogous to charge puddles of size $\sim 30 \mathrm{~nm}$ and strength $\sim 10 \mathrm{meV}$ associated with the surface states of, e.g., $\mathrm{Mn} / \mathrm{Ca}$-doped $\mathrm{Bi}_{2} \mathrm{Te}_{3} / \mathrm{Bi}_{2} \mathrm{Se}_{3}$ materials [505]. In a pure $\mathrm{Bi}_{2} \mathrm{Te}_{3} / \mathrm{Bi}_{2} \mathrm{Se}_{3}$ sample, the strength of the charge puddles is smaller than that for doped materials. One can thus set $u_{m} / 2=5 \mathrm{meV}$. In the computation, for the case of high disorder density, say $400-500$ impurities, the disorder pattern is similar to that of the charge puddles from experiments [505]. The maximum strength of the charge puddles is given by $u_{m}=300 \overline{\Delta E}_{10}$, with $\overline{\Delta E}_{10}=\hbar v_{F} \overline{\Delta k}_{10} / R_{2}$, where $k_{10} R_{2}=0.45$ and $R_{2}=300 \times 0.45 \hbar v_{F} u_{m} \approx$ $6 \mu \mathrm{m}$. As a result, the estimated size of the Dirac ring in which robust persistent currents can exist is $D=2 R_{2} \sim 12 \mu \mathrm{m}$, which is much larger than the maximum size of the normal metallic or semiconductor rings with persistent currents observed in previous experimental studies [184$189,506,507]$.

In a clean Dirac ring of size $D=12 \mu \mathrm{m}$, the persistent currents associated with one energy level can be estimated as $I_{n}^{0} \sim 2 \overline{\Delta E}_{10} / \Phi_{0}=0.45 \times \hbar v_{F} /\left(R_{2} \Phi_{0}\right) \approx 3 \mathrm{nA}$. Even if there are 500 impurities in the ring domain, there is still a finite persistent current: $I_{n} \approx 0.1 I_{n}^{0} \approx 0.3$ $\mathrm{nA}$. The total persistent current in an experimental system is given by $I=\sum_{n=1}^{N} I_{n}$, where the integer $N$ depends on the Fermi energy. For example, if the Fermi energy is $E=1 \mathrm{meV}$, several energy levels will be included. The total persistent current is $I \sim 1 \mathrm{nA}$, which can be observed in experiments, e.g., by using the SQUID technique [184-187, 189, 506]. 


\section{CONCLUSION AND DISCUSSION}

\section{A. Concluding remarks}

The traditional field of quantum chaos deals with the nonrelativistic quantum manifestations or fingerprints of classical chaos for systems described by the Schrödinger equation, for which the energy-momentum (dispersion) relation is parabolic. What are the relativistic quantum manifestations of classical chaos for systems described by the Dirac equation with a linear (hyperbolic) dispersion relation? This question, being fundamental to physics, has practical significance due to the tremendous development of Dirac materials in recent years. Here, we provide a comprehensive review of the main results obtained so far in the emergent field of relativistic quantum chaos in terms of physical properties/phenomena such as peculiar energy level-spacing statistics, scarring, chaotic scattering and transport, resonant tunneling, and persistent currents. We put a particular emphasis on behaviors that are uniquely relativistically quantum, i.e., those that do not occur in nonrelativistic quantum systems, which include chiral scars, significant residual resonance associated with transport through chaotic graphene quantum dots, unconventional statistics of energy level-spacing, manifestations of Klein tunneling, and an extraordinary degree of robustness of persistent currents in chaotic Dirac billiards. For example, while the study of energy level statistics is standard in traditional quantum chaos, it is surprising that GOE statistics can arise in graphene billiards even when the corresponding classical dynamics are integrable. Further, chiral scars in chaotic Dirac fermion systems are uniquely a relativistic quantum manifestation of certain classes of classical unstable periodic orbits with a specific orbital structure, which has no counterparts in nonrelativistic quantum systems. And, considering that in nonrelativistic quantum dot systems, chaos is capable of smoothing out sharp resonances, the persistence of such resonances associated with transport in chaotic graphene quantum dots is unexpected.

It is important to distinguish the field of relativistic quantum chaos from previous studies of nonlinear and chaotic behaviors in classical systems in special or general relativity [508-514]. A brief discussion of these works is as follows. In an earlier work [508], ideas from nonlinear dynamics were used to construct a new class of classical physical systems with nonlinearity arising from complicated gravitational effects. In Ref. [509], the classical three-body problem was studied, where the motion of a charged particle is governed by a static electric field from two fixed, equal charges in space under special relativity, and the intrinsic nonlinear, relativistic kinematics generates nonintegrable dynamics. In Ref. [510], chaotic geodesic scattering was studied in radiative plane wave spacetime defined by a particular metric form through an equivalent classical Hénon-Heiles Hamiltonian. Classical chaotic scattering was also investigated in general relativistic systems in the presence of a Yang-Mills field [511]. In another work [512], imaginary mass tachyon physics was studied in open hyperbolic space with constant negative curvature through a field theory approach that is similar to studying classical electrodynamics through the photon field. Due to the open, multi-connected, and hyperbolic cosmic space, classical chaos can arise, and the quantum effect can be treated by including the induced tachyon potential into the Schrödinger equation as a perturbation to the Coulomb potential in the local Euclidean limit. Recently, Ref. [513] studied the relativistic effects in the chaotic Sitnikov problem, and Ref. [514] investigated the global relativistic effects in chaotic scattering. In spite of the fact that these previous works dealt with nonlinear and chaotic dynamics in classical relativistic systems, they are not pertinent to the field of relativistic quantum chaos as reviewed in the present article, as the purposes 
of these works were not to study the relativistic quantum manifestations of classical chaos.

It is worth emphasizing that the field of relativistic quantum chaos is not just some standard machinery of quantum chaos applied to relativistic quantum systems. In terms of fundamental science, there are uniquely relativistic quantum phenomena such as Klein tunneling, Zitterbewegung, and pair creations that find no counterparts in nonrelativistic quantum systems. When classical chaos "meets" with them, strikingly new and counterintuitive phenomena can arise. At the present, the interplay between classical chaos and fundamentally (pseudo-) relativistic quantum entities and phenomena such as the intrinsic spin degree of freedom, valley degree of freedom, symmetry anomalies and Klein tunneling, is largely unknown, calling for attention from the physics community to the emergent field of relativistic quantum chaos.

The most compelling reason that motivated the study of relativistic quantum manifestations of classical chaos was the vast research on graphene that started around 2004 and the subsequent discoveries/development of a wide variety of 2D Dirac materials. Indeed, the often "unusual" manifestations of classical chaos in solid state systems described by relativistic quantum mechanics can be exploited for potential applications. To investigate the effects of chaos on the physics of electronic, spintronic, and even valleytronic systems has the potential to improve device performance. It is possible that novel concepts and cutting-edge devices would arise from research on the classical-relativistic quantum correspondence. There were excellent reviews on graphene [105107] and topological insulators [108, 109]. Although the present Review employs these novel 2D materials as the potential application background, it should be noted that these are not the focus. Instead, it provides a comprehensive account of the emergent field of relativistic quantum chaos that treats the fundamental interplay between classical chaos and relativistic quantum mechanics.

\section{B. Unsolved problems in relativistic quantum chaos at the present}

We conclude this Review by speculating about a number of open problems in relativistic quantum chaos.

\section{Relativistic quantum-classical correspondence}

We have demonstrated in this Review that when classical nonlinear dynamics and chaos meet with relativistic quantum mechanics as described by the Dirac equation, unusual and unexpected phenomena can arise. For example, the inherent chirality of the massless Dirac fermions and the subtle time-reversal symmetry breaking due to hard wall confinement make certain quantum scars chiral. A broken time-reversal symmetry can lead to parity anomaly, a phenomenon stipulated by the Dirac theory for fermions in $(2+1)$-dimensional spacetime. The fundamental parity symmetry associated with the classical action does not mean that the same symmetry would arise in the corresponding relativistic quantum system. At the present, not much has been known about the relativistic quantum-classical correspondence in the context of the Dirac quantum theory.

\section{Semiclassical theory for relativistic quantum scars}

Scars are remarkable quantum fingerprints of classical unstable periodic orbits, the most fundamental building blocks of any nonlinear dynamical system. In nonrelativistic quantum sys- 
tems, a comprehensive understanding of scarring was obtained through the semiclassical analysis $[1,53,64,78]$. In particular, the semiclassical expression of the density of states can be obtained by taking the trace of the Green's function:

$$
\rho(E)=\sum_{j} \delta\left(E-E_{j}\right) \approx-(1 / \pi) \int \operatorname{Im} G_{\mathrm{sc}}(\boldsymbol{q}, \boldsymbol{q}, E) \mathrm{d} \boldsymbol{q}
$$

where

$$
\begin{aligned}
G_{\mathrm{sc}}(\boldsymbol{q}, \boldsymbol{q}, E) & =G_{0}(\boldsymbol{q}, E)+G_{o s c}(\boldsymbol{q}, E), \\
\rho(E) & \approx \rho_{0}(E)+\rho_{\mathrm{osc}}(E),
\end{aligned}
$$

and $G_{0}(\boldsymbol{q}, E)$ is the contribution of the so-called "zero length trajectories" arising from the Thomas-Fermi approximation, $G_{\mathrm{osc}}(\boldsymbol{q}, E)$ is the contribution of classical trajectories with a nonzero action starting and ending at the same point $\boldsymbol{q}$, and $\rho_{0}$ and $\rho_{\text {osc }}$ are the density of states from $G_{0}$ and $G_{\text {osc }}$, respectively. The celebrated Gutzwiller trace formula [324, 515-518] gives the oscillatory part of the density of states in terms of a sum over isolated classical periodic orbits, based on the Van Vleck formula for the semiclassical propagator under the stationary phase approximation. The quantum scars can be explained as the nonuniform averaged wavefunction square represented as the sum over a finite number of periodic trajectories in the configuration space $[53,78]$. For a specific periodic orbit, $G_{\text {osc }}$ can be calculated and one can find certain scars corresponding to the orbit [53]. Berry gave an explanation in the phase space [1]. Agam and Fishman proceeded further to predict the scarring of specific wavefunctions of chaotic systems [64]. For chaotic Dirac fermion systems, the semiclassical propagator and the trace formula have been obtained in the spirit of the Gutzwiller trace formula through a careful treatment of the spin contributions to the semiclassical propagator [442]. The expression for the semiclassical Green's function was used in graphene with an electrostatic potential and/or a mass confinement where the presence of a semiclassical phase was found and the density of states was calculated [519, 520]. The novel features that arise in the case of the Dirac equation are the spin degree of freedom and its coupling to the momentum and the boundaries. In spite of the previous efforts, at the present, a comprehensive semiclassical theory for relativistic quantum scars is missing.

\section{Interplay between Klein tunneling and classical dynamics}

In relativistic quantum mechanics, a fundamental phenomenon that has no counterpart in nonrelativistic quantum systems is Klein tunneling, tunneling through a finite potential barrier with probability one, even when the particle energy is less than the barrier height and the barrier width is many times of the wavelength. As demonstrated in Sec. V, Klein tunneling can leave unmistakable fingerprints in quantum resonant tunneling in that, even when the particle energy is near zero, the tunneling rate can be large, and this holds regardless of whether the classical dynamics are integrable or chaotic. In a graphene quantum point contact, abnormal electron paths can arise due to Klein tunneling in that the electrons tend to move along paths in a potential field which are classically forbidden, leading to fractal-like conductance fluctuation patterns [126]. In relativistic quantum chaos, a natural question thus concerns about the interplay between classical chaos and Klein tunneling. For spin-1/2 particles, the occurrence of Klein tunneling requires certain specific incident angle of the particle onto a potential barrier. In this case, one may intuitively expect 
that chaos would facilitate the occurrence of Klein tunneling due to the random angle distribution associated with classically chaotic trajectories. A possible consequence is that it may be more difficult to confine spin-1/2 fermions in a finite domain when there is chaos than when the classical dynamics are integrable. For spin-1 particles arising in solid material or photonic systems, the phenomenon of super-Klein tunneling can occur where, when the particle energy is a certain fraction of the barrier height, the tunneling probability is one, regardless of the incident angle [521]. In this case, intuition stipulates that it may not be possible to confine such particles. At the present, the interplay between Klein tunneling and different types of classical dynamics is not understood.

\section{Effect of many body interactions on relativistic quantum chaos}

In condensed matter systems, many body interactions (e.g., electron-electron interactions) lead to fascinating phenomena such as superconductivity and fractional quantum Hall effect. In the field of quantum chaos, the standard setting was that of single particle dynamics, in which manybody effects were ignored. While there were previous studies of the interplay between many-body interactions and classical chaos [202-205, 446, 447], these were exclusively for nonrelativistic quantum systems. The interplay between classical dynamics and many-body interactions was studied [136] in the specific setting of relativistic quantum tunneling in graphene systems based on the Hubbard model [206]. At the present, to develop a general understanding of the effect of chaos on relativistic quantum systems with many-body interactions is an open problem.

\section{Interplay between spin and nonlinear dynamics}

Spin is a fundamental and intrinsic quantity in relativistic quantum systems. Although there is no classical correspondence for spin, classical dynamics could still have influence on spin transport. The interplay between classical chaos and spin should thus be an important branch of the field of RQC. A recent work [144] studied the effect of chaos on spin polarization in graphene quantum dots. Specifically, when graphene is placed on a substrate of heavy metal, Rashba spinorbit interaction $[522,523]$ of substantial strength can occur, leading to spin polarization. In the study [144], Rashba interaction is confined within the central scattering region whose geometrical shape can be chosen to yield distinct types of dynamics, e.g., regular or chaotic, in the classical limit. It was found that, for spin unpolarized incident electrons, in the experimentally feasible range of the Rashba interaction strength, the average polarization for a chaotic dot can be markedly larger than that for a regular or mixed dot. From the semiclassical viewpoint, a key quantity that determines the average spin polarization is the angle distribution of the outgoing electrons at the interface between regions with and without the Rashba interaction, respectively. Classical chaos provides a wider distribution that leads to higher average spin polarization. Exploiting the role of chaos in manipulating spin and/or associated helicity can have potential applications in Dirac material based spintronics. There has been little work in this area.

Recently, a hybrid type of relativistic quantum/classical nonlinear dynamical systems has emerged [524, 525]. Consider a 3D topological insulator subject to a weak electrical field, where a spin polarized surface current is generated [108, 109, 526]. When an insulating, ferromagnetic material is placed on top of the topological insulator, the surface current will cause the magnetization vector of the ferromagnet to evolve with time, as described by the classical Landau- 
Lifshitz-Gilbert (LLG) equation [527]. More specifically, the physical interactions between the topological insulator and ferromagnet are as follows. The robust spin polarized current on the surface of the topological insulator generates [528] a strong spin-transfer torque [529] to the ferromagnet, inducing a change in its magnetization vector. The ferromagnet, in turn, generates a proximity induced exchange field in the topological insulator. As a result, there is an exchange coupling term in the surface Dirac Hamiltonian of the topological insulator, which modulates the quantum transmission and leads to a change in the surface current. The whole coupled system is thus of a hybrid type: relativistic quantum topological insulator and classical ferromagnet. The interactions render time dependent the dynamical variables in both the topological insulator and ferromagnet: the surface current for the former and the magnetization vector for the latter. Due to the intrinsic and externally spin-transfer torque induced nonlinearity of the LLG equation, the whole configuration represents a novel type of hybrid nonlinear dynamical systems, in which a rich variety of phenomena such as bifurcations, chaos, synchronization, and multistability can arise [525]. To investigate such hybrid relativistic quantum/classical nonlinear systems can have practical values. For example, because of the dissipationless and spin-momentum locking nature of the surface currents of the topological insulator, little power is needed for generating a significant current, making the system appealing for potential applications in next generation of low power memory devices.

\section{Relativistic quantum chaos in pseudospin-1 systems}

Searching for novel types of excitations (or quasiparticles) beyond the prototypical Dirac fermions is of particular interest to the broad scientific community [530-532]. There are new materials whose energy bands consist of a pair of Dirac cones and a flat band, electronic or optical. For example, in a dielectric photonic crystal, Dirac cones can be induced through accidental degeneracy that occurs at the center of the Brillouin zone. This effectively makes the crystal a zero-refractive-index metamaterial at the Dirac point where the Dirac cones intersect with another flat band [533-537]. Alternatively, configuring an array of evanescently coupled optical waveguides into a Lieb lattice [538-541] can lead to a gapless spectrum consisting of a pair of common Dirac cones and a perfectly flat middle band at the corner of the Brillouin zone. As demonstrated recently, loading cold atoms into an optical Lieb lattice provides another experimental realization of the gapless three-band spectrum at a smaller scale with greater dynamical controllability of the system parameters [542]. Theoretically, it is necessary to use the the generalized Dirac-Weyl equation for massless spin-1 particles [543] to describe these materials. Recent works on scattering of pseudospin-1 particles from a simple circular potential disk [521, 544] revealed a number of unusual physical phenomena. For example, for small disk size, a surprising revival resonant scattering phenomenon can occur, with the physical mechanism being a peculiar type of boundary trapping profile through the formation of unusual vortices. In the semiclassical regime, a perfect caustic phenomenon emerges as a result of the super-Klein tunneling effect in which the transmission probability is one for any incident angle, leading to the scatterer's being effectively as a Veselago lens. In the far scattering field, an unexpected isotropic behavior emerges at low energies, which can be attributed to the vanishing Berry phase for massless pseudospin-1 particles and, consequently, to constructive interference between the time-reversed backscattering paths. For weak scatterers in the subwavelength regime where the scatterer size is much smaller than wavelength, the phenomenon of superscattering [544] that defies the conventional Born theory in optics can 
arise, which manifests itself as unusually strong scattering characterized by extraordinarily large values of the cross section even for arbitrarily weak scatterer strength. At the present, the interplay between classical chaos and the generalized Dirac-Weyl physics is completely unknown.

\section{Role of chaos in valley filtering}

In two-dimensional Dirac materials, in addition to charge and spin, valley quantum numbers provide an alternative way to designate and distinguish quantum states. For example, for graphene there are two nonequivalent Dirac points (denoted as $\boldsymbol{K}$ and $\boldsymbol{K}^{\prime}$ ) in the first Brillouin zone, and the distinct momenta associated with them represent the valley quantum numbers [482]. Because of the large momentum separation, the two nonequivalent valleys act as an ideal two-state system that is robust against external perturbations $[270,545]$. In addition to graphene, binary valley characteristics can also arise in other materials such as silicene and $\mathrm{MoS}_{2}$ [546], graphene-inspired artificial crystals such as photonic graphene [547] that exhibits valley-polarized beams and sonic (phononic) crystals [548] in which valley vortex states can emerge, as well as valley photonic crystals [549]. Generally, quasiparticles carrying distinct valley quantum numbers can be exploited for applications such as quantum information processing [550], leading to the concept of valleytronics [270, 359], a field that has attracted much recent interest [420, 546, 548, 551-556].

Valley filtering [359], the process to separate the electrons with distinct valley quantum numbers, is a fundamental issue in valleytronics. The existing approach to valley filtering is to exploit the valley Hall effect (VHE) [270, 553, 554, 557-564], which makes quasiparticles with different valley quantum numbers to separate from each other and move in spatially distinct regions. For the honeycomb lattice system (e.g., graphene), valley-resolved, gap-opening perturbations are necessary for VHE and valley filtering [270, 559]. Alternative mechanisms for VHE require external magnetic fields, strain-induced pseudo magnetic fields, or magnetic materials that have an opposite effect on the two valleys [420, 556, 565-567]. A recent work [568] demonstrated, however, that VHE can be realized without any valley-resolved perturbation.

Can classical chaos be exploited for modulating VHE and what is the role of chaos in valley filtering? These and related questions are completely open at the present.

\section{Interplay between classical chaos and topological quantum phases}

At a fundamental level, quantum anomalies are at the forefront of condensed matter physics and material science in pursuit of topological phases of matters (topological quantum matters) first studied by Semenoff [569] and Haldane [570] in lattices and narrow-gap semiconductors. The emergent anomalies are typically relativistic quantum phenomena and induce intriguing quantumclassical correspondence in terms of the underlying fundamental symmetries, which are then relevant to relativistic quantum chaos but not to nonrelativistic quantum chaos. When taking into account the intrinsic spin degree of freedom and the striking symmetry anomalies, the study of relativistic quantum chaos represents a new twist that does not exist in the study of traditional quantum chaos. Exciting new physics can be anticipated through the study of the manifestations of classical chaos in Dirac materials and topological quantum systems. 


\section{Role of chaos in time-dependent, relativistic quantum systems}

Classically, irregular constraints in the confinement geometry and/or spatial disorders can lead to random or chaotic dynamics. Another route to generating complex dynamics is through modulations in time, which occurs in driven physical systems/processes involving time dependent interactions. One representative class of systems is one-dimensional, nonrelativistic nonlinear oscillators, whose classical motion can be chaotic in the presence of a time dependent force. For example, when there are cubic and/or higher order terms in the potential, a sinusoidal driving force can induce chaos. A similar situation can occur in the relativistic counterpart with the exception that an anharmonic potential is no longer required for chaos because relativistic nonlinearity, i.e., nonlinearity arising from the relativistic kinetic energy term in the Hamiltonian, can play a similar role to that by the anharmonic terms in the corresponding nonrelativistic system. A driven relativistic harmonic oscillator can thus serve as a unique model system to study relativistic quantum nonlinear dynamics, where the role of relativity is particularly relevant. Another advantage is that, in the nonrelativistic limit, analytical solutions can be obtained. There was a previous study of the relativistic quantum and classical dynamics through the time dependent Dirac equation and the associated relativistic Liouville equation [571]. A surprising finding was that the relativistic quantum evolution agrees with the predictions based on the classical phase-space density remarkably well, regardless of the emergence of relativistic classical chaos. In fact, there are distinct evolution dynamics of the width of the wave packet in the relativistic and nonrelativistic regimes. Especially, for the relativistic quantum system, the width exhibits an oscillatory pattern before settling down to a nearly steady value. However, for the nonrelativistic system, the width does not depend on time, as revealed by closed solutions.

Another paradigm for studying driven quantum nonlinear dynamics from the perspective of quantum chaos or quantum-classical correspondence is the kicked rotor [572]. While most such studies were for nonrelativistic systems, there were exceptions that treated the relativistic extensions [573-575]. In particular, solutions of both the classical and quantum dynamics of the relativistic kicked rotor [573] enabled the underlying transport properties to be studied, which is characterized by the time dependent behavior of the system energy. It was found [573] that quantum resonances are absent in the relativistic regime. Moreover, there is a high degree of similarity between the classical and quantum dynamics, effectively making the relativistic kicked rotor an integrable system. An analysis of the transport dynamics in the momentum space in the long time regime revealed a remarkable super-ballistic behavior [574]. The underlying physics of this phenomenon can be attributed to the unique relativistic dispersion relation with a finite mass, which is approximately quadratic for low momentum values but linear in the high momentum regime. As a result, anomalous transport dynamics can arise in the momentum space. A fairly recent study [575] on the system of up to three coupled relativistic kicked rotors incorporating intricate interaction effect revealed that localized phase can persist.

At the present, the interplay between chaos and relativistic quantum mechanics in time dependent systems remains largely unexplored. 


\section{ACKNOWLEDGEMENT}

Generations of Ph.D. students at Arizona State University and later at Lanzhou University were involved in research on relativistic quantum chaos. They are: Dr. Ryan Yang, Dr. Xuan Ni, Dr. Guanglei Wang, Dr. Lei Ying, Dr. Rui Bao, Mr. Pei Yu, Mr. Ziyuan Li and Mr. Chengzhen Wang. We thank them. We would also like to thank Dr. Arje Nachman at the Air Force Office of Scientific Research and Dr. Michael Shlesinger from the Office of Naval Research for their great support over the years - without which the works on relativistic quantum chaos would not have been possible. YCL is currently supported by the Pentagon Vannevar Bush Faculty Fellowship program sponsored by the Basic Research Office of the Assistant Secretary of Defense for Research and Engineering and funded by the Office of Naval Research through Grant No. N0001416-1-2828. LH was supported by NNSF of China under Grants No. 11422541 and No. 11775101. 
[1] M. V. Berry, Quantum scars of classical closed orbits in phase-space. Proc. R. Soc. London Series A Math. Phys. Eng. Sci. 423, 219-231 (1989).

[2] H.-J. Stöckmann, Quantum Chaos: An Introduction (Cambridge University Press, New York, 2006).

[3] F. Haake, Quantum Signatures of Chaos, Springer series in synergetics (Springer-Verlag, Berlin, 2010), third edn.

[4] M. V. Berry, Semiclassical theory of spectral rigidity. Proc. R. Soc. London Series A Math. Phys. Eng. Sci. 400, 229-251 (1985).

[5] M. V. Berry, M. Robnik, Statistics of energy-levels without time-reversal symmetry - AharonovBohm chaotic billiards. J. Phys. A Math. and Gen. 19, 649-668 (1986).

[6] M. Robnik, M. V. Berry, False time-reversal violation and energy level statistics: The role of antiunitary symmetry. J. Phys. A Math. Gen. 19, 669 (1986).

[7] B. V. Chirikov, A universal instability of many-dimensional oscillator systems. Phys. Rep. 52, 263 379 (1979).

[8] B. V. Chirikov, A theory of quantum diffusion localization. Chaos 1, 95-100 (1991).

[9] O. Bohigas, M. J. Giannoni, C. Schmit, Characterization of chaotic quantum spectra and universality of level fluctuation laws. Phys. Rev. Lett. 52, 1-4 (1984).

[10] G. Casati, B. V. Chirikov, I. Guarneri, Energy-level statistics of integrable quantum systems. Phys. Rev. Lett. 54, 1350-1353 (1985).

[11] B. V. Chirikov, D. L. Shepelyansky, Shnirelman peak in level spacing statistics. Phys. Rev. Lett. 74, 518-521 (1995).

[12] G. Casati, B. V. Chirikov, D. L. Shepelyansky, Quantum limitations for chaotic excitation of the hydrogen atom in a monochromatic field. Phys. Rev. Lett. 53, 2525-2528 (1984).

[13] G. Casati, B. V. Chirikov, I. Guarneri, D. L. Shepelyansky, Dynamical stability of quantum "chaotic" motion in a hydrogen atom. Phys. Rev. Lett. 56, 2437-2440 (1986).

[14] G. Casati, B. V. Chirikov, D. L. Shepelyansky, I. Guarneri, New photoelectric ionization peak in the hydrogen atom. Phys. Rev. Lett. 57, 823-826 (1986).

[15] G. Casati, B. V. Chirikov, D. L. Shepelyansky, I. Guarneri, Relevance of classical chaos in quantum mechanics: The hydrogen atom in a monochromatic field. Phys. Rep. 154, 77-123 (1987).

[16] G. Casati, B. V. Chirikov, I. Guarneri, D. L. Shepelyansky, Localization of diffusive excitation in the two-dimensional hydrogen atom in a monochromatic field. Phys. Rev. Lett. 59, 2927-2930 (1987).

[17] B. V. Chirikov, F. M. Izrailev, D. L. Shepelyansky, Quantum chaos: Localization vs. ergodicity. Physica D 33, 77-88 (1988).

[18] J. E. Bayfield, G. Casati, I. Guarneri, D. W. Sokol, Localization of classically chaotic diffusion for hydrogen atoms in microwave fields. Phys. Rev. Lett. 63, 364-367 (1989).

[19] B. V. Chirikov, Chaos and Quantum Mechanics, M.-J. Giannoni, A. Voros, J. Zinn-Justin, eds. (Elsevier Sci. Publ., Amsterdam, 1991), vol. 52 of Les Houches Lecture Series, pp. 443-545.

[20] G. Casati, B. V. Chirikov, I. Guarneri, F. M. Izrailev, Band-random-matrix model for quantum localization in conservative systems. Phys. Rev. E 48, R1613-R1616 (1993).

[21] G. Casati, B. V. Chirikov, Quantum chaos: Unexpected complexity. Physica D 86, 220-237 (1995).

[22] G. Casati, B. V. Chirikov, I. Guarneri, F. M. Izrailev, Quantum ergodicity and localization in conservative systems: The Wigner band random matrix model. Phys. Lett. A 223, 430-435 (1996). 
[23] G. Casati, B. V. Chirikov, O. V. Zhirov, Existence of a long time scale in quantum chaos. Phys. Rev. E 55, 7757-7758 (1997).

[24] R. V. Jensen, S. M. Susskind, M. M. Sanders, Chaotic ionization of highly excited hydrogen atoms: Comparison of classical and quantum theory with experiment. Phys. Rep. 201, 1-56 (1991).

[25] P. M. Koch, K. H. A. van Leeuwen, The importance of resonances in microwave "ionization" of excited hydrogen atoms. Phys. Rep. 256, 289-403 (1995).

[26] S. W. Mcdonald, A. N. Kaufman, Spectrum and eigenfunctions for a Hamiltonian with stochastic trajectories. Phys. Rev. Lett. 42, 1189-1191 (1979).

[27] S. W. Mcdonald, A. N. Kaufman, Wave chaos in the stadium - statistical properties of short-wave solutions of the Helmholtz equation. Phys. Rev. A 37, 3067-3086 (1988).

[28] E. J. Heller, Bound-state eigenfunctions of classically chaotic Hamiltonian systems - scars of periodic-orbits. Phys. Rev. Lett. 53, 1515-1518 (1984).

[29] L. Kaplan, E. J. Heller, Linear and nonlinear theory of eigenfunction scars. Ann. Phys. 264, 171-206 (1998).

[30] P. J. J. Luukko, et al., Strong quantum scarring by local impurities. Sci. Rep. 6, 37656 (2016).

[31] S. Fishman, D. R. Grempel, R. E. Prange, Chaos, quantum recurrences, and Anderson localization. Phys. Rev. Lett. 49, 509-512 (1982).

[32] S. Fishman, R. E. Prange, M. Griniasty, Scaling theory for the localization length of the kicked rotor. Phys. Rev. A 39, 1628-1633 (1989).

[33] R. E. Prange, S. Fishman, Experimental realizations of kicked quantum chaotic systems. Phys. Rev. Lett. 63, 704-707 (1989).

[34] R. Blümel, U. Smilansky, Ionization of excited hydrogen atoms by microwave fields: A test case for quantum chaos. Phys. Scrip. 40, 386-393 (1989).

[35] T. Kottos, U. Smilansky, Quantum chaos on graphs. Phys. Rev. Lett. 79, 4794-4797 (1997).

[36] R. Blümel, U. Smilansky, Classical irregular scattering and its quantum-mechanical implications. Phys. Rev. Lett. 60, 477-480 (1988).

[37] R. Blümel, U. Smilansky, A simple-model for chaotic scattering: II. quantum-mechanical theory. Physica D 36, 111-136 (1989).

[38] T. Kottos, U. Smilansky, J. Fortuny, G. Nesti, Chaotic scattering of microwaves. Radio Sci. 34, 747-758 (1999).

[39] T. Kottos, U. Smilansky, Chaotic scattering on graphs. Phys. Rev. Lett. 85, 968-971 (2000).

[40] T. Kottos, U. Smilansky, Quantum graphs: A simple model for chaotic scattering. J. Phys. A Math. Gen. 36, 3501-3524 (2003).

[41] S. Gnutzmann, U. Smilansky, Quantum graphs: Applications to quantum chaos and universal spectral statistics. Adv. Phys. 55, 527-625 (2006).

[42] M. Feingold, S. Fishman, D. R. Grempel, R. E. Prange, Statistics of quasi-energy separations in chaotic systems. Phys. Rev. B 31, 6852-6855 (1985).

[43] M. Feingold, S. Fishman, Statistics of quasienergies in chaotic and random systems. Physica D 25, 181-195 (1987).

[44] O. Agam, S. Fishman, Semiclassical analysis of energy level correlations for a disordered mesoscopic system. Phys. Rev. Lett. 76, 726-729 (1996).

[45] T. Kottos, U. Smilansky, Periodic orbit theory and spectral statistics for quantum graphs. Ann. Phys. 274, 76-124 (1999). 
[46] T. Dittrich, et al., Spectral correlations in systems undergoing a transition from periodicity to disorder. Phys. Rev. E 59, 6541-6551 (1999).

[47] B. Gutkin, U. Smilansky, Can one hear the shape of a graph? J. Phys. A. Math. Gen. 34, 6061-6068 (2001).

[48] S. Rahav, S. Fishman, Spectral statistics of rectangular billiards with localized perturbations. Nonlinearity 15, 1541-1594 (2002).

[49] G. Blum, S. Gnutzmann, U. Smilansky, Nodal domains statistics: A criterion for quantum chaos. Phys. Rev. Lett. 88, 114101 (2002).

[50] S. Gnutzmann, P. D. Karageorge, U. Smilansky, Can one count the shape of a drum? Phys. Rev. Lett. 97, 090201 (2006).

[51] U. Smilansky, Quantum chaos on discrete graphs. J. Phys. A. Math. Gen. 40, F621-F630 (2007).

[52] B. V. Chirikov, An example of chaotic eigenstates in a complex atom. Phys. Lett. A 108, 68-70 (1985).

[53] E. B. Bogomolny, Smoothed wave-functions of chaotic quantum systems. Physica D 31, 169-189 (1988).

[54] R. L. Waterland, J. M. Yuan, C. C. Martens, R. E. Gillilan, W. P. Reinhardt, Classical-quantum correspondence in the presence of global chaos. Phys. Rev. Lett. 61, 2733-2736 (1988).

[55] B. Eckhardt, G. Hose, E. Pollak, Quantum-mechanics of a classically chaotic system - observations on scars, periodic-orbits, and vibrational adiabaticity. Phys. Rev. A 39, 3776-3793 (1989).

[56] R. V. Jensen, M. M. Sanders, M. Saraceno, B. Sundaram, Inhibition of quantum transport due to scars of unstable periodic-orbits. Phys. Rev. Lett. 63, 2771-2775 (1989).

[57] H. J. Stockmann, J. Stein, Quantum chaos in billiards studied by microwave-absorption. Phys. Rev. Lett. 64, 2215-2218 (1990).

[58] B. Eckhardt, J. M. G. Llorente, E. Pollak, Phase-space analysis of chaotic spectra in a conservative Hamiltonian system. Chem. Phys. Lett. 174, 325-332 (1990).

[59] R. Blümel, I. H. Davidson, W. P. Reinhardt, H. Lin, M. Sharnoff, Quasi-linear ridge structures in water-surface waves. Phys. Rev. A 45, 2641-2644 (1992).

[60] M. Kus, J. Zakrzewski, K. Zyczkowski, Quantum scars on a sphere. Phys. Rev. A 43, 4244-4248 (1991).

[61] R. V. Jensen, Quantum chaos. Nature 355, 311-318 (1992).

[62] R. V. Jensen, Quantum mechanics - bringing order out of chaos. Nature 355, 591-592 (1992).

[63] O. Agam, S. Fishman, Quantum eigenfunctions in terms of periodic orbits of chaotic systems. J. Phys. A 26, 2113-2137 (1993).

[64] O. Agam, S. Fishman, Semiclassical criterion for scars in wave functions of chaotic systems. Phys. Rev. Lett. 73, 806-809 (1994).

[65] B. Eckhardt, et al., Approach to ergodicity in quantum wave functions. Phys. Rev. E 52, 5893-5903 (1995).

[66] T. S. Monteiro, D. Delande, J. P. Connerade, Have quantum scars been observed? Nature 387, 863-864 (1997).

[67] C. P. Malta, M. A. M. Deaguiar, A. M. O. Dealmeida, Quantum signature of a period-doubling bifurcation and scars of periodic orbits. Phys. Rev. A 47, 1625-1632 (1993).

[68] G. G. Depolavieja, F. Borondo, R. M. Benito, Scars in groups of eigenstates in a classically chaotic system. Phys. Rev. Lett. 73, 1613-1616 (1994). 
[69] T. M. Fromhold, et al., Manifestations of classical chaos in the energy-level spectrum of a quantumwell. Phys. Rev. Lett. 75, 1142-1145 (1995).

[70] P. Bellomo, T. Uzer, Quantum scars and classical ghosts. Phys. Rev. A 51, 1669-1672 (1995).

[71] O. Agam, Quantum scars of classical orbits in small interacting electronic systems. Phys. Rev. B 54, 2607-2628 (1996).

[72] S. Fishman, B. Georgeot, R. E. Prange, Fredholm method for scars. J. Phys. A 29, 919-937 (1996).

[73] R. Akis, D. K. Ferry, J. P. Bird, Wave function scarring effects in open stadium shaped quantum dots. Phys. Rev. Lett. 79, 123-126 (1997).

[74] F. P. Simonotti, E. Vergini, M. Saraceno, Quantitative study of scars in the boundary section of the stadium billiard. Phys. Rev. E 56, 3859-3867 (1997).

[75] E. E. Narimanov, A. D. Stone, Origin of strong scarring of wave functions in quantum wells in a tilted magnetic field. Phys. Rev. Lett. 80, 49-52 (1998).

[76] L. Kaplan, Scars in quantum chaotic wavefunctions. Nonlinearity 12, R1-R40 (1999).

[77] O. M. Auslaender, S. Fishman, Exact eigenfunctions of a chaotic system. Physica D 128, 180-223 (1999).

[78] J. P. Keating, S. D. Prado, Orbit bifurcations and the scarring of wave functions. Proc. R. Soc. A Math. Phys. Eng. Sci. 457, 1855-1872 (2001).

[79] H. Schanz, T. Kottos, Scars on quantum networks ignore the Lyapunov exponent. Phys. Rev. Lett. 90, 234101 (2003).

[80] R. A. Jalabert., H. U. Baranger, A. D. Stone, Conductance fluctuations in the ballistic regime - a probe of quantum chaos. Phys. Rev. Lett. 65, 2442-2445 (1990).

[81] C. M. Marcus, A. J. Rimberg, R. M. Westervelt, P. F. Hopkins, A. C. Gossard, Conductance fluctuations and chaotic scattering in ballistic microstructures. Phys. Rev. Lett. 69, 506-509 (1992).

[82] Y.-C. Lai, R. Blümel, E. Ott, C. Grebogi, Quantum manifestations of chaotic scattering. Phys. Rev. Lett. 68, 3491-3494 (1992).

[83] R. Ketzmerick, Fractal conductance fluctuations in generic chaotic cavities. Phys. Rev. B 54, 1084110844 (1996).

[84] A. S. Sachrajda, et al., Fractal conductance fluctuations in a soft-wall stadium and a Sinai billiard. Phys. Rev. Lett. 80, 1948-1951 (1998).

[85] B. Huckestein, R. Ketzmerick, C. H. Lewenkopf, Quantum transport through ballistic cavities: Soft vs hard quantum chaos. Phys. Rev. Lett. 84, 5504-5507 (2000).

[86] G. Casati, I. Guarneri, G. Maspero, Fractal survival probability fluctuations. Phys. Rev. Lett. 84, 63-66 (2000).

[87] A. P. S. de Moura, Y.-C. Lai., R. Akis, J. P. Bird, D. K. Ferry, Tunneling and nonhyperbolicity in quantum dots. Phys. Rev. Lett. 88, 236804 (2002).

[88] R. Crook, et al., Imaging fractal conductance fluctuations and scarred wave functions in a quantum billiard. Phys. Rev. Lett. 91, 246803 (2003).

[89] R. Band, A. Sawicki, U. Smilansky, Scattering from isospectral quantum graphs. J. Phys. A. Math. Gen. 43, 415201 (2010).

[90] Y. Krivolapov, S. Fishman, E. Ott, T. M. Antonsen, Quantum chaos of a mixed open system of kicked cold atoms. Phys. Rev. E 83, 016204 (2011).

[91] A. Iomin, S. Fishman, G. M. Zaslavsky, Quantum localization for a kicked rotor with accelerator mode islands. Phys. Rev. E 65, 036215 (2002). 
[92] A. Iomin, S. Fishman, G. M. Zaslavsky, Semiclassical quantization of separatrix maps. Phys. Rev. E 67, 046210 (2003).

[93] M. V. Berry, R. J. Mondragon, Neutrino billiards - time-reversal symmetry-breaking without magnetic-fields. Proc. R. Soc. London Series A Math. Phys. Eng. Sci. 412, 53-74 (1987).

[94] M. Antoine, A. Comtet, M. Knecht, Heat kernel expansion for fermionic billiards in an external magnetic field. J. Phys. A. Math. Gen. 23, L35 (1990).

[95] R. Tomaschitz, Relativistic quantum chaos in Robertson-Walker cosmologies. J. Math. Phys. 32, 2571-2579 (1991).

[96] D. R. Grempel, S. Fishman, R. E. Prange, Localization in an incommensurate potential: An exactly solvable model. Phys. Rev. Lett. 49, 833-836 (1982).

[97] R. E. Prange, D. R. Grempel, S. Fishman, Solvable model of quantum motion in an incommensurate potential. Phys. Rev. B 29, 6500-6512 (1984).

[98] M. V. Berry, Incommensurability in an exactly-soluble quantal and classical model for a kicked rotator. Physica D 10, 369-378 (1984).

[99] A. H. C. Neto, K. Novoselov, Two-dimensional crystals: Beyond graphene. Mater. Exp. 1, 10-17 (2011).

[100] P. Ajayan, P. Kim, K. Banerjee, Two-dimensional van der Waals materials. Phys. Today 69, 38-44 (2016).

[101] K. S. Novoselov, et al., Electric field effect in atomically thin carbon films. Science 306, 666-669 (2004).

[102] C. Berger, et al., Ultrathin epitaxial graphite: 2D electron gas properties and a route toward graphenebased nanoelectronics. J. Phys. Chem. B 108, 19912-19916 (2004).

[103] K. S. Novoselov, et al., Two-dimensional gas of massless Dirac fermions in graphene. Nature 438, 197-200 (2005).

[104] Y. B. Zhang, Y. W. Tan, H. L. Stormer, P. Kim, Experimental observation of the quantum Hall effect and Berry's phase in graphene. Nature 438, 201-204 (2005).

[105] A. H. Castro Neto, F. Guinea, N. M. R. Peres, K. S. Novoselov, A. K. Geim, The electronic properties of graphene. Rev. Mod. Phys. 81, 109-162 (2009).

[106] N. M. R. Peres, Colloquium: The transport properties of graphene: An introduction. Rev. Mod. Phys. 82, 2673-2700 (2010).

[107] S. D. Sarma, S. Adam, E. H. Hwang, E. Rossi, Electronic transport in two-dimensional graphene. Rev. Mod. Phy. 83, 407-470 (2011).

[108] M. Z. Hasan, C. L. Kane, Colloquium: Topological insulators. Rev. Mod. Phys. 82, 3045-3067 (2010).

[109] X.-L. Qi, S.-C. Zhang, Topological insulators and superconductors. Rev. Mod. Phys. 83, 1057-1110 (2011).

[110] B. Radisavljevic, A. Radenovic, J. Brivio, V. Giacometti, A. Kis, Single-layer $\mathrm{MoS}_{2}$ transistors. Nat. Nanotech. 6, 147-150 (2011).

[111] Q. H. Wang, K. Kalantar-Zadeh, A. Kis, J. N. Coleman, M. S. Strano, Electronics and optoelectronics of two-dimensional transition metal dichalcogenides. Nat. Nanotech. 7, 699-712 (2012).

[112] D. Sheberla, et al., High electrical conductivity in $\mathrm{Ni}_{3}(2,3,6,7,10,11 \text {-hexaiminotriphenylene })_{2}$ - a semiconducting metal organic graphene analogue. J. Am. Chem. Soc. 136, 8859-8862 (2014).

[113] Z. K. Liu, et al., Discovery of a three-dimensional topological Dirac semimetal $\mathrm{Na}_{3} \mathrm{Bi}$. Science 343, 864-867 (2014). 
[114] Z. K. Liu, et al., A stable three-dimensional topological Dirac semimetal $\mathrm{Cd}_{3} \mathrm{As}_{2}$. Nat. Mater. 13, 677-681 (2014).

[115] L. Huang, Y.-C. Lai, C. Grebogi, Relativistic quantum level-spacing statistics in chaotic graphene billiards. Phys. Rev. E 81, 055203 (2010).

[116] M. Wimmer, A. R. Akhmerov, F. Guinea, Robustness of edge states in graphene quantum dots. Phys. Rev. B 82, 045409 (2010).

[117] K. Wakabayashi, S. Okada, R. Tomita, S. Fujimoto, Y. Natsume, Edge states and flat bands of graphene nanoribbons with edge modification. J. Phys. Soc. Jpn. 79, 034706 (2010).

[118] L. Huang, Y.-C. Lai, C. Grebogi, Characteristics of level-spacing statistics in chaotic graphene billiards. Chaos 21, 013102 (2011).

[119] A. Rycerz, Random matrices and quantum chaos in weakly disordered graphene nanoflakes. Phys. Rev. B 85, 245424 (2012).

[120] P. Yu, et al., Gaussian orthogonal ensemble statistics in graphene billiards with the shape of classically integrable billiards. Phys. Rev. E 94, 062214 (2016).

[121] L. Huang, Y.-C. Lai, D. K. Ferry, S. M. Goodnick, R. Akis, Relativistic quantum scars. Phys. Rev. Lett. 103, 054101 (2009).

[122] X. Ni, L. Huang, Y.-C. Lai, C. Grebogi, Scarring of Dirac fermions in chaotic billiards. Phys. Rev. E 86, 016702 (2012).

[123] H. Y. Xu, L. Huang, Y.-C. Lai, C. Grebogi, Chiral scars in chaotic Dirac fermion systems. Phys. Rev. Lett. 110, 064102 (2013).

[124] C.-Z. Wang, L. Huang, K. Chang, Scars in Dirac fermion systems: The influence of an AharonovBohm flux. New J. Phys. 19, 013018 (2017).

[125] R. Yang, L. Huang, Y.-C. Lai, C. Grebogi, Quantum chaotic scattering in graphene systems. Europhys. Lett. 94, 40004 (2011).

[126] R. Yang, L. Huang, Y.-C. Lai, C. Grebogi, Abnormal electron paths induced by Klein tunneling in graphene quantum point contacts. Phys. Rev. B 84, 035426 (2011).

[127] R. Yang, L. Huang, Y.-C. Lai, L. M. Pecora, Modulating quantum transport by transient chaos. Appl. Phys. Lett. 100, 093105 (2012).

[128] R. Yang, L. Huang, Y.-C. Lai, C. Grebogi, L. M. Pecora, Harnessing quantum transport by transient chaos. Chaos 23, 013125 (2013).

[129] M. S. M. Barros, A. J. N. Júnior, A. F. Macedo-Junior, J. G. G. S. Ramos, A. L. R. Barbosa, Open chaotic Dirac billiards: Weak (anti)localization, conductance fluctuations, and decoherence. Phys. Rev. B 88, 245133 (2013).

[130] G. L. Wang, L. Ying, Y.-C. Lai, C. Grebogi, Quantum chaotic scattering in graphene systems in the absence of invariant classical dynamics. Phys. Rev. E 87, 052908 (2013).

[131] J. G. G. S. Ramos, I. M. L. da Silva, A. L. R. Barbosa, Anomalous entanglement in chaotic Dirac billiards. Phys. Rev. B 90, 245107 (2014).

[132] J. G. G. S. Ramos, M. S. Hussein, A. L. R. Barbosa, Fluctuation phenomena in chaotic Dirac quantum dots: Artificial atoms on graphene flakes. Phys. Rev. B 93, 125136 (2016).

[133] T. C. Vasconcelos, J. G. G. S. Ramos, A. L. R. Barbosa, Universal spin Hall conductance fluctuations in chaotic Dirac quantum dots. Phys. Rev. B 93, 115120 (2016).

[134] X. Ni, L. Huang, Y.-C. Lai, L. M. Pecora, Effect of chaos on relativistic quantum tunneling. Europhys. Lett. 98, 50007 (2012). 
[135] X. Ni, L. Huang, L. Ying, Y.-C. Lai, Relativistic quantum tunneling of a Dirac fermion in nonhyperbolic chaotic systems. Phys. Rev. B 87, 224304 (2013).

[136] L. Ying, G. Wang, L. Huang, Y.-C. Lai, Quantum chaotic tunneling in graphene systems with electron-electron interactions. Phys. Rev. B 90, 224301 (2014).

[137] H.-Y. Xu, L. Huang, Y.-C. Lai, C. Grebogi, Superpersistent currents and whispering gallery modes in relativistic quantum chaotic systems. Sci. Rep. 5, 8963 (2015).

[138] H.-Y. Xu, L. Huang, Y.-C. Lai, A robust relativistic quantum two-level system with edge-dependent currents and spin polarization. Europhys. Lett. 115, 20005 (2016).

[139] L. Ying, Y.-C. Lai, Robustness of persistent currents in two-dimensional Dirac systems with disorder. Phys. Rev. B 96, 165407 (2017).

[140] J. H. Bardarson, M. Titov, P. W. Brouwer, Electrostatic confinement of electrons in an integrable graphene quantum dot. Phys. Rev. Lett. 102, 226803 (2009).

[141] M. Schneider, P. W. Brouwer, Resonant scattering in graphene with a gate-defined chaotic quantum dot. Phys. Rev. B 84, 115440 (2011).

[142] J. Heinl, M. Schneider, P. W. Brouwer, Interplay of Aharonov-Bohm and Berry phases in gate-defined graphene quantum dots. Phys. Rev. B 87, 245426 (2013).

[143] M. Schneider, P. W. Brouwer, Density of states as a probe of electrostatic confinement in graphene. Phys. Rev. B 89, 205437 (2014).

[144] L. Ying, Y.-C. Lai, Enhancement of spin polarization by chaos in graphene quantum dot systems. Phys. Rev. B 93, 085408 (2016).

[145] I. Hagymási, P. Vancsó, A. Pálinkás, Z. Osváth, Interaction effects in a chaotic graphene quantum billiard. Phys. Rev. B 95, 075123 (2017).

[146] L. L. Jiang, L. Huang, R. Yang, Y.-C. Lai, Control of transmission in disordered graphene nanojunctions through stochastic resonance. Appl. Phys. Lett. 96, 262114 (2010).

[147] L. Ying, L. Huang, Y.-C. Lai, C. Grebogi, Conductance fluctuations in graphene systems: The relevance of classical dynamics. Phys. Rev. B 85, 245448 (2012).

[148] L. Huang, R. Yang, Y.-C. Lai, D. K. Ferry, Lead-position dependent regular oscillations and random fluctuations of conductance in graphene quantum dots. J. Phys. Cond. Mat. 25, 085502 (2013).

[149] L. Ying, L. Huang, Y.-C. Lai, Y. Zhang, Effect of geometrical rotation on conductance fluctuations in graphene quantum dots. J. Phys. Cond. Mat. 25, 105802 (2013).

[150] H. Xu, Y.-C. Lai, Reverse Stark effect, anomalous optical transitions, and control of spin in topological insulator quantum dots. Phys. Rev. B 92, 195120 (2015).

[151] M. Robnik, Quantizing a generic family of billiards with analytic boundaries. J. Phys. A Math. Gen. 17, 1049 (1984).

[152] M. C. Gutzwiller, Chaos in Classical and Quantum Mechanics (Springer, New York, 2013).

[153] H. A. Weidenmüller, G. E. Mitchell, Random matrices and chaos in nuclear physics: Nuclear structure. Rev. Mod. Phys. 81, 539-589 (2009).

[154] P. Strange, Relativistic Quantum Mechanics: with Applications in Condensed Matter and Atomic Physics (Cambridge University Press, Cambridge UK, 1998).

[155] P. Blanchard, R. Olkiewicz, Decoherence-induced continuous pointer states. Phys. Rev. Lett. 90, 010403 (2003).

[156] W. H. Zurek, Decoherence, einselection, and the quantum origins of the classical. Rev. Mod. Phys. 75, 715-775 (2003). 
[157] D. K. Ferry, R. Akis, J. P. Bird, Einselection in action: Decoherence and pointer states in open quantum dots. Phys. Rev. Lett. 93, 026803 (2004).

[158] R. Brunner, R. Akis, D. K. Ferry, F. Kuchar, R. Meisels, Coupling-induced bipartite pointer states in arrays of electron billiards: Quantum Darwinism in action? Phys. Rev. Lett. 101, 024102 (2008).

[159] D. K. Ferry, L. Huang, R. Yang, Y.-C. Lai, R. Akis, Open quantum dots in graphene: Scaling relativistic pointer states. J. Phys. Conf. Ser. 220, 012015 (2010).

[160] M. Zwolak, W. H. Zurek, Complementarity of quantum discord and classically accessible information. Sci. Rep. 3, 1729 (2013).

[161] H. U. Baranger, R. A. Jalabert, A. D. Stone, Weak localization and integrability in ballistic cavities. Phys. Rev. Lett. 70, 3876-3879 (1993).

[162] T. M. Fromhold, et al., Magnetotunneling spectroscopy of a quantum well in the regime of classical chaos. Phys. Rev. Lett. 72, 2608-2611 (1994).

[163] L. Huang, Y.-C. Lai, H.-G. Luo, C. Grebogi, Universal formalism of Fano resonance. AIP Adv. 5, 017137 (2015).

[164] S. Bleher, C. Grebogi, E. Ott, Bifurcation to chaotic scattering. Physica D 45, 87-121 (1990).

[165] M. Ding, C. Grebogi, E. Ott, J. A. Yorke, Transition to chaotic scattering. Phys. Rev. A 42, 7025-7040 (1990).

[166] E. Ott, T. Tél, Focus issue on chaotic scattering. Chaos 3, 417-706 (1993).

[167] Y.-C. Lai, C. Grebogi, R. Blümel, I. Kan, Crisis in chaotic scattering. Phys. Rev. Lett. 71, 2212-2215 (1993).

[168] Y.-C. Lai, C. Grebogi, Crisis and enhancement of chaotic scattering. Phys. Rev. E 49, 3761-3770 (1994).

[169] Y.-C. Lai, Abrupt bifurcation to chaotic scattering with discontinuous change in fractal dimension. Phys. Rev. E 60, R6283-R6286 (1999).

[170] P. Gaspard, Chaos, Scattering and Statistical Mechanics (Cambridge University Press, Cambridge, UK, 1998), first edn.

[171] Y.-C. Lai, T. Tél, Transient Chaos: Complex Dynamics on Finite-Time Scales (Springer, New York, 2011).

[172] W. H. Miller, The classical S-matrix in molecular collisions. Advances in Chemical Physics 30, 77-136 (1975).

[173] R. Blümel, U. Smilansky, A simple model for chaotic scattering: II. quantum mechanical theory. Physica D 36, 111-136 (1989).

[174] S. Tomsovic, D. Ullmo, Chaos-assisted tunneling. Phys. Rev. E 50, 145-162 (1994).

[175] F. Leyvraz, D. Ullmo, The level splitting distribution in chaos-assisted tunnelling. J. Phys. Math. Gen. 29, 2529-2551 (1996).

[176] C. Dembowski, et al., First experimental evidence for chaos-assisted tunneling in a microwave annular billiard. Phys. Rev. Lett. 84, 867-870 (2000).

[177] S. Tomsovic, Tunneling and chaos. Phys. Scrip. T90, 162-165 (2001).

[178] D. A. Steck, W. H. Oskay, M. G. Raizen, Observation of chaos-assisted tunneling between islands of stability. Science 293, 274-278 (2001).

[179] A. Bäcker, R. Ketzmerick, S. Löck, L. Schilling, Regular-to-chaotic tunneling rates using a fictitious integrable system. Phys. Rev. Lett. 100, 104101 (2008).

[180] L. M. Pecora, et al., Chaos regularization of quantum tunneling rates. Phys. Rev. E 83, 065201 (2011). 
[181] M. J. Lee, T. M. Antonsen, E. Ott, L. M. Pecora, Theory of chaos regularization of tunneling in chaotic quantum dots. Phys. Rev. E 86, 056212 (2012).

[182] M. Büttiker, Y. Imry, R. Landauer, Josephson behavior in small normal one-dimensional rings. Phys. Lett. A 96, 365-367 (1983).

[183] Y. Aharonov, D. Bohm, Significance of electromagnetic potentials in the quantum theory. Phys. Rev. 115, 485 (1959).

[184] L. P. Lévy, G. Dolan, J. Dunsmuir, H. Bouchiat, Magnetization of mesoscopic copper rings: Evidence for persistent currents. Phys. Rev. Lett. 64, 2074-2077 (1990).

[185] V. Chandrasekhar, et al., Magnetic response of a single, isolated gold loop. Phys. Rev. Lett. 67, 3578-3581 (1991).

[186] A. C. Bleszynski-Jayich1, et al., Persistent currents in normal metal rings. Science 326, 272-275 (2009).

[187] H. Bluhm, N. C. Koshnick, J. A. Bert, M. E. Huber, K. A. Moler, Persistent currents in normal metal rings. Phys. Rev. Lett. 102, 136802 (2009).

[188] M. A. Castellanos-Beltran, D. Q. Ngo, W. E. Shanks, A. B. Jayich, J. G. E. Harris, Measurement of the full distribution of persistent current in normal-metal rings. Phys. Rev. Lett. 110, 156801 (2013).

[189] D. Mailly, C. Chapelier, A. Benoit, Experimental observation of persistent currents in GaAs-AlGaAs single loop. Phys. Rev. Lett. 70, 2020-2023 (1993).

[190] W. Rabaud, et al., Persistent currents in mesoscopic connected rings. Phys. Rev. Lett. 86, 3124-3127 (2001).

[191] N. A. J. M. Kleemans, et al., Oscillatory persistent currents in self-assembled quantum rings. Phys. Rev. Lett. 99, 146808 (2007).

[192] D. Berman, O. Entin-Wohlman, M. Y. Azbel, Diamagnetic spectrum and oscillations in an elliptic shell. Phys. Rev. B 42, 9299-9306 (1990).

[193] T. Chakraborty, P. Pietiläinen, Persistent currents in a quantum ring: Effects of impurities and interactions. Phys. Rev. B 52, 1932-1935 (1995).

[194] D. V. Bulaev, V. A. Geyler, V. A. Margulis, Effect of surface curvature on magnetic moment and persistent currents in two-dimensional quantum rings and dots. Phys. Rev. B 69, 195313 (2004).

[195] Y. V. Pershin, C. Piermarocchi, Persistent and radiation-induced currents in distorted quantum rings. Phys. Rev. B 72, 125348 (2005).

[196] A. Bruno-Alfonso, A. Latgé, Aharonov-Bohm oscillations in a quantum ring: Eccentricity and electric-field effects. Phys. Rev. B 71, 125312 (2005).

[197] A. Bruno-Alfonso, A. Latgé, Quantum rings of arbitrary shape and non-uniform width in a threading magnetic field. Phys. Rev. B 77, 205303 (2008).

[198] A. Fuhrer, et al., Energy spectra of quantum rings. Nature 413, 822-825 (2001).

[199] S. Reich, J. Maultzsch, C. Thomsen, P. Ordejón, Tight-binding description of graphene. Phys. Rev. $B$ 66, 035412 (2002).

[200] A. R. Akhmerov, C. W. J. Beenakker, Boundary conditions for Dirac fermions on a terminated honeycomb lattice. Phys. Rev. B 77, 085423 (2008).

[201] M. Titov, P. M. Ostrovsky, I. V. Gornyi, A. Schuessler, A. D. Mirlin, Charge transport in graphene with resonant scatterers. Phys. Rev. Lett. 104, 076802 (2010).

[202] K.-H. Ahn, K. Richter, I.-H. Lee, Addition spectra of chaotic quantum dots: Interplay between interactions and geometry. Phys. Rev. Lett. 83, 4144-4147 (1999). 
[203] R. Shankar, Colloquium: Chaotic quantum dots with strongly correlated electrons. Rev. Mod. Phys. 80, 379-394 (2008).

[204] D. Ullmo, Many-body physics and quantum chaos. Rep. Prog. Phys. 81, 026001 (2008).

[205] J. M. G. Gómeza, et al., Many-body quantum chaos: Recent developments and applications to nuclei. Phys. Rep. 499, 103-226 (2011).

[206] J. Hubbard, Electron correlations in narrow energy bands. Proc. R. Soc. London Series A Math. Phys. Sci. 276, 238-257 (1963).

[207] O. V. Yazyev, Emergence of magnetism in graphene materials and nanostructures. Rep. Prog. Phys. 73, 056501 (2010).

[208] M. Fujita, K. Wakabayashi, K. Nakada, K. Kusakabe, Peculiar localized state at zigzag graphite edge. J. Phys. Soc. Jpn. 65, 1920-1923 (1996).

[209] J. Fernandez-Rossier, J. J. Palacios, Magnetism in graphene nanoislands. Phys. Rev. Lett. 99, 177204 (2007).

[210] F. Liu, Y. L. Li, X. Y. Liu, J. F. Kang, Gate voltage induced spin-filtering effect in a junction based on zigzag graphene nanoribbons. Appl. Phys. Lett. 99, 243503 (2011).

[211] Y. C. Chang, S. Haas, Defect-induced resonances and magnetic patterns in graphene. Phys. Rev. $B$ 83, 085406 (2011).

[212] Y. Hancock, A. Uppstu, K. Saloriutta, A. Harju, M. J. Puska, Generalized tight-binding transport model for graphene nanoribbon-based systems. Phys. Rev. B 81, 245402 (2010).

[213] H. Feldner, et al., Magnetism of finite graphene samples: Mean-field theory compared with exact diagonalization and quantum Monte Carlo simulations. Phys. Rev. B 81, 115416 (2010).

[214] K. Wakabayashi, M. Sigrist, M. Fujita, Spin wave mode of edge-localized magnetic states in nanographite zigzag ribbons. J. Phys. Soc. Jpn. 67, 2089-2093 (1998).

[215] H. Feldner, et al., Dynamical signatures of edge-state magnetism on graphene nanoribbons. Phys. Rev. Lett. 106, 226401 (2011).

[216] M. Polini, F. Guinea, M. Lewenstein, H. C. Manoharan, V. Pellegrini, Artificial honeycomb lattices for electrons, atoms and photons. Nat. Nanotech. 8, 625-633 (2013).

[217] S. Bittner, et al., Observation of a Dirac point in microwave experiments with a photonic crystal modeling graphene. Phys. Rev. B 82, 014301 (2010).

[218] U. Kuhl, et al., Dirac point and edge states in a microwave realization of tight-binding graphene-like structures. Phys. Rev. B 82, 094308 (2010).

[219] M. Bellec, U. Kuhl, G. Montambaux, F. Mortessagne, Topological transition of Dirac points in a microwave experiment. Phys. Rev. Lett. 110, 033902 (2013).

[220] S. Bittner, B. Dietz, M. Miski-Oglu, A. Richter, Extremal transmission through a microwave photonic crystal and the observation of edge states in a rectangular Dirac billiard. Phys. Rev. B 85, 064301 (2012).

[221] M. Bellec, U. Kuhl, G. Montambaux, F. Mortessagne, Tight-binding couplings in microwave artificial graphene. Phys. Rev. B 88, 115437 (2013).

[222] X. Wang, et al., Anomalous transmission of disordered photonic graphenes at the Dirac point. Europhys. Lett. 103, 17003 (2013).

[223] X. Wang, et al., Transmission properties near Dirac-like point in two-dimensional dielectric photonic crystals. Europhys. Lett. 108, 14002 (2014).

[224] B. Dietz, T. Klaus, M. Miski-Oglu, A. Richter, Spectral properties of superconducting microwave photonic crystals modeling Dirac billiards. Phys. Rev. B 91, 035411 (2015). 
[225] B. Dietz, A. Richter, Quantum and wave dynamical chaos in superconducting microwave billiards. Chaos 25, 097601 (2015).

[226] X. Wang, et al., Transport properties of disordered photonic crystals around a Dirac-like point. Opt. Expr. 23, 5126-5133 (2015).

[227] B. Dietz, et al., Spectral properties of Dirac billiards at the van Hove singularities. Phys. Rev. Lett. 116, 023901 (2016).

[228] S. R. Zandbergen, M. J. A. de Dood, Experimental observation of strong edge effects on the pseudodiffusive transport of light in photonic graphene. Phys. Rev. Lett. 104, 043903 (2010).

[229] Y. Poo, R.-X. Wu, Z. Lin, Y. Yang, C. T. Chan, Experimental realization of self-guiding unidirectional electromagnetic edge states. Phys. Rev. Lett. 106, 093903 (2011).

[230] Y. Plotnik, et al., Observation of unconventional edge states in photonic graphene. Nat. Mater. 13, 57-62 (2013).

[231] Y. Xiong, Dynamics of Dirac quasi-particles in lattice vibration and anomalous phonon frequency shift of graphene. Solid State Commun. 151, 785-789 (2011).

[232] D. Torrent, J. Sánchez-Dehesa, Acoustic analogue of graphene: Observation of Dirac cones in acoustic surface waves. Phys. Rev. Lett. 108, 174301 (2012).

[233] J. Lu, et al., Dirac cones in two-dimensional artificial crystals for classical waves. Phys. Rev. B 89, 134302 (2014).

[234] F. Iachello, B. Dietz, M. Miski-Oglu, A. Richter, Algebraic theory of crystal vibrations: Singularities and zeros in vibrations of one- and two-dimensional lattices. Phys. Rev. B 91, 214307 (2015).

[235] S. Yu, et al., Surface phononic graphene. Nat. Mater. 15, 1243-1247 (2016).

[236] C. He, et al., Acoustic topological insulator and robust one-way sound transport. Nat. Phys. 12, 1124-1129 (2016).

[237] P. R. Wallace, The band theory of graphite. Phys. Rev. 71, 622-634 (1947).

[238] V. Alonso, S. De Vincenzo, General boundary conditions for a Dirac particle in a box and their non-relativistic limits. J. Phys. A Math. Gen. 30, 8573 (1997).

[239] P. Alberto, C. Fiolhais, V. Gil, Relativistic particle in a box. Eur. J. Phys. 17, 19 (1996).

[240] V. Alonso, S. De Vincenzo, L. Mondino, On the boundary conditions for the Dirac equation. Eur. J. Phys. 18, 315 (1997).

[241] A. Chodos, R. L. Jaffe, K. Johnson, C. B. Thorn, V. F. Weisskopf, New extended model of hadrons. Phys. Rev. D 9, 3471-3495 (1974).

[242] A. Chodos, R. L. Jaffe, K. Johnson, C. B. Thorn, Baryon structure in the bag theory. Phys. Rev. D 10, 2599-2604 (1974).

[243] A. W. Thomas, Chiral symmetry and the bag model: A new starting point for nuclear physics. Adv. Nucl. Phys. 13, 1-137 (1984).

[244] M. E. Peskin, D. V. Schroeder, An Introduction to Quantum Field Theory (Westview Press, 1996).

[245] R. Stacey, Eliminating lattice fermion doubling. Phys. Rev. D 26, 468-472 (1982).

[246] C. M. Bender, K. A. Milton, D. H. Sharp, Consistent formulation of fermions on a Minkowski lattice. Phys. Rev. Lett. 51, 1815-1818 (1983).

[247] M. V. Berry, M. Wilkinson, Proc. R. Soc. London Series A Math. Phys. Eng. Sci. (The Royal Society, 1984), vol. 392, pp. 15-43.

[248] R. J. Riddell, Boundary-distribution solution of the Helmholtz equation for a region with corners. $J$. Comp. Phys. 31, 21-41 (1979). 
[249] B. Li, M. Robnik, B. Hu, Relevance of chaos in numerical solutions of quantum billiards. Phys. Rev. E 57, 4095-4105 (1998).

[250] J. Cserti, A. Csordás, U. Zülicke, Electronic and spin properties of Rashba billiards. Phys. Rev. B 70, 233307 (2004).

[251] P. Recher, et al., Aharonov-Bohm effect and broken valley degeneracy in graphene rings. Phys. Rev. $B$ 76, 235404 (2007).

[252] S. Leseduarte, A. Romeo, Influence of a magnetic fluxon on the vacuum energy of quantum fields confined by a bag. Commun. Math. Phys. 193, 317-336 (1998).

[253] C. G. Beneventano, M. De Francia, K. Kirsten, E. M. Santangelo, Casimir energy of massive mit fermions in an Aharonov-Bohm background. Phys. Rev. D 61, 085019 (2000).

[254] M. Robnik, Classical dynamics of a family of billiards with analytic boundaries. J. Phys. A 16, 3971 (1983).

[255] H. Bruus, A. D. Stone, Quantum chaos in a deformable billiard: Applications to quantum dots. Phys. Rev. B 50, 18275 (1994).

[256] M. V. Berry, M. Robnik, Statistics of energy levels without time-reversal symmetry: Aharonov-Bohm chaotic billiards. J. Phys. A 19, 649 (1986).

[257] U. Stoffregen, J. Stein, H.-J. Stöckmann, M. Kuś, F. Haake, Microwave billiards with broken time reversal symmetry. Phys. Rev. Lett. 74, 2666-2669 (1995).

[258] P. So, S. M. Anlage, E. Ott, R. N. Oerter, Wave chaos experiments with and without time reversal symmetry: GUE and GOE statistics. Phys. Rev. Lett. 74, 2662-2665 (1995).

[259] C. Dembowski, et al., Gaussian unitary ensemble statistics in a time-reversal invariant microwave triangular billiard. Phys. Rev. E 62, R4516-R4519 (2000).

[260] C. Dembowski, et al., Phase shift experiments identifying kramers doublets in a chaotic superconducting microwave billiard of threefold symmetry. Phys. Rev. Lett. 90, 014102 (2003).

[261] M. Aßmann, J. Thewes, D. Fröhlich, M. Bayer, Quantum chaos and breaking of all anti-unitary symmetries in Rydberg excitons. Nat. Mater. 15, 741-745 (2016).

[262] O. Hul, et al., Experimental simulation of quantum graphs by microwave networks. Phys. Rev. E 69, 056205 (2004).

[263] M. Białous, et al., Power spectrum analysis and missing level statistics of microwave graphs with violated time reversal invariance. Phys. Rev. Lett. 117, 144101 (2016).

[264] A. Rehemanjiang, et al., Microwave realization of the Gaussian symplectic ensemble. Phys. Rev. Lett. 117, 064101 (2016).

[265] M. Katsnelson, K. Novoselov, A. Geim, Chiral tunnelling and the Klein paradox in graphene. Nat. Phys. 2, 620-625 (2006).

[266] C. W. J. Beenakker, Colloquium: Andreev reflection and Klein tunneling in graphene. Rev. Mod. Phys. 80, 1337-1354 (2008).

[267] G. Karagiorgi, et al., Leptonic $C P$ violation studies at miniboone in the $(3+2)$ sterile neutrino oscillation hypothesis. Phys. Rev. D 75, 013011 (2007).

[268] S. C. Phatak, S. Pal, D. Biswas, Semiclassical features in the quantum description of a Dirac particle in a cavity. Phys. Rev. E 52, 1333-1344 (1995).

[269] A. R. Akhmerov, C. W. J. Beenakker, Detection of valley polarization in graphene by a superconducting contact. Phys. Rev. Lett. 98, 157003 (2007).

[270] D. Xiao, W. Yao, Q. Niu, Valley-contrasting physics in graphene: Magnetic moment and topological transport. Phys. Rev. Lett. 99, 236809 (2007). 
[271] J. L. Garcia-Pomar, A. Cortijo, M. Nieto-Vesperinas, Fully valley-polarized electron beams in graphene. Phys. Rev. Lett. 100, 236801 (2008).

[272] F. Miao, et al., Phase-coherent transport in graphene quantum billiards. Science 317, 1530-1533 (2007).

[273] L. Ponomarenko, et al., Chaotic Dirac billiard in graphene quantum dots. Science 320, 356-358 (2008).

[274] C. Stampfer, et al., Tunable graphene single electron transistor. Nano Lett. 8, 2378-2383 (2008).

[275] C.-L. Tan, et al., Quantum chaos in graphene nanoribbon quantum dot. Acta Physica Sinica 58, 5726 (2009).

[276] M. V. Berry, M. Tabor, Level clustering in the regular spectrum. Proc. Royal Soc. A 356, 375-394 (1977).

[277] M. L. Mehta, Random matrices, vol. 142 (Academic press, 2004).

[278] M. L. Mehta, F. J. Dyson, Statistical theory of the energy levels of complex systems. V. J. Math. Phys. 4, 713-719 (1963).

[279] O. Bohigas, M. Giannoni, Level density fluctuations and random matrix theory. Ann. Phys. 89, 393-422 (1975).

[280] B. Liu, G.-C. Zhang, L. Ding, J.-H. Dai, H.-J. Zhang, The energy spectral analysis of the strongcoupled superlattice. Phys. Lett. A 260, 406-410 (1999).

[281] H. Hasegawa, H. J. Mikeska, H. Frahm, Stochastic formulation of energy-level statistics. Phys. Rev. A 38, 395-399 (1988).

[282] L. Huang, H.-Y. Xu, Y.-C. Lai, C. Grebogi, Level spacing statistics for two-dimensional massless Dirac billiards. Chin. Phys. B 23, 070507 (2014).

[283] R. Saito, G. Dresselhaus, M. S. Dresselhaus, Trigonal warping effect of carbon nanotubes. Phys. Rev. B 61, 2981-2990 (2000).

[284] A. Rycerz, J. Tworzydło, C. W. J. Beenakker, Valley filter and valley valve in graphene. Nat. Phys. 3, 172-175 (2007).

[285] V. V. Cheianov, V. Fal'ko, B. L. Altshuler, The focusing of electron flow and a Veselago lens in graphene p-n junctions. Science 315, 1252-1255 (2007).

[286] F. Libisch, C. Stampfer, J. Burgdörfer, Graphene quantum dots: Beyond a Dirac billiard. Phys. Rev. $B$ 79, 115423 (2009).

[287] I. Amanatidis, S. N. Evangelou, Quantum chaos in weakly disordered graphene. Phys. Rev. B 79, 205420 (2009).

[288] E. Amanatidis, I. Kleftogiannis, D. Katsanos, S. Evangelou, Critical level statistics for weakly disordered graphene. J. Phys. Cond. Mat. 26, 155601 (2014).

[289] Y.-W. Son, M. L. Cohen, S. G. Louie, Energy gaps in graphene nanoribbons. Phys. Rev. Lett. 97, 216803 (2006).

[290] C. Lanczos, An iteration method for the solution of the eigenvalue problem of linear differential and integral operators. J. Res. Natl. Bur. Stand. 45, 255 (1950).

[291] J.-H. Chen, et al., Charged-impurity scattering in graphene. Nat. Phys. 4, 377-381 (2008).

[292] L. Brey, H. A. Fertig, Electronic states of graphene nanoribbons studied with the Dirac equation. Phys. Rev. B 73, 235411 (2006).

[293] H. Suzuura, T. Ando, Crossover from symplectic to orthogonal class in a two-dimensional honeycomb lattice. Phys. Rev. Lett. 89, 266603 (2002). 
[294] Y. Alhassid, C. H. Lewenkopf, Statistical distributions of level widths and conductance peaks in irregularly shaped quantum dots. Phys. Rev. Lett. 75, 3922-3925 (1995).

[295] M. Robnik, M. V. Berry, Classical billiards in magnetic fields. J. Phys. A 18, 1361 (1985).

[296] M. Robnik, Perimeter corrections to the Landau diamagnetism. J. Phys. A 19, 3619 (1986).

[297] R. Bell, P. Dean, Atomic vibrations in vitreous silica. Discuss. Faraday Soc. 50, 55-61 (1970).

[298] R. Bell, The dynamics of disordered lattices. Rep. Prog. Phys. 35, 1315 (1972).

[299] J. Wurm, et al., Symmetry classes in graphene quantum dots: Universal spectral statistics, weak localization, and conductance fluctuations. Phys. Rev. Lett. 102, 056806 (2009).

[300] M. Fujita, K. Wakabayashi, K. Nakada, K. Kusakabe, Peculiar localized state at zigzag graphite edge. J. Phys. Soc. Jpn. 65, 1920-1923 (1996).

[301] M. Wimmer, I. Adagideli, S. Berber, D. Tománek, K. Richter, Spin currents in rough graphene nanoribbons: Universal fluctuations and spin injection. Phys. Rev. Lett. 100, 177207 (2008).

[302] S. Ryu, C. Mudry, H. Obuse, A. Furusaki, $Z_{2}$ topological term, the global anomaly, and the twodimensional symplectic symmetry class of Anderson localization. Phys. Rev. Lett. 99, 116601 (2007).

[303] K. Nomura, M. Koshino, S. Ryu, Topological delocalization of two-dimensional massless Dirac fermions. Phys. Rev. Lett. 99, 146806 (2007).

[304] P. M. Ostrovsky, I. V. Gornyi, A. D. Mirlin, Quantum criticality and minimal conductivity in graphene with long-range disorder. Phys. Rev. Lett. 98, 256801 (2007).

[305] C. Bena, Effect of a single localized impurity on the local density of states in monolayer and bilayer graphene. Phys. Rev. Lett. 100, 076601 (2008).

[306] I. Brihuega, et al., Quasiparticle chirality in epitaxial graphene probed at the nanometer scale. Phys. Rev. Lett. 101, 206802 (2008).

[307] T. Ando, T. Nakanishi, R. Saito, Berry's phase and absence of back scattering in carbon nanotubes. J. Phys. Soc. Jpn. 67, 2857-2862 (1998).

[308] S. V. Morozov, et al., Strong suppression of weak localization in graphene. Phys. Rev. Lett. 97, 016801 (2006).

[309] E. McCann, et al., Weak-localization magnetoresistance and valley symmetry in graphene. Phys. Rev. Lett. 97, 146805 (2006).

[310] A. F. Morpurgo, F. Guinea, Intervalley scattering, long-range disorder, and effective time-reversal symmetry breaking in graphene. Phys. Rev. Lett. 97, 196804 (2006).

[311] X. Wu, X. Li, Z. Song, C. Berger, W. A. de Heer, Weak antilocalization in epitaxial graphene: Evidence for chiral electrons. Phys. Rev. Lett. 98, 136801 (2007).

[312] M. Y. Kharitonov, K. B. Efetov, Universal conductance fluctuations in graphene. Phys. Rev. B 78, 033404 (2008).

[313] D. Klein, Graphitic polymer strips with edge states. Chem. Phys. Lett. 217, 261-265 (1994).

[314] C. H. Lewenkopf, E. R. Mucciolo, A. H. Castro Neto, Numerical studies of conductivity and Fano factor in disordered graphene. Phys. Rev. B 77, 081410 (2008).

[315] E. R. Mucciolo, A. H. Castro Neto, C. H. Lewenkopf, Conductance quantization and transport gaps in disordered graphene nanoribbons. Phys. Rev. B 79, 075407 (2009).

[316] A. Rycerz, Strain-induced transitions to quantum chaos and effective time-reversal symmetry breaking in triangular graphene nanoflakes. Phys. Rev. B 87, 195431 (2013).

[317] B. Dietz, J.-P. Eckmann, C.-A. Pillet, U. Smilansky, I. Ussishkin, Inside-outside duality for planar billiards: A numerical study. Phys. Rev. E 51, 4222-4231 (1995). 
[318] M. Sieber, H. Primack, U. Smilansky, I. Ussishkin, H. Schanz, Semiclassical quantization of billiards with mixed boundary conditions. J. Phys. A Math. Gen. 28, 5041 (1995).

[319] B. Dietz, T. Klaus, M. Miski-Oglu, A. Richter, Spectral properties of superconducting microwave photonic crystals modeling Dirac billiards. Phys. Rev. B 91, 035411 (2015).

[320] P. Šeba, Wave chaos in singular quantum billiard. Phys. Rev. Lett. 64, 1855-1858 (1990).

[321] S. Rahav, O. Richman, S. Fishman, Point perturbations of circle billiards. J. Phys. A Math. Gen. 36, L529 (2003).

[322] F. Leyvraz, C. Schmitd, T. H. Seligman, Anomalous spectral statistics in a symmetrical billiard. $J$. Phys. A Math. Gen. 29, L575-L580 (1996).

[323] T. Fromhold, et al., Chaotic electron diffusion through stochastic webs enhances current flow in superlattices. Nature 428, 726-730 (2004).

[324] M. C. Gutzwiller, Periodic orbits and classical quantization conditions. J. Math. Phys. 12, 343-358 (1971).

[325] D. A. Wisniacki, F. Borondo, E. Vergini, R. M. Benito, Beyond the first recurrence in scar phenomena. Phys. Rev. E 62, R7583-R7586 (2000).

[326] D. Wisniacki, E. Vergini, R. Benito, F. Borondo, Signatures of homoclinic motion in quantum chaos. Phys. Rev. Lett. 94, 054101 (2005).

[327] D. Wisniacki, E. Vergini, R. Benito, F. Borondo, Scarring by homoclinic and heteroclinic orbits. Phys. Rev. Lett. 97, 094101 (2006).

[328] D. Cabosart, et al., Recurrent quantum scars in a mesoscopic graphene ring. Nano Letters $\mathbf{0}$, null (2017).

[329] D. Griffiths, Introduction to Elementary Particles (John Wiley \& Sons, 2008).

[330] L. E. Reichl, I. Prigogine, A Modern Course in Statistical Physics, vol. 71 (University of Texas press Austin, 1980).

[331] J. J. Sakurai, J. Napolitano, Modern quantum mechanics (Addison-Wesley, 2011).

[332] N. Berglund, H. Kunz, Integrability and ergodicity of classical billiards in a magnetic field. J. Stat. Phys. 83, 81-126 (1996).

[333] M. Robnik, Improved statistics of energy levels for Aharonov-Bohm chaotic billiards. J. Phys. A 25, 1399 (1992).

[334] B. Dietz, et al., Induced violation of time-reversal invariance in the regime of weakly overlapping resonances. Phys. Rev. Lett. 103, 064101 (2009).

[335] P. Recher, et al., Aharonov-Bohm effect and broken valley degeneracy in graphene rings. Phys. Rev. $B$ 76, 235404 (2007).

[336] J. Wurm, K. Richter, I. Adagideli, Edge effects in graphene nanostructures: From multiple reflection expansion to density of states. Phys. Rev. B 84, 075468 (2011).

[337] S. C. Creagh, J. M. Robbins, R. G. Littlejohn, Geometrical properties of Maslov indices in the semiclassical trace formula for the density of states. Phys. Rev. A 42, 1907 (1990).

[338] H. Bruus, N. D. Whelan, Edge diffraction, trace formulae and the cardioid billiard. Nonlinearity 9 , 1023 (1996).

[339] S.-Y. Xu, et al., Hedgehog spin texture and Berry's phase tuning in a magnetic topological insulator. Nat. Phys. 8, 616-622 (2012).

[340] A. Kandala, et al., Growth and characterization of hybrid insulating ferromagnet-topological insulator heterostructure devices. Appl. Phys. Lett. 103, 202409 (2013). 
[341] G. J. Ferreira, D. Loss, Magnetically defined qubits on 3D topological insulators. Phys. Rev. Lett. 111, 106802 (2013).

[342] C. F. F. Karney, Long-time correlations in the stochastic regime. Physica D 8, 360-380 (1983).

[343] J. D. Meiss, et al., Correlations of periodic, area-preserving maps. Physica D 6, 375-384 (1983).

[344] J. D. Hanson, J. Cary, J. D. Meiss, Algebraic decay in self-similar Markov chains. J. Stat. Phys. 39, 327-345 (1985).

[345] B. V. Chirikov, D. L. Shepelyansky, Correlation properties of dynamical chaos in Hamiltonian systems. Physica D 13, 395-400 (1984).

[346] J. D. Meiss, E. Ott, Markov-tree model of intrinsic transport in Hamiltonian systems. Phys. Rev. Lett. 55, 2741-2744 (1985).

[347] J. D. Meiss, E. Ott, Markov tree model of transport in area-preserving maps. Physica D 20, 387-402 (1986).

[348] S. Kumar, et al., Distribution of scattering matrix elements in quantum chaotic scattering. Phys. Rev. Lett. 111, 030403 (2013).

[349] E. Doron, U. Smilansky, A. Frenkel, Experimental demonstration of chaotic scattering of microwaves. Phys. Rev. Lett. 65, 3072-3075 (1990).

[350] P. A. Lee, A. D. Stone, Universal conductance fluctuations in metals. Phys. Rev. Lett. 55, 1622-1625 (1985).

[351] C. P. Umbach, S. Washburn, R. B. Laibowitz, R. A. Webb, Magnetoresistance of small, quasi-onedimensional, normal-metal rings and lines. Phys. Rev. B 30, 4048-4051 (1984).

[352] R. A. Webb, S. Washburn, C. P. Umbach, R. B. Laibowitz, Observation of $\frac{h}{e}$ Aharonov-Bohm oscillations in normal-metal rings. Phys. Rev. Lett. 54, 2696-2699 (1985).

[353] A. D. Stone, Magnetoresistance fluctuations in mesoscopic wires and rings. Phys. Rev. Lett. 54, 2692-2695 (1985).

[354] S. Minke, J. Bundesmann, D. Weiss, J. Eroms, Phase coherent transport in graphene nanoribbons and graphene nanoribbon arrays. Phys. Rev. B 86, 155403 (2012).

[355] H. Heersche, P. Jarillo-Herrero, J. Oostinga, L. Vandersypen, A. Morpurgo, Manifestations of phasecoherent transport in graphene. Euro. Phys. J. Spec. Top. 148, 27-37 (2007).

[356] L. A. Ponomarenko, et al., Chaotic Dirac billiard in graphene quantum dots. Science 320, 356-358 (2008).

[357] C. Berger, et al., Electronic confinement and coherence in patterned epitaxial graphene. Science 312, 1191-1196 (2006).

[358] H. B. Heersche, P. Jarillo-Herrero, J. B. Oostinga, L. M. K. Vandersypen, A. F. Morpurgo, Bipolar supercurrent in graphene. Nature 446, 56-59 (2007).

[359] A. Rycerz, J. Tworzydło, C. Beenakker, Anomalously large conductance fluctuations in weakly disordered graphene. Europhys. Lett. 79, 57003 (2007).

[360] B. L. Altshuler, Fluctuations in the extrinsic conductivity of disordered conductors. JETP Lett. 41, 648 (1985)

[361] D. W. Horsell, A. K. Savchenko, F. V. Tikhonenko, K. Kechedzhi, I. V. Lerner, Mesoscopic conductance fluctuations in graphene. Solid State Commun. 149, 1041-1045 (2009).

[362] D. W. Horsell, A. K. Savchenko, F. V. Tikhonenko, K. Kechedzhi, I. V. Lerner, Superconductivityenhanced conductance fluctuations in few-layer graphene. Nanotech. 21, 274005 (2010).

[363] U. Fano, Effects of configuration interaction on intensities and phase shifts. Phys. Rev. 124, 1866$1878(1961)$. 
[364] A. E. Miroshnichenko, S. Flach, Y. S. Kivshar, Fano resonances in nanoscale structures. Rev. Mod. Phys. 82, 2257-2298 (2010).

[365] Y. Yoon, et al., Coupling quantum states through a continuum: A mesoscopic multistate Fano resonance. Phys. Rev. X 2, 021003 (2012).

[366] J. Fransson, et al., Tuning the Fano resonance with an intruder continuum. Nano Lett. 14, 788-793 (2014).

[367] E. J. Heller, Bound-state eigenfunctions of classically chaotic Hamiltonian systems: Scars of periodic orbits. Phys. Rev. Lett. 53, 1515-1518 (1984).

[368] F. J. Arranz, F. Borondo, R. M. Benito, Scar formation at the edge of the chaotic region. Phys. Rev. Lett. 80, 944-947 (1998).

[369] R. Yang, L. Huang, Y.-C. Lai, C. Grebogi, Quantum chaotic scattering in graphene systems. Europhys. Lett. 94, 40004 (2011).

[370] R. P. Taylor, et al., Self-similar magnetoresistance of a semiconductor Sinai billiard. Phys. Rev. Lett. 78, 1952-1955 (1997).

[371] B. Weingartner, S. Rotter, J. Burgdörfer, Simulation of electron transport through a quantum dot with soft walls. Phys. Rev. B 72, 115342 (2005).

[372] M. Mendoza, P. A. Schulz, R. O. Vallejos, C. H. Lewenkopf, Fano resonances in the conductance of quantum dots with mixed dynamics. Phys. Rev. B 77, 155307 (2008).

[373] A. Bäcker, R. Ketzmerick, A. G. Monastra, Flooding of chaotic eigenstates into regular phase space islands. Phys. Rev. Lett. 94, 054102 (2005).

[374] A. Bäcker, et al., Dynamical tunneling in mushroom billiards. Phys. Rev. Lett. 100, 174103 (2008).

[375] S. Löck, A. Bäcker, R. Ketzmerick, P. Schlagheck, Regular-to-chaotic tunneling rates: From the quantum to the semiclassical regime. Phys. Rev. Lett. 104, 114101 (2010).

[376] R. Landauer, Electrical resistance of disordered one-dimensional lattices. Phil. Mag. 21, 863-867 (1970).

[377] S. Datta, Electronic Transport in Mesoscopic systems (Cambridge University Press, 1995).

[378] T. Nakanishi, K. Terakura, T. Ando, Theory of Fano effects in an Aharonov-Bohm ring with a quantum dot. Phys. Rev. B 69, 115307 (2004).

[379] R. Bao, L. Huang, Y.-C. Lai, C. Grebogi, Conductance fluctuations in chaotic bilayer graphene quantum dots. Phys. Rev. E 92, 012918 (2015).

[380] M. Koshino, T. Ando, Transport in bilayer graphene: Calculations within a self-consistent Born approximation. Phys. Rev. B 73, 245403 (2006).

[381] P. R. Wallace, The band theory of graphite. Phys. Rev. 71, 622-634 (1947).

[382] G. Y. Wu, N.-Y. Lue, Y.-C. Chen, Quantum manipulation of valleys in bilayer graphene. Phys. Rev. $B$ 88, 125422 (2013).

[383] E. McCann, M. Koshino, The electronic properties of bilayer graphene. Rep. Prog. Phys. 76, 056503 (2013).

[384] G. A. Luna-Acosta, K. Na, L. E. Reichl, A. Krokhin, Band structure and quantum Poincaré sections of a classically chaotic quantum rippled channel. Phys. Rev. E 53, 3271-3283 (1996).

[385] G. A. Luna-Acosta, A. A. Krokhin, M. A. Rodríguez, P. H. Hernández-Tejeda, Classical chaos and ballistic transport in a mesoscopic channel. Phys. Rev. B 54, 11410-11416 (1996).

[386] W. Breymann, Z. Kovács, T. Tél, Chaotic scattering in the presence of an external magnetic field. Phys. Rev. E 50, 1994-2006 (1994). 
[387] W. John, B. Milek, H. Schanz, P. Šeba, Statistical properties of resonances in quantum irregular scattering. Phys. Rev. Lett. 67, 1949-1952 (1991).

[388] Y. V. Fyodorov, H.-J. Sommers, Statistics of resonance poles, phase shifts and time delays in quantum chaotic scattering: Random matrix approach for systems with broken time-reversal invariance. $J$. Math. Phys. 38, 1918-1981 (1997).

[389] Y. V. Fyodorov, B. A. Khoruzhenko, Systematic analytical approach to correlation functions of resonances in quantum chaotic scattering. Phys. Rev. Lett. 83, 65-68 (1999).

[390] Y. V. Fyodorov, H.-J. Sommers, Random matrices close to Hermitian or unitary: Overview of methods and results. J. Phys. A Math. Gen. 36, 3303-3347 (2003).

[391] A. Ossipov, Y. V. Fyodorov, Statistics of delay times in mesoscopic systems as a manifestation of eigenfunction fluctuations. Phys. Rev. B 71, 125133 (2005).

[392] N. Holmberg, R. Akis, D. P. Pivin, J. P. Bird, D. K. Ferry, Size-dependent effects on the magnetotransport fluctuations of square quantum dots. Semicon. Sci. Tech. 13, A21-A23 (1998).

[393] Y. Ujiie, et al., Regular conductance fluctuations indicative of quasi-ballistic transport in bilayer graphene. J. Phys. Cond. Matt. 21, 382202 (2009).

[394] G. Wang, L. Ying, Y.-C. Lai, Conductance stability in chaotic and integrable quantum dots with random impurities. Phys. Rev. E 92, 022901 (2015).

[395] M. Schneider, P. W. Brouwer, Resonant scattering in graphene with a gate-defined chaotic quantum dot. Phys. Rev. B 84, 115440 (2011).

[396] M. Schneider, P. W. Brouwer, Density of states as a probe of electrostatic confinement in graphene. Phys. Rev. B 89, 205437 (2014).

[397] H. Schomerus, M. Marciani, C. W. J. Beenakker, Effect of chiral symmetry on chaotic scattering from Majorana zero modes. Phys. Rev. Lett. 114, 166803 (2015).

[398] H.-Y. Xu, G.-L. Wang, L. Huang, Y.-C. Lai, Chaos in Dirac electron optics: Emergence of a relativistic quantum chimera. Phys. Rev. Lett. 120, 124101 (2018).

[399] J. Cserti, A. Pályi, C. Péterfalvi, Caustics due to a negative refractive index in circular graphene $p$ - $n$ junctions. Phys. Rev. Lett. 99, 246801 (2007).

[400] V. V. Cheianov, V. Fal'ko, B. L. Altshuler, The focusing of electron flow and a Veselago lens in graphene p-n junctions. Science 315, 1252-1255 (2007).

[401] A. V. Shytov, M. S. Rudner, L. S. Levitov, Klein backscattering and Fabry-Pérot interference in graphene heterojunctions. Phys. Rev. Lett. 101, 156804 (2008).

[402] C. W. J. Beenakker, R. A. Sepkhanov, A. R. Akhmerov, J. Tworzydło, Quantum Goos-Hänchen effect in graphene. Phys. Rev. Lett. 102, 146804 (2009).

[403] A. G. Moghaddam, M. Zareyan, Graphene-based electronic spin lenses. Phys. Rev. Lett. 105, 146803 (2010).

[404] N. Gu, M. Rudner, L. Levitov, Chirality-assisted electronic cloaking of confined states in bilayer graphene. Phys. Rev. Lett. 107, 156603 (2011).

[405] J. R. Williams, T. Low, M. S. Lundstrom, C. M. Marcus, Gate-controlled guiding of electrons in graphene. Nat. Nanotech. 6, 222-225 (2011).

[406] P. Rickhaus, et al., Ballistic interferences in suspended graphene. Nat. Commun. 4, 2342 (2013).

[407] B. Liao, M. Zebarjadi, K. Esfarjani, G. Chen, Isotropic and energy-selective electron cloaks on graphene. Phys. Rev. B 88, 155432 (2013).

[408] R. L. Heinisch, F. X. Bronold, H. Fehske, Mie scattering analog in graphene: Lensing, particle confinement, and depletion of Klein tunneling. Phys. Rev. B 87, 155409 (2013). 
[409] M. M. Asmar, S. E. Ulloa, Rashba spin-orbit interaction and birefringent electron optics in graphene. Phys. Rev. B 87, 075420 (2013).

[410] J.-S. Wu, M. M. Fogler, Scattering of two-dimensional massless Dirac electrons by a circular potential barrier. Phys. Rev. B 90, 235402 (2014).

[411] Y. Zhao, et al., Creating and probing electron whispering-gallery modes in graphene. Science $\mathbf{3 4 8}$, 672-675 (2015).

[412] P. Rickhaus, et al., Guiding of electrons in a few-mode ballistic graphene channel. Nano Lett. 15, 5819-5825 (2015).

[413] G.-H. Lee, G.-H. Park, H.-J. Lee, Observation of negative refraction of Dirac fermions in graphene. Nat. Phys. 11, 925-929 (2015). Letter.

[414] P. Rickhaus, P. Makk, K. Richter, C. Schonenberger, Gate tuneable beamsplitter in ballistic graphene. Appl. Phys. Lett. 107, 251901 (2015).

[415] J. D. Walls, D. Hadad, The Talbot effect for two-dimensional massless Dirac fermions. Sci. Rep. 6, 26698 (2016).

[416] J. Caridad, S. Connaughton, C. Ott, H. B. Weber, V. Krstic, An electrical analogy to Mie scattering. Nat. Commun. 7, 12894 (2016).

[417] C. Gutierrez, L. Brown, C.-J. Kim, J. Park, A. N. Pasupathy, Klein tunnelling and electron trapping in nanometre-scale graphene quantum dots. Nat. Phys. 12, 1069-1075 (2016).

[418] J. Lee, et al., Imaging electrostatically confined Dirac fermions in graphene quantum dots. Nat. Phys. 12, 1032-1036 (2016).

[419] S. Chen, et al., Electron optics with p-n junctions in ballistic graphene. Science 353, 1522-1525 (2016).

[420] M. Settnes, S. R. Power, M. Brandbyge, A.-P. Jauho, Graphene nanobubbles as valley filters and beam splitters. Phys. Rev. Lett. 117, 276801 (2016).

[421] M.-H. Liu, C. Gorini, K. Richter, Creating and steering highly directional electron beams in graphene. Phys. Rev. Lett. 118, 066801 (2017).

[422] A. W. Barnard, et al., Absorptive pinhole collimators for ballistic Dirac fermions in graphene. Nat. Commun. 8, 15418 (2017).

[423] Y. Jiang, et al., Tuning a circular p-n junction in graphene from quantum confinement to optical guiding. Nat. Nanotech. 12, 1045-1049 (2017).

[424] F. Ghahari, et al., An on/off Berry phase switch in circular graphene resonators. Science 356, 845849 (2017).

[425] S.-H. Zhang, J.-J. Zhu, W. Yang, K. Chang, Focusing RKKY interaction by graphene p-n junction. 2D Mater. 4, 035005 (2017).

[426] P. Bãggild, et al., A two-dimensional Dirac fermion microscope. Nat. Commun. 8, 15783 (2017).

[427] Y. Kuramoto, D. Battogtokh, Coexistence of coherence and incoherence in nonlocally coupled phase oscillators. Nonlinear Phenom. Complex Syst. 5, 380-385 (2002).

[428] D. M. Abrams, S. H. Strogatz, Chimera states for coupled oscillators. Phys. Rev. Lett. 93, 174102 (2004).

[429] D. M. Abrams, S. H. Strogatz, Chimera states in a ring of nonlocally coupled oscillators. Int. J. Bif. Chaos 16, 21-37 (2006).

[430] M. R. Tinsley, S. Nkomo, K. Showalter, Chimera and phase-cluster states in populations of coupled chemical oscillators. Nat. Phys. 8, 662 (2012). 
[431] A. M. Hagerstrom, et al., Experimental observation of chimeras in coupled-map lattices. Nat. Phys. 8, 658 (2012).

[432] E. A. Martens, S. Thutupalli, A. Fourrière, O. Hallatschek, Chimera states in mechanical oscillator networks. Proc. Nat. Acad. Sci. (USA) 110, 10563-10567 (2013).

[433] N. Yao, Z.-G. Huang, Y.-C. Lai, Z.-G. Zheng, Robustness of chimera states in complex dynamical systems. Sci. Rep. 3, 3522 (2013).

[434] N. Yao, Z.-G. Huang, C. Grebogi, Y.-C. Lai, Emergence of multicluster chimera states. Sci. Rep. 5, 12988 (2015).

[435] L. A. Bunimovich., Mushrooms and other billiards with divided phase space. Chaos 11, 802-808 (2001).

[436] A. M. Burke, et al., Periodic scarred states in open quantum dots as evidence of quantum Darwinism. Phys. Rev. Lett. 104, 176801 (2010).

[437] E. Ott, Chaos in Dynamical Systems (Cambridge University Press, Cambridge UK, 2002), second edn.

[438] I. V. Fialkovsky, D. V. Vassilevich, Quantum field theory in graphene. Int. J. Mod. Phys. Conf. Ser. 14, 88-99 (2012).

[439] V. Gusynin, S. Sharapov, J. Carbotte, AC conductivity of graphene: From tight-binding model to 2+1-dimensional quantum electrodynamics. Int. J. Mod. Phys. B 21, 4611-4658 (2007).

[440] C. Beneventano, E. Santangelo, Boundary conditions in the Dirac approach to graphene devices. Int. J. Mod. Phys. Conf. Ser. 14, 240-249 (2012).

[441] J. Bolte, S. Keppeler, Semiclassical time evolution and trace formula for relativistic spin-1/2 particles. Phys. Rev. Lett. 81, 1987-1991 (1998).

[442] J. Bolte, S. Keppeler, A semiclassical approach to the Dirac equation. Ann. Phys. (N.Y.) 274, 125-162 (1999).

[443] J. Bolte, S. Keppeler, Semiclassical form factor for chaotic systems with spin 1/2. J. Phys. A Math. Gen. 32, 8863 (1999).

[444] J. Bolte, J. Harrison, Spectral statistics for the Dirac operator on graphs. J. Phys. A Math. Gen. 36, 2747 (2003).

[445] M. V. Berry, Regular and irregular semiclassical wavefunctions. J. Phys. A Math. Gen. 10, 2083 (1977).

[446] C. Zhang, J. Liu, M. G. Raizen, Q. Niu, Quantum chaos of Bogoliubov waves for a Bose-Einstein condensate in stadium billiards. Phys. Rev. Lett. 93, 074101 (2004).

[447] M. Akila, D. Waltner, B. Gutkin, P. Braun, T. Guhr, Semiclassical identification of periodic orbits in a quantum many-body system. Phys. Rev. Lett. 118, 164101 (2017).

[448] F. H. L. Essler, The One-Dimensional Hubbard Model (Cambridge University Press, Cambridge UK, 2005).

[449] A. Altland, B. Simons, Condensed Matter Field Theory (Cambridge University Press, New York, 2010), second edn.

[450] T. Ando, Theory of electronic states and transport in carbon nanotubes. J. Phys. Soc. Jpn. 74, 777-817 (2005).

[451] J. Martin, et al., Observation of electron-hole puddles in graphene using a scanning single-electron transistor. Nat. Phys. 4, 144-148 (2008).

[452] C. L. Kane, M. P. A. Fisher, Transmission through barriers and resonant tunneling in an interacting one-dimensional electron gas. Phys. Rev. B 46, 15233-15262 (1992). 
[453] M. Golor, S. Wessel, M. J. Schmidt, Quantum nature of edge magnetism in graphene. Phys. Rev. Lett. 112, 046601 (2014).

[454] M. Drienovsky, et al., Towards superlattices: Lateral bipolar multibarriers in graphene. Phys. Rev. B 89, 115421 (2014).

[455] H.-F. Cheung, E. K. Riedel, Y. Gefen, Persistent currents in mesoscopic rings and cylinders. Phys. Rev. Lett. 62, 587-590 (1989).

[456] A. Schmid, Persistent currents in mesoscopic rings by suppression of charge fluctuations. Phys. Rev. Lett. 66, 80-83 (1991).

[457] H. Bouchiat, New clues in the mystery of persistent currents. Phys. 1, 7 (2008).

[458] U. Sivan, Y. Imry, de Haas-van Alphen and Aharonov-Bohm-type persistent current oscillations in singly connected quantum dots. Phys. Rev. Lett. 61, 1001-1004 (1988).

[459] F. von Oppen, E. K. Riedel, Average persistent current in a mesoscopic ring. Phys. Rev. Lett. 66, 84-87 (1991).

[460] V. Ambegaokar, U. Eckern, Coherence and persistent currents in mesoscopic rings. Phys. Rev. Lett. 65, 381-384 (1990).

[461] R. Berkovits, Y. Avishai, Interacting electrons in disordered potentials: Conductance versus persistent currents. Phys. Rev. Lett. 76, 291-294 (1996).

[462] J. Splettstoesser, M. Governale, U. Zülicke, Persistent current in ballistic mesoscopic rings with Rashba spin-orbit coupling. Phys. Rev. B 68, 165341 (2003).

[463] J. S. Sheng, K. Chang, Spin states and persistent currents in mesoscopic rings: Spin-orbit interactions. Phys. Rev. B 74, 235315 (2006).

[464] V. Gudmundsson, C.-S. Tang, A. Manolescu, Nonadiabatic current generation in a finite width semiconductor ring. Phys. Rev. B 67, 161301 (2003).

[465] A. Matos-Abiague, J. Berakdar, Photoinduced charge currents in mesoscopic rings. Phys. Rev. Lett. 94, 166801 (2005).

[466] H.-F. Cheung, Y. Gefen, E. K. Riedel, W.-H. Shih, Persistent currents in small one-dimensional metal rings. Phys. Rev. B 37, 6050-6062 (1988).

[467] F. von Oppen, E. K. Riedel, Average persistent current in a mesoscopic ring. Phys. Rev. Lett. 66, 84-87 (1991).

[468] J. F. Weisz, R. Kishore, F. V. Kusmartsev, Persistent current in isolated mesoscopic rings. Phys. Rev. B 49, 8126-8131 (1994).

[469] A. Bruno-Alfonso, A. Latgé, Quantum rings of arbitrary shape and non-uniform width in a threading magnetic field. Phys. Rev. B 77, 205303 (2008).

[470] H. Bary-Soroker, O. Entin-Wohlman, Y. Imry, Persistent currents of noninteracting electrons in one-, two-, and three-dimensional thin rings. Phys. Rev. B 82, 144202 (2010).

[471] T. Ihn, A. Fuhrer, L. Meier, M. Sigrist, K. Ensslin, Quantum physics in quantum rings. Europhys. News 36, 78-81 (2005).

[472] F. von Oppen, E. K. Riedel, Quantum persistent currents and classical periodic orbits. Phys. Rev. B 48, 9170-9173 (1993).

[473] S. Kawabata, Persistent currents in quantum chaotic systems. Phys. Rev. B 59, 12256-12259 (1999).

[474] A. H. Castro Neto, F. Guinea, N. M. R. Peres, Edge and surface states in the quantum Hall effect in graphene. Phys. Rev. B 73, 205408 (2006).

[475] D. S. L. Abergel, V. M. Apalkov, T. Chakraborty, Interplay between valley polarization and electronelectron interaction in a graphene ring. Phys. Rev. B 78, 193405 (2008). 
[476] C. W. J. Beenakker, A. R. Akhmerov, P. Recher, J. Tworzydło, Correspondence between Andreev reflection and Klein tunneling in bipolar graphene. Phys. Rev. B 77, 075409 (2008).

[477] M. Zarenia, J. M. Pereira, F. M. Peeters, G. A. Farias, Electrostatically confined quantum rings in bilayer graphene. Nano Lett. 9, 4088-4092 (2009).

[478] R. Jackiw, A. I. Milstein, S.-Y. Pi, I. S. Terekhov, Induced current and Aharonov-Bohm effect in graphene. Phys. Rev. B 80, 033413 (2009).

[479] M. M. Ma, J. W. Ding, N. Xu, Odd-even width effect on persistent current in zigzag hexagonal graphene rings. Nanoscale 1, 387-390 (2009).

[480] M. Zarenia, J. M. Pereira, A. Chaves, F. M. Peeters, G. A. Farias, Simplified model for the energy levels of quantum rings in single layer and bilayer graphene. Phys. Rev. B 81, 045431 (2010).

[481] D. Soriano, J. Fernández-Rossier, Spontaneous persistent currents in a quantum spin Hall insulator. Phys. Rev. B 82, 161302 (2010).

[482] P. Michetti, P. Recher, Bound states and persistent currents in topological insulator rings. Phys. Rev. $B$ 83, 125420 (2011).

[483] B.-L. Huang, M.-C. Chang, C.-Y. Mou, Persistent currents in a graphene ring with armchair edges. J. Phys. Condens. Mat. 24, 245304 (2012).

[484] D. Faria, A. Latgé, S. E. Ulloa, N. Sandler, Currents and pseudomagnetic fields in strained graphene rings. Phys. Rev. B 87, 241403 (2013).

[485] D. Sticlet, B. Dóra, J. Cayssol, Persistent currents in Dirac fermion rings. Phys. Rev. B 88, 205401 (2013).

[486] N. Bolívar, E. Medina, B. Berche, Persistent charge and spin currents in the long-wavelength regime for graphene rings. Phys. Rev. B 89, 125413 (2014).

[487] J. U. Nöckel, A. D. Stone, G. Chen, H. L. Grossman, R. K. Chang, Directional emission from asymmetric resonant cavities. Opt. Lett. 21, 1609-1611 (1996).

[488] C. Gmachl, et al., High-power directional emission from microlasers with chaotic resonators. Science 280, 1556-1564 (1998).

[489] S. M. Spillane, T. J. Kippenberg, K. J. Vahala, Ultralow-threshold Raman laser using a spherical dielectric microcavity. Nature 415, 621-623 (2002).

[490] K. J. Vahala, Optical microcavities. Nature 424, 839-846 (2003).

[491] Z. Z. Zhang, K. Chang, F. M. Peeters, Tuning of energy levels and optical properties of graphene quantum dots. Phys. Rev. B 77, 235411 (2008).

[492] K. Chang, W.-K. Lou, Helical quantum states in HgTe quantum dots with inverted band structures. Phys. Rev. Lett. 106, 206802 (2011).

[493] G. Reecht, et al., Oligothiophene nanorings as electron resonators for whispering gallery modes. Phys. Rev. Lett. 110, 056802 (2013).

[494] S. Schnez, K. Ensslin, M. Sigrist, T. Ihn, Analytic model of the energy spectrum of a graphene quantum dot in a perpendicular magnetic field. Phys. Rev. B 78 (2008).

[495] A. N. Redlich, Parity violation and gauge noninvariance of the effective gauge field action in three dimensions. Phys. Rev. D 29, 2366-2374 (1984).

[496] T. Appelquist, M. J. Bowick, D. Karabali, L. C. R. Wijewardhana, Spontaneous breaking of parity in (2+1)-dimensional QED. Phys. Rev. D 33, 3774-3776 (1986).

[497] A. Hosaka, H. Toki, Chiral bag model for the nucleon. Phys. Rep. 277, 65-188 (1996).

[498] G. Esposito, K. Kirsten, Chiral bag boundary conditions on the ball. Phys. Rev. D 66, 085014 (2002). 
[499] F. S. Nogueira, I. Eremin, Thermal screening at finite chemical potential on a topological surface and its interplay with proximity-induced ferromagnetism. Phys. Rev. B 90 (2014).

[500] T. Hayashi, T. Fujisawa, H. D. Cheong, Y. H. Jeong, Y. Hirayama, Coherent manipulation of electronic states in a double quantum dot. Phys. Rev. Lett. 91, 226804 (2003).

[501] S. N. Shevchenko, S. Ashhab, F. Nori, Landau-Zener-Stückelberg interferometry. Phys. Rep. 492 , 1-30 (2010).

[502] E. K. Riedel, F. von Oppen, Mesoscopic persistent current in small rings. Phys. Rev. B 47, 1544915459 (1993).

[503] H. Zhang, et al., Topological insulators in $\mathrm{Bi}_{2} \mathrm{Se}_{3}, \mathrm{Bi}_{2} \mathrm{Te}_{3}$ and $\mathrm{Sb}_{2} \mathrm{Te}_{3}$ with a single Dirac cone on the surface. Nat. Phys. 5, 438-442 (2009).

[504] Y. Xia, et al., Observation of a large-gap topological-insulator class with a single Dirac cone on the surface. Nat. Phys. 5, 398-402 (2009).

[505] H. Beidenkopf, et al., Spatial fluctuations of helical Dirac fermions on the surface of topological insulators. Nat. Phys. 7, 939-943 (2011).

[506] W. Rabaud, et al., Persistent currents in mesoscopic connected rings. Phys. Rev. Lett. 86, 3124-3127 (2001).

[507] N. A. J. M. Kleemans, et al., Oscillatory persistent currents in self-assembled quantum rings. Phys. Rev. Lett. 99, 146808 (2007).

[508] J. D. Barrow, General relativistic chaos and nonlinear dynamics. Gen. Rela. Grav. 14, 523-530 (1982).

[509] S. P. Drake, C. P. Dettmann, N. E. Frankel, N. J. Cornish, Chaos in special relativistic dynamics. Phys. Rev. E 53, 1351-1361 (1996).

[510] J. V. Podolský, K. Veselý, Chaos in pp-wave spacetimes. Phys. Rev. D 58, 081501 (1998).

[511] J. D. Barrow, J. Levin, Chaos in the Einstein-Yang-Mills equations. Phys. Rev. Lett. 80, 656-659 (1998).

[512] R. Tomaschitz, Tachyons, Lamb shifts and superluminal chaos. Euro. Phys. J. B Cond. Mat. Comp. Sys. 17, 523-536 (2000).

[513] T. Kovacs, G. Bene, T. Tel, Relativistic effects in the chaotic Sitnikov problem. Mon. Not. R. Astron. Soc. 414, 2275-2281 (2011).

[514] J. D. Bernal, J. M. Seoane, M. A. F. Sanjuán, Global relativistic effects in chaotic scattering. Phys. Rev. E 95, 032205 (2017).

[515] H. Primack, U. Smilansky, On the accuracy of the semiclassical trace formula. J. Phys. A. Math. Gen. 31, 6253-6277 (1998).

[516] D. Cohen, H. Primack, U. Smilansky, Quantal-classical duality and the semiclassical trace formula. Ann. Phys. 264, 108-170 (1998).

[517] U. Smilansky, Trace identities and their semiclassical implications. J. Phys. A. Math. Gen. 33, 2299$2312(2000)$.

[518] H. Primack, U. Smilansky, The quantum three-dimensional Sinai billiard - a semiclassical analysis. Phys. Rep. 327, 1-107 (2000).

[519] P. Carmier, D. Ullmo, Berry phase in graphene: Semiclassical perspective. Phys. Rev. B 77, 245413 (2008).

[520] J. Wurm, K. Richter, I. Adagideli, Edge effects in graphene nanostructures: From multiple reflection expansion to density of states. Phys. Rev. B 84, 075468 (2011). 
[521] H.-Y. Xu, Y.-C. Lai, Revival resonant scattering, perfect caustics, and isotropic transport of pseudospin-1 particles. Phys. Rev. B 94, 165405 (2016).

[522] E. I. Rashba, Properties of semiconductors with an extremum loop. 1. cyclotron and combinational resonance in a magnetic field perpendicular to the plane of the loop. Sov. Phys. Solid State 2, 1109$1122(1960)$.

[523] G. Dresselhaus, M. S. Dresselhaus, Spin-orbit interaction in graphite. Phys. Rev. 140, A401-A412 (1965).

[524] K. Taguchi, K. Shintani, Y. Tanaka, Spin-charge transport driven by magnetization dynamics on the disordered surface of doped topological insulators. Phys. Rev. B 92, 035425 (2015).

[525] G.-L. Wang, H.-Y. Xu, Y.-C. Lai, Nonlinear dynamics induced anomalous Hall effect in topological insulators. Sci. Rep. 6, 19803 (2016).

[526] B. A. Bernevig, T. L. Hughes, S.-C. Zhang, Quantum spin Hall effect and topological phase transition in HgTe quantum wells. Science 314, 1757-1761 (2006).

[527] J. C. Slonczewski, Current-driven excitation of magnetic multilayers. J. Mag. Mag. Mate. 159, L1L7 (1996).

[528] A. Mellnik, et al., Spin-transfer torque generated by a topological insulator. Nature 511, 449-451 (2014).

[529] D. C. Ralph, M. D. Stiles, Spin transfer torques. J. Magnetism Mag. Mater. 320, 1190-1126 (2008).

[530] M. Goerbig, G. Montambaux, Dirac Fermions in Condensed Matter and Beyond (Springer International Publishing, Cham, 2017), pp. 25-53.

[531] C. Beenakker, Search for Majorana fermions in superconductors. Annu. Rev. Condens. Mat. Phys. 4, 113-136 (2013).

[532] N. P. Armitage, E. J. Mele, A. Vishwanath, Weyl and dirac semimetals in three-dimensional solids. Rev. Mod. Phys. 90, 015001 (2018).

[533] X. Huang, Y. Lai, Z. H. Hang, H. Zheng, C. T. Chan, Dirac cones induced by accidental degeneracy in photonic crystals and zero-refractive-index materials. Nat. Mater 10, 582-586 (2011).

[534] J. Mei, Y. Wu, C. T. Chan, Z.-Q. Zhang, First-principles study of Dirac and Dirac-like cones in phononic and photonic crystals. Phys. Rev. B 86, 035141 (2012).

[535] P. Moitra, et al., Realization of an all-dielectric zero-index optical metamaterial. Nat. Photon. 7, $791-795$ (2013).

[536] Y. Li, et al., On-chip zero-index metamaterials. Nat. Photon. 9, 738-742 (2015).

[537] A. Fang, Z. Q. Zhang, S. G. Louie, C. T. Chan, Klein tunneling and supercollimation of pseudospin-1 electromagnetic waves. Phys. Rev. B 93, 035422 (2016).

[538] D. Guzmán-Silva, et al., Experimental observation of bulk and edge transport in photonic Lieb lattices. New J. Phys. 16, 063061 (2014).

[539] S. Mukherjee, et al., Observation of a localized flat-band state in a photonic Lieb lattice. Phys. Rev. Lett. 114, 245504 (2015).

[540] R. A. Vicencio, et al., Observation of localized states in Lieb photonic lattices. Phys. Rev. Lett. 114, 245503 (2015).

[541] F. Diebel, D. Leykam, S. Kroesen, C. Denz, A. S. Desyatnikov, Conical diffraction and composite Lieb bosons in photonic lattices. Phys. Rev. Lett. 116, 183902 (2016).

[542] S. Taie, et al., Coherent driving and freezing of bosonic matter wave in an optical Lieb lattice. Sci. $A d v .1$ (2015). 
[543] D. Bercioux, D. F. Urban, H. Grabert, W. Häusler, Massless Dirac-Weyl fermions in a $T_{3}$ optical lattice. Phys. Rev. A 80, 063603 (2009).

[544] H. Xu, Y.-C. Lai, Superscattering of a pseudospin-1 wave in a photonic lattice. Phys. Rev. A 95, 012119 (2017).

[545] M. Yamamoto, Y. Shimazaki, I. V. Borzenets, S. Tarucha, Valley Hall effect in two-dimensional hexagonal lattices. J. Phys. Soc. Jpn. 84, 121006 (2015).

[546] X. Xu, W. Yao, D. Xiao, T. F. Heinz, Spin and pseudospins in layered transition metal dichalcogenides. Nat. Phys. 10, 343-350 (2014).

[547] J. L. Garcia-Pomar, A. Cortijo, M. Nieto-Vesperinas, Fully valley-polarized electron beams in graphene. Phys. Rev. Lett. 100, 236801 (2008).

[548] J. Lu, C. Qiu, M. Ke, Z. Liu, Valley vortex states in sonic crystals. Phys. Rev. Lett. 116, 093901 (2016).

[549] J.-W. Dong, X.-D. Chen, H. Zhu, Y. Wang, X. Zhang, Valley photonic crystals for control of spin and topology. Nat. Mater. 16, 298-302 (2017).

[550] J. R. Schaibley, et al., Valleytronics in 2D materials. Nat. Rev. Mater. 1, 16055 (2016).

[551] K. Behnia, Polarized light boosts valleytronics. Nat. Nanotech. 7, 488-489 (2012).

[552] C. E. Nebel, Electron dance in diamond. Nat. Mater. 12, 690-692 (2013).

[553] R. V. Gorbachev, et al., Detecting topological currents in graphene superlattices. Science 346, 448451 (2014).

[554] A. Kundu, H. A. Fertig, B. Seradjeh, Floquet-engineered valleytronics in Dirac systems. Phys. Rev. Lett. 116, 016802 (2016).

[555] Y. Ye, et al., Electrical generation and control of valley carriers in a monolayer transition metal dichalcogenide. Nat. Nanotech. 11, 598-602 (2016).

[556] V. H. Nguyen, S. Dechamps, P. Dollfus, J.-C. Charlier, Valley filtering and electronic optics using polycrystalline graphene. Phys. Rev. Lett. 117, 247702 (2016).

[557] M. Trushin, J. Schliemann, Pseudospin in optical and transport properties of graphene. Phys. Rev. Lett. 107, 156801 (2011).

[558] D. Xiao, G.-B. Liu, W. Feng, X. Xu, W. Yao, Coupled spin and valley physics in monolayers of $\mathrm{MoS}_{2}$ and other group-VI dichalcogenides. Phys. Rev. Lett. 108, 196802 (2012).

[559] Y. D. Lensky, J. C. W. Song, P. Samutpraphoot, L. S. Levitov, Topological valley currents in gapped Dirac materials. Phys. Rev. Lett. 114, 256601 (2015).

[560] J. C. W. Song, P. Samutpraphoot, L. S. Levitov, Topological Bloch bands in graphene superlattices. Proc. Nat. Acad. Sci. (USA) 112, 10879-10883 (2015).

[561] K. F. Mak, K. L. McGill, J. Park, P. L. McEuen, The valley Hall effect in $\mathrm{MoS}_{2}$ transistors. Science 344, 1489-1492 (2014).

[562] M. Sui, et al., Gate-tunable topological valley transport in bilayer graphene. Nat. Phys. 11, 10271031 (2015).

[563] Y. Shimazaki, et al., Generation and detection of pure valley current by electrically induced Berry curvature in bilayer graphene. Nat. Phys. 11, 1032-1036 (2015).

[564] J. Lee, K. F. Mak, J. Shan, Electrical control of the valley Hall effect in bilayer $\mathrm{MoS}_{2}$ transistors. Nat. Nanotech. 11, 421-425 (2016).

[565] A. Chaves, L. Covaci, K. Y. Rakhimov, G. A. Farias, F. M. Peeters, Wave-packet dynamics and valley filter in strained graphene. Phys. Rev. B 82, 205430 (2010). 
[566] Y. Jiang, T. Low, K. Chang, M. I. Katsnelson, F. Guinea, Generation of pure bulk valley current in graphene. Phys. Rev. Lett. 110, 046601 (2013).

[567] M. M. Grujić, M. Z. Tadić, F. Peeters, Spin-valley filtering in strained graphene structures with artificially induced carrier mass and spin-orbit coupling. Phys. Rev. Lett. 113, 046601 (2014).

[568] H.-Y. Xu, L. Huang, D. Huang, Y.-C. Lai, Geometric valley Hall effect and valley filtering through a singular Berry flux. Phys. Rev. B 96, 045412 (2017).

[569] G. W. Semenoff, Condensed-matter simulation of a three-dimensional anomaly. Phys. Rev. Lett. 53, 2449-2452 (1984).

[570] F. D. M. Haldane, Model for a quantum Hall effect without Landau levels: Condensed-matter realization of the "parity anomaly". Phys. Rev. Lett. 61, 2015-2018 (1988).

[571] R. E. Wagner, P. J. Peverly, Q. Su, R. Grobe, Classical versus quantum dynamics for a driven relativistic oscillator. Phys. Rev. A 61, 035402 (2000).

[572] G. Casati, B. Chirikov, Quantum Chaos: Between Order and Disorder (Cambridge University Press, 2006).

[573] D. U. Matrasulov, G. M. Milibaeva, U. R. Salomov, B. Sundaram, Relativistic kicked rotor. Phys. Rev. E 72, 016213 (2005).

[574] Q. Zhao, C. A. Müller, J. Gong, Quantum and classical superballistic transport in a relativistic kickedrotor system. Phys. Rev. E 90, 022921 (2014).

[575] E. B. Rozenbaum, V. Galitski, Dynamical localization of coupled relativistic kicked rotors. Phys. Rev. B 95, 064303 (2017). 